\title{
Translational control during hypoxia: consequences for gene expression and hypoxia tolerance
}

Citation for published version (APA):

van den Beucken, A. M. (2008). Translational control during hypoxia: consequences for gene expression and hypoxia tolerance. [Doctoral Thesis, Maastricht University]. Universiteit Maastricht. https://doi.org/10.26481/dis.20080911ab

Document status and date:

Published: 01/01/2008

DOI:

10.26481/dis.20080911ab

Document Version:

Publisher's PDF, also known as Version of record

\section{Please check the document version of this publication:}

- A submitted manuscript is the version of the article upon submission and before peer-review. There can be important differences between the submitted version and the official published version of record.

People interested in the research are advised to contact the author for the final version of the publication, or visit the DOI to the publisher's website.

- The final author version and the galley proof are versions of the publication after peer review.

- The final published version features the final layout of the paper including the volume, issue and page numbers.

Link to publication

\footnotetext{
General rights rights.

- You may freely distribute the URL identifying the publication in the public portal. please follow below link for the End User Agreement:

www.umlib.nl/taverne-license

Take down policy

If you believe that this document breaches copyright please contact us at:

repository@maastrichtuniversity.nl

providing details and we will investigate your claim.
}

Copyright and moral rights for the publications made accessible in the public portal are retained by the authors and/or other copyright owners and it is a condition of accessing publications that users recognise and abide by the legal requirements associated with these

- Users may download and print one copy of any publication from the public portal for the purpose of private study or research.

- You may not further distribute the material or use it for any profit-making activity or commercial gain

If the publication is distributed under the terms of Article $25 \mathrm{fa}$ of the Dutch Copyright Act, indicated by the "Taverne" license above, 


\title{
Translational control during hypoxia:
}

\author{
consequences for gene expression \\ and hypoxia tolerance
}


Printed by: Datawyse| Universitaire Pers Maastricht ISBN: 978-90-9023370-3

(C) Copyright Twan van den Beucken, Maastricht 2008 


\title{
Translational control during hypoxia:
}

\author{
consequences for gene expression \\ and hypoxia tolerance
}

\author{
PROEFSCHRIFT \\ ter verkrijging van de graad van doctor aan de Universiteit Maastricht, \\ op gezag van de Rector Magnificus, Prof. mr. G.P.M.F. Mols \\ volgens het besluit van het College van Decanen, \\ in het openbaar te verdedigen op \\ donderdag 11 september 2008 om 12:00 uur \\ door
}

Antonius Marie van den Beucken 
PROMOTORES:

Prof. dr. B.G. Wouters

Prof. dr. P. Lambin

COPROMOTOR:

Dr. M. Koritzinsky

BEOORDELINGSCOMMISSIE:

Prof. dr. A.W. Griffioen (Voorzitter)

Prof. dr. A.C. Begg (NKI, Amsterdam)

Prof. dr. A.P. de Bruïne

Prof. dr. F.C.S. Ramaekers

Dr. H.J.M. Smeets

Financial support for this thesis publication was provided by Becton Dickinson Benelux N.V. 


\section{Contents}

Chapter $1 \quad$ General introduction 7

Chapter $2 \quad$ Gene expression during acute and prolonged 39 hypoxia is regulated by distinct mechanisms of translational control

Chapter 3 Translational control of gene expression during hypoxia

Chapter 4 The hypoxic proteome is influenced by gene-specific changes in mRNA translation

Chapter 5 Regulation of mRNA translation is a major

Chapter $6 \quad$ Regulation of Cited 2 expression provides a functional link between translational and transcriptional responses during hypoxia

Chapter $7 \quad$ Hypoxia-induced carbonic anhydrase 9 expression is dependent on the unfolded protein response

Chapter 8 Targeting translational control pathways; an in vitro study

Chapter 9 General discussion and summary

Algemene discussie en samenvatting 


\section{List of abbreviations}

\begin{tabular}{|c|c|}
\hline AMP & adenosine monophosphate \\
\hline AMPK & amp activated protein kinase \\
\hline ARNT & aryl hydrocarbon receptor nuclear translocator \\
\hline ATF & activating transcription factor \\
\hline ATP & adenosine triphosphate \\
\hline $\mathrm{BiP}$ & immunoglobulin binding protein \\
\hline $\mathrm{BP}$ & binding protein \\
\hline CAIX & carbonic anhydrase IX \\
\hline $\mathrm{CHOP}$ & C/EBP homologous protein \\
\hline CITED & $\begin{array}{l}\text { CREB-binding protein/p300-interacting transactivator with } \\
\text { ED- rich tail }\end{array}$ \\
\hline DDIT & dna damage inducible transcript \\
\hline DNA & deoxyribonucleic acid \\
\hline eIF & eukaryotic initiation factor \\
\hline E.R. & Endoplasmic reticulum \\
\hline GADD & growth arrest \\
\hline GTP & guanidine triphosphate \\
\hline HIF & hypoxia inducible factor \\
\hline HK & hexokinase \\
\hline HRE & hypoxia responsive element \\
\hline HSP & heat shock protein \\
\hline IRE & inositol requiring protein \\
\hline ISR & integrated stress response \\
\hline $\mathrm{kDa}$ & kilo Dalton \\
\hline MEF & mouse embryonic fibroblast \\
\hline mRNA & messenger RNA \\
\hline mTOR & mammalian target of rapamycin \\
\hline PBS & phosphate buffered saline \\
\hline PCR & polymerase chain reaction \\
\hline PDK & phosphatidylinositol dependent kinase \\
\hline PHD & prolyl hydroxylase domain \\
\hline PI3K & phosphatidylinositol 3 kinase \\
\hline PJS & Peutz-Jeghers syndrome \\
\hline PKR & protein kinase RNA \\
\hline PTEN & phosphatase and tensin homologue \\
\hline RNA & ribonucleic acid \\
\hline S51A & serine 51 to alanine mutation \\
\hline SD & standard deviation \\
\hline SEM & standard error of the mean \\
\hline TSC & tuberous sclerosis complex \\
\hline Q-PCR & quantitative PCR \\
\hline UPR & unfolded protein response \\
\hline VEGF & vascular endothelial growth factor \\
\hline VHL & Von-Hippel Lindau \\
\hline W.T. & wild-type \\
\hline
\end{tabular}




\section{CHAPTER 1}

\section{General introduction}

Adapted from:

Wouters BG, van den Beucken T, Magagnin MG, Koritzinsky M, Fels D, Koumenis C. Semin Cell Dev Biol. 2005 AugOct; 16(4-5):487-501.

Wouters BG, van den Beucken T, Magagnin MG, Lambin P, Koumenis C. Drug Resist Updat. 2004 Feb;7(1):25-40. 


\section{Cancer and hypoxia}

Cancer remains a highly prevalent disease that has a tremendous cost to afflicted individuals, their families, and to society in general. In fact, 7.6 million people died word wide in 2007 alone according to the American Cancer Society ${ }^{1}$. The term "cancer" refers to a collection of related diseases in which normal cells in the body acquire a series of genetic changes that enable them to proliferate in an uncontrolled manner. This results in the formation of a tumor mass or neoplasm at the site from which the cancer cell arose. The cancer cells often acquire further genetic changes that enable them to invade nearby tissues and to spread via the bloodstream or lymphatic system to other tissues in the body, a process known as metastasis.

The goal of current cancer treatment is to offer affected patients an improved quality of life by achieving cure of the disease, increasing their lifespan, or by diminishing symptomatic side effects. The standard treatment options available for cancer include surgery, chemotherapy and radiotherapy. For certain tumor types additional therapies such as hormone therapy (breast, prostate) or immunotherapy (melanoma) can be given. Advances in cancer research have certainly led to better prognosis for many cancer types especially in those cases where early diagnosis of the disease can be achieved. Improved methods for administering standard therapies, alone or in combination have also improved cancer therapy. For example, new technologies allow for much more conformed radiation dose delivery, allowing for reduced normal tissue toxicity and increased dose to the tumor. Nonetheless, available therapy remains ineffective in a large fraction of patients for reasons that are not clear. One of the potential explanations for the large degree of variability in patient response to treatment is the tremendous heterogeneity of the tumor microenvironment. The microenvironment of human tumors is unlike that of any other normal tissue, characterized by heterogeneities in nutrient supply, $\mathrm{pH}$, and oxygenation. These unique features develop as a consequence of alterations in the metabolic and 
proliferative status of the tumor cells together with a highly irregular vascular supply. In addition to the genetic and epigenetic changes that occur in cancer, this microenvironment is recognized to be an important contributor to altered gene expression and cellular function in cancer. In particular, both laboratory and clinical evidence strongly associate deficiencies in oxygenation (hypoxia) with tumor development, growth, metastasis and poor response to therapy. Hypoxia is known to directly influence the efficacy of cancer treatment (radiotherapy and chemotherapy) and also alters the behavior of tumor cells through a number of oxygen sensitive signaling pathways. This realization has stimulated efforts to understand the mechanisms through which hypoxia influences cell and cancer biology, and to develop new approaches to therapy by targeting these biological responses.

\subsection{Hypoxia, an intrinsic resistance to therapy}

The potential contribution of hypoxia to treatment resistance has been recognized for a long time. For radiotherapy, the lack of oxygen alters the chemical reactions which take place in the DNA upon irradiation such that only about one third as many lethal DNA lesions are produced in hypoxic cells compared with aerobic cells ${ }^{2}$. For systemically delivered therapies, the hypoxic areas in tumors have also been thought to provide foci of resistance $e^{3-5}$.This is due in part to the unusual, chaotic, and insufficient organization of blood vessels within tumors which impairs the ability to deliver both oxygen and treatment to isolated regions of tumors ${ }^{4,6,7}$. The poorly vascularized regions also limit cell proliferation which can provide resistance towards many chemotherapeutic agents targeted at proliferating cells. The intrinsic resistance to therapy caused by hypoxia has been demonstrated in many different model systems, and research programs have been designed to tackle this resistance. The importance of hypoxia has been demonstrated clinically where it predicts for worse outcome in the treatment of cancer of the head and neck, uterine cervix, 


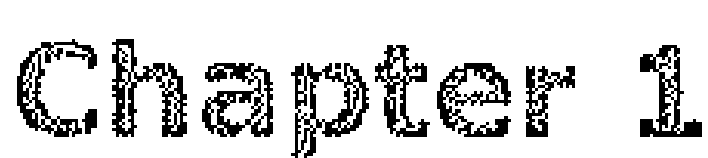

and soft tissue sarcomas $s^{8-10}$. Clinical efforts towards overcoming hypoxia have focused on either eliminating it, for example by breathing high oxygen gas mixtures, or by replacing oxygen with drugs that can sensitize cells to radiation in a similar way to oxygen. These clinical studies have only modestly improved outcome and have been recently reviewed ${ }^{11,12}$.

\subsection{Hypoxia, a molecular resistance to therapy}

As mentioned, several clinical studies have demonstrated an association between the degree of hypoxia within a tumor and the overall patient prognosis. A series of measurements of $\mathrm{pO}_{2}$ values in primary and metastatic tumors of the cervix, breast, head-and-neck and rectum ${ }^{8,13,14}$ have shown that patients with poorer tumor oxygenation exhibit a significantly lower probability of 5-year survival than patients with betteroxygenated tumors. Remarkably, the poorer prognosis for the more hypoxic tumors is valid irrespective of the modality of treatment (radiotherapy or radical surgery). These findings are supported by more recent studies of head-and-neck tumors and soft tissue sarcomas, and strongly suggest that hypoxia fundamentally alters tumor physiology to a more aggressive phenotype ${ }^{15}$.

This appears at first glance to be paradoxical, since hypoxia is a condition unfavorable to cell growth and in many situations can stimulate cell death. The resolution of this paradox lies in our understanding of the biological consequences of hypoxia for the tumor as a whole. In the past decade we have become aware of many important biological changes that occur when cells lack oxygen and the potential contribution of these pathways to the malignant phenotype of tumors. As we will see, the biological response to hypoxia is capable of promoting metastasis, angiogenesis, and selection of cells with diminished apoptotic potential. Consequently, the heterogeneity in oxygenation within a tumor can provide an overall positive advantage to tumor growth. This may explain the persistence of hypoxia, expected to arise very early in tumor 
development, in most patients who present themselves in the clinic despite the fact that these tumors may be highly angiogenic. Of course, in order for hypoxia to produce relevant biological consequences, tumor cells must first be able to adapt and survive extended periods of time without oxygen.

We have hypothesized that the genetic and metabolic changes in cancer cells which drive the development of this "hypoxia tolerance" may be important contributors to the carcinogenic process itself $^{11,}{ }^{16}$. The degree of hypoxia tolerance in a particular tumor may thus be an important determinant of the level of hypoxia which can be sustained within that tumor. We can thus view hypoxia tolerance as a property which provides a 'molecular resistance' towards effective therapy. This realization, along with our understanding of the key cellular pathways activated by hypoxia, leads to the possibility of targeting treatment against this molecular resistance. Several new studies have recently reported on this exciting possibility.

\section{Mechanisms of adaptation to hypoxia}

How does a tumor cell adapt to and survive in a nutrient-deficient and low-oxygen environment? Three key distinct oxygen sensing molecular pathways have emerged over the past several years which contribute to hypoxia tolerance in cancer (figure 1 ). The first is the inhibition or loss of pathways which stimulate cell death in response to hypoxia. This is best exemplified by the 053 pathway, which when functional can result in the rapid death of hypoxic, oncogenically transformed cells. Second is the activation of a conserved transcriptional program mediated in large part by the hypoxia-inducible-factors, HIFs (HIF1, HIF2, HIF3). This is a family of oxygen-sensitive transcription factors that activate more than 60 putative genes (for a review see ${ }^{17}$ ). The HIFs influence tumor response by stimulating angiogenesis, glycolysis, invasion and metastasis. Third is an adaptive response mechanism that involves the regulation of mRNA 


\section{Chapter 1}

translation. How these translational control pathways function and what their consequences are in terms of gene expression and hypoxia tolerance and is subject of this thesis.
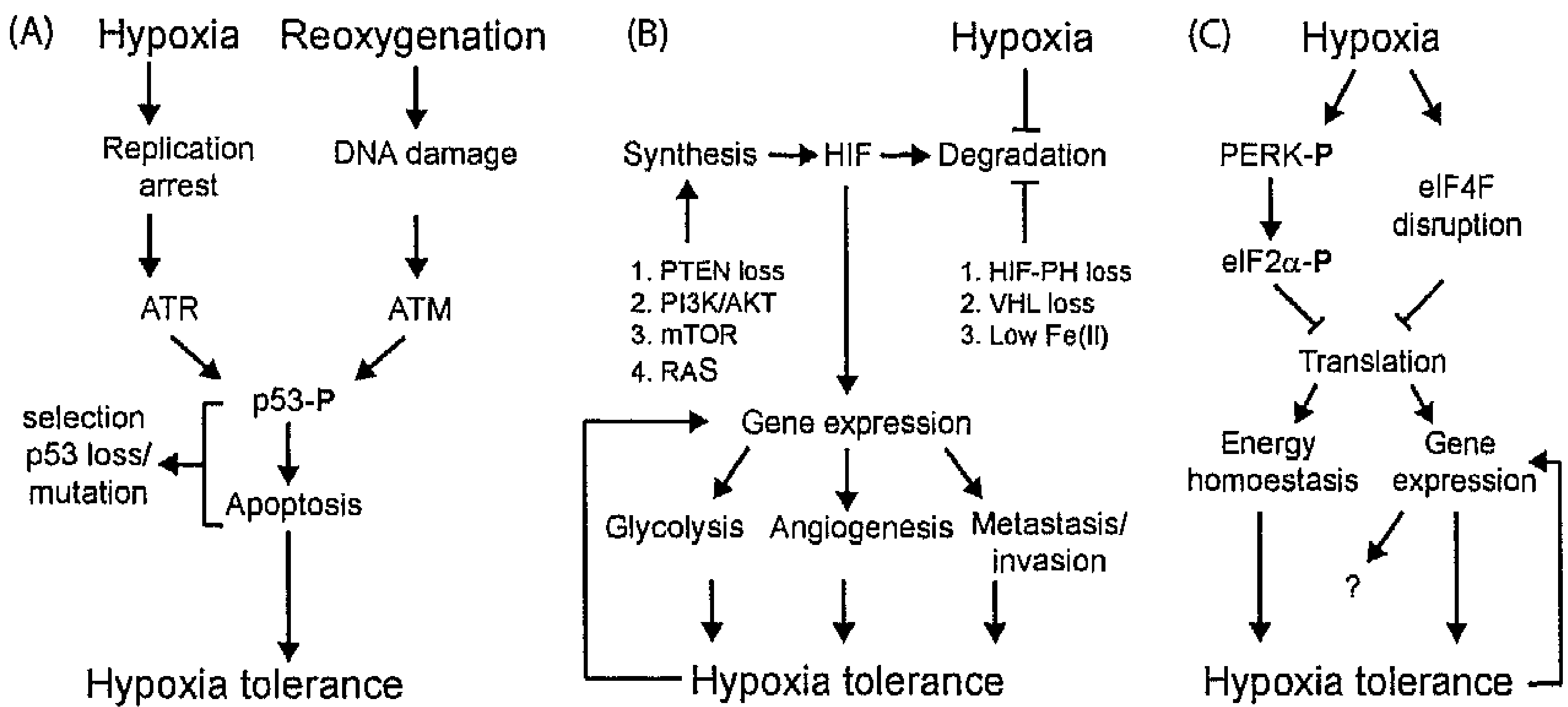

Figure 1. Pathways to hypoxia tolerance. (A) Hypoxia causes the phosphorylation of p53 on Ser15 through ATR, resulting in apoptosis. During reoxygenation p53 phosphorylation is mediated by ATM, leading to apoptosis. This mechanism can provide the basis for selecting cells that have lost their apoptotic sensitivity. (B) Hypoxia and the activation of growth signaling pathways stabilize HIF-1 $\alpha$, thereby activating the transcription of several HRE-target genes leading to increased glycolysis, angiogenesis and metastasis. (C) Hypoxia inhibits transiation, thereby promoting energy homeostasis and increased cell survival during hypoxia.

\subsection{Inhibition of MRNA translation}

Exposure to hypoxia results in rapid and sustained inhibition of mRNA translation. The rate of protein synthesis is reduced by $60-70 \%$ within 1 hour of hypoxic exposure, and rapidly returns to state levels following reintroduction of oxygen ${ }^{18}$. Quantification of mRNA translation through analysis of polysomal RNA indicates that maximal inhibition occurs approximately $2 \mathrm{hr}$ after extreme hypoxia followed by a small recovery by $4 \mathrm{hr}$. However, mRNA translation remains significantly repressed for up to 24 hrs of hypoxia. This phenomenon has been observed in a large panel of cell lines (unpublished data) and appears to be a general response to hypoxia. The reduction in protein synthesis occurs through a HIFindependent, oxygen-sensing pathway which results in direct inhibition of 


\section{General introduction}

the initiation step of mRNA translation ${ }^{16}$. The involvement of a unique oxygen sensor in this pathway provides an explanation for HIFindependent hypoxia-induced biological changes.

mRNA translation is a highly regulated process that is sensitive to a variety of cellular stresses. The inhibition of translation is envisaged to affect tumor cells in two important ways. First, since protein synthesis is extremely energy costly, the inhibition of mRNA translation may act as a cellular survival mechanism by conserving energy demand during this period in which energy production is inefficient. This is exacerbated by the fact that deregulation of MRNA translation appears to be common in tumor cells. It has been known for many years that hypoxia causes a pronounced depression in the rate of oxygen consumption and energy turnover ${ }^{2}, 19$. Studies performed in cell monolayers and in spheroid models of hypoxia have shown that the main ATP-demand pathways in hypoxic or anoxic cells are the $\mathrm{Na}^{+} / \mathrm{K}^{+}$ATPase pump, protein synthesis and degradation, and gluconeogenesis, 19-21. Hochachka et al have calculated that under severe hypoxia, the ATP demand for protein synthesis drops to about $7 \%$ of that of normoxic cells ${ }^{20}$. This drop correlates with a substantial and rapid drop in the rate of protein synthesis, which occurs initially at the levels of translation and later extends at the level of transcription as well. Secondly, the control of mRNA translation is increasingly being viewed as a means of controlling individual protein expression. Mechanisms which regulate global mRNA translation affect individual mRNAs to widely varying extents. The control of translation thus can be viewed as a means of rapidly changing the cellular proteome. The inhibition of overall mRNA translation paradoxically induces the expression of certain proteins that are essential for the adaptation to these conditions. Consequently, hypoxic control of mRNA translation undoubtedly influences individual gene expression and contribute to the hypoxic cell phenotype. 


\section{Regulation of MRNA translation}

In recent years the importance of regulating mRNA translation for gene expression and cell phenotype has been convincingly demonstrated. This process is highly regulated and exquisitely sensitive to various forms of cellular stress as well as to growth factors and other cell signaling processes. It was known 25 years ago that cell proliferation and growth were proportional to the rate of protein synthesis 23. Control of translation has been proven critical in regulating numerous other physiological processes in the cell including: growth stimulation ${ }^{24}$, cell cycle progression $^{25}$, differentiation ${ }^{2 f_{2}}$, ER stress ${ }^{27}$, and oncogenic signaling in cancer $^{28}$.

The process of mRNA translation can be divided into initiation, elongation and termination phases with the initiation phase being the most complex part, underlined by the requirement of over 25 essential factors $^{29,30}$ (see figure 2). The initiation step in which ribosomal subunits are loaded onto the MRNA is also the primary control point for regulating mRNA translation efficiency. In eukaryotes, most translation is 'capdependent' requiring the assembly of an active eukaryotic initiation factor $4 F$ (eIF4F) at the $m^{7} G p p p N$ cap structure present at the $5^{\prime}$ terminus of the mRNA (reviewed in ${ }^{31}$. eIF4F consists Initiation of translation begins following of the $\mathrm{m}^{7} \mathrm{GpppN}$ cap binding protein, eIF4E, a scaffolding protein eIF4G and an ATP-dependent helicase eIF4A. Formation of eIF4F serves as a major control point for regulating the efficiency of mRNA translation by the cell. Assembly of eIF4F at the cap facilitates recruitment of the 435 pre-initiation complex. This pre-initiation complex is composed of the small 40 S ribosomal subunit, the initiation factor elF3, and the ternary complex which consists of eIF2/GTP and the methionine-loaded initiator tRNA, Met-tRNA. Formation of the ternary complex constitutes the second major control point for the cell in regulating the initiation step of translation. A scan through the $5^{\prime}$ UTR of the MRNA by this complex places it at the AUG initiation codon. 
Here, the eIF5 protein stimulates hydrolysis of the eIF2-bound GTP into GDP, which promotes the release of the initiation factors and recruitment of the large 605 ribosomal subunit. Formation of this complete 805 ribosome represents the beginning of the elongation phase of translation ${ }^{32}$.

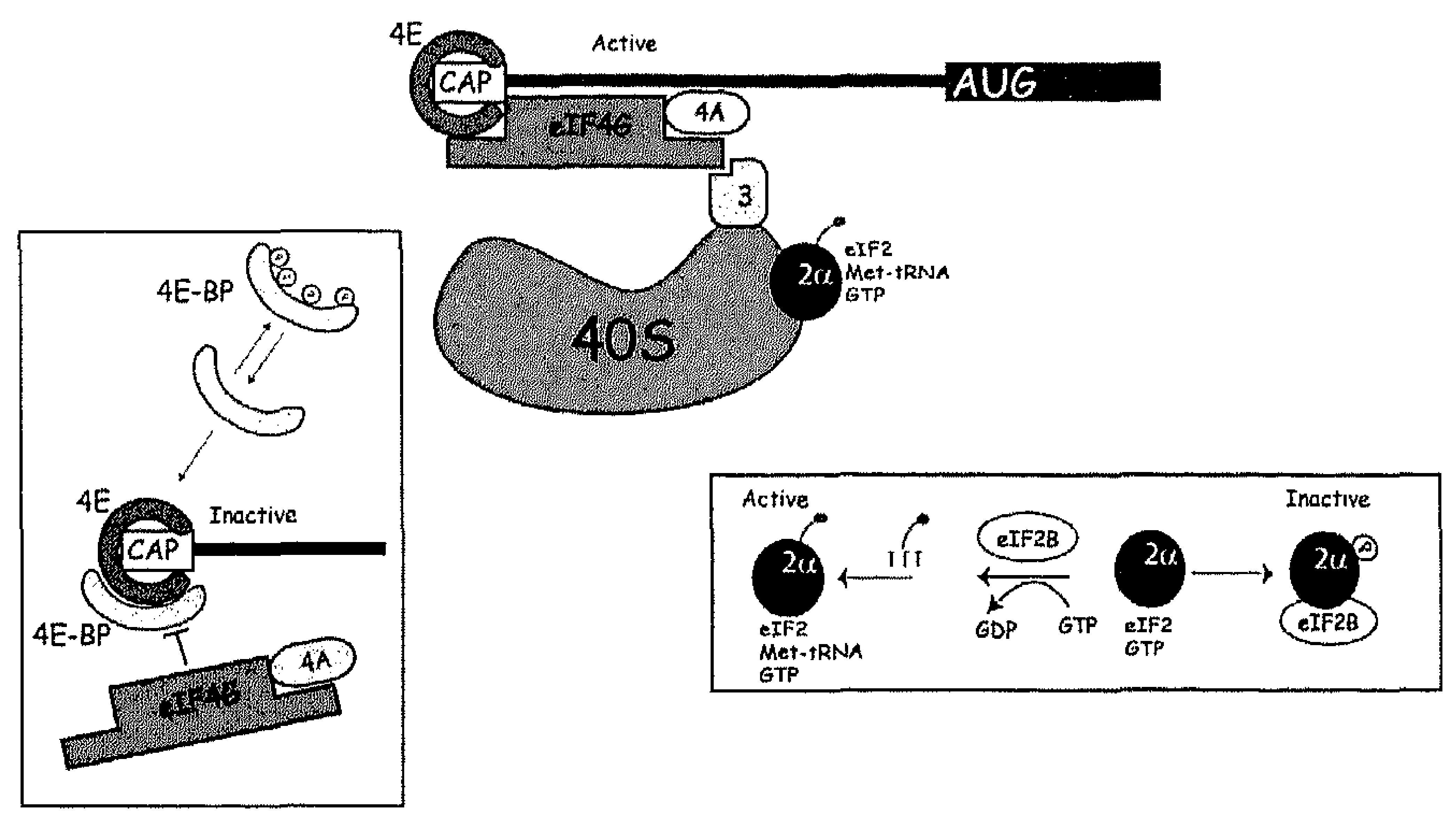

Figure 2. Control of the initiation step of mRNA translation. Initiation of translation begins following assembly of eIF4F (containing the cap-binding protein eIF4E, the scaffold protein eIF4G, and the RNA helicase eIF4A) at the m7GpppN 'cap' structure of the mRNA. Interaction with eIF3 facilitates recruitment of the small subunit of the ribosome together with the ternary complex containing eIF2/Met-tRNA/GTP. This complex then scans through the 5'UTR until it reaches the AUG start site. Here GTP is hydrolyzed releasing eIF2 and allowing recruitment of the large subunit of the ribosome. Control of initiation occurs principally at two steps. The first is through inhibition of eIF4F as a result of competitive binding of the 4E-BPs to eIF4E in their underphosphorylated form. The second is through phosphorylation of eIF $2 \alpha$. Phosphorylation results in binding and inactivation of the GDP/GTP exchange factor eIF2B. 


\subsection{Control of translation through eIF4F}

As mentioned above, the two major control points for stimulating or inhibiting translation are formation of the eIF4F and ternary complexes. Under normal circumstances protein levels of eIF4E are considered to be rate-limiting for assembly of eIF4F and translation initiation. Consequently, regulation of eIF4E availability is arguably the most common mechanism for influencing eIF4F formation. eIF4F assembly is repressed by a family of eIF4E binding proteins (4E-BP1, 4E-BP2 and 4E$B P 3)^{33}, 34$. These small proteins compete with eIF4G for an overlapping binding site within eIF4E. The 4E-BPs are themselves regulated by multiple sequential phosphorylation events on at least 5 known sites. Phosphorylation is controlled in large part through the mammalian target of rapamycin (mTOR) which integrates signals from various pathways (discussed below). Upon hyperphosphorylation, the 4E-BPs lose their affinity for eIF4E, resulting in increased eIF4E:eIF4G binding and stimulation of translation. eIF4G is also subject to direct regulation. During heat-shock, the HSP27 protein binds and sequesters eIF4G in granules to prevent its participation in translation ${ }^{35}$. Furthermore, proteolytic cleavage of eIF4G is reported during apoptosis ${ }^{36}$ and virus infection ${ }^{37}$. The cleavage of eIF4G creates a fragment which lacks the eIF4E-binding motif and is therefore no longer compatible with capdependent translation.

\section{2 mTOR}

mTOR has emerged as an important central regulator of mRNA translation ${ }^{38}$. mTOR is a large protein kinase containing sequence similarity to the catalytic domain of the lipid phosphatidylinositol 3-kinase (PI3K). mTOR stimulates mRNA translation by phosphorylation and inactivation of the eIF4E repressor 4E-BP1, and through phosphorylation and activation of $S 6$ kinase (S6K1). mTOR directly phosphorylates at least two sites in 4E-BP1, Thr37 and Thr46, and may be involved in the phosphorylation of several others. S6K1 and its homolog S6K2 are also 
both direct targets of MTOR. S6K promotes the translation of 5'TOP (terminal oliogpyrimidine tract) mRNA's which contain a polypyrimidine stretch of nucleotides adjacent to the $5^{\prime} \operatorname{cap}^{39-41}$. These mRNAs encode exclusively for components of the translational machinery, including all ribosomal proteins. The stimulation of translation via S6K is indirect and is mediated by other downstream proteins of $56 \mathrm{~K}$ including the ribosomal protein $\mathrm{S} 6$ and the ribosomal recruitment protein eIF $4 \mathrm{~B}^{42}$.

\subsection{Raptor/MLST8}

The upstream regulators of mTOR include important proteins that are sensors of nutrients, energy status, and growth factor signaling. Nutrient sensing by mTOR remains poorly understood, but appears to be mediated by two mTOR binding proteins, Raptor and $\mathrm{MLST}^{43}$. In the absence of nutrients (amino acids) a tight interaction between these 3 proteins is thought to prevent interaction of mTOR with its substrates. In the presence of nutrients a conformational change may partially disrupt this interaction and promote substrate and phosphorylation by $\mathrm{mTOR}^{43,44}$.

\subsection{PI3K/AKT signaling}

Regulation of mTOR and consequently mRNA translation through growth factor signaling pathways is better understood. Insulin and other growth factors lead to phosphorylation of 4E-BP1 and S6K in an mTOR dependent manner. These growth factors signal through the PI3K pathway as evidenced by the fact that inhibition of PI3K blocks 4E-BP1 and 56K phosphorylation in a mTOR sensitive manner ${ }^{45-48}$. Signaling from PI3K to mTOR also involves activation of the AKT kinase via the phosphatidylinositol-dependent kinases PDK1 and PDK2 (see figure 3). AKT activation is negatively regulated by the lipid phosphatase PTEN. AKT appears to be an important mediator of MTOR activity since its activation has results in increased phosphorylation of $4 \mathrm{E}-\mathrm{BP} 1$ and S6K. Furthermore, a dominant negative mutant of AKT blocks insulin mediated phosphorylation of $4 \mathrm{E}-\mathrm{BP} 1^{49}$. 


\subsection{TSC1/2}

Recent work has also shed light on the direct mechanism whereby growth factor signaling through the PI3K/AKT pathway activates mTOR. This mechanism is mediated by the tuberous sclerosis complex (TSC1/TSC2) which is a critical negative regulator of MTOR. Phosphorylation and activation of AKT leads to the inactivation of TSC2, and thus activation of mTOR. It was originally proposed that AKT directly phosphorylates TSC2, however the precise mechanism may be more complicated ${ }^{50}$. Mutations in TSC1 or TSC2 cause deregulation of MTOR activity and an increase in cell and organ size in Drosophila in a manner consistent with mutations in PTEN $^{51-53}$. Mammalian cells deficient for TSC1 or TSC2 fail to downregulate $\mathrm{mTOR}$ function in response to growth factor deprivation and demonstrate constitutively high levels of phosphorylated S6K1 $1^{54-58}$.

As mentioned earlier, the process of mRNA translation is extremely energy costly and it is thus reasonable to assume that mTOR activity may also be sensitive to low cellular energy status. Indeed, activation of mTOR by insulin is inhibited when ATP is reduced by incubation in low glucose media ${ }^{59}$. Recent studies have shown that TSC1/TSC2 also regulates mTOR function in response to changes in energy. AMP-activated protein kinase (AMPK) is a critical mediator of energy metabolism that becomes active when AMP levels are high ${ }^{60}$. AMPK directly phosphorylates and activates $T S C 2^{61}$ leading to $\mathrm{mTOR}$ inhibition and a reduction in $\mathrm{MRNA}$ translation. Correspondingly, cells deficient for TSC1/TSC2 are unable to downregulate mTOR during ATP deprivation ${ }^{61}$. Recent work has shown that activation of AMPK and phosphorylation of TSC2 also requires the LKB1 tumor suppressor protein ${ }^{62,63}$. Under low energy conditions LKB1 phosphorylates and activates AMPK leading to mTOR inhibition. LKB1 and AMPK thus promote maintenance of cellular energy homeostasis through mTORdependent inhibition of mRNA translation. 


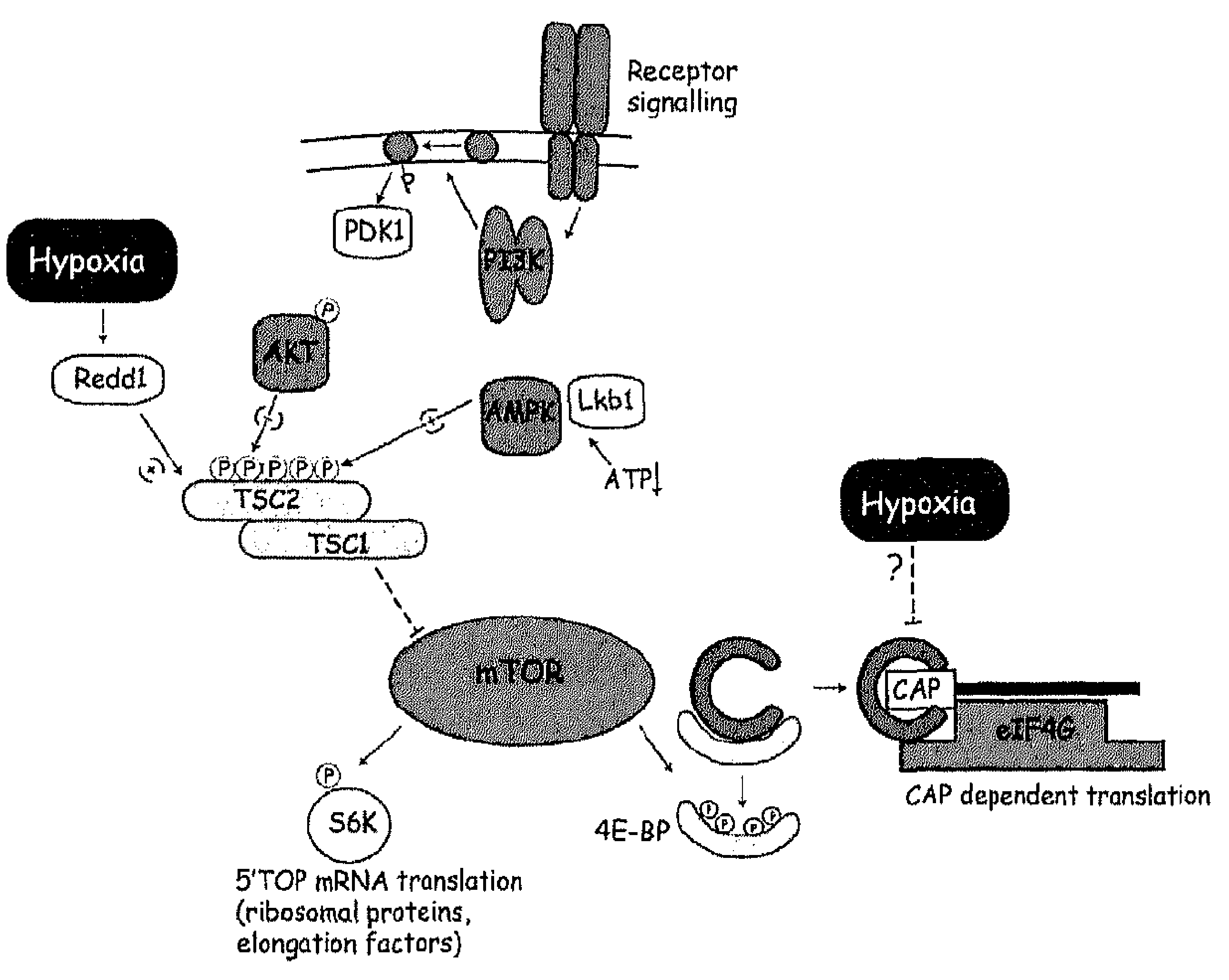

Figure 3. Control of mRNA translation through mTOR. Activation of PI3K upon growth factor receptor signaling leads to an AKT-dependent inhibitory phosphorylation of the TSC2 protein. The TSC1/TSC2 complex is a negative regulator of $\mathrm{MTOR}$, and thus inhibition of TSC2 activates mTOR. Low energy levels inhibit MTOR signaling following an activating phosphorylation of TSC2 by AMPK. Phosphorylation of S6K and 4E-BP1 by mTOR promotes synthesis of the translational machinery and stimulates cap-dependent translation respectively. Hypoxia inhibits mTOR and cap-dependent translation by induction of REDD1 in a TSC2-dependent manner. Hypoxia may additionally inhibit cap-dependent translation through alternative mechanisms.

\subsection{Deregulated eIF4F in cancer}

Considerable evidence supports the concept that changes in mRNA translation contribute to the development of cancer ${ }^{28,64,65}$. For example, several genes that participate in signaling pathways upstream of mTOR are frequently altered in cancer. This includes the commonly disrupted PI3K/AKT pathway. Mutations in the tumor suppressor gene PTEN ${ }^{66,} 67$ result in deregulation of AKT and subsequent activation of mTOR and 


\section{Chapter 1}

mRNA translation. Similarly, receptors such as HER2/Neu which signal to this pathway are also frequent targets of amplification or mutation in cancer ${ }^{68}$. Mutations in either TSC1 or TSC2 results in formation of benign tumors called hamartomas which consist of multiple cell types ${ }^{69}$. Mutations in the LBK1 tumor suppressor gene, which regulates TSC1/TSC2 in response to reductions in energy levels, causes Peutz-Jeghers syndrome $(\mathrm{PJS})^{70}$. This disease is characterized by the development of hamartomas and various neoplasms, which results as a direct consequence of mTOR deregulation ${ }^{71}$.

Downstream of mTOR several studies linking eIF4E to cancer provide more direct evidence for the role of mRNA translation in transformation and tumor progression. Activation of mTOR stimulates translation by increasing eIF4E availability, eIF4E is frequently over-expressed in human cancer and is can directly transform immortalized $3 T 3$ fibroblasts ${ }^{65}, 72$. Recently, two genetic studies in mice have demonstrated a clear role for eIF4E in tumorigenesis ${ }^{73},{ }^{74}$. Transgenic mice overexpressing eIF4E showed significantly increased tumorigenesis ${ }^{73}$. Modest overexpression of eIF4E (2.5-fold) also considerably accelerated lymphomagenesis in the EH-Myc transgenic B-cell lymphoma model ${ }^{73}$, 74 . In this system eIF4E accelerated tumor development through the inhibition of apoptosis ${ }^{74}$.

\subsection{Control of translation through eIF 2}

The second major control point for regulation of translation involves formation of the eIF2/GTP/Met-tRNA ternary complex. Upon the start of protein synthesis, the eIF5 protein stimulates hydrolysis of the eIF2-bound GTP into GDP, which results in the release of the initiation factors from the ribosome. Thus, in order to load another ribosome onto the RNA and initiate an additional round of protein synthesis, the exchange of eIF2/GDP for GTP is required. This reaction is catalyzed by the initiation factor eIF2B. However, this exchange reaction is inhibited when the eIF2a subunit becomes phosphorylated at ser51. Phosphorylation of eIF $2 \alpha$ does not prevent the binding of eIF2B, but it does prevent the exchange of GDP 
for GTP. Consequently, eIF2B remains bound to the phosphorylated and inactive eIF2 complex. Because the eIF2B subunit exists in significantly lower amounts than eIF $2 \alpha$ (about $20-30 \%$ of eIF $2 \alpha$ a less than complete phosphorylation of eIF $2 \alpha$ molecules is sufficient to inhibit the exchange activity of eIF2B. Although phosphorylation of eIF2 $\alpha$ results in a general inhibition of mRNA translation, it also leads to increased translation of specific mRNAs as will be discussed later.

At least 4 distinct kinases have been shown to phosphorylate eIF $2 \alpha$ at ser51 and to repress translation in response to stress (reviewed $\mathrm{in}^{75,76}$ ). These are: (i) PKR, an interferon inducible, double-stranded RNA-activated protein kinase important during virus infection ${ }^{77}$; (ii) HRI, a heme regulated inhibitor of translation found predominantly in erythroid cells ${ }^{78}$; (iii) GCN2, a kinase originally identified in yeast which is activated upon nutrient (amino acid) deprivation ${ }^{79}$; and (iv) PERK, an endoplasmic reticulum resident kinase which participates in a conserved pathway known as the unfolded protein response (UPR) ${ }^{80}$. During the UPR, PERK is activated by autophosphorylation resulting in phosphorylation of eIF2 $\alpha$ and inhibition of translation. All of the kinases which phosphorylate eIF $2 \alpha$ are considered to be "stress-responsive" and are thought to play key roles in the adaptation of cells to adverse environments. PERK resides in the endoplasmic reticulum (ER) membrane with its kinase domain in the cytoplasmic side and is activated by ER stress ${ }^{80}$. PERK knockout MEFs are unable to phosphorylate eIF2 $\alpha$ in response to ER stress ${ }^{81}$ and correspondingly display increased sensitivity to agents that cause ER stress $^{81}$. Bertolotti et al demonstrated that the lumenal domain of PERK is bound to BiP/GRP78 and that perturbation of protein folding leads to a dissociation of these two proteins and activation of PERK ${ }^{82}$. Therefore, a model has emerged in which BiP acts as a repressor of PERK activation under normal conditions. Upon an increase in protein misfolding, relief of this repression leads to PERK activation, eIF2 $\alpha$ phosphorylation and inhibition of translation. 


\section{Mechanistic basis and importance of translational control during hypoxia}

During hypoxia mRNA translation is rapidly inhibited as evidenced by a massive reduction in ribosome associated or 'polysomal' RNA (see figure 4). Why is translational regulation employed under conditions of low oxygen availability? If one views the useful properties of translational control for the cell in general as stated by Mathews et al. ${ }^{83}$ (e.g., directness and rapidity, reversibility, fine control, etc.) it becomes evident that from a teleological perspective that several of these properties would be beneficial under conditions of hypoxia. Transcriptional control is exerted at a much earlier step in the process of gene expression compared to translational control, and thus a change in protein levels requires much longer if regulated at the level of transcription, especially if the mRNA of the particular protein is abundant and/ or has a long half-life. Conversely, if the cell becomes re-oxygenated, translational control is a very efficient and rapid mechanism for quickly elevating protein synthesis levels to meet cellular demands. In tumors, fluctuations in oxygenation from near normal to near anoxia can occur with a periodicity of 15 to 20 minutes, clearly too short a time for transcriptional adaptation ${ }^{84}$. The mechanisms which inhibit translation during hypoxia are multi-factorial and involve both of the primary control points used to regulate the initiation step of this process. Clues as to the mechanistic basis and importance of this inhibition have also recently become available.

\subsection{Control of translation during hypoxia through eIf $2 \alpha$}

We previously reported that exposure of human diploid fibroblasts, minimally transformed cells, and various tumor cell lines to hypoxia leads to phosphorylation of eIF $2 \alpha^{16,85}$ and inhibition of protein synthesis. Importantly, this response is independent of HIF-1 $\alpha$ accumulation as both HIF- $1 \alpha^{+/+}$and HIF-1 $\alpha^{-/}$MEFs similarly phosphorylate eIF2 $\alpha$ in response to hypoxia ${ }^{16}$. Phosphorylation of eIF2 $\alpha$ occurs within 1 hour of hypoxic 


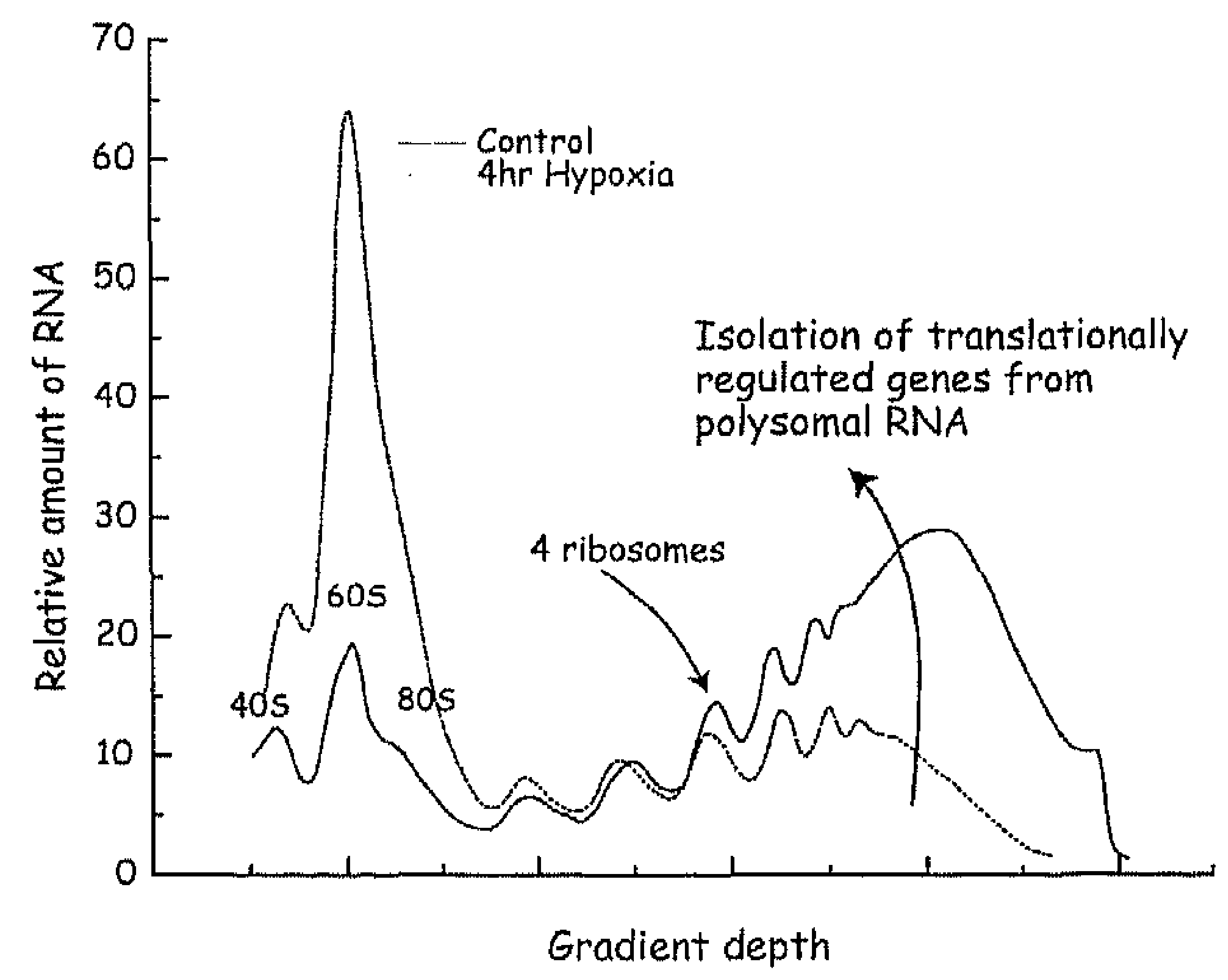

Figure 4. Reduction in polysomal RNA during hypoxic exposure. MRNA translation can be assessed by examining the association of ribosomes with mRNA. DU145 human prostate cancer cells were exposed to aerobic conditions or 4 hrs of hypoxia. RNA was then separated on the basis of size through a sucrose gradient. The first peak represents free 405 ribosomal subunits, the second free 605 subunits, and the third complete $80 \mathrm{~S}$ ribosomes. Each subsequent peak represents 'polysomal' mRNA with 2, 3, 4, 5, etc ribosomes attached to the mRNA. Hypoxia results in an inhibition of translation as evidenced by a dramatic reduction in polysomal RNA and a shift to mRNAs that are less efficiently translated (fewer ribosomes). Transcripts that remain actively translated during hypoxia can be identified by microarray analysis of the remaining polysomal RNA.

exposure and before any significant changes in transcription or cellular energy status. Furthermore, the phosphorylation which occurs during hypoxia is completely reversible upon reoxygenation. As outlined above, the phosphorylation of eIF $2 \alpha$ prevents the exchange of GDP for GTP by eIF2B and thus prevents formation of an active ternary complex which can participate in translation initiation. Expression of a transdominant, nonphosphorylatable mutant allele of eIF $2 \alpha$ attenuates the repression of protein synthesis under hypoxia. We also demonstrated that the endoplasmic reticulum resident eIF $2 \alpha$ kinase, PERK, becomes hyperphosphorylated upon hypoxic stress, and overexpression of wt-PERK increases the levels of hypoxia-induced phosphorylation of eIF $2 \alpha$. Cells 


\section{Chapter 1}

stably expressing a dominant-negative PERK allele and mouse embryonic fibroblasts (MEFs) with a homozygous deletion of PERK exhibit attenuated phosphorylation of eIF $2 \alpha$ and reduced inhibition of protein synthesis in response to hypoxia. MEFs lacking PERK expression show not only attenuated inhibition of translation but also reduced survival after prolonged exposure to hypoxia ${ }^{16}$. Similar results were obtained using human tumor cells expressing a non-phosphorylatable eIF2 $\alpha$ construct. These results indicate that adaptation of cells to hypoxic stress requires activation of PERK and phosphorylation of eIF2 $\alpha$ and suggest that the mechanism of hypoxia-induced translational attenuation may be linked to ER stress ${ }^{16}$.

Phosphorylation of eIF $2 \alpha$ and inhibition of protein synthesis has also been demonstrated to occur in response to ischemia-reperfusion injury in neuronal tissue ${ }^{86-89}$. However, in these cases eIF $2 \alpha$ phosphorylation is observed only upon reoxygenation. While apparently similar to hypoxia, ischemia has distinct properties because of the more acute nature of the stress, the vulnerability of the neurons to the reperfusion and the fact that ischemia-reperfusion has a strong component of free-radical damage, which is not present in all modes of chronic hypoxia in tumor cells. Indeed, in our preliminary studies, not only did we observe eIF2 $\alpha$ phosphorylation in the absence of any reoxygenation, but also reoxygenation induced a rapid dephosphorylation of eIF $2 \alpha$. Therefore, the regulation of eIF $2 \alpha$ phosphorylation by hypoxia in tumor cells appears to be governed by distinct pathways not present or utilized in ischemiareperfusion. To our knowledge, this is the first demonstration of eIF $2 \alpha$ phosphorylation in response to hypoxia in non-neuronal cells.

\subsection{Hypoxia and the UPR}

The global decrease in MRNA translation is reminiscent of the heat-shock response when cells are exposed to temperatures of over $43^{\circ} \mathrm{C}^{90-93}$. Another striking similarity between the two responses is that they are paradoxically characterized by a robust upregulation of a few proteins 
whose main function is to protect the cell. In fact, some of the stressresponse proteins that are upregulated in the heat shock response, like heat shock protein 70 (HSP70) and glucose-regulated protein/binding protein (GRP78/BiP) are also upregulated under hypoxia ${ }^{94-99}$ Importantly, inhibition of BiP expression results in increased cell death in cells exposed to hypoxic stress ${ }^{100}$. A likely explanation for the similarity between hypoxia and this heat-shock response is the induction of an evolutionarily conserved pathway known as the unfolded protein response (UPR).

Inhibition of mRNA translation through the phosphorylation of eIF2 $\alpha$ at Ser51 is an evolutionarily conserved response to a multitude of cellular stresses and has been termed the Integrated Stress Response (ISR) ${ }^{101}$. Other stresses which can activate the ISR include amino acid withdrawal and a variety of agents that activate endoplasmic reticulum (ER) stress. The lumen of the ER is an active site of protein folding in which all secretory and membrane-bound proteins are post- or co-translationally translocated into. The ER maintains a unique oxidizing environment necessary for the proper folding of these proteins, and is also important for posttranslational modifications such as glycosylation ${ }^{102}$. ER stresses, such as perturbations in calcium storage, redox status and inhibition of protein glycosylation, compromise the ER's capacity to correctly fold proteins, thereby leading to an accumulation of unfolded proteins within the $E R^{103}$. This imbalance between the ER's folding capacity and its protein load induces the UPR ${ }^{104}$. The UPR constitutes three unique signaling pathways, one of which is characterized by activation of the ISR through PERK and subsequent phosphorylation of eIF $2 \alpha^{104}$ (see figure 3 ). The activation of the UPR not only reduces ER protein load by inhibiting the translation of new proteins through the ISR, but also induces the expression of UPR target genes responsible for restoring ER homeostasis.

In mammalian cells, the UPR is mediated by three ER transmembrane proteins: PERK, IRE1 and ATF6. Each of these proteins has a lumenal domain that is negatively regulated by the ER chaperone BiP/GRP78 ${ }^{82}$. In unstressed cells, BiP binds the lumenal domains of all three proteins, 
Chapter 1

maintaining them in their inactive states. Upon accumulation of unfolded proteins, BiP dissociates from PERK, IRE1 and ATF6 to promote protein folding. Consequently, each of these three ER proteins activates a distinct pathway of the UPR, inducing a global translational attenuation as well as the transcriptional activation of UPR target genes. Such genes encode proteins involved in protein folding, glycosylation, calcium homeostasis and protein degradation ${ }^{102}$.

Several lines of evidence demonstrate that hypoxia is also able to activate the UPR and that cancer cells utilize the UPR as a survival mechanism under conditions of low oxygen. First of all, as described above, hypoxia activates the ISR through activation of PERK and phosphorylation of eIF2 $\alpha$. This leads to attenuation of mRNA translation and a reduction in the protein content of the ER. This inhibition also paradoxically leads to increased expression of several proteins including the transcription factor ATF4. ATF4 plays an important role by transcriptionally regulating several other genes including $\mathrm{CHOP}$ (GADD153) and GADD34, and may be particularly important in preventing oxidative stress ${ }^{101}$. CHOP is a transcription factor implicated in activating apoptosis, whereas GADD34 is part of a negative feedback loop that promotes the dephosphorylation of eIF2 $\alpha$ and recovery from the ER stress response ${ }^{105}$. Blais et.al. showed that ATF4 is similarly induced during hypoxia in a PERK and mRNA translation dependent manner ${ }^{85}$. Both of the ATF4 target genes GADD34 and CHOP are also induced by hypoxia. Interestingly, primary human breast tumors also demonstrate higher ATF4 and $\mathrm{CHOP}$ expression as compared to normal tissues. The expression of ATF4 and CHOP has also been reported to occur in tumors in areas adjacent to necrosis and which are thought to be hypoxic ${ }^{106}$. New data also demonstrates that activation of the PERK-eIF2 $\alpha$ pathway during hypoxia is necessary for efficient tumor growth in vivo. Transformed MEFs lacking PERK are unable to activate this pathway and produce much smaller tumors than their wild-type counterparts. The tumors grow slowly because the cells are unable to survive hypoxia within the tumor 
microenvironment ( $\mathrm{Bi}, \mathrm{M}$. et al. submitted). More importantly, human tumor cells expressing a dominant-negative PERK allele also show inhibited tumor growth in vivo, with hypoxic areas of the tumor colocalizing with areas of apoptosis ( $\mathrm{Bi}, \mathrm{M}$. et al. submitted). Thus the UPR, activated under hypoxic conditions in tumors, may represent an important survival mechanism that contributes to hypoxia tolerance and cancer growth. There is also some evidence that hypoxia activates the other two arms of the UPR pathway mediated by ATF6 and IRE1. Upon activation of the UPR, ATF6 is known to be cleaved into a smaller fragment which is released from the ER. This cleaved form translocates to the nucleus where it functions as an active transcription factor. Hypoxia also induces ATF6 cleavage and nuclear localization in HeLa cells (unpublished data). Also, there is recent evidence that the UPR pathway controlled by IRE1 is activated and important during hypoxia. Activated IRE1 contains a nuclease activity that removes 26 bases from the XBP1 transcript. This frameshift allows the synthesis of a much more potent transcription factor compared with the unspliced form of the protein. XBP1 activates the transcription of several genes involved in the UPR that contain an ER stress response element (ERSRE) within their promoters. This includes genes such as BiP and protein disulfide isomerase (PDI) both of which are also activated by hypoxia. A recent report shows that MEFs derived from XBP1 knockout mice have increased levels of apoptosis and reduced clonogenic survival in response to hypoxia ${ }^{107}$. Loss of XBP1 also inhibited tumor growth due to their reduced ability to survive in a hypoxic microenvironment. These studies thus implicate the IRE1/XBP1 arm of the UPR as an important factor for hypoxic stress and tumor growth. These data suggest that all three arms of the UPR are activated during hypoxia in a time- and oxygen concentration-dependent manner. Disruption of either of the pathways controlled by PERK or IRE1 sensitizes cells to hypoxia and impairs tumor growth. 


\section{Chapter 1}

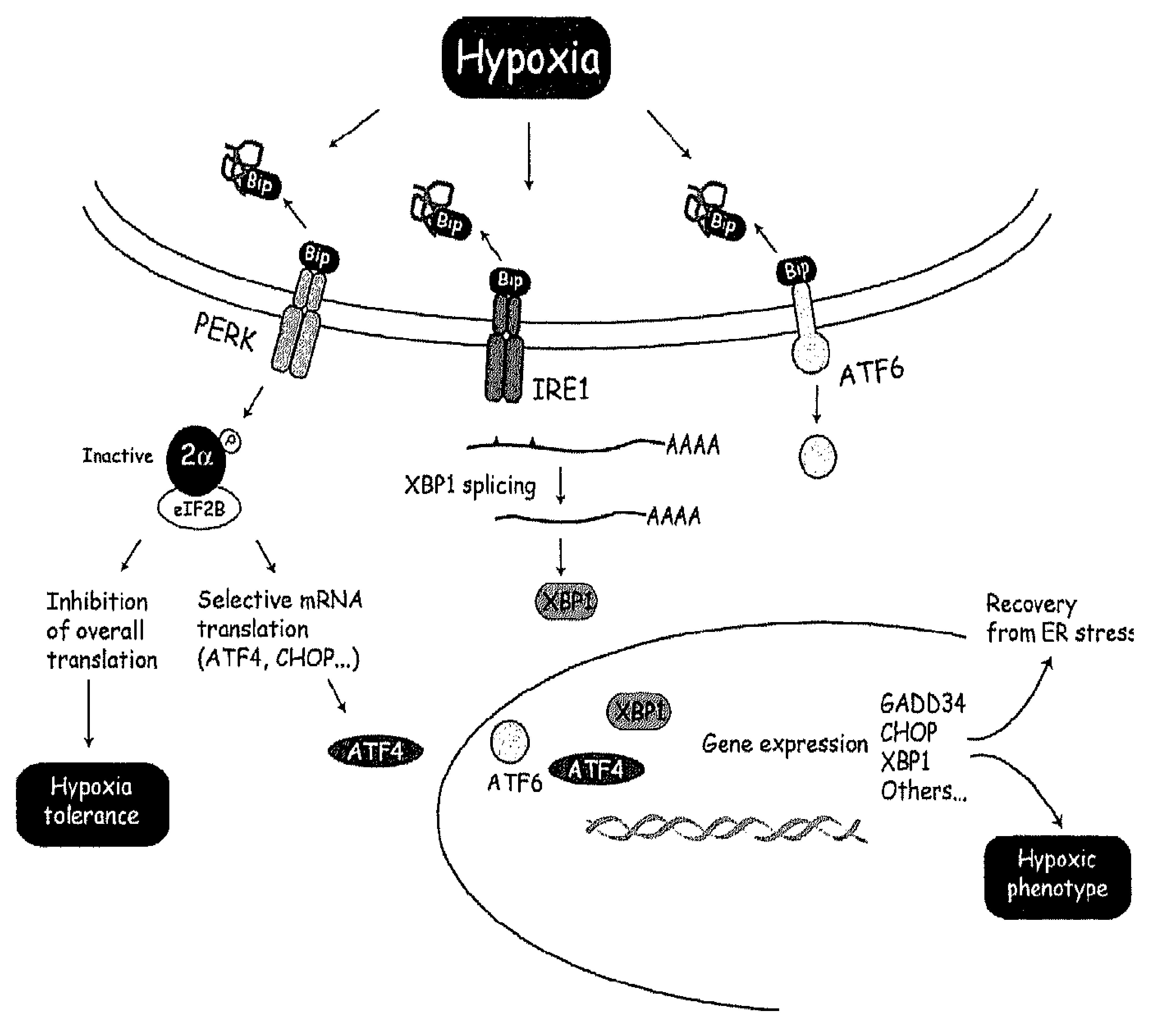

Figure 5. Hypoxia and the UPR. Evidence suggests that hypoxia activates a conserved ER stress response program known as the UPR. This response is mediated by three ER transmembrane proteins PERK, IRE1, and ATF6. The activation of PERK and subsequent phosphorylation of eIF2 $\alpha$ is the best characterized arm of this response during hypoxia. Phosphorylation of eIF2 $\alpha$ leads to a general inhibition of overall mRNA translation, but stimulates the translation of a specific subset of proteins including ATF4. IRE1 activation removes a 26nucleotide region of the XBP1 mRNA resulting in the formation of a potent transcription factor. Finally, ATF6 is cleaved, processed in the golgi, and translocates to the nucleus where it induces gene expression. 
An unresolved issue which will be the focus for future research regards the mechanism by which the UPR is activated during hypoxia. The unique oxidizing environment of the ER provides some clues.

\subsection{Control of translation during hypoxia through eIF4F}

Our group has evidence that the observed inhibition of translation during hypoxia cannot be fully accounted for by the phosphorylation of eIF $2 \alpha$. Phosphorylation of eIF $2 \alpha$ reaches a maximum during hypoxia and then declines even though translation rates as assessed by polysomal mRNA remain low. Furthermore, we have recent evidence that cells derived from a mouse in which a non-phosphorylatable allele of eIF $2 \alpha$ was knocked-in $(\mathrm{S} 51 \mathrm{~A})^{108}$ still display a substantial (albeit reduced) inhibition of translation. Another candidate mechanism that could account for this discrepancy is disruption of the cap-binding protein complex eIF4F. We have preliminary data suggesting that hypoxia also disrupts the eIF4F complex through multiple mechanisms including dephosphorylation of the 4E-BPs (unpublished data). This 'second' pathway of inhibiting translation occurs only after longer periods of exposure to hypoxia (4-16 h) and provides a possible mechanism for the sustained down-regulation of protein synthesis that needs further investigation.

\section{Hypothesis and outline of this thesis}

As outlined in the previous paragraphs, hypoxia has a profound effect on protein synthesis through inhibition of mRNA translation. Our hypothesis was that hypoxia influences translational control through two independent pathways that are each important contributors to gene expression and hypoxic cell phenotype. In chapter $\mathbf{2}$ we investigated the first part of this hypothesis by characterizing the role and requirement of upstream signaling pathways that regulate translation initiation during hypoxia. In this study, we also demonstrated the importance of these regulatory pathways on the activation of several hypoxia regulated genes. 
The second part of our hypothesis relates to the effects of translational control by hypoxia on gene expression. We set out to identify and characterize specific genes that produce mRNAs which have an ability to remain translated under conditions in which overall protein synthesis is repressed. We reviewed the current understanding of the mechanisms leading to selective mRNA translation and gene expression during hypoxia in chapter 3. In chapter 4 we assessed changes in the hypoxic gene expression using a proteomic approach combined with genome-wide analysis of well translated transcripts. The relative contributions of translational control to gene expression were further evaluated in chapter 5. In chapter 6 and chapter 7 we describe the novel regulation of two important hypoxia regulated genes, CITED2 and carbonic anhydrase 9 (CA9) which are controlled through hypoxia dependent changes in mRNA translation.

The last part of our hypothesis relates to the influence of translational control on the hypoxic cell phenotype. We tested this aspect of our hypothesis by initiating studies to determine if the translational control pathways are good candidates for the development of novel hypoxia sensitizing drugs. For this we generated an isogenic cell model in which we can modulate the translational control pathways in an inducible manner. The effect of targeting these pathways on hypoxia tolerance are described in chapter 8. 


\section{References}

1. American Cancer Society: Cancer Facts and Figures 2007, http://www.cancer.org.

2. Koch C]: Oxygen effects in radiobiology, Adv Exp Med Biol 1982, 157:123144

3. Sutherland RM, Eddy HA, Bareham B, Reich K, Vanantwerp D: Resistance to adriamycin in multicellular spheroids, Int J Radiat Oncol Biol Phys 1979, 5:1225-1230

4. Sutherland RM, Ausserer WA, Murphy BJ, Laderoute KR: Tumor Hypoxia and Heterogeneity: Challenges and Opportunities for the Future, Semin Radiat Oncol 1996, 6:59-70

5. Luk CK, Sutherland RM: Influence of growth phase, nutrition and hypoxia on heterogeneity of cellular buoyant densities in in vitro tumor model systems, Int J Cancer 1986, 37:883-890

6. Brown JM, Giaccia AJ: The unique physiology of solid tumors: opportunities (and problems) for cancer therapy, Cancer Res 1998, 58:1408-1416

7. Chaplin DJ, Hill SA: Temporal heterogeneity in microregional erythrocyte flux in experimental solid tumours, $\mathrm{Br}$ J Cancer 1995, 71:1210-1213

8. Hockel $M$, Schlenger $K$, Aral B, Mitze M, Schaffer U, Vaupel P: Association between tumor hypoxia and malignant progression in advanced cancer of the uterine cervix, Cancer Res 1996, 56:4509-4515

9. Brizel DM, Sibley GS, Prosnitz LR, Scher RL, Dewhirst MW: Tumor hypoxia adversely affects the prognosis of carcinoma of the head and neck, Int J Radiat Oncol Biol Phys 1997, 38:285-289

10. Nordsmark M, Alsner J, Keller J, Nielsen OS, Jensen OM, Horsman MR, Overgaard J: Hypoxia in human soft tissue sarcomas: adverse impact on survival and no association with p53 mutations, Br J Cancer 2001, 84:10701075

11. Wouters BG, Koritzinsky M, Chiu RK, Theys J, Buijsen J, Lambin P: Modulation of cell death in the tumor microenvironment, Semin Radiat Oncol 2003, 13:31-41

12. Wouters BG, Weppler SA, Koritzinsky M, Landuyt W, Nuyts S, Theys J, Chiu RK, Lambin P: Hypoxia as a target for combined modality treatments, Eur J Cancer 2002, 38:240-257

13. Hockel M, Knapstein PG, Hohenfellner R, Rosler HP, Kutzner ]: [Combined surgical and radiotherapeutic treatment of pelvic wall recurrences: report of experiences after 3 years], Geburtshilfe Frauenheilkd 1993, 53:169-176

14. Vaupel $P$, Thews $O$, Kelleher DK, Hoeckel M: Oxygenation of human tumors: the Mainz experience, Strahlenther Onkol 1998, 174 Suppl 4:6-12

15. Vaupel $P$, Hoeckel $M$ : Predictive power of the tumor oxygenation status, $A d v$ Exp Med Biol 1999, 471:533-539

16. Koumenis $C$, Naczki $C$, Koritzinsky $M$, Rastani S, Diehl A, Sonenberg $N$, Koromilas A, Wouters BG: Regulation of protein synthesis by hypoxia via activation of the endoplasmic reticulum kinase PERK and phosphorylation of the translation initiation factor elF2alpha, Mol Cell Biol 2002, 22:7405-7416

17. Semenza GL: Targeting HIF-1 for cancer therapy, Nat Rev Cancer 2003, 3:721-732

18. Kraggerud SM, Sandvik JA, Pettersen EO: Regulation of protein synthesis in human cells exposed to extreme hypoxia, Anticancer Res 1995, 15:683-686

19. Sutherland R, Freyer J, Mueller-Klieser W, Wilson R, Heacock C, Sciandra J, Sordat B: Cellular growth and metabolic adaptations to nutrient stress 


\section{Chapter 1}

environments in tumor microregions, Int J Radiat Oncol Biol Phys 1986, 12:611-615

20. Hochachka PW, Buck LT, Doll CJ, Land SC: Unifying theory of hypoxia tolerance: molecular/metabolic defense and rescue mechanisms for surviving oxygen lack, Proc Natl Acad Sci U S A 1996, 93:9493-9498

21. Hochachka PW, Monge C: Evolution of human hypoxia tolerance physiology, Adv Exp Med Biol 2000, 475:25-43

22. Baxter GC, Stanners CP: The effect of protein degradation on cellular growth characteristics, J Cell Physiol 1978, 96:139-145

23. Johnson LF, Williams JG, Abelson HT, Green H, Penman S: Changes in RNA in relation to growth of the fibroblast. III. Posttranscriptional regulation of mRNA formation in resting and growing cells, Cell 1975, 4:69-75

24. Nielsen FC, Ostergaard L, Nielsen J, Christiansen J: Growth-dependent translation of IGF-II mRNA by a rapamycin-sensitive pathway, Nature 1995, 377:358-362

25. Rosenwald IB, Setkov NA, Kazakov VN, Chen JJ, Ryazanov AG, London IM, Epifanova OI: Transient inhibition of protein synthesis induces expression of proto-oncogenes and stimulates resting cells to enter the cell cycle, Cell Prolif 1995, 28:631-644

26. Sheets MD, Fox CA, Hunt $T$, Vande Woude G, Wickens $M$ : The 3'untranslated regions of c-mos and cyclin mRNAs stimulate translation by regulating cytoplasmic polyadenylation, Genes Dev 1994, 8:926-938

27. Novoa I, Zhang $Y$, Zeng $H$, Jungreis $R$, Harding HP, Ron D: Stress-induced gene expression requires programmed recovery from translational repression, Embo J 2003, 22:1180-1187

28. Rajasekhar VK, Viale A, Socci ND, Wiedmann M, Hu X, Holland EC: Oncogenic Ras and Akt signaling contribute to glioblastoma formation by differential recruitment of existing mRNAs to polysomes, Mol Cell 2003, 12:889-901

29. Pestova TV, Kolupaeva VG, Lomakin IB, Pilipenko EV, Shatsky IN, Agol VI, Hellen CU: Molecular mechanisms of translation initiation in eukaryotes, Proc Natl Acad Sci U S A 2001, 98:7029-7036

30. Preiss $T, M W H$ : Starting the protein synthesis machine: eukaryotic translation initiation, Bioessays 2003, 25:1201-1211

31. Gingras $A C$, Raught $B$, Sonenberg N: eIF4 initiation factors: effectors of mRNA recruitment to ribosomes and regulators of translation, Annu Rev Biochem 1999, 68:913-963

32. Kozak M: Initiation of translation in prokaryotes and eukaryotes, Gene 1999, 234:187-208

33. Pause A, Belsham GJ, Gingras AC, Donze O, Lin TA, Lawrence JC, 3r., Sonenberg $\mathrm{N}$ : Insulin-dependent stimulation of protein synthesis by phosphorylation of a regulator of 5'-cap function, Nature 1994, 371:762-767

34. Poulin $F$, Gingras AC, Olsen $H$, Chevalier $S$, Sonenberg N: 4E-BP3, a new member of the eukaryotic initiation factor $4 \mathrm{E}-$ binding protein family, J Biol Chem 1998, 273:14002-14007

35. Cuesta R, Laroia G, Schneider RJ: Chaperone hsp27 inhibits translation during heat shock by binding eIF4G and facilitating dissociation of capinitiation complexes, Genes Dev 2000, 14:1460-1470

36. Marissen WE, Gradi A, Sonenberg N, Lloyd RE: Cleavage of eukaryotic translation initiation factor 4GII correlates with translation inhibition during apoptosis, Cell Death Differ 2000, 7:1234-1243

37. Gradi A, Svitkin YV, Imataka $H$, Sonenberg $N$ : Proteolysis of human eukaryotic translation initiation factor eIF4GII, but not eIF4GI, coincides with 
the shutoff of host protein synthesis after poliovirus infection, Proc Natl Acad Sci U S A 1998, 95:11089-11094

38. Hay N, Sonenberg N: Upstream and downstream of mTOR, Genes Dev. 2004, 18:1926-1945

39. Jefferies HB, Fumagalli S, Dennis PB, Reinhard C, Pearson RB, Thomas G: Rapamycin suppresses 5'TOP mRNA translation through inhibition of p70s6k, Embo J 1997, 16:3693-3704

40. Jefferies HB, Reinhard C, Kozma SC, Thomas G: Rapamycin selectively represses translation of the "polypyrimidine tract" mRNA family, Proc Natl Acad Sci U S A 1994, 91:4441-4445

41. Terada N, Patel HR, Takase K, Kohno K, Nairn AC, Gelfand EW: Rapamycin selectively inhibits translation of mRNAs encoding elongation factors and ribosomal proteins, Proc Natl Acad Sci U S A 1994, 91:11477-11481

42. Manzella JM, Rychlik W, Rhoads RE, Hershey JW, Blackshear PJ: Insulin induction of ornithine decarboxylase. Importance of mRNA secondary structure and phosphorylation of eucaryotic initiation factors eIF-4B and eIF4E, J Biol Chem 1991, 266:2383-2389

43. Kim DH, Sarbassov DD, Ali SM, Latek RR, Guntur KV, Erdjument-Bromage $H_{\text {, }}$ Tempst $P$, Sabatini DM: GbetaL, a positive regulator of the rapamycinsensitive pathway required for the nutrient-sensitive interaction between raptor and MTOR, Mol Cell 2003, 11:895-904

44. Kim DH, Sarbassov DD, Ali SM, King JE, Latek RR, Erdjument-Bromage $H$, Tempst $P$, Sabatini DM: mTOR interacts with raptor to form a nutrientsensitive complex that signals to the cell growth machinery, Cell 2002, 110:163-175

45. Brunn GJ, Williams J, Sabers C, Wiederrecht G, Lawrence JC, Jr., Abraham RT: Direct inhibition of the signaling functions of the mammalian target of rapamycin by the phosphoinositide 3-kinase inhibitors, wortmannin and LY294002, Embo J 1996, 15:5256-5267

46. Cheatham B, Vlahos CJ, Cheatham L, Wang L, Blenis J, Kahn CR: Phosphatidylinositol 3-kinase activation is required for insulin stimulation of pp70 56 kinase, DNA synthesis, and glucose transporter translocation, Mol Cell Biol 1994, 14:4902-4911

47. Chung J, Grammer TC, Lemon KP, Kazlauskas A, Blenis J: PDGF- and insulindependent pp70S6 $\mathrm{k}$ activation mediated by phosphatidylinositol-3-OH kinase, Nature 1994, 370:71-75

48. von Manteuffel SR, Gingras AC, Ming XF, Sonenberg N, Thomas G: 4E-BP1 phosphorylation is mediated by the FRAP-p70s6k pathway and is independent of mitogen-activated protein kinase, Proc Natl Acad Sci U S A $1996,93: 4076-4080$

49. Gingras AC, Kennedy SG, O'Leary MA, Sonenberg N, Hay N: 4E-BP1, a repressor of mRNA translation, is phosphorylated and inactivated by the Akt(PKB) signaling pathway, Genes Dev 1998, 12:502-513

50. Pan D, Dong J, Zhang $Y$, Gao $X$ : Tuberous sclerosis complex: from Drosophila to human disease, Trends Cell Biol 2004, 14:78-85

51. Gao X, Pan D: TSC1 and TSC2 tumor suppressors antagonize insulin signaling in cell growth, Genes Dev 2001, 15:1383-1392

52. Potter $\mathrm{C}$, Huang $\mathrm{H}, \mathrm{Xu} \mathrm{T}$ : Drosophila Tsc1 functions with $\mathrm{Tsc} 2$ to antagonize insulin signaling in regulating cell growth, cell proliferation, and organ size, Cell 2001, 105:357-368

53. Tapon N, Ito N, Dickson BJ, Treisman JE, Hariharan IK: The Drosophila tuberous sclerosis complex gene homologs restrict cell growth and cell proliferation, Cell 2001, 105:345-355 


\section{Chapter 1}

54. Gao X, Zhang Y, Arrazola P, Hino O, Kobayashi T, Yeung RS, Ru B, Pan D: Tsc tumour suppressor proteins antagonize amino-acid-TOR signalling, Nat Cell Biol 2002, 4:699-704

55. Inokj K, Li Y, Zhu T, Wu J, Guan KL: TSC2 is phosphorylated and inhibited by Akt and suppresses mTOR signalling, Nat Cell Biol 2002, 4:648-657

56. Jaeschke A, Hartkamp J, Saitoh M, Roworth W, Nobukuni T, Hodges A, Sampson J, Thomas G, Lamb R: Tuberous sclerosis complex tumor suppressor-mediated $S 6$ kinase inhibition by phosphatidylinositide-3-OH kinase is mTOR independent, ] Cell Biol 2002, 159:217-224

57. Kwiatkowski DJ, Zhang $H$, Bandura JL, Heiberger KM, Glogauer $M$, elHashemite $N$, Onda $H$ : A mouse model of TSC1 reveals sex-dependent lethality from liver hemangiomas, and up-regulation of p70S6 kinase activity in Tsc1 null cells, Hum Mol Genet 2002, 11:525-534

58. Zhang Y, Gao X, Saucedo D, Ru B, Edgar BA, Pan D: Rheb is a direct target of the tuberous sclerosis tumour suppressor proteins, Nat Cell Biol 2003, 5:578-581

59. Dennis PB, Jaeschke A, Saitoh M, Fowler B, Kozma SC, Thomas G: Mammalian TOR: a homeostatic ATP sensor, Science 2001, 294:1102-1105

60. Carling D: The AMP-activated protein kinase cascade--a unifying system for energy control, Trends Biochem Sci 2004, 29:18-24

61. Inoki $\mathrm{K}$, Zhu T, Guan KL: TSC2 mediates cellular energy response to control cell growth and survival, Cell 2003, 115:577-590

62. Corradetti MN, Inoki K, Bardeesy N, DePinho RA, Guan KL: Regulation of the TSC pathway by LKB1: evidence of a molecular link between tuberous sclerosis complex and Peutz-Jeghers syndrome, Genes Dev 2004, 18:15331538

63. Shaw RJ, Bardeesy N, Manning BD, Lopez L, Kosmatka M, DePinho RA, Cantley LC: The LKB1 tumor suppressor negatively regulates mTOR signaling, Cancer Cell 2004, 6:91-99

64. Clemens MJ, Bommer UA: Translational control: the cancer connection, Int J Biochem Cell Biol 1999, 31:1-23

65. Zimmer SG, DeBenedetti A, Graff JR: Translational control of malignancy: the mRNA cap-binding protein, eIF-4E, as a central regulator of tumor formation, growth, invasion and metastasis, Anticancer Res 2000, 20:13431351

66. Li J, Yen C, Liaw D, Podsypanina K, Bose S, Wang SI, Puc J, Miliaresis C, Rodgers L, McCombie R, Bigner SH, Giovanella BC, Ittmann M, Tycko $B$ Hibshoosh $H$, Wigler $M H$, Parsons R: PTEN, a putative protein tyrosine phosphatase gene mutated in human brain, breast, and prostate cancer, Science 1997, 275:1943-1947

67. Steck PA, Pershouse MA, Jasser SA, Yung WK, Lin $H$, Ligon AH, Langford LA, Baumgard ML, Hattier T, Davis $T$, Frye $C$, Hu $R$, Swedlund $B$, Teng $D H$, Tavtigian SV: Identification of a candidate tumour suppressor gene, MMAC1, at chromosome 10q23.3 that is mutated in multiple advanced cancers, Nat Genet 1997, 15:356-362

68. Laughner E, Taghavi P, Chiles K, Mahon PC, Semenza GL: HER2 (neu) signaling increases the rate of hypoxia-inducible factor 1alpha (HIF-1alpha) synthesis: novel mechanism for HIF-1-mediated vascular endothelial growth factor expression, Mol Cell Biol 2001, 21:3995-4004

69. Young J, Povey S: The genetic basis of tuberous sclerosis, Mol Med Today $1998,4: 313-319$

70. Boudeau J, Sapkota G, Alessi DR: LKB1, a protein kinase regulating cell proliferation and polarity, FEBS Lett 2003, 546:159-165 
71. Kwiatkowski D]: Tuberous sclerosis: from tubers to mTOR, Ann Hum Genet 2003, 67:87-96

72. Lazaris-Karatzas A, Montine KS, Sonenberg N: Malignant transformation by a eukaryotic initiation factor subunit that binds to mRNA $5^{\prime}$ cap, Nature 1990, 345:544-547

73. Ruggero D, Montanaro L, Ma L, Xu W, Londei P, Cordon-Cardo C, Pandolfi PP: The translation factor eIF-4E promotes tumor formation and cooperates with c-Myc in lymphomagenesis, Nat Med 2004, 10:484-486

74. Wendel HG, De Stanchina E, Fridman JS, Malina A, Ray S, Kogan S, CordonCardo C, Pelletier J, Lowe SW: Survival signalling by Akt and eIF4E in oncogenesis and cancer therapy, Nature 2004, 428:332-337

75. Dever TE: Translation initiation: adept at adapting, Trends Biochem Sci 1999, 24:398-403

76. Kimball SR: Eukaryotic initiation factor elF2, Int J Biochem Cell Biol 1999, $31: 25-29$

77. Berry MJ, Knutson GS, Lasky SR, Munemitsu SM, Samuel CE: Mechanism of interferon action. Purification and substrate specificities of the doublestranded RNA-dependent protein kinase from untreated and interferontreated mouse fibroblasts, J Biol Chem 1985, 260:11240-11247

78. Chen JJ, London IM: Regulation of protein synthesis by heme-regulated eIF2 alpha kinase, Trends Biochem Sci 1995, 20:105-108

79. Cherkasova VA, Hinnebusch AG: Translational control by TOR and TAP42 through dephosphorylation of eIF2alpha kinase GCN2, Genes Dev 2003, 17:859-872

80. Harding HP, Zhang $Y$, Ron D: Protein translation and folding are coupled by an endoplasmic-reticulum-resident kinase, Nature 1999, 397:271-274

81. Harding HP, Zhang $Y$, Bertolotti A, Zeng $H$, Ron D: Perk is essential for translational regulation and cell survival during the unfolded protein response, Mol Cell 2000, 5:897-904

82. Bertolotti A, Zhang $Y$, Hendershot LM, Harding HP, Ron D: Dynamic interaction of BiP and ER stress transducers in the unfolded- protein response, Nat Cell Biol 2000, 2:326-332

83. Mathews $M$ B, Sonenberg N, W. HJ: Translational control of gene expression., Cold Spring Harbor: Cold Spring Harbor Laboratory. 2000, 1-31

84. Cardenas-Navia LI, Yu D, Braun RD, Brizel DM, Secomb TW, Dewhirst MW: Tumor-dependent Kinetics of Partial Pressure of Oxygen Fluctuations during Air and Oxygen Breathing, Cancer Res 2004, 64:6010-6017

85. Blais JD, Filipenko $V$, Bi M, Harding HP, Ron D, Koumenis C, Wouters BG, Bell JC: Activating transcription factor 4 is translationally regulated by hypoxic stress, Mol Cell Biol 2004, 24:7469-7482

86. Burda J, Martin ME, Gottlieb M, Chavko M, Marsala J, Alcazar A, Pavon M, Fando $J L$, Salinas $M$ : The intraischemic and early reperfusion changes of protein synthesis in the rat brain. eIF-2 alpha kinase activity and role of initiation factors eIF-2 alpha and eIF-4E, J Cereb Blood Flow Metab 1998, 18:59-66

87. Goldstein EN, Owen CR, White $B C$, Rafols JA: Ultrastructural localization of phosphorylated eIf2alpha [eIF2alpha(P)] in rat dorsal hippocampus during reperfusion, Acta Neuropathol (Berl) 1999, 98:493-505

88. Hu BR, Wieloch T: Stress-induced inhibition of protein synthesis initiation: modulation of initiation factor 2 and guanine nucleotide exchange factor activities following transient cerebral ischemia in the rat, J Neurosci 1993, $13: 1830-1838$ 
89. Paschen W: Disturbances of calcium homeostasis within the endoplasmic reticulum may contribute to the development of ischemic-cell damage, Med Hypotheses 1996, 47:283-288

90. Hahn GM: The heat shock response: events before, during, and after gene activation. Edited by Gautherie M. Heidelberg, Springer-Verlag, 1990, p. pp. 135-166

91. Jolly C, Morimoto RI: Role of the Heat Shock Response and Molecular Chaperones in Oncogenesis and Cell Death, J Natl Cancer Inst 2000, 92:1564-1572

92. Mathew A, Morimoto RI: Role of the heat-shock response in the life and death of proteins, Ann N Y Acad Sci 1998, 851:99-111

93. Morimoto RI: Regulation of the heat shock transcriptional response: cross talk between a family of heat shock factors, molecular chaperones, and negative regulators, Genes Dev 1998, 12:3788-3796

94. Benjamin IJ, Kroger B, Williams RS: Activation of the heat shock transcription factor by hypoxia in mammalian cells. Edited by $1990, \mathrm{p} . \mathrm{pp}$. 6263-6267

95. Giaccia AJ, Auger EA, Koong A, Terris DJ, Minchinton AI, Hahn GM, Brown JM: Activation of the heat shock transcription factor by hypoxia in normal and tumor cell lines in vivo and in vitro, Int. J. Radiat. Onc. Biol. Phys. 1992, 23:891-897

96. Patel B, Khaliq A, Jarvis-Evans J, Boulton M, Arrol S, Mackness M, McLeod D: Hypoxia induces HSP 70 gene expression in human hepatoma (HEP G2) cells, Biochem Mol Biol Int 1995, 36:907-912

97. Price BD, Mannheim-Rodman LA, Calderwood SK: Brefeldin A, thapsigargin, and AIF4- stimulate the accumulation of GRP78 mRNA in a cycloheximide dependent manner, whilst induction by hypoxia is independent of protein synthesis, J Cell Physiol 1992, 152:545-552

98. Song MS, Park YK, Lee JH, Park K: Induction of glucose-regulated protein 78 by chronic hypoxia in human gastric tumor cells through a protein kinase Cepsilon/ERK/AP-1 signaling cascade, Cancer Res 2001, 61:8322-8330.

99. Koong $A C$, Auger EA, Chen EY, Giaccia AJ: The regulation of GRP78 and messenger RNA levels by hypoxia is modulated by protein kinase $C$ activators and inhibitors, Radiat Res 1994, 138:560-63

100. Koong AC, Chen EY, Lee AS, Brown JM, Giaccia AJ: Increased cytotoxicity of chronic hypoxic cells by molecular inhibition of GRP78 induction, Int J Radiat Oncol Biol Phys 1994, 28:661-666

101. Harding HP, Zhang $Y$, Zeng $H$, Novoa I, Lu PD, Calfon M, Sadri N, Yun C, Popko B, Paules R, Stojdl DF, Bell JC, Hettmann T, Leiden JM, Ron D: An integrated stress response regulates amino acid metabolism and resistance to oxidative stress, Mol Cell 2003, 11:619-633

102. Liu CY, Kaufman RJ: The unfolded protein response, J Cell Sci 2003, 116:1861-1862

103. Urano $F$, Wang $X$, Bertolotti $A$, Zhang $Y$, Chung $P$, Harding HP, Ron D: Coupling of stress in the ER to activation of JNK protein kinases by transmembrane protein kinase IRE1, Science 2000, 287:664-666

104. Ron D: Translational control in the endoplasmic reticulum stress response, J Clin Invest 2002, 110:1383-1388

105. Harding $\mathrm{HP}$, Calfon M, Urano $F$, Novoa I, Ron D: Transcriptional and translational control in the Mammalian unfolded protein response, Annu Rev Cell Dev Biol 2002, 18:575-599 
106. Ameri $K$, Lewis $C E$, Raida M, Sowter $H$, Hai T, Harris AL: Anoxic induction of ATF-4 through HIF-1-independent pathways of protein stabilization in human cancer cells, Blood 2004, 103:1876-1882

107. Romero-Ramirez L, Cao $H$, Nelson D, Hammond $E$, Lee A-H, Yoshida $H$, Mori K, Glimcher LH, Denko NC, Giaccia AJ, Le Q-T, Koong AC: XBP1 Is Essential for Survival under Hypoxic Conditions and Is Required for Tumor Growth, Cancer Res 2004, 64:5943-5947

108. Scheuner D, Song B, McEwen E, Liu C, Laybutt R, Gillespie P, Saunders T, Bonner-Weir $S$, Kaufman RJ: Translational control is required for the unfolded protein response and in vivo glucose homeostasis, Mol Cell 2001, 7:1165-1176. 


\section{CHAPTER 2}

Gene expression during acute and prolonged hypoxia is regulated by distinct mechanisms of translational control

EMBO J. 2006 Feb 9

Koritzinsky $M$, Magagnin $M G^{*}$, van den Beucken $T^{*}$,

Seigneuric R, Savelkouls K, Dostie J, Pyronnet S, Kaufman RJ, Weppler SA, Voncken JW, Lambin P, Koumenis C, Sonenberg N, Wouters BG.

*These two authors contributed equally 


\title{
Chapter 2
}

\begin{abstract}
Hypoxia has recently been shown to activate the endoplasmic reticulum kinase PERK, leading to phosphorylation of eIF2 $\alpha$ and inhibition of MRNA translation initiation. Using a quantitative assay, we show that this inhibition exhibits a biphasic response mediated through two distinct pathways. The first occurs rapidly, reaching a maximum at $1-2 \mathrm{~h}$ and is due to phosphorylation of eIF $2 \alpha$. Continued hypoxic exposure activates a second, eIF $2 \alpha$ independent pathway that maintains repression of translation. This phase is characterized by disruption of eIF4F and sequestration of eIF4E by its inhibitor 4E-BP1 and transporter 4E-T. Quantitative RT-PCR analysis of polysomal RNA indicates that the translation efficiency of individual genes varies widely during hypoxia. Furthermore, the translation efficiency of individual genes is dynamic, changing dramatically during hypoxic exposure due to the initial phosphorylation and subsequent dephosphorylation of eIF2 $\alpha$. Together, our data indicate that acute and prolonged hypoxia regulates mRNA translation through distinct mechanisms, each with important contributions to hypoxic gene expression.
\end{abstract}




\section{Introduction}

The presence of hypoxic and anoxic areas in human tumors is well documented, and is prognostic for poor outcome (reviewed $\operatorname{in}^{1,2}$. The clinical importance of tumor hypoxia esults from its ability to protect cells against both radiation and chemotherapy and from the fact that it can provide a selection pressure for apoptotically resistant cells ${ }^{3}$. Furthermore, the cellular response to hypoxia causes important changes in gene expression that affect cell behavior and influence patient prognosis. There has been particular focus on changes mediated through the family of hypoxia-inducible transcription factors (HIFs). HIF-1 and HIF-2 promote transcription of more than 60 putative downstream genes (for a review $\mathrm{see}^{4}$ ) that affect hypoxia tolerance, energy homeostasis, angiogenesis and tumor growth. Although the transcriptional response to hypoxia is clearly very important ${ }^{5-7}$, tumor cells also experience short, transient exposures to hypoxia and/or anoxia that occur over time frames too fast for an effective transcriptional response. Transient changes in oxygenation occur owing to the abnormal vasculature found in most tumors, characterized by immature, leaky and improperly formed vessels. Perfusion of these vessels can change dynamically in time, leading to rapid but transient episodes of severe hypoxia in the tumor cells dependent upon them ${ }^{8,9}$. Consequently, post-transcriptional responses are presumably important for adaptation to cycling oxygenation in tumors.

Control of mRNA translation during hypoxia is emerging as an important cellular response to hypoxia ${ }^{10-12}$. As protein synthesis is energy costly, inhibition of mRNA translation may represent an active response to prevent loss of energy homeostasis during hypoxia. Indeed, it has been shown that overall mRNA translation is severely but reversibly inhibited during hypoxia ${ }^{10}, 13,14$ with kinetics that precede ATP depletion ${ }^{15}$. Furthermore, regulation of mRNA translation can have a significant and rapid impact on individual gene expression. This is because the sensitivity of individual genes to changes in overall translation varies widely and in a 
manner that reflects the molecular mechanisms responsible for controlling translation ${ }^{16,17}$. Regulation of gene expression through control of mRNA translation is important during various pathologies including cancer ${ }^{18}$. The mechanisms responsible for inhibiting translation during hypoxia are not yet fully understood.

We have previously investigated the involvement of the endoplasmic reticulum (ER) kinase PERK in the hypoxia induced downregulation of protein synthesis ${ }^{10}$. PERK is activated as part of the evolutionarily conserved unfolded protein response (UPR) (reviewed $\mathrm{in}^{19}$. It phosphorylates eIF2 $\alpha$, a subunit of eIF2, which in its GTP-bound form recruits the aminoacylated tRNA to the 405 ribosomal subunit. The exchange of GDP for GTP is mediated by the guanine nucleotide exchange factor eIF2B. Ser51-phosphorylated eIF2 $\alpha$ inhibits eIF2B, resulting in inhibition of translation initiation. eIF $2 \alpha$ phosphorylation results in a set of molecular events collectively termed the integrated stress response. These include the inhibition of global mRNA translation in conjunction with induced expression of the transcription factor ATF4 and its downstream target genes ${ }^{20}$. We showed that hypoxia rapidly activated PERK, which led to reversible phosphorylation of eIF $2 \alpha^{10}$. Hypoxia-induced inhibition of protein synthesis was severely attenuated in cells without functional PERK. After prolonged periods of hypoxia, PERK-deficient cells did show partial inhibition, suggesting that protein synthesis is regulated through additional mechanisms.

Another candidate mechanism for inhibiting translation during hypoxia is disruption of the cap-binding protein complex eIF4F, which consists of eIF4E, eIF4A and eIF4G (for recent reviews $s^{21,22}$. eIF4E participates in a protein bridge between the mRNA and the ribosome by its simultaneous interaction with the mRNA 50 cap structure and the large scaffolding protein eIF4G, which in turn interacts with eIF3 that is bound to the $40 \mathrm{~S}$ ribosomal subunit. eIF4E is regulated through a set of binding proteins (4E-BPs) that bind reversibly to eIF4E in their hypophosphorylated form, and this obstructs the interaction between eIF4E and eIF4G. The 4EBP1 
protein becomes hyperphosphorylated in response to a number of stimuli, such as insulin, hormones, growth factors, mitogens and cytokines, as a result of activation of the PI3-kinase/Akt/FRAPmTOR pathway ${ }^{23}$.

It remains unclear to what degree the lack of eIF4F assembly contributes to inhibition of translation during tumor hypoxia. Several studies have investigated the combined consequences of ischemia/reperfusion on eIF4F-related proteins in rat brains (reviewed $\mathrm{in}^{24,25}$. Proteolysis of eIF4G was reported during ischemia and reperfusion in vivo ${ }^{26,27}$, but not in neuronal cells cultured in vitro (NGF differentiated PC12 cells) ${ }^{28}$. The reports addressing the expression and phosphorylation status of eIF4E during ischemia are conflicting, but 4E-BP1 dephosphorylation has been demonstrated both in vivo and in vitro ${ }^{27,} 28$. The acuteness and complexity of ischemia/reperfusion stress and the high sensitivity of neurons to deprivation and reconstitution of both oxygen and nutrients are distinct properties of this model system and thus difficult to extrapolate to tumor hypoxia. In rat hepatocytes, 4E-BP1 becomes dephosphorylated and associates with eIF4E rapidly (15-60 $\mathrm{min}$ ) upon mild hypoxia, but this could not explain the observed downregulation of protein synthesis ${ }^{29}$. More recently, it was reported that hypoxia could influence 4E-BP1 phosphorylation by affecting the activity of mTOR ${ }^{30}$. Serum-starved and hypoxic human embryonic kidney cells failed to activate mTOR, phosphorylate 4E-BP1 and dissociate 4E-BP1 from eIF4E in response to insulin treatment. Nonetheless, it remains unknown whether hypoxia alone is sufficient to disrupt the eIF4F complex and to what extent this influences overall translation during hypoxia. Here we show that hypoxia induces a biphasic inhibition of mRNA translation characterized by transient phosphorylation of eIF2 $\alpha$ and subsequent dissociation of eIF4F. These two mechanisms operate independently of each other and both have important consequences for gene expression during hypoxia. 


\section{Materials and methods}

\section{Cell culture}

Exponentially growing cervical carcinoma HeLa cells (American Type Culture Collection CCL-2), lung adenocarcinoma A549 cells, normal human fibroblasts (AG1522) or MEFs that were WT or had a homozygous knock-in mutation for eIF2 $\alpha$ (S51A) (Scheuner et al, 2001) were grown on glass dishes or chamber slides in DMEM media supplemented with $10 \%$ fetal calf serum. The MEF media also contained MEM nonessential amino acids and $55 \mathrm{mM}$ 2-mercaptoethanol (all Sigma-Aldrich). For preparation of extracts and viability assessments, see Supplementary data.

\section{Hypoxic conditions}

Cells were transferred to a hypoxic culture chamber (MACS VA500 microaerophilic workstation, Don Whitley Scientific). The composition of the atmosphere in the chamber consisted of $5 \% \mathrm{H}_{2}, 5 \% \mathrm{CO}_{2}, 0.0 \% \mathrm{O}_{2}$ and residual $\mathrm{N}_{2}$.

\section{M7GTP resin precipitation}

A $1 \mathrm{mg}$ portion of HeLa extract was incubated with $25 \mathrm{ml}$ of $\mathrm{m7GTP}$ sepharose resin (Amersham Biosciences) for $3 \mathrm{~h}$ at $4^{\circ} \mathrm{C}$. The resin was washed, boiled in Laemmli buffer and the polypeptides were resolved by SDS-PAGE.

\section{Western blotting}

Cell extracts were boiled in Laemmli buffer and polypeptides were resolved by SDS-PAGE and transferred onto $0.2 \mathrm{~mm}$ nitrocellulose

membranes (Amersham Corp.). For primary antibodies, see Supplementary data. Detection of peroxidase-coupled secondary antibodies was performed with Enhanced Chemiluminescence (Amersham Corp.). 


\section{Gene-specific translation during hypoxid}

\section{Immunofluorescence}

Cells were fixed with $4 \%$ paraformaldehyde and permeabilized in $4 \%$ paraformaldehyde and $0.1 \%$ Triton $X-100$. For antibodies, see Supplementary data. Cells were mounted in the ProLongTM Antifade Kit (Molecular Probes) and analyzed with a Zeiss inverted LSM 410 laser scan confocal microscope.

\section{Polysomal fractionation and analysis}

Polysomal fractionation and analysis were performed as described previously (Koritzinsky et al, 2005); see Supplementary data.

\section{RNA isolation and reverse transcription}

RNA isolation and reverse transcription were performed as described previously (Koritzinsky et al, 2005); see Supplementary data.

\section{Quantitative PCR analysis}

Real-time PCR was performed in either ABI 7700 or ABI 7500 (Applied Biosystems). For primers and probes, see Supplementary data. Unfractionated samples were normalized by $18 S$ rRNA signal. Samples from polysome fractions were normalized by $18 \mathrm{~S}$ rRNA measured by PCR divided by $18 \mathrm{~S}$ rRNA measured by spectrometry during fractionation, corrected for loading. This facilitated correction for any differences in RNA isolation or reverse transcriptase efficiency between samples. The abundance of every gene was calculated relative to a master reference using standard curves.

\section{Supplementary data}

Supplementary data are available at The EMBO Journal Online. 


\section{Results}

\section{Kinetics of translation inhibition}

To determine the effects of hypoxia on mRNA translation initiation in HeLa cells, we examined the association of ribosomes with MRNA at various time points. In this assay, the number of ribosomes found within the 'polysomal' fraction of mRNA (mRNA containing two or more ribosomes) is a reflection of de novo protein synthesis. This technique is advantageous to other methods such as $35 \mathrm{~S}$ incorporation, which requires prior aminoacid starvation, a procedure that can itself influence translation initiation $^{31}$. Figure $1 \mathrm{~A}$ shows that at all time points examined, hypoxia causes a large decrease in polysomal mRNA and a corresponding increase in free ribosomes and ribosomal subunits. The reduction in translation is not influenced by cell death, as cell viability remains above $90 \%$ following $16 \mathrm{~h}$ of hypoxia (data not shown). Furthermore, the inhibition of translation is completely reversible upon reoxygenation (data not shown).

To assess quantitatively overall mRNA translation from the polysome profiles, we calculated the percentage of rRNA participating in polysomes and defined this as the overall translation efficiency. This value is reduced from 62 to $24 \%$ after $1 \mathrm{~h}$ of hypoxia, and then recovers somewhat stabilizing at $30 \%$ (Figure 1B). The drop in translation reproducibly exhibited this biphasic response with maximum inhibition after 1-2 $h$, followed by a small recovery. The magnitude of inhibition is comparable to that observed following complete disruption of the cellular redox environment with $1 \mathrm{mM}$ dithiothreitol (DTT) (17\%) (data not shown).

Analysis of the polysome profiles in Figure $1 \mathrm{~A}$ shows that hypoxia also causes a change in the distribution of the polysomal mRNA, with proportionally less signal in the higher molecular weight fractions. This indicates that the average number of ribosomes per mRNA transcript is also decreased during hypoxia, reflecting a reduction in translation initiation efficiency even for those transcripts that remain translated. From 
A
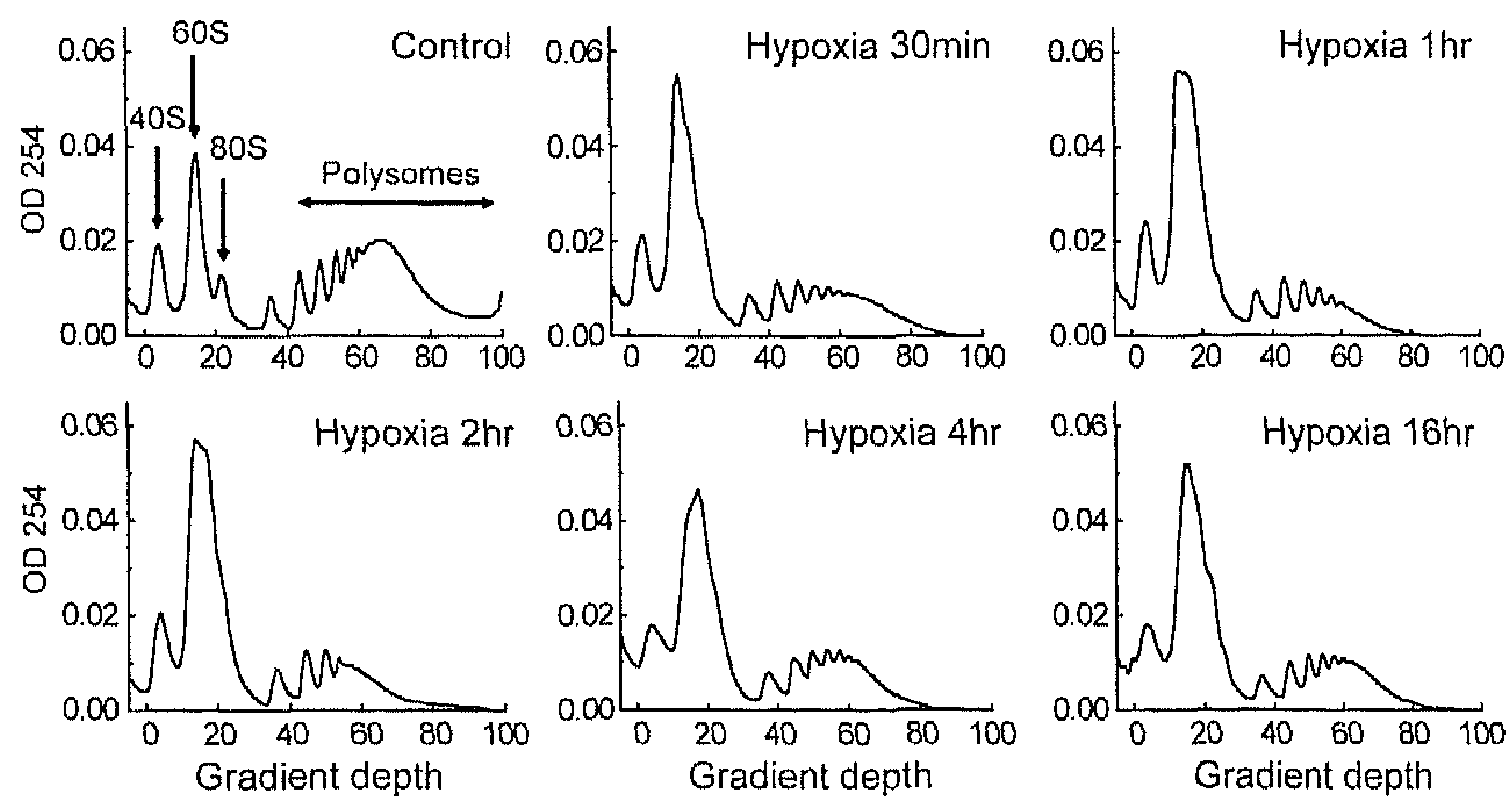

B

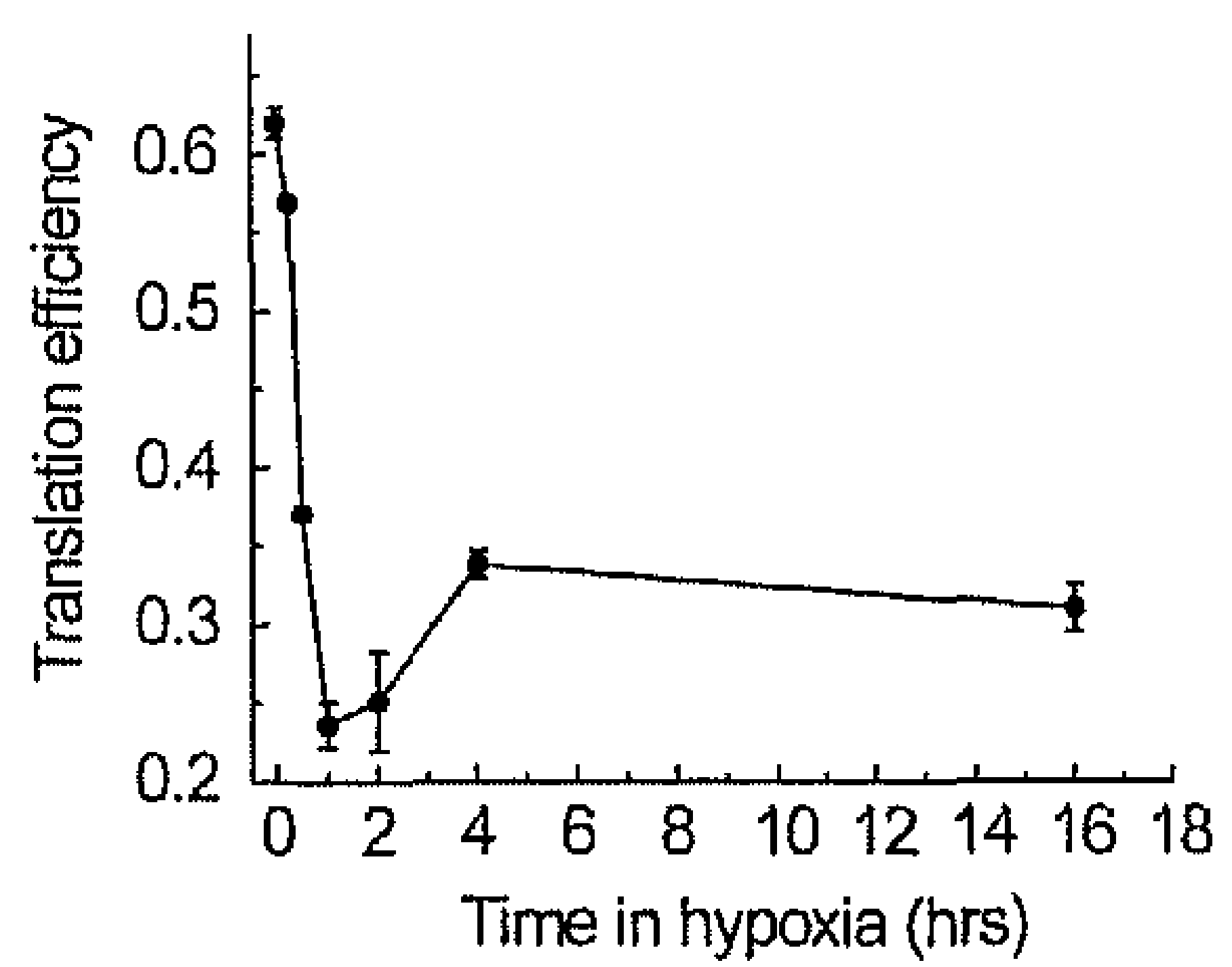

C

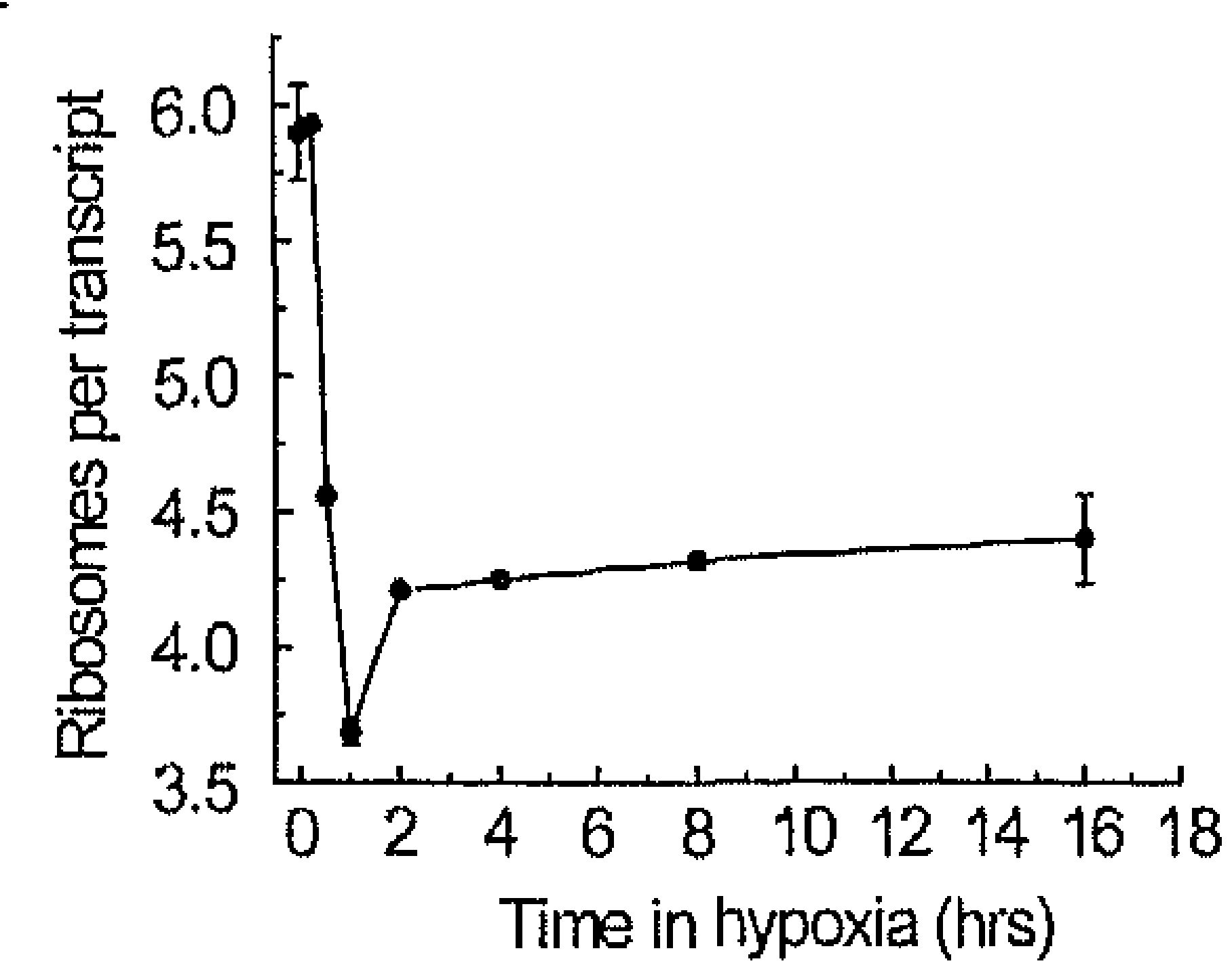

Figure 1. Hypoxia inhibits mRNA translation. HeLa cells were exposed to $0.0 \% \mathrm{O}_{2}$ for $0-16 \mathrm{~h}$ and cell lysates were separated on a sucrose gradient. (A) The optical density $(O D)$ at $254 \mathrm{~nm}$ is shown as a function of gradient depth for each time point. Actively translated mRNA is associated with high-molecular-weight polysomes deep in the gradient. (B) Translation efficiency in HeLa cells as a function of time in $0.0 \%$ $\mathrm{O}_{2}$. As a measure of overall translation efficiency, the relative amount of rRNA participating in polysomes was estimated. This fraction is proportional to the integrated area under the curve containing polysomes, as marked in (A). (C) The average number of ribosomes per mRNA in the polysomes as a function of time in $0.0 \% \mathrm{O}_{2}$. This was calculated by differential integration of the profiles in (A). 
the polysome profiles, we calculated the average number of ribosomes per translated transcript (i.e. mRNAs containing two or more ribosomes) at different time points during hypoxia (Figure 1C). The kinetics of this parameter follow in large part that of the overall translation.
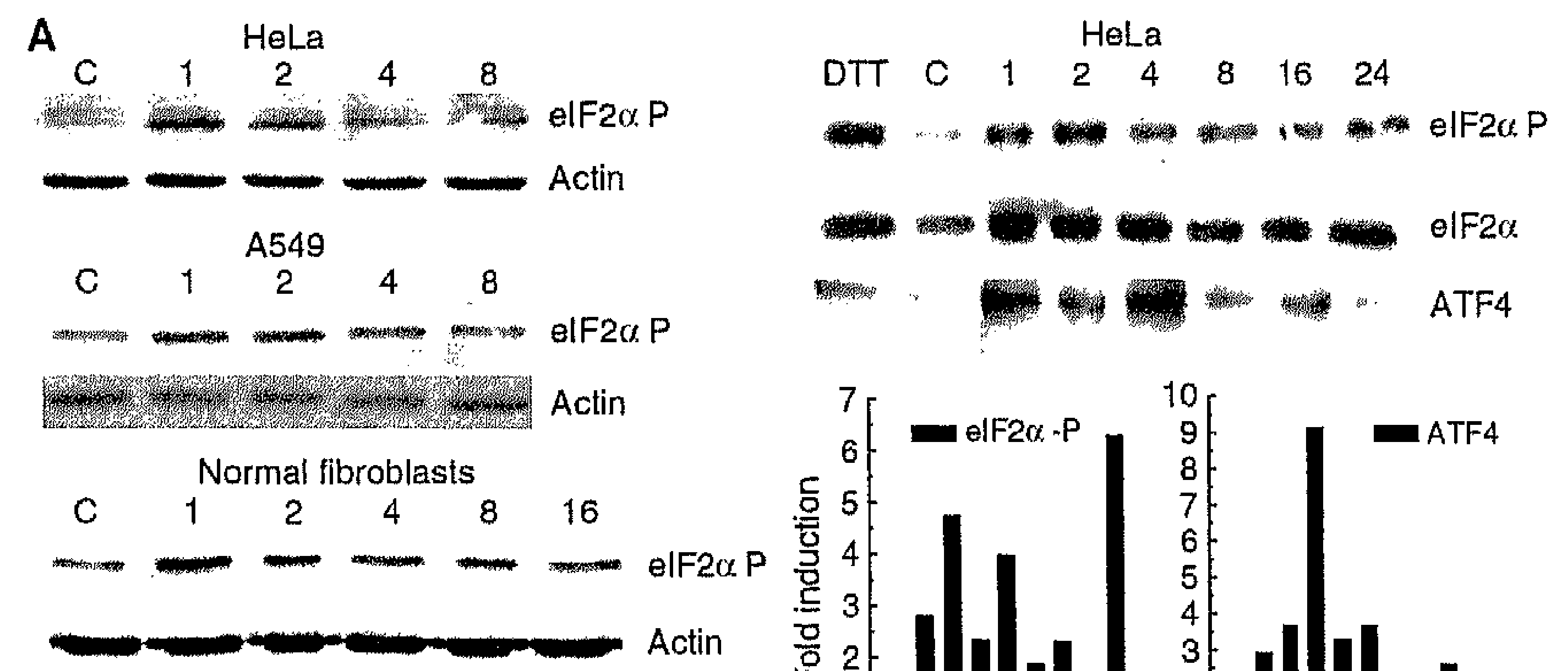

Actin
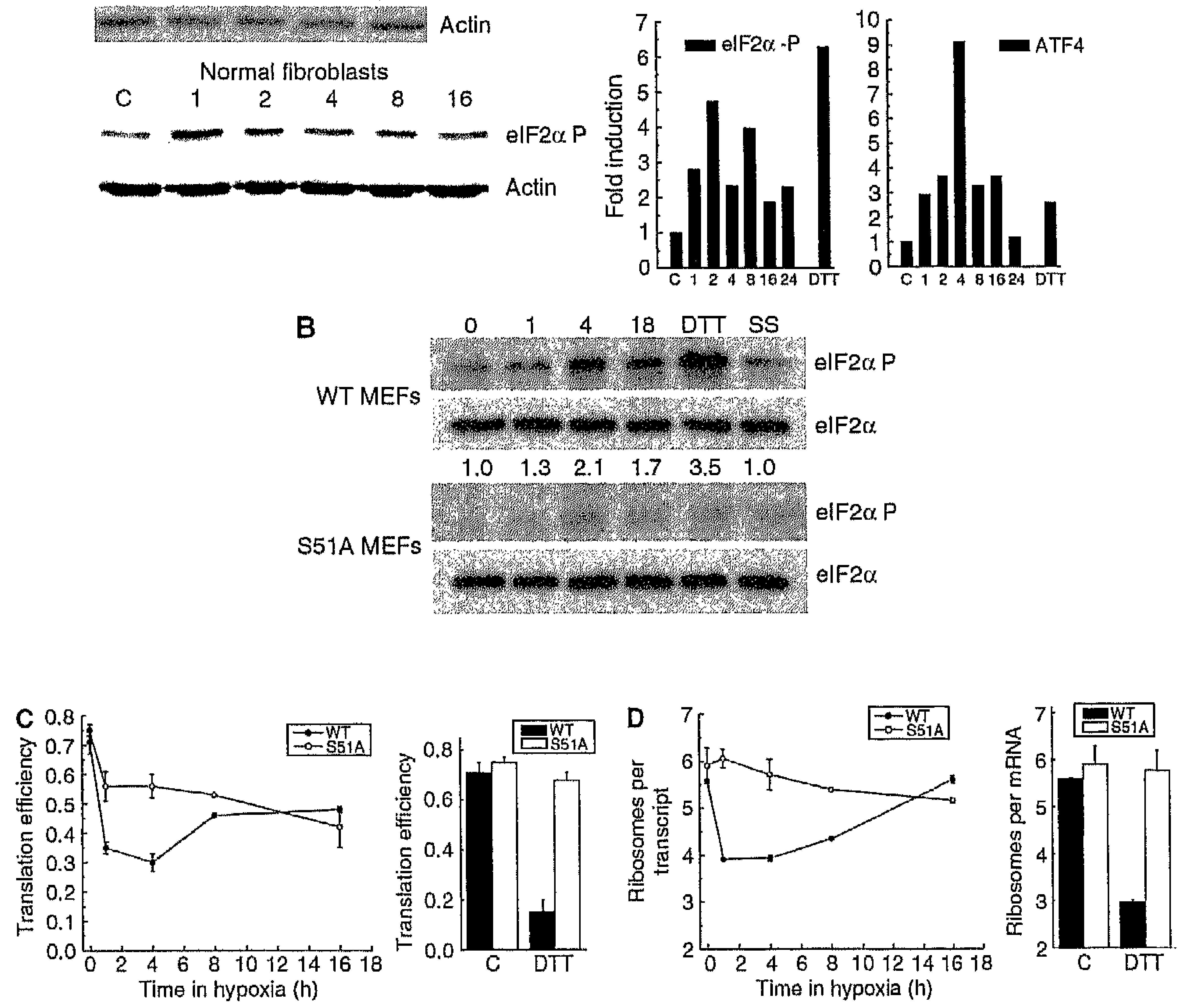


\section{eIF $2 \alpha$ regulates translation during acute hypoxia}

The eIF2 $\alpha$ kinase PERK is at least partly responsible for protein synthesis inhibition during acute hypoxia, as measured by radioactive labeling of newly synthesized proteins ${ }^{10}$. Thus, we hypothesized that the rapid inhibition and subsequent partial recovery in translation is due to changes in eIF $2 \alpha$ phosphorylation. Indeed, we found that the phosphorylation of eIF $2 \alpha$ is greatest after $1-2 h$ and then decreases by $8 h$ of hypoxia in several cell lines (Figure 2A). ATF4 protein levels also increase and then decrease during hypoxia in a manner that mirrors eIF $2 \alpha$ phosphorylation. The dynamics of eIF $2 \alpha$ phosphorylation and ATF4 protein induction thus correlate with the initial inhibition oftranslation and its subsequent recovery. To assess the requirement of eIF2 $\alpha$ phosphorylation for translation inhibition during hypoxia we examined the response of mouse embryo fibroblasts (MEFs) derived from eIF $2 \alpha$ knock-in mice containing an S51A mutation ${ }^{32}$. As expected, these cells were defective in phosphorylation of eIF2 $\alpha$ during hypoxia (Figure 2B). The translation efficiency in wild-type (WT) MEFs is similar to that in HeLa cells, with a rapid drop during acute hypoxia followed by a partial recovery (Figure 2C). In contrast, S51A MEFs display a substantial defect in their ability to inhibit translation during the initial phase.

Figure 2. Inhibition of translation during acute hypoxia is dependent on eIF2a. HeLa cells, A549 cells, human normal fibroblasts (NF) and WT or S51A MEFs were exposed to $0.0 \% \mathrm{O}_{2}$ for $0-16 \mathrm{~h}, 1 \mathrm{mM}$ DTT or serum starvation (SS) for $1 \mathrm{~h}$. Cell lysates were separated by SDS-PAGE. Immunoblots for (A) HeLa, A549 and NF or (B) MEFs were performed using antibodies against total or phosphorylated eIF2 $\alpha$, ATF4 and $\beta$-actin. In (A), optical densitometry for phosphorylated eIF $2 \alpha$ or ATF4 normalized by total eIF $2 \alpha$ is also shown. Total elF $2 \alpha$ expression has previously been shown to be constant during hypoxia ${ }^{10}$. (C) Cell lysates were separated on a sucrose gradient, and $O D$ at $254 \mathrm{~nm}$ was recorded. Translation efficiency as a function of time in $0.0 \% \mathrm{O}_{2}$ in WT and S51A MEFs was estimated as in Figure 1. (D) Average number of ribosomes per mRNA in the polysomes in WT and S51A MEFs as a function of time in $0.0 \% \mathrm{O}_{2}$ was calculated as in Figure 1. 


\section{Chaprer 2}

Nonetheless, after $16 \mathrm{~h}$ of hypoxia, both cell lines show a similar loss in translation efficiency. These data indicate that eIF $2 \alpha$ phosphorylation is indeed necessary for inhibition of translation during acute hypoxia, but not at later times. When the polysome profiles are analyzed in terms of the average number of ribosomes per translated transcript, S51A MEFs exhibit an even stronger defect in their response during acute hypoxia. Despite a small but detectable drop in translation efficiency during the first $4 \mathrm{~h}$ of hypoxia

(Figure 2C), S51A MEFs show no decrease in the average number of ribosomes per translated transcript (Figure 2D). The same result was found in cells treated with DTT, a known activator of PERK that causes eIF2 $\alpha$ phosphorylation. In contrast, WT MEFs show a strong reduction in average ribosomes per transcript during both acute hypoxia and DTT treatment. Interestingly, after $8 \mathrm{~h}$ of hypoxia, the average number of ribosomes per translated transcript increases again toward normal levels in WT cells and is equivalent to that in S51A MEFs by $16 \mathrm{~h}$. These data provide further evidence that the inhibition of translation that occurs after acute and prolonged hypoxia is mechanistically distinct.

\section{Disruption of the eIF4F complex during hypoxia}

The assembly of the cap-binding complex eIF4F is a common control point for translation initiation and was thus a likely candidate for maintaining low rates of translation during prolonged hypoxia. We examined the levels of eIF4E and proteins that associate with it as an active complex (eIF4GI) or as an inactive complex (4E-BP1). Figure $3 A$ shows that the levels of eIF4E do not change during hypoxia. In contrast, 4EBP1 (Figure 3B) shows both a small induction at $8 \mathrm{~h}$ and a strong dephosphorylation after $16 \mathrm{~h}$ of hypoxia. This protein runs as different migrating bands representing different phosphorylation levels. The fastest migrating band is substantially increased after $16 \mathrm{~h}$ of hypoxia, and represents the 
hypophosphorylated 4E-BP1, which is known to have a higher affinity for eIF4E. A small decrease in the abundance of the scaffold protein eIF4GI (Figure $3 \mathrm{C}$ ) was observed after $8 \mathrm{~h}$, consistent with a decrease in its rate of synthesis measured in a microarray study using polysomal RNA (unpublished data). Overexposure of the blots indicated no reproducible changes in the cleavage of eIF4G. The influence of hypoxia on 4E-BP1 phosphorylation appears to be largely independent of eIF2 $\alpha$ phosphorylation, as it is not differentially affected in the WT and S51A MEFs (unpublished data). However, until the relative contributions of various upstream signaling pathways to 4E-BP phosphorylation under hypoxia are better understood, it is premature to conclude that no connection between eIF $2 \alpha$ and eIF4F exists.

A

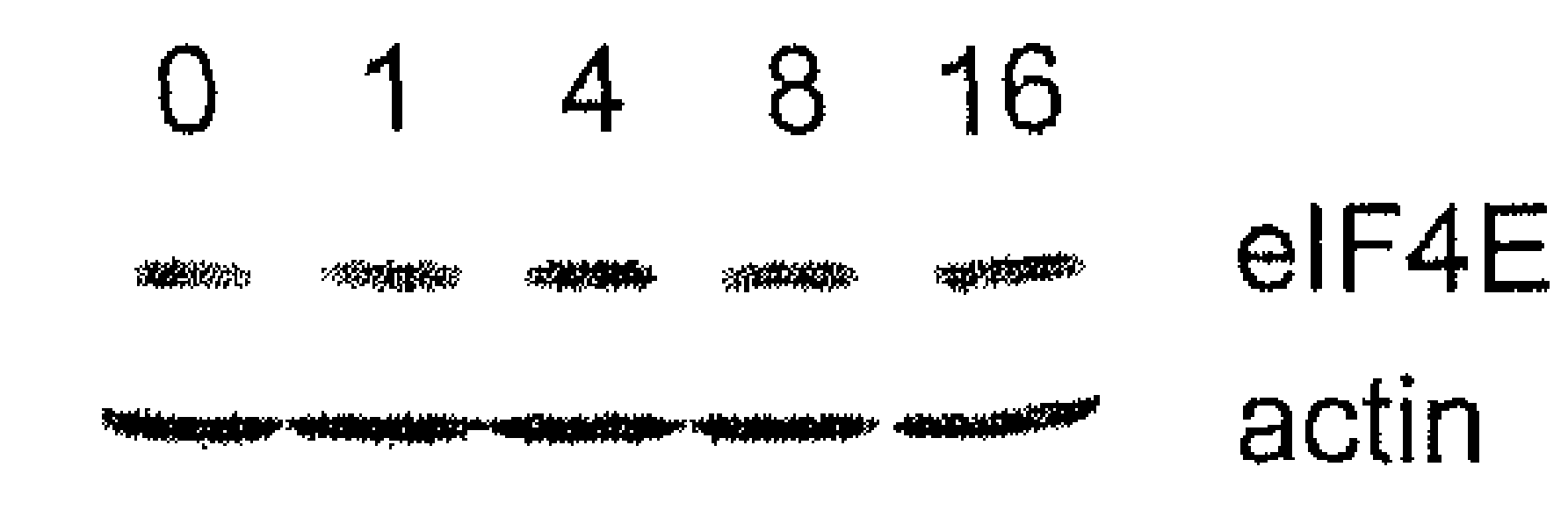

B

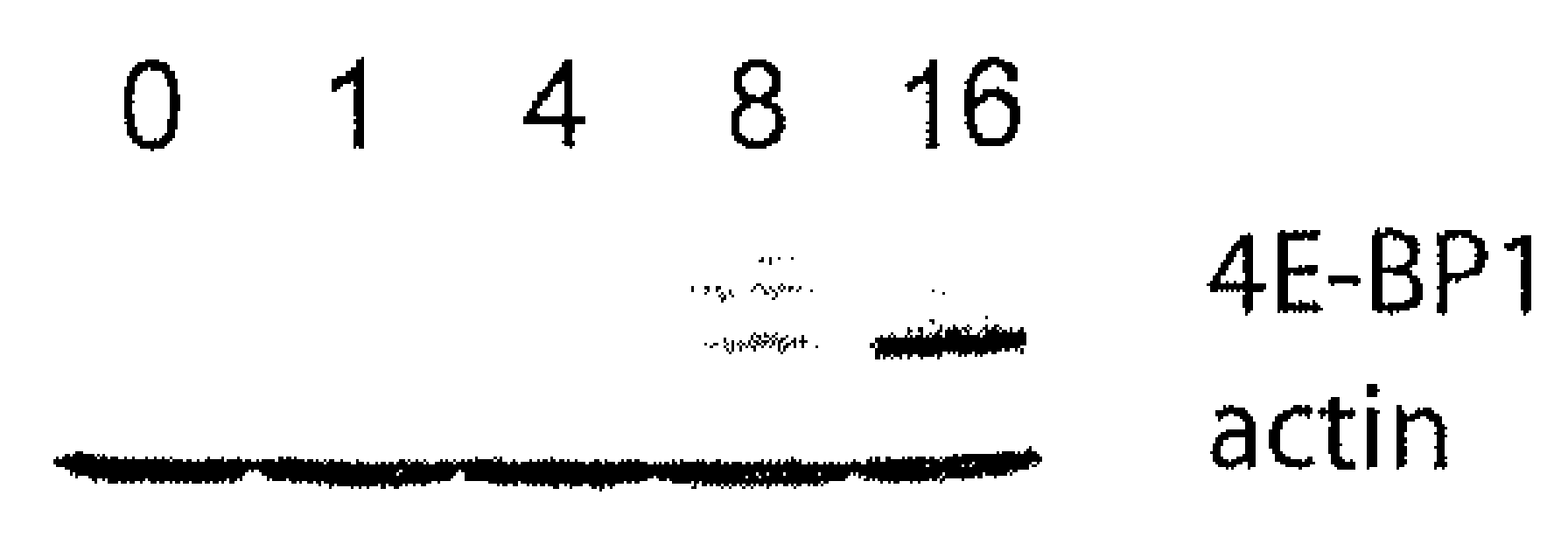

$\begin{array}{llllll}C & 0 & 1 & 4 & 8 & 16\end{array}$

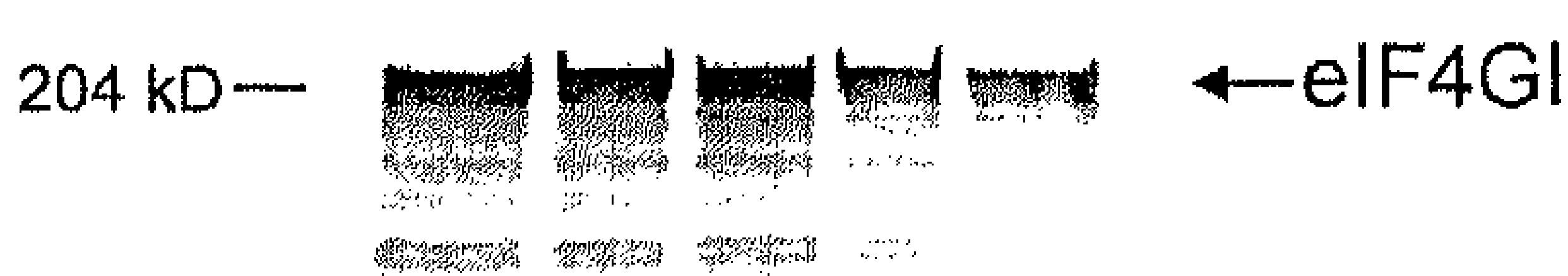

$123 \mathrm{kD}-$

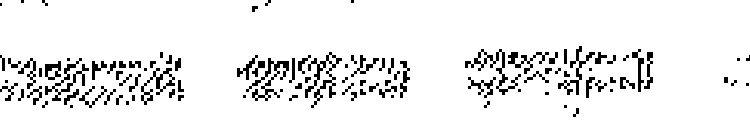

$80 \mathrm{kD}-$

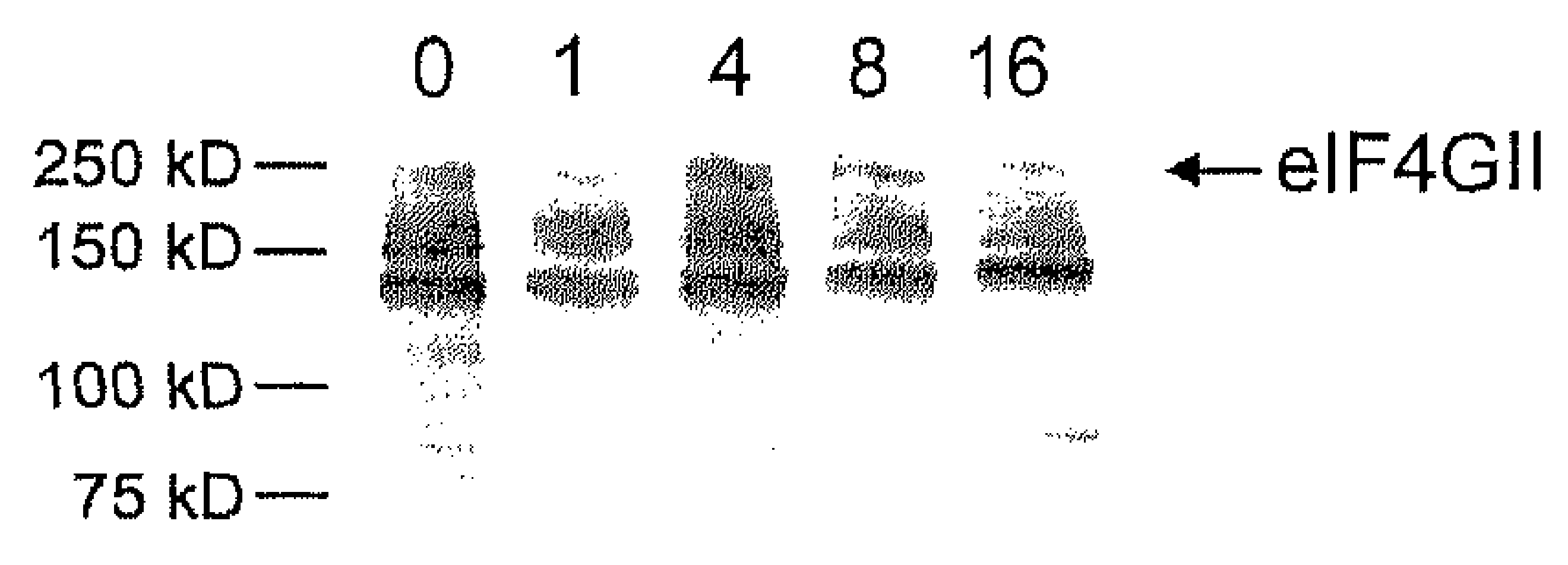

Figure 3. Expression of eIF4E, 4E-BP1 and eIF4GI during hypoxia. HeLa cells were exposed to $0.0 \% \mathrm{O}_{2}$ for $0-16 \mathrm{~h}$ and cell lysates separated by SDS-PAGE. Immunoblots were performed using antibodies against actin, (A) eIF4E, (B) 4E-BP1 and (C) eIF4GI. The phosphorylation forms of 4EBP1 have different electrophoretic mobilities and are represented by several bands on the immunoblot. Fulllength eIF4GI run at about 220 $\mathrm{kDa}$; the blot is overexposed to detect cleavage products. 
A

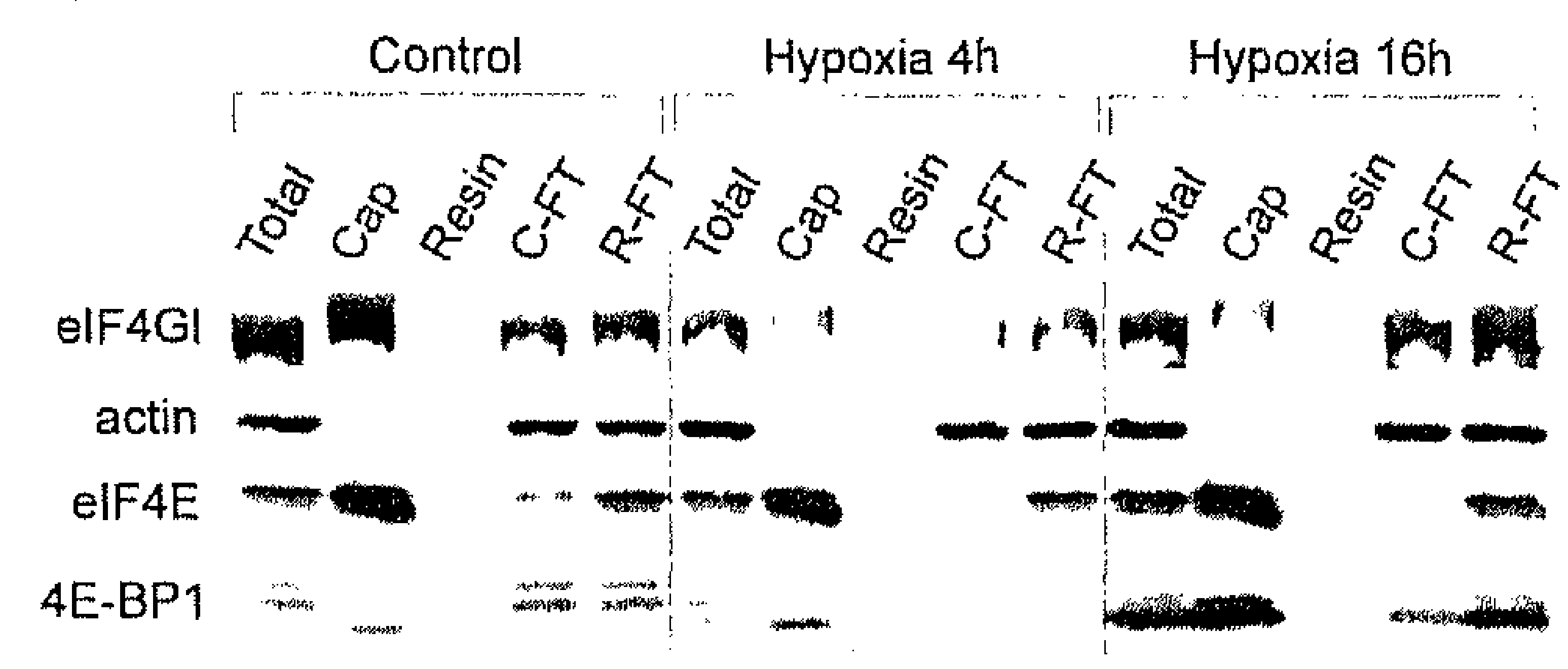

B

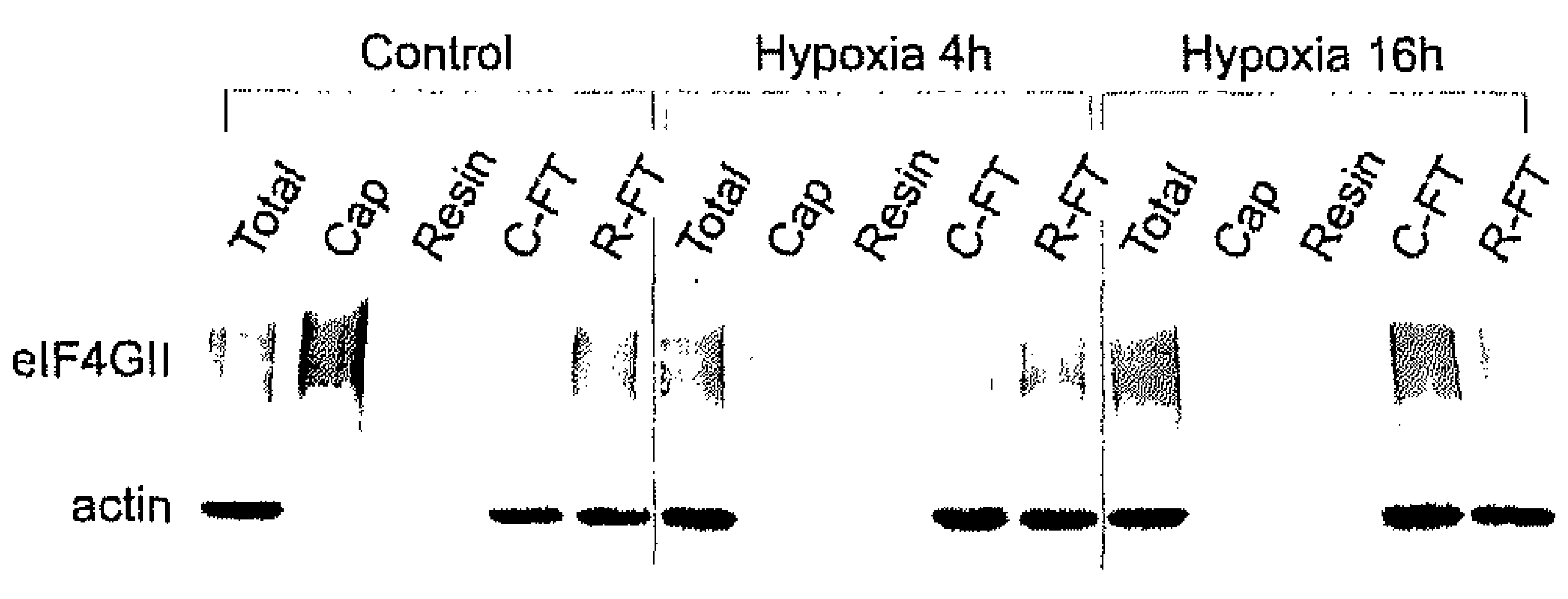

Figure 4. eIF4F is disrupted during prolonged hypoxia. HeLa cells were exposed to $0.0 \% \mathrm{O}_{2}$ for $0-16 \mathrm{~h}$ and cell lysates probed for the presence of various eIF4E complexes. Lysates were incubated with an m7-cap analogue ('Cap') or uncapped resin as a negative control. Immunoblots were performed with antibodies against actin, (A) eIF4GI, eIF4E, 4E-BP1 and (B) eIF4GII. 'Cap': proteins bound to the capped resin; 'Resin': proteins bound to the uncapped resin; 'C-FT': unbound fraction after incubation with capped resin; 'R-FT': unbound fraction after incubation with uncapped resin.

To more strictly assess the influence of hypoxia on eIF4F, we investigated the association of eIF4E with eIF4GI and eIF4GII as well as with its inhibitor 4E-BP1 in HeLa cells. During aerobic conditions where translation is efficient, eIF4E is associated with large amounts of both eIF4GI and eIF4GII, and only a small amount of 4E-BP1 (cap lanes in Figure 4A and B). Cap-associated eIF4G migrated somewhat slower than the overall pool of eIF4G, suggesting a possible modification of this phospho-protein when bound to the cap. In contrast, after 4 or $16 \mathrm{~h}$ of hypoxia, there is a dramatic loss in binding to both eIF4GI and eIF4GII, indicating dissociation of the eIF4F complex. At $16 \mathrm{~h}$, this dissociation correlates with 
a large increase in binding between eIF4E and 4E-BP1, consistent with the increase in the hypophosphorylated levels of $4 \mathrm{E}-\mathrm{BP} 1$ at this time. It also correlated with decreased phosphorylation of eIF4E (Supplementary Figure S1) at $16 \mathrm{~h}$, but the physiological significance of this remains unclear. However, although dissociation of eIF4G and eIF4E is complete after $4 \mathrm{~h}$ of hypoxia, a corresponding change in eIF4E phosphorylation or eIF4E/4E-BP1 association is not seen at this time point. This suggests that a mechanism distinct from 4E-BP1 dephosphorylation may also inhibit eIF4F during hypoxia.

\section{Translocation of eIF4E by $4 E-T$}

A potential cause of eIF4F disruption that has not been well characterized is the translocation of eIF4E to the nucleus or to cytoplasmic bodies of mRNA processing (P-bodies). A $5-20 \%$ fraction of eIF4E is known to localize to the cell nucleus ${ }^{33}$. The shuttling protein $4 \mathrm{E}-\mathrm{T}$ is the only known regulator of eIF4E localization and is capable of binding and transporting it to the cell nucleus ${ }^{34}$. eIF4E also colocalizes with 4E-T in P-bodies, where mRNA is degraded or stored ${ }^{35}$. Hypoxia caused a redistribution of both eIF4E and $4 E-T$ from predominantly cytoplasmic staining under aerobic conditions to substantial nuclear staining during hypoxia (Figure $5 \mathrm{~A}-\mathrm{C}$ ). This redistribution occurred progressively over time in hypoxic conditions, correlating with the gradual dephosphorylation of 4E-T (Figure 5D). In addition, hypoxic cells exhibit significant eIF4E and $4 \mathrm{E}-\mathrm{T}$ staining in the perinuclear area, which may be associated with the nuclear envelope or the ER. Interestingly, hypoxia also increased the number of $4 \mathrm{E}-\mathrm{T}$ speckles, which have been described as P-bodies ${ }^{36}$.

\section{Gene-specific regulation of translation}

As translation efficiency is highly gene specific, we anticipated that individual genes would show different patterns of translation efficiency during acute and prolonged hypoxia. To investigate this, we fractionated polysomal mRNA and subsequently measured the mRNA abundance of 
individual genes by quantitative RT-PCR (Figure 6A). We first confirmed that concomitant with an increase in polysome association, the non/ subpolysomal abundance decreased (Supplementary Figure S2).

A

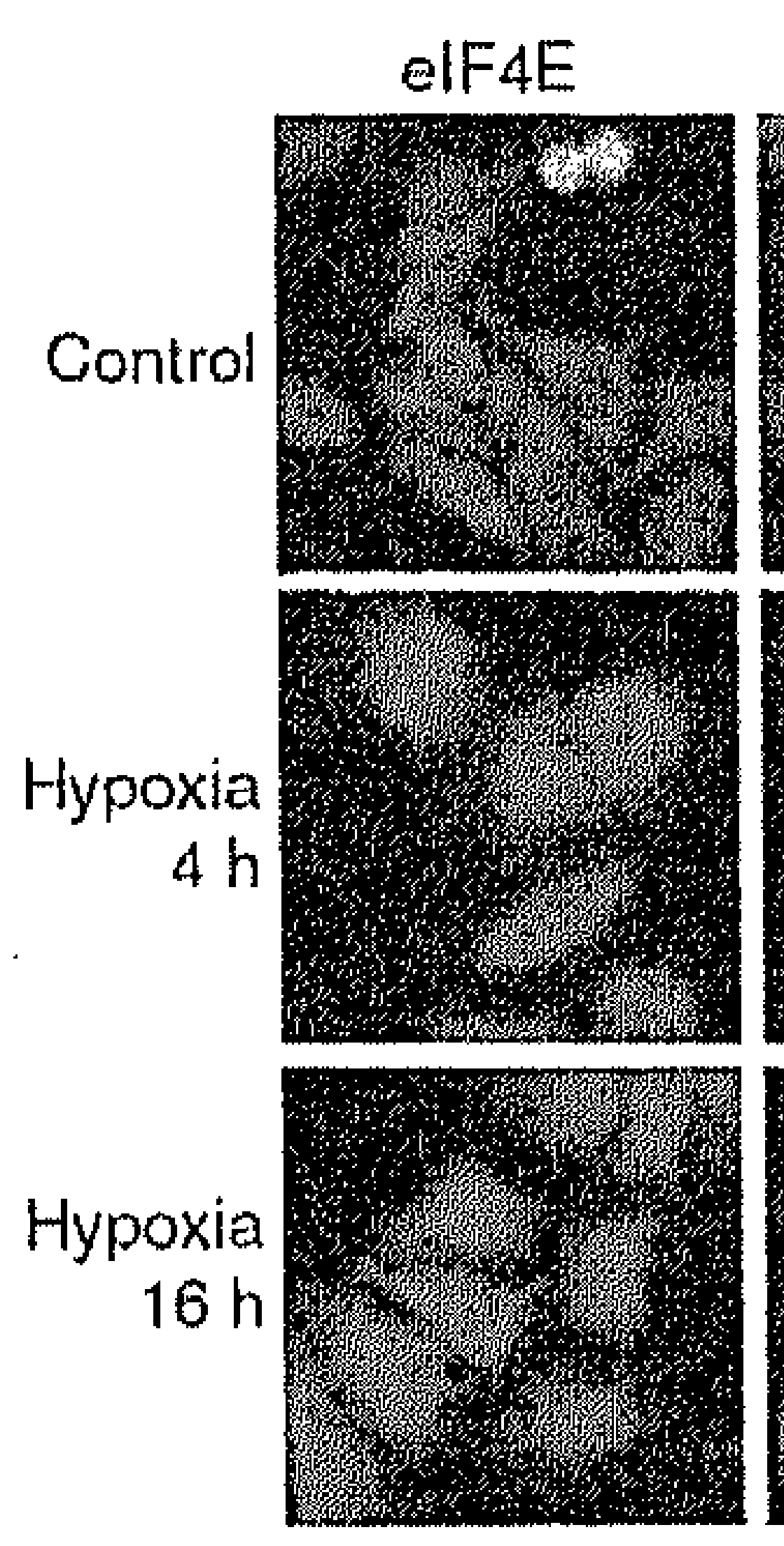

B
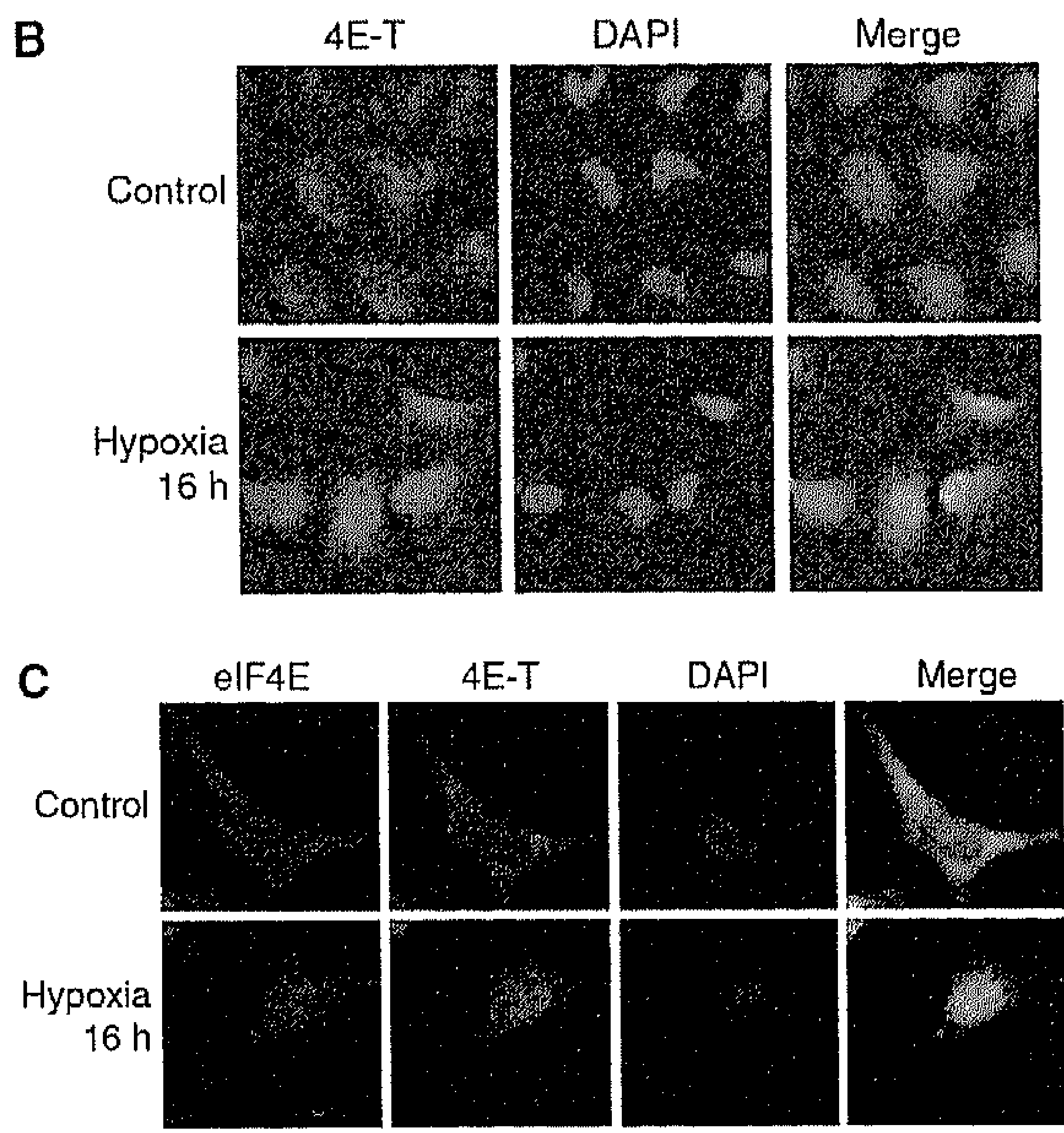

D

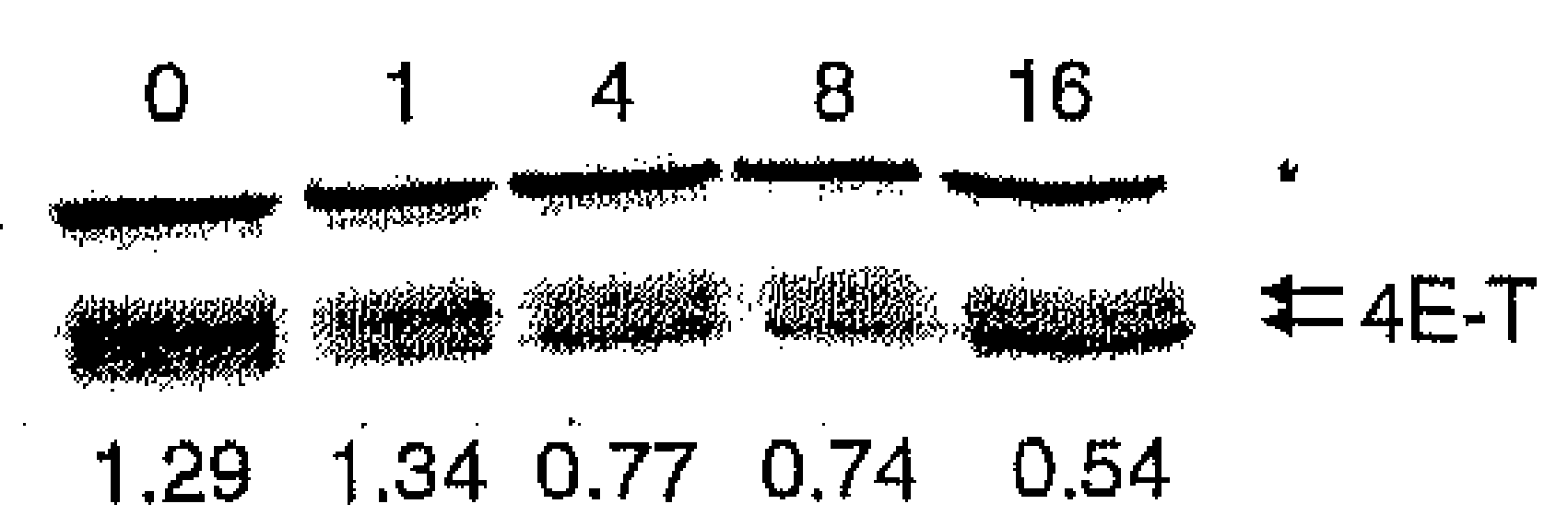

Figure 5. 4E-Tand eIF4E relocalize during hypoxia. HeLa cells were treated with $0.0 \%$ $\mathrm{O}_{2}$ for $0-16$ h. Cells were stained with DAPI and (A) a polyclonal antibody against eIF4E, (B) a polyclonal antibody against 4E-T or (C) a monoclonal antibody against eIF4E and a polyclonal antibody against 4E-T. Cells were visualized by confocal microscopy and individual pictures merged to determine colocalization. (D) Cell lysates were separated by SDS-PAGE and immunoblots performed using antibodies against $4 E-T$. The ratio of the individual bands was quantified with optical densitometry. A crossreacting band is indicated $(*)$. 
Subsequently, we quantified both the transcript recruitment and distribution within the polysomes (expressed as the relative fraction of translated transcripts and the average number of ribosomes per translated transcript, respectively).

We first measured the translational profile of the housekeeping gene $\beta$ actin (Figure 6B). In aerobic cells, it is efficiently translated with a majority of the mRNA in polysome fractions 5 and 6 . After $1 \mathrm{~h}$ of hypoxia, there is a marked reduction in translation, as evidenced by a shift toward the lower polysome fractions, which recovers considerably by $16 \mathrm{~h}$. The drop in translation efficiency at $1 \mathrm{~h}$ is due to reductions in the relative fraction of translated mRNA and in the average number of ribosomes per translated transcript (Figure 6B). At later time points, only the average number of ribosomes per transcript remained low. The kinetic changes in translation efficiency for $\beta$-actin are similar to those observed for overall translation efficiency.

Many proteins are induced at the transcriptional level by hypoxia and we suspected that these genes might be preferentially translated during hypoxia. We investigated the translation of the HIF-1 target gene carbonic anhydrase IX (CAIX), which is important for tumor cell growth and survival during hypoxia ${ }^{37}$. Figure $6 \mathrm{C}$ shows an $\sim 500$-fold transcriptional induction of CAIX during hypoxia. Polysome analysis indicates that, similar to $\beta$-actin, CAIX is initially efficiently translated but severely inhibited after $1 \mathrm{~h}$ of hypoxia. A significant restoration of the polysome distribution occurs after $16 \mathrm{~h}$ and thus ensures protein mRNA. As for $\beta$-actin, the initial inhibition of CAIX translation is due to a drop in the recruitment of the mRNA into polysomes and in the number synthesis at this time where there is also significantly more cellular of ribosomes per transcript. However, during prolonged hypoxia, the mRNA recruitment recovers and lower translation efficiency is attributed only to a small reduction in the average number of ribosomes per transcript. 
A

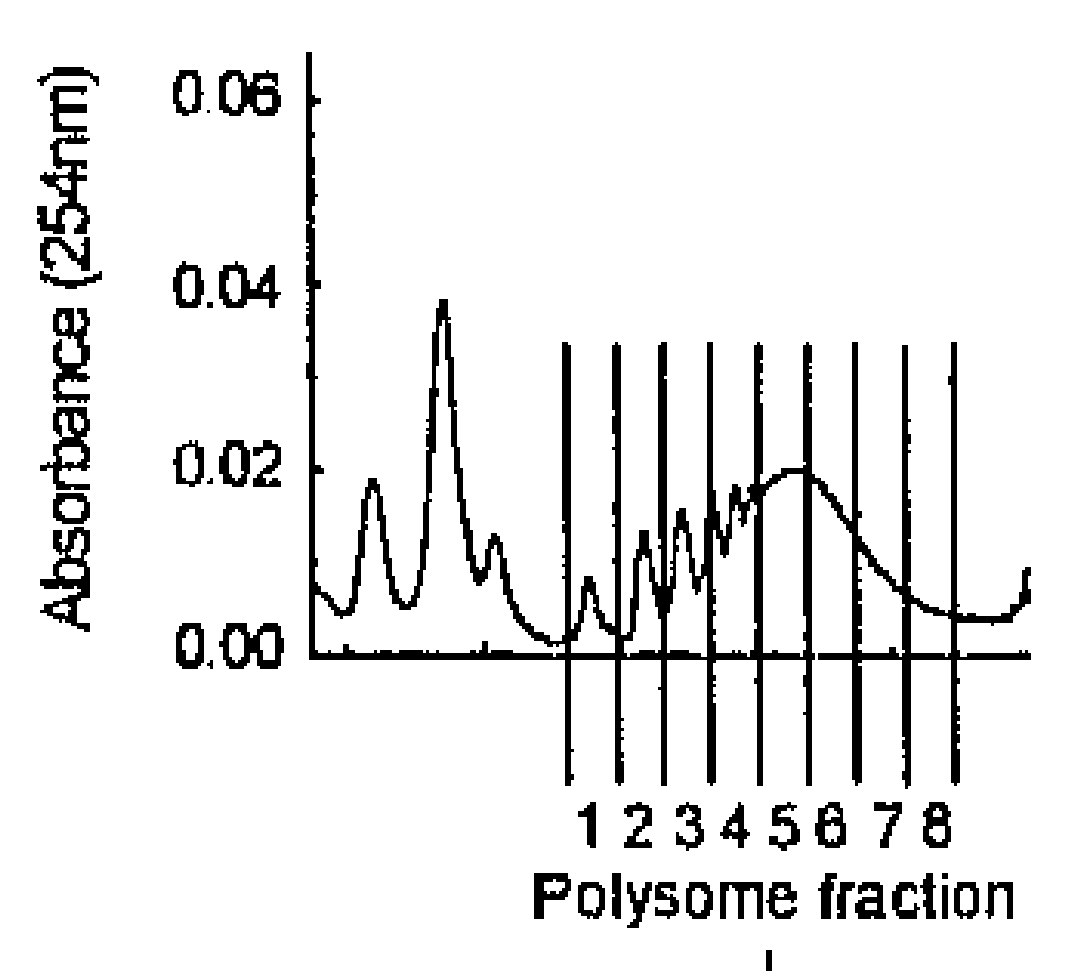

B

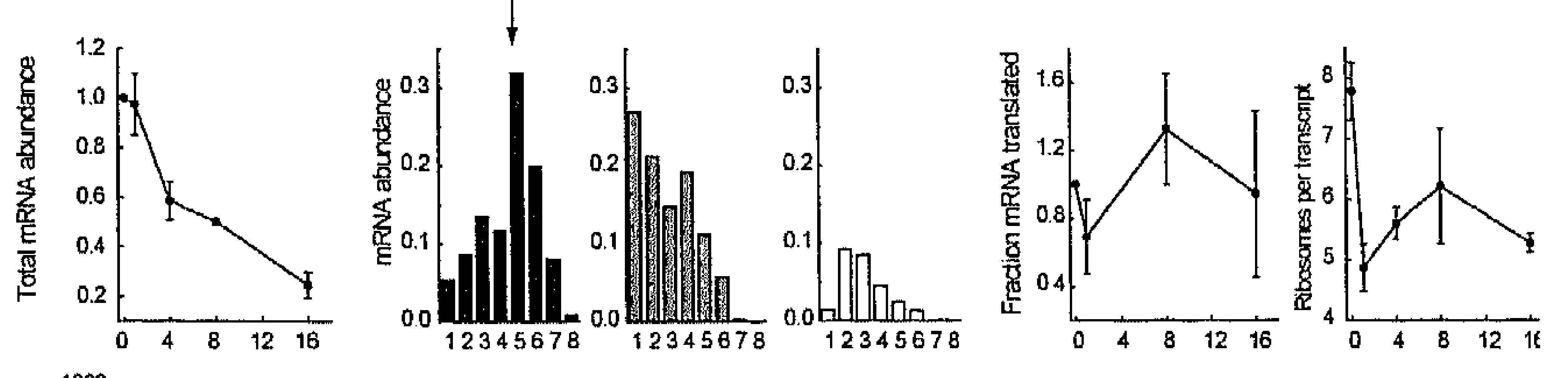

C
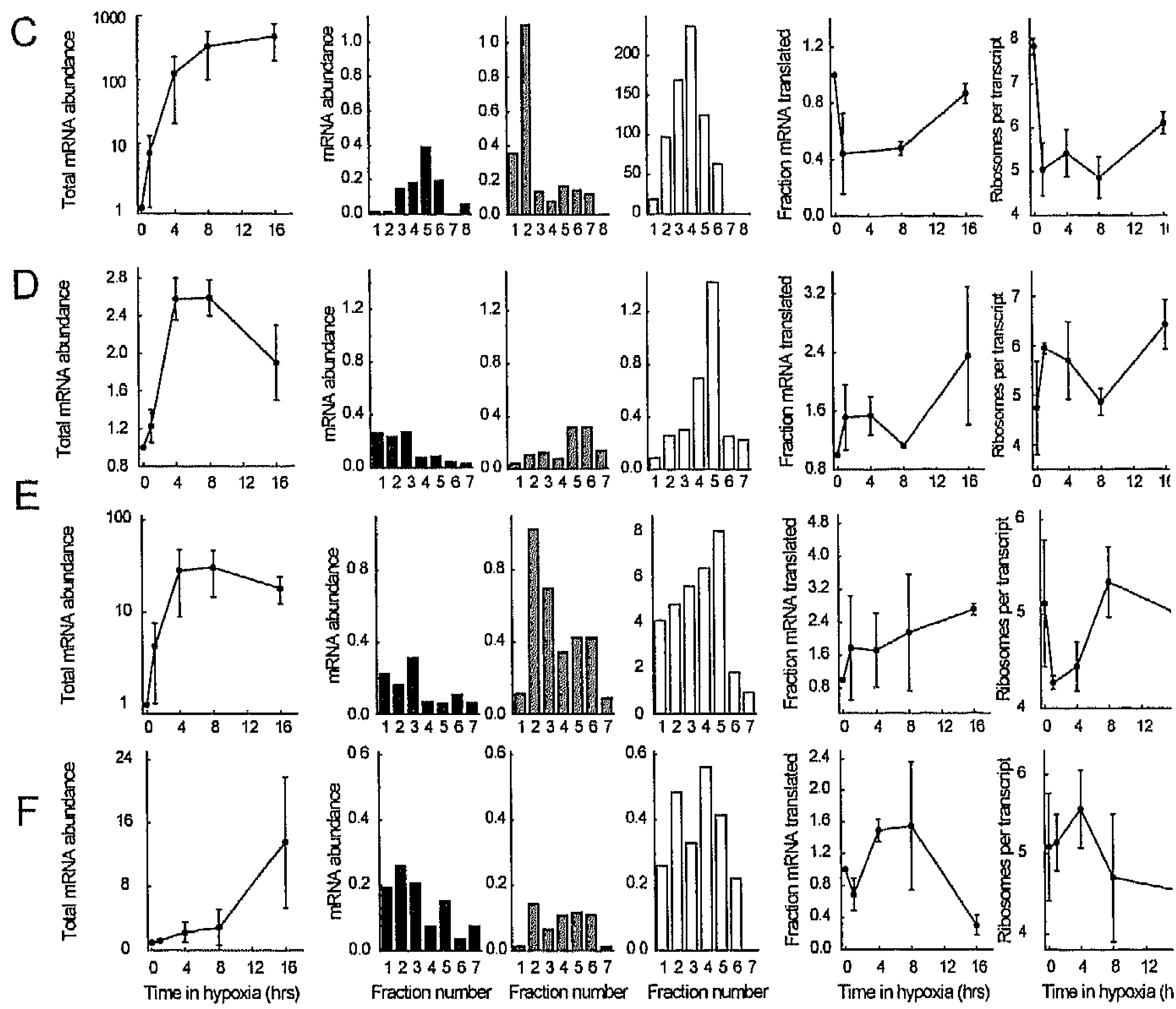
Figure 6. Gene-specific regulation of translation during hypoxia. HeLa cells were exposed to $0.0 \% \mathrm{O}_{2}$ for $0-16 \mathrm{~h}$ and cell lysates were separated on sucrose gradients. (A) Fractions were collected as indicated, RNA was isolated and reverse transcribed. Thereafter, the total mRNA abundance of (B) $\beta$-actin, (C) CAIX, (D) ATF4, (E) CHOP and (F) GADD34 wasdetermined using real-time quantitative PCR. The left panel shows total mRNA levels from unfractionated samples, normalized by $18 S$ rRNA signal. The following three panels use black, gray and white bars to represent the gene abundance in polysome fractions following 0,1 or $16 \mathrm{~h}$ hypoxia, respectively. The last two graphs show components of translation efficiency. This includes the relative fraction of transcripts in polysomes (i.e. corrected for total mRNA abundance) and the average number of ribosomes per mRNA. Graphs show the average from two independent experiments, and the histograms show the results from one representative experiment.

ATF4 is a central transcription factor mediating the UPR following ER stress. Both thapsigargin (which causes ER stress) and $16 \mathrm{~h}$ of hypoxia result in eIF2 $\alpha$ phosphorylation and translational induction of ATF4 in a PERK-dependent manner ${ }^{13}, 17,38$. Under normal conditions, the translation efficiency of this gene is low, with most of the mRNA found in fractions 13 (Figure 6D). In direct contrast to $\beta$-actin and CAIX, its translation is substantially increased during acute hypoxia, due to increased recruitment into the polysomes and an increase in the average number of ribosomes per transcript. In agreement with Blais et $\mathrm{a}^{38}$, we also observed a further increase in ATF4 translation efficiency during prolonged hypoxia.

An important transcriptional target of ATF4 is the C/EBP transcription factor $\mathrm{CHOP}^{39}$, which induces cell cycle arrest and apoptosis during $\mathrm{ER}$ stress. Figure $6 \mathrm{E}$ shows that $\mathrm{CHOP}$ is regulated both transcriptionally and translationally by hypoxia. Translation is only moderately inhibited during acute hypoxia, as shown by a drop in the average number of ribosomes per transcript. However, this reduction is much smaller than average overall reduction (Figure $1 \mathrm{C}$ ) and the reductions observed for both $\beta$-actin and CAIX. After $16 \mathrm{~h}$ of hypoxia, translation of CHOP is stimulated, as indicated by a recovery in the number of ribosomes per transcript and a marked increase in the fraction of translated mRNA. Recovery from ER stress requires the GADD34 gene, which is induced in a PERK-dependent ${ }^{40}$ 
and CHOP-dependent ${ }^{41}$ manner. GADD34 stimulates the activity of PP1C to dephosphorylate eIF 2 . We found that, like CHOP, GADD 34 is regulated both transcriptionally and translationally during hypoxia. Interestingly, its translation efficiency is highest after $4 \mathrm{~h}$ of hypoxia, which coincides with the start of recovery from eIF2" phosphorylation and overall translation inhibition (Figure 6F). In contrast, GADD34 mRNA is unable to completely bypass the translation inhibition after $16 \mathrm{~h}$.

\section{Gene-specific regulation of translation dependence upon eIF 2 "}

The gene-specific changes in translation noted above likely reflect the underlying eIF $2 \alpha$ and eIF4F-dependent mechanisms of translation control during hypoxia. We thus analyzed gene-specific translation in WT and S51A MEFs to establish the dependence of individual genes on eIF2 $\alpha$ regulation (Figure 7). In contrast to WT MEFs, S51A MEFs show no loss in translation efficiency of $\beta$-actin or CAIX during acute hypoxia (Figure 7A and $B$ ). The loss in translation efficiency for these genes in the WT cells is similar to that observed in HeLa cells and is due primarily to a reduction in the average number of ribosomes per transcript. For acute hypoxia, the translation efficiency during prolonged hypoxia is similar for these two genes in both cell lines. For ATF4 (Supplementary Figure S2), CHOP (data not shown) and GADD34 (Figure 7C), acute both these genes, S51A MEFs show virtually no reduction in this parameter during the acute phase of hypoxia. However, in contrast to hypoxia causes a stimulation of translation in WT MEFs that is similar to HeLa cells. However, the translational induction is entirely absent in S51A MEFs. The increase in translation efficiency for GADD34 in WT MEFs during acute hypoxia results mainly from an increase in the average number of ribosomes per transcript (Figure $7 C$ ). Cells that are defective in eIF $2 \alpha$ phosphorylation show impaired regulation of this parameter. Thus, for all genes examined, 
the observed changes in translation efficiency during acute hypoxia are dependent on eIF $2 \alpha$ phosphorylation.

\section{Discussion}

Rapid and persistent downregulation of protein synthesis is thought to be a means of energy preservation and to protect against the lethal effects of hypoxia ${ }^{10,42}$. Here we show that the inhibition of global mRNA translation during hypoxia exhibits a biphasic response (Figure 8 ). The initial rapid inhibition (i.e. 15 min-4 h) is primarily dependent on eIF2 $\alpha$ phosphorylation, whereas inhibition during prolonged hypoxia is independent of eIF $2 \alpha$. Phosphorylation of eIF $2 \alpha$ under conditions of anoxia is extremely rapid, occurring almost as quickly as we can establish hypoxia in our system (15-30 $\mathrm{min}$ ). We have previously shown that eIF2 $\alpha$ is also phosphorylated under more moderate hypoxic conditions, although to a smaller degree and after longer times ${ }^{10}$. We speculate that this rapid anoxic response may be especially important during the acute exposures to hypoxia/anoxia that frequently occur in tumors due to the transient opening and closing of blood vessels. This rapid response may explain the importance of eIF $2 \alpha$ and ATF 4 in the tolerance of cells to oxidative stress, which also occurs during hypoxia/ reoxygenation $\mathrm{cycles}^{20}$. This hypothesis is supported by a recent study by $\mathrm{Bi}$ et al, who showed that activation of the PERK-eIF2 $\alpha$ pathway during hypoxia contributes to overall tumor growth ${ }^{13}$. Human tumor cells expressing a dominant-negative PERK allele as well as MEFs lacking PERK or expressing the S51A eIF2 $\alpha$ produce smaller tumors with increased cell death in hypoxic areas than their WT counterparts ${ }^{13}$. Thus, although activation of eIF $2 \alpha$ phosphorylation in response to hypoxia is transient, this response appears critical for longterm cell survival within hypoxic regions of tumors. 


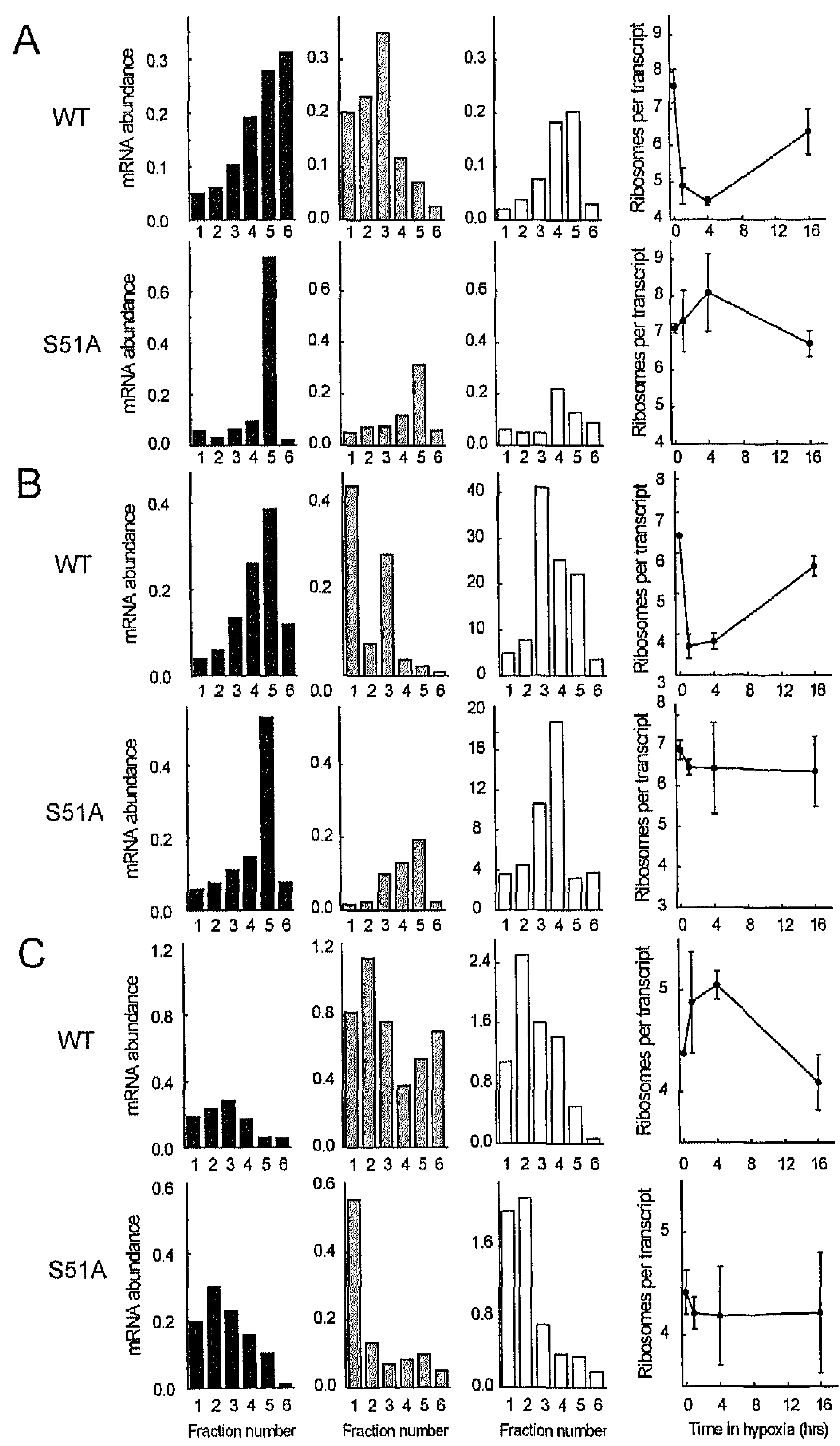


Figure 7. Dependence on eIF $2 \alpha-P$ for gene-specific translational regulation. WT and S51A MEFs were exposed to $0.0 \% \mathrm{O}_{2}$ for $0-16 \mathrm{~h}$ and cell lysates were separated on sucrose gradients. RNA was isolated from polysome fractions and reverse transcribed. Thereafter, the abundance of (A) $\beta$-actin, (B) CAIX and (C) GADD34 was determined using real-time quantitative PCR. The first three panels use black, solid gray, hatched grey and white bars to represent the gene abundance in polysome fractions following $0,1,4$ or $16 \mathrm{~h}$ hypoxia, respectively. The following graph depicts one component of translation efficiency, that is, the average number of ribosomes per mRNA.

Inhibition of translation during prolonged anoxia is associated with disruption of the mRNA cap-binding complex eIF4F and sequestration of eIF4E by both dephosphorylated 4E-BP1 and 4E-T. To our knowledge, this report is the first to show a physiological stress-induced regulation of the localization of eIF4E and its transporter $4 E-T$. Accumulation of eIF4E in the cell nucleus or P-bodies renders it unavailable for cytoplasmic translation. Relocalization of eIF4E may have additional roles apart from reducing capdependent protein translation, including effects on mRNA processing, transport and degradation. Moderate hypoxia ( $1 \%$ ) has also been shown to affect the eIF4F complex through inhibition of $\mathrm{mTOR}^{30}$. However, the kinetics and relative contribution of the eIF4F pathway on inhibition of global and gene-specific translation during more moderate hypoxia remain to be determined (see below).

Analysis of mRNA distribution within polysomes at different times also revealed interesting mechanistic differences during acute and prolonged hypoxia. Acute hypoxia caused a substantial drop in the average number of ribosomes per translated transcript. This presumably results from a reduction in the rate at which ribosomal subunits can be loaded onto mRNA, as each subunit requires a new nonphosphorylated eIF2 $\alpha$ molecule. Inhibition of translation during prolonged hypoxia via eIF4F did not show this effect. Instead, translation was suppressed mainly by reducing the fraction of mRNA found within the polysomes. These results are consistent with a model in which the eIF4F cap-binding complex remains bound to the mRNA allowing sequential rounds of initiation by 
available eIF $2 \alpha$ complexes. When translation is inhibited via eIF4F, many transcripts will lack this cap binding complex and hence will not be competent to initiate translation. However, those transcripts that do contain eIF4F will be translated normally (Figure 8). A similar phenomenon has been observed for a subpopulation of mRNAs that contains $5^{\prime}$ terminal oligopyrimidine tracts (5'TOPs), which alternate between translationally repressed and active states in response to various stimuli (reviewed $\mathrm{in}^{43}$ ). It is thus conceivable that the overall reduction in mRNA translation observed during prolonged hypoxia affects a subset of genes, such as those with 5'TOPs, to a greater degree than others.

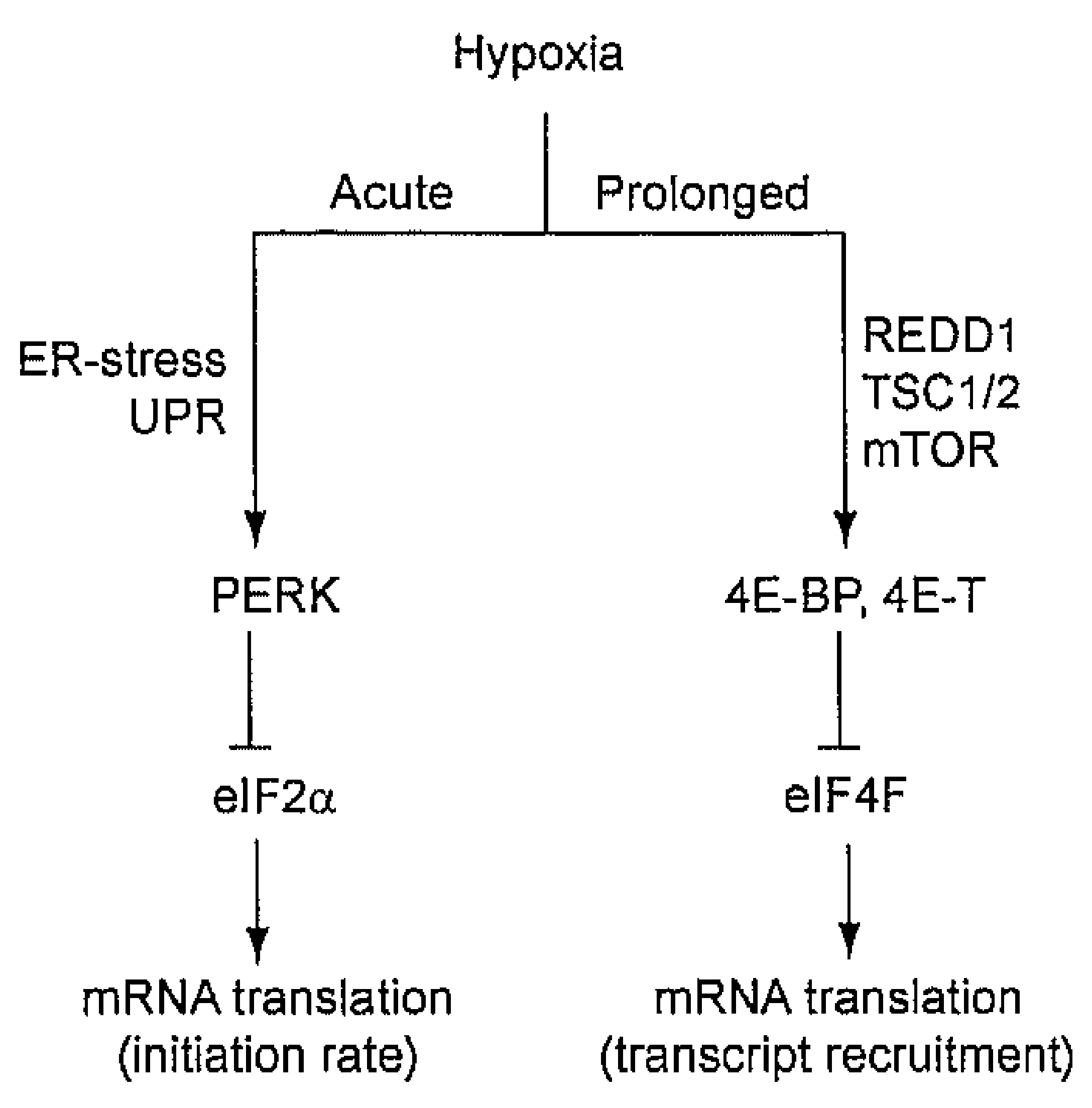

Figure 8 Model of the effects of hypoxia on overall mRNA translation. Acute hypoxia causes transient eIF2a phosphorylation due to PERK activation as a part of the UPR. This results in inhibition of the rate of translation initiation. Following prolonged hypoxic conditions, activation of $4 \mathrm{E}-\mathrm{BP}$ and $4 \mathrm{E}-\mathrm{T}$ causes disruption of eIF4F, which inhibits the recruitment of mRNA to polysomes. Both molecular mechanisms affect specific mRNAs to varying degrees, resulting in differential gene expression.

An important issue that arises from our study is the nature of the oxygensensing pathways upstream of eIF $2 \alpha$ and eIF4F. Substantial evidence suggests that the oxygen sensors are largely independent of the HIF oxygen-sensing pathway ${ }^{10,44}$. In the case of eIF $2 \alpha$, its phosphorylation occurs in an HIF-independent manner. Instead, it requires PERK activation ${ }^{10}$ and is associated with activation of the UPR in response to ER stress $^{13,45}$. The upstream signaling that leads to eIF4F disruption is less clear, with perhaps both HIF-dependent and independent components. Hypoxia has been shown to prevent insulin stimulation of mTOR and 
phosphorylation of its substrate 4E-BP1 during conditions of moderate hypoxia and serum starvation ${ }^{30}$. Similarly, Brugarolas et al showed that induction of REDD1 during hypoxia resulted in activation of the mTOR inhibitory complex TSC1/TSC2 ${ }^{46}$. As we also observe a decrease in the phosphorylation of 4E-BP1 after prolonged hypoxia, the eIF4F-dependent changes in translation reported here may also be due in part to inhibition of mTOR via REDD1 and TSC1/2. However, it is unlikely that this accounts entirely for eIF4F disruption and translation inhibition during hypoxia. REDD1 is an HIF-dependent gene and both mTOR inhibition and translation inhibition during hypoxia occur in HIFla-knockout cells ${ }^{10,} 30$. Furthermore, our data indicate that eIF4F disruption occurs before substantial binding of eIF4E to 4E-BP1. Here we have identified redistribution of eIF4E into the cell nucleus via $4 \mathrm{E}-\mathrm{T}$ as an additional mechanism for eIF4F disruption during hypoxia. Further work will be needed to establish to what degree inhibition of translation is due to suppression of mTOR/4E-BP1 phosphorylation and 4E-T activation, as well as to the requirements of HIF in both of these pathways.

The fact that both eIF $2 \alpha$ and eIF4F independently affect translation during hypoxia has important implications for the regulation of gene expression. mRNAs preferentially translated during acute hypoxia must be less dependent on eIF $2 \alpha$ availability, whereas mRNAs that are actively translated during prolonged hypoxia must be less dependent on eIF4F. The translation of ATF4, which contains two upstream open reading frames (UORFs) in its $5^{\prime} U T R$, is perhaps the best example of a mammalian gene that displays this type of preferential translation ${ }^{17}$. When eIF2 $\alpha$ availability is high, translation begins at the $5^{\prime}$ most UORF and re-initiation occurs efficiently at the subsequent UORF, preventing translation from the correct start codon of ATF4. When eIF2 $\alpha$ is phosphorylated, there is a higher probability of skipping the second UORF and re-initiating at the bona fide start codon ${ }^{47}$. Here, we found that in addition to ATF4, the downstream genes CHOP and GADD34 are also translationally induced during acute hypoxia. The S51A MEFs, which are unable to phosphorylate 
eIF $2 \alpha$, are defective in this translational regulation. This result is consistent with a report showing that ER stress-induced expression of GADD34 protein can be prevented by keeping eIF2 $\alpha$ dephosphorylated ${ }^{40}$. The bypass of translation inhibition may thus facilitate the ability of GADD34 to dephosphorylate eIF2 $\alpha$ and promote recovery from ER stress.

Our results predict that during prolonged hypoxia, gene transcripts with lower dependency on eIf4F should be preferentially translated. The translation of ATF4 and CHOP was in fact stimulated in HeLa cells after 16 $h$ of hypoxia when the eIF4F complex was disrupted. Preferential translation under conditions of limiting cap-binding complex activity can occur through a higher than average affinity for eIF $4 \mathrm{~F}^{48}$. Another group of mRNAs that can be translated independently of the eIF4F complex are those that contain an internal ribosomal entry site (IRES) in their $5^{\prime} U_{T R}{ }^{22,}$ 49. The presence of an IRES enables translation initiation under conditions where eIF4F-dependent translation is inhibited. Importantly, both mouse HIF- $1 \alpha$ and VEGF have been shown to contain functional IRESs within their $5^{\prime}$ UTR, although their biological importance is not yet firmly established ${ }^{50,51}$. This provides a mechanism to ensure their translation during prolonged hypoxia where eIF4F is disrupted. The mechanism responsible for the selective translation of ATF4 and CHOP during prolonged hypoxia remains to be identified.

In conclusion, we have shown that mRNA translation is inhibited through multiple independent pathways with differing activation kinetics during hypoxia. These distinct modes of translational control influence the translation of individual genes to varying degrees and consequently can influence hypoxia-regulated protein expression in complex ways. An important finding is that inhibition of translation via eIF2 $\alpha$ is transient, leading to dynamic changes in the translation efficiency of genes over the first $8 \mathrm{~h}$ of hypoxia. Our selected analysis of gene translation during hypoxia suggests that many genes may be differentially regulated by hypoxia. A complete survey of the genome for differentially translated genes during various exposures to hypoxia and their dependence of eIF2 $\alpha$ 
is possible and will undoubtedly identify novel and important hypoxiaregulated proteins ${ }^{11}$.

\section{Acknowledgements}

This work was financially supported by Netherlands Organization for Scientific Research (NWO), the Dutch Cancer Society (KWF Kankerbestrijding) and the Euroxy grant from the 6th framework of the EU to BGW, from the Norwegian Research Council to MK and from grant CA94214 from the National Institutes of Health (NIH) to CK.

\section{References}

1. Harris AL: Hypoxia--a key regulatory factor in tumour growth, Nat Rev Cancer 2002, 2:38-47

2. Wouters BG, Weppler SA, Koritzinsky M, Landuyt W, Nuyts S, Theys J, Chiu RK, Lambin P: Hypoxia as a target for combined modality treatments, Eur J Cancer 2002, 38:240-257

3. Graeber TG, Osmanian C, Jacks T, Housman DE, Koch CJ, Lowe $S W$, Giaccia AJ: Hypoxia-mediated selection of cells with diminished apoptotic potential in solid tumours, Nature 1996, 379:88-91

4. Semenza GL: Targeting HIF-1 for cancer therapy, Nat Rev Cancer 2003, 3:721-732

5. Ryan HE, Lo J, Johnson RS: HIF-1 alpha is required for solid tumor formation and embryonic vascularization, Embo J 1998, 17:3005-3015

6. Tang N, Wang L, Esko J, Giordano FJ, Huang Y, Gerber HP, Ferrara N, Johnson RS: LosS of HIF-1alpha in endothelial cells disrupts a hypoxia-driven VEGF autocrine loop necessary for tumorigenesis, Cancer Cell 2004, 6:485-495

7. Leek RD, Stratford I, Harris AL: The role of hypoxia-inducible factor-1 in three-dimensional tumor growth, apoptosis, and regulation by the insulinsignaling pathway, Cancer Res 2005, 65:4147-4152

8. Bennewith KL, Durand RE: Quantifying transient hypoxia in human tumor xenografts by flow cytometry, Cancer Res 2004, 64:6183-6189

9. Cardenas-Navia LI, Yu D, Braun RD, Brizel DM, Secomb TW, Dewhirst MW: Tumor-dependent Kinetics of Partial Pressure of Oxygen Fluctuations during Air and Oxygen Breathing, Cancer Res 2004, 64:6010-6017

10. Koumenis C, Naczki C, Koritzinsky M, Rastani S, Diehl A, Sonenberg N, Koromilas A, Wouters BG: Regulation of protein synthesis by hypoxia via activation of the endoplasmic reticulum kinase PERK and phosphorylation of the translation initiation factor eIF2alpha, Mol Cell Biol 2002, 22:7405-7416

11. Koritzinsky $M$, Seigneuric $R$, Magagnin $M G$, van den Beucken $T$, Lambin $P$, Wouters BG: The hypoxic proteome is influenced by gene-specific changes in mRNA translation, Radiother Oncol 2005, 76:177-186

12. Wouters BG, Koritzinsky M, Chiu RK, Theys J, Buijsen J, Lambin P: Modulation of cell death in the tumor microenvironment, Semin Radiat Oncol 2003, $13: 31-41$

13. Bi M, Naczki C, Koritzinsky M, Fels D, Blais J, Hu N, Harding H, Novoa I, Varia M, Raleigh J, Scheuner D, Kaufman RJ, Bell J, Ron D, Wouters BG, Koumenis 
C: ER stress-regulated translation increases tolerance to extreme hypoxia and promotes tumor growth, Embo J 2005, 24:3470-3481

14. Erler JT, Cawthorne $\mathrm{CJ}$, Williams KJ, Koritzinsky M, Wouters BG, Wilson C, Miller C, Demonacos C, Stratford IJ, Dive C: Hypoxia-mediated downregulation of Bid and Bax in tumors occurs via hypoxia-inducible factor 1dependent and -independent mechanisms and contributes to drug resistance, Mol Cell Biol 2004, 24:2875-2889

15. Lefebvre $V H$, Van Steenbrugge $M$, Beckers $V$, Roberfroid $M$, Buc-Calderon $P$ : Adenine nucleotides and inhibition of protein synthesis in isolated hepatocytes incubated under different pO2 levels, Arch Biochem Biophys 1993, 304:322331

16. Johannes G, Carter MS, Eisen MB, Brown PO, Sarnow P: Identification of eukaryotic mRNAs that are translated at reduced cap binding complex eIF4F concentrations using a cDNA microarray, Proc Natl Acad Sci U S A 1999, 96:13118-13123

17. Harding HP, Novoa I, Zhang $Y$, Zeng $H$, Wek R, Schapira M, Ron' D: Regulated translation initiation controls stress-induced gene expression in mammalian cells, Mol Cell 2000, 6:1099-1108

18. Holland EC, Sonenberg N, Pandolfi PP, Thomas G: Signaling control of mRNA translation in cancer pathogenesis, Oncogene 2004, 23:3138-3144

19. Schroder M, Kaufman RJ: The mammalian unfolded protein response, Annu Rev Biochem 2005, 74:739-789

20. Harding HP, Zhang $Y$, Zeng $H$, Novoa I, Lu PD, Calfon M, Sadri N, Yun C, Popko B, Paules R, Stojdl DF, Bell JC, Hettmann T, Leiden JM, Ron D: An integrated stress response regulates amino acid metabolism and resistance to oxidative stress, Mol Cell 2003, 11:619-633

21. Gebauer F, Hentze MW: Molecular mechanisms of translational control, Nat Rev Mol Cell Biol 2004, 5:827-835

22. Holcik M, Sonenberg N: Translational control in stress and apoptosis, Nat Rev Mol Cell Biol 2005, 6:318-327

23. Hay N, Sonenberg N: Upstream and downstream of mTOR, Genes Dev. 2004, 18:1926-1945

24. DeGracia DJ, Kumar R, Owen CR, Krause GS, White BC: Molecular pathways of protein synthesis inhibition during brain reperfusion: implications for neuronal survival or death, J Cereb Blood Flow Metab 2002, 22:127-141

25. Kumar R, Azam S, Sullivan JM, Owen $C$, Cavener DR, Zhang $P$, Ron D, Harding HP, Chen JJ, Han A, White BC, Krause GS, DeGracia DJ: Brain ischemia and reperfusion activates the eukaryotic initiation factor 2alpha kinase, PERK, J Neurochem 2001, 77:1418-1421

26. Neumar RW, DeGracia DJ, Konkoly LL, Khoury JI, White BC, Krause GS: Calpain mediates eukaryotic initiation factor $4 \mathrm{G}$ degradation during global brain ischemia, J Cereb Blood Flow Metab 1998, 18:876-881

27. Martin de la Vega C, Burda J, Nemethova M, Quevedo C, Alcazar A, Martin ME, Danielisova $V$, Fando JL, Salinas $M$ : Possible mechanisms involved in the down-regulation of translation during transient global ischaemia in the rat brain, Biochem J 2001, 357:819-826

28. Martin ME, Munoz FM, Salinas M, Fando JL: Ischaemia induces changes in the association of the binding protein 4E-BP1 and eukaryotic initiation factor (eIF) $4 G$ to eIF4E in differentiated PC12 cells, Biochem J 2000, 351 Pt 2:327-334

29. Tinton SA, Buc-Calderon PM: Hypoxia increases the association of 4E-binding protein 1 with the initiation factor $4 \mathrm{E}$ in isolated rat hepatocytes, FEBS Lett $1999,446: 55-59$ 
30. Arsham AM, Howell 3], Simon MC: A Novel Hypoxia-inducible Factorindependent Hypoxic Response Regulating Mammalian Target of Rapamycin and Its Targets, J. Biol. Chem. 2003, 278:29655-29660

31. Kimball SR JL: Regulation of translation initiation in mammalian cells by amino acids. Edited by Sonenberg N HJ, Mathews MB. Cold Spring Harbor, NY: Cold Spring Harbor Laboratory Press, 2000, p

32. Scheuner D, Song B, McEwen E, Liu C, Laybutt R, Gillespie P, Saunders $T$, Bonner-Weir S, Kaufman RJ: Translational control is required for the unfolded protein response and in vivo glucose homeostasis, Mol Cell 2001, 7:11651176.

33. Lejbkowicz F, Goyer C, Darveau A, Neron S, Lemieux R, Sonenberg N: A fraction of the mRNA $5^{\prime}$ cap-binding protein, eukaryotic initiation factor $4 \mathrm{E}_{\text {, }}$ localizes to the nucleus, Proc Natl Acad Sci U S A 1992, 89:9612-9616

34. Dostie 3, Ferraiuolo $M$, Pause A, Adam SA, Sonenberg $N$ : A novel shuttling protein, 4E-T, mediates the nuclear import of the mRNA $5^{\prime}$ cap-binding protein, eIF4E, Embo J 2000, 19:3142-3156

35. Andrei $M A$, Ingelfinger $D$, Heintzmann $R$, Achsel $T$, Rivera-Pomar $R$, Luhrmann $\mathrm{R}$ : A role for eIF4E and eIF4E-transporter in targeting mRNPs to mammalian processing bodies, Rna 2005, 11:717-727

36. Ferraiuolo MA, Basak S, Dostie J, Murray EL, Schoenberg DR, Sonenberg N: A role for the eIF4E-binding protein 4E-T in P-body formation and mRNA decay, J Cell Biol 2005, 170:913-924

37. Robertson N, Potter C, Harris AL: Role of carbonic anhydrase IX in human tumor cell growth, survival, and invasion, Cancer Res 2004, 64:6160-6165

38. Blais JD, Filipenko $V, B i$, Harding $H P$, Ron D, Koumenis $C$, Wouters $B G$, Bell JC: Activating transcription factor 4 is translationally regulated by hypoxic stress, Mol Cell Biol 2004, 24:7469-7482

39. Fawcett TW, Martindale JL, Guyton KZ, Hai T, Holbrook NJ: Complexes containing activating transcription factor (ATF)/CAMP-responsive-elementbinding protein (CREB) interact with the CCAAT/enhancer-binding protein (C/EBP)-ATF composite site to regulate Gadd1.53 expression during the stress response, Biochem J 1999, 339 ( Pt 1):135-141

40. Novoa $I$, Zhang $Y$, Zeng $H$, Jungreis $R$, Harding HP, Ron D: Stress-induced gene expression requires programmed recovery from translational repression, Embo J 2003, 22:1180-1187

41. Marciniak SJ, Yun CY, Oyadomari S, Novoa I, Zhang $Y$, Jungreis R, Nagata $K$, Harding HP, Ron D: CHOP induces death by promoting protein synthesis and oxidation in the stressed endoplasmic reticulum, Genes Dev 2004, 18:30663077

42. Wouters $B G$, van den Beucken $T$, Magagnin $M G$, Koritzinsky $M$, Fels $D$, Koumenis $C$ : Control of the hypoxic response through regulation of $m R N A$ translation, Semin Cell Dev Biol 2005, 16:487-501

43. Meyuhas O HE: Translational control of TOP mRNAs. Edited by Sonenberg, N HJ, Mathews MB. 2000, 671-694 p

44. Jaakkola P, Mole DR, Tian YM, Wilson MI, Gielbert J, Gaskell SJ, Kriegsheim A, Hebestreit HF, Mukherji M, Schofield CJ, Maxwell PH, Pugh CW, Ratcliffe PJ: Targeting of HIF-alpha to the von Hippel-Lindau ubiquitylation complex by O2regulated prolyl hydroxylation, Science 2001, 292:468-472

45. Romero-Ramirez $L$, Cao $H$, Nelson D, Hammond $E$, Lee A-H, Yoshida $H$, Mori K, Glimcher LH, Denko NC, Giaccia AJ, Le Q-T, Koong AC: XBP1 Is Essential for Survival under Hypoxic Conditions and Is Required for Tumor Growth, Cancer Res 2004, 64:5943-5947 
46. Brugarolas 3, Let K, Hurley RL, Manning BD, Rening IH, Hafen E, Witters LA, Ellisen LW, Kaelin WG, Ir, Regulation of mTOR function in response to hypoxia by REDD1 and the TSC1/TSC2 tumor suppressor Complex, Genes Dev 2004, 18:2893-2904

47. Lu PD, Harding HP, Ron D: Translation reinitiation at alternative open reading frames regulates gene expression in an integrated stress response, 3 Cell Bol $2004,167: 27 \cdot 33$

48. Lawson TG, Cladaras MH, Ray BK, Lee KA, Abramson RD, Merrick WC, Thach RE: Discriminatory interaction of purified eukaryotic initiation factors AF plus $4 \mathrm{~A}$ with the $5^{\prime}$ ends of reovirus messenger RNAs, J Blol Chem 1988, 263:7266-7276

49. Carter MS KK, Sarnow P Cellular internal ribosome entry site elements and the use of CDNA microarrays in their investigation. Edited by Sonenberg $\mathrm{N} \mathrm{HJ}$, Mathews MB. Cold Spring Harbor, NY: Cold Spring Haror Laboratory Press, $2000,615-636 p$

50. Stein I, Itin A, Einat P, Skaliter R, Grossman Z, Keshet E: Translation of vascular endothelial growth factor MRNA by internal ribosome entry: implications for translation under hypoxa, Mol Cell Biol 1998, 18:3112-3119

51. Lang KJ, Kappel A, Goodall G1: Hypoxia-inducible factor-1alpha MRNA contains an internal ribosome entry site that allows efficient translation during normoxia and hypoxia, Mol Biol Cell 2002, 13:1792-1801 


\section{CHAPTER 3}

\section{Translational control of gene expression during hypoxia}

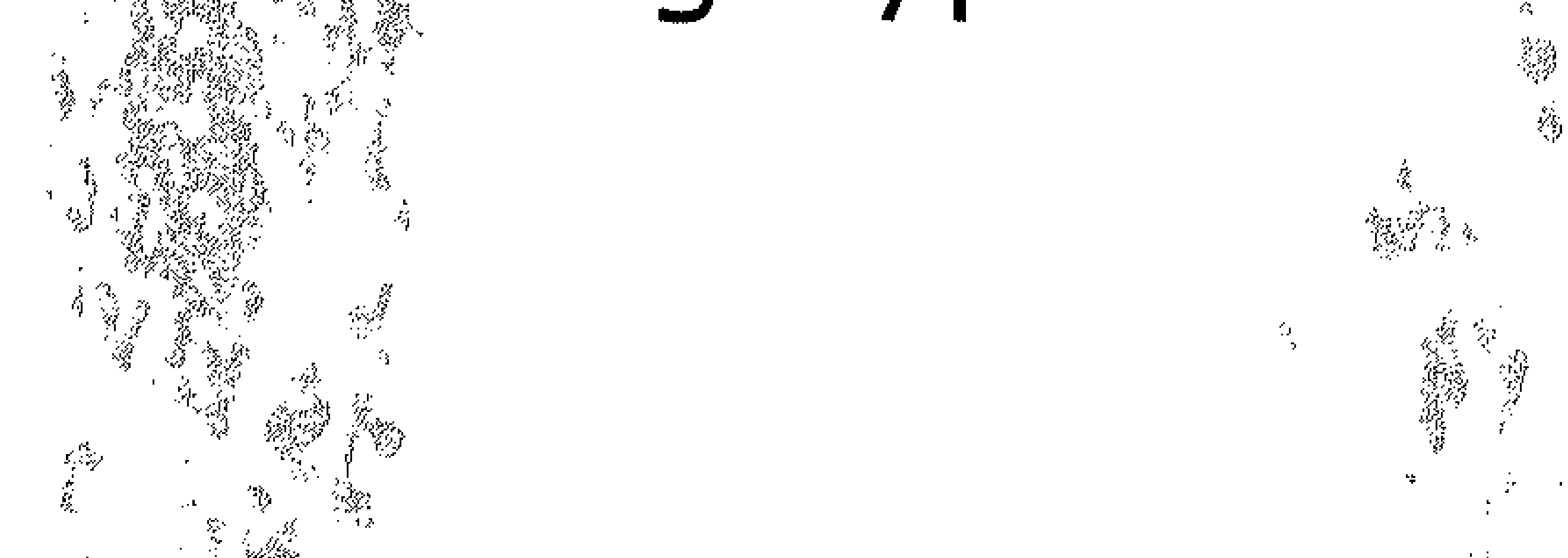

Cancer Biology \& Wherapy 2006 Jul; 5 (7):749-755.

Twan van den Beucken, Marianne Koritzinsky and Bradly G. Wouters. 


\section{Abstract}

Poor oxygenation is a unique and prevalent feature of solid tumors associated with poor patient prognosis. In part, this is caused by a series of adaptive cellular responses that together have a large impact on gene expression and cell phenotype. HIF plays a key role in this response by activating a transcriptional program that stimulates genes involved in angiogenesis, cell metabolism, cell survival and cell invasion. Recently, hypoxia has also been shown to suppress protein synthesis through the regulation of the initiation step of mRNA translation. This appears to be a common feature of the cell in response to hypoxia and is mediated by two distinct pathways. The first occurs rapidly, is transient, and is associated with activation of the unfolded protein response (UPR) that occurs in response to endoplasmic reticulum (ER) stress. Translation inhibition during this initial phase is due to phosphorylation of eukaryotic initiation factor $2 \alpha$ (eIF2 $\alpha$ ) in a PERK dependent manner. Although this effect is transient, overall levels of translation remain low during hypoxia due to inhibition of a second eukaryotic initiation complex, eIF4F. This second mechanism is multi-factorial, but due at least in part to inhibition of the mTOR kinase. Although each of these pathways leads to a general inhibition in translation, the consequence at the individual gene level is highly variable. This is due to sequences in the $5^{\prime}$ and $3^{\prime}$ untranslated regions (UTRs) of mRNA that confer their ability to maintain, or even increase, translation efficiency in spite of the overall inhibition. Consequently, regulation of mRNA translation appears to be an important mediator of gene expression during hypoxia. 


\section{Introduction}

It is well documented that the majority of solid tumors contain regions of poor oxygenation ${ }^{1}$. The oxygen concentration in these areas varies from complete anoxia to $\sim 4 \% \mathrm{O}_{2}$, while the range in normal tissues is $\sim 3-9 \%$ $\mathrm{O}_{2}$. There are several mechanisms that contribute to the formation of tumor hypoxia. As tumor cells proliferate, the oxygen demand on individual blood vessels increases until a point where the oxygen diffusion from the vessel becomes a limiting factor. This is referred to as 'chronic' hypoxia and can result in complete anoxia at distances of $\sim 150-200 \mu \mathrm{m}$ from the vessel wall. In addition, tumor cells also experience transient periods of low oxygen, often called 'acute' hypoxia due to transient changes in blood flow. This leads to areas of cycling oxygenation characterized by moderate to severe hypoxia dependent on the relative reduction in blood flow ${ }^{2}$. Therefore, large proportions of tumor cells are subject to hypoxic exposure and must be capable of adapting to this stress.

Clinical studies with oxygen electrodes and hypoxia markers have demonstrated not only that tumors are poorly oxygenated, but also that the level of hypoxia correlates with poor prognosis. This is true for patients treated by radiotherapy and chemotherapy, as might be expected due to the protection hypoxia offers against these treatments, and importantly also for patients treated by surgery alone $e^{3-5}$. High levels of hypoxia in primary tumors is also associated with an increased prevalence of metastasis ${ }^{5}, 6$. Together these data support the idea that hypoxia contributes to a more malignant tumor phenotype ${ }^{7}$.

Understanding the various cellular responses to hypoxia that are responsible for its negative impact on tumor biology is important in order to improve and/or develop effective anticancer therapies. Cellular responses to hypoxia can affect overall tumor growth and behavior in two distinct ways. First, they can contribute to 'hypoxic tolerance', the survival of the cell itself to the hypoxic stress. In minimally transformed cells, hypoxia leads to rapid cell death through the induction of p53 and 
apoptosis. Consequently, hypoxia provides an environment for the selective outgrowth of clones with reduced apoptotic potential. ${ }^{8}$ Second, assuming that the cells can survive, various hypoxia induced cellular responses alter gene expression and thus change the behavior of the cell.

The best described response to hypoxia is mediated by the hypoxia inducible factor (HIF) family of transcription factors (reviewed in ref. ${ }^{9}$ ). These transcription factors are activated primarily via posttranscriptional mechanisms during hypoxia and drive the expression of a large number of genes whose products are involved in angiogenesis, erythropoiesis, metabolic transition to anaerobic glycolysis, and cell motility and invasion. Consequently, HIF both promotes hypoxic cell tolerance through its ability to stimulate glycolysis and maintain ATP levels under conditions where oxidative phosphorylation is inhibited, and alters cell function in ways that can promote malignancy (e.g. stimulation of metastasis and angiogenesis).

\section{Regulation of mRNA translation}

In recent years, the regulation of mRNA translation has emerged as a second important mediator of the cellular response to hypoxia. Hypoxia causes a rapid and sustained inhibition in protein synthesis ${ }^{10}{ }^{11}$. This is primarily due to a HIF-independent oxygen sensing pathway that directly inhibits the initiation step of mRNA translation ${ }^{12,}{ }^{13}$. In a manner analogous to HIF, the inhibition of translation during hypoxia is hypothesized to be important through its ability to both promote cell survival and to alter gene expression and cell phenotype. Protein synthesis is a highly energy demanding process, and thus its inhibition during hypoxia promotes energy homeostasis and may sustain survival during this period of inefficient ATP production. Indeed, cells that are compromised in their ability to inhibit translation during hypoxia show increased apoptosis and reduced clonogenic survival to this stress ${ }^{14}$. However, suppression of mRNA translation can also induce important changes in protein expression. A general inhibition in protein synthesis 
may alter the relative levels of expressed proteins (proteome) due to differences in protein half-life. Furthermore, proteome changes are expected due to the fact that not all mRNA transcripts are affected to the same degree by the pathways that regulate translation during hypoxia. In some cases, the inhibition of overall mRNA translation can paradoxically induce the expression of certain proteins that are normally translationally repressed. Finally, because translation efficiency can be rapidly modulated, this mechanism allows for much faster protein changes than are possible through changes in transcription. In this review we focus our discussion on recent data describing both the mechanisms that control mRNA translation during hypoxia, and how they impact both overall and specific gene expression.

\section{Translation initiation}

Translation of eukaryotic mRNA transcripts generally begins with the assembly of the eukaryotic initiation factor $4 \mathrm{~F}$ (eIF4F) at the m7GpppN cap structure of the mRNA ${ }^{15}$. eIF4F consists of three subunits; the cap binding protein eIF4E, a scaffolding protein eIF4G and an ATP-dependent helicase eIF4A. Binding of eIF4F to a transcript facilitates the recruitment of the 435 pre-initiation complex, which is necessary to initiate translation. This complex is formed by the small $40 \mathrm{~S}$ ribosomal subunit, the initiation factor eIF3 and the ternary complex which consists of eIF2GTP and the methionine-loaded initiator tRNA (Met-tRNA). The 435 preinitiation complex scans through the $5^{\prime} U T R$ of the mRNA until it recognizes the AUG initiation codon. There it recruits the 605 ribosomal subunit to assemble the complete $80 \mathrm{~S}$ ribosome and starts the elongation phase of translation. Both the binding of the eIF4F complex to the mRNA as well as the formation and recruitment of the ternary complex form important regulatory control points during the translation initiation process in response to many forms of cells stress ${ }^{16}$. Furthermore, both of these control points are affected during hypoxic exposure. 


\section{Translational control during hypoxia via eIF2}

Formation of the ternary complex requires exchange of eIF2-GDP for GTP and is carried out by the eIF2B exchange factor. This reaction is strongly inhibited by phosphorylation of the eIF $2 \alpha$ subunit at serine 51 , and because eIF2B exists in smaller amounts than eIF2 $\alpha$ (about 20-30\%), small increases in eIF2 $\alpha$ phosphorylation can dramatically suppress translation initiation. We and others have shown that both severe $(<0.05 \%)$ and more moderate $(\sim 1 \%)$ hypoxia cause phosphorylation of eIF $2 \alpha^{12,}{ }^{13}$. In the case of severe hypoxia, this phosphorylation is extremely rapid, occurring within $30 \mathrm{~min}$ of exposure. This effect is fully reversible upon return to oxygenation, and thus not due to depletion of ATP or other nutrients in the media. Under severe hypoxia, eIF $2 \alpha$ phosphorylation levels are highest after short exposures and show a partial recovery after 4-8 hours, consistent with the activation of a negative feedback loop (see below). In contrast, phosphorylation of eIF2 $\alpha$ during moderate hypoxia ( $0.2 \%$ and $1 \%$ oxygen) requires significantly longer hypoxic exposures ( $>6$ hours). Phosphorylation of eIF $2 \alpha$ during hypoxia requires activation of the ER resident kinase PERK, which is known to be involved in the unfolded protein response (UPR).

Figure 1. Polysome analysis of cells grown under aerobic conditions (solid line) or after exposure to 1 hour of hypoxia (dashed line). MRNA and its associated proteins was separated through sedimentation in sucrose gradients. The absorbance as a function of depth is indicated. The first 2 peaks represent free 40S, 605 subunits and the $3^{\text {rd }}$ peak the $80 \mathrm{~s}$ ribosome. Subsequent peaks represent mRNAs that have 2 , or more ribosomes attached and are actively engaged in protein synthesis.

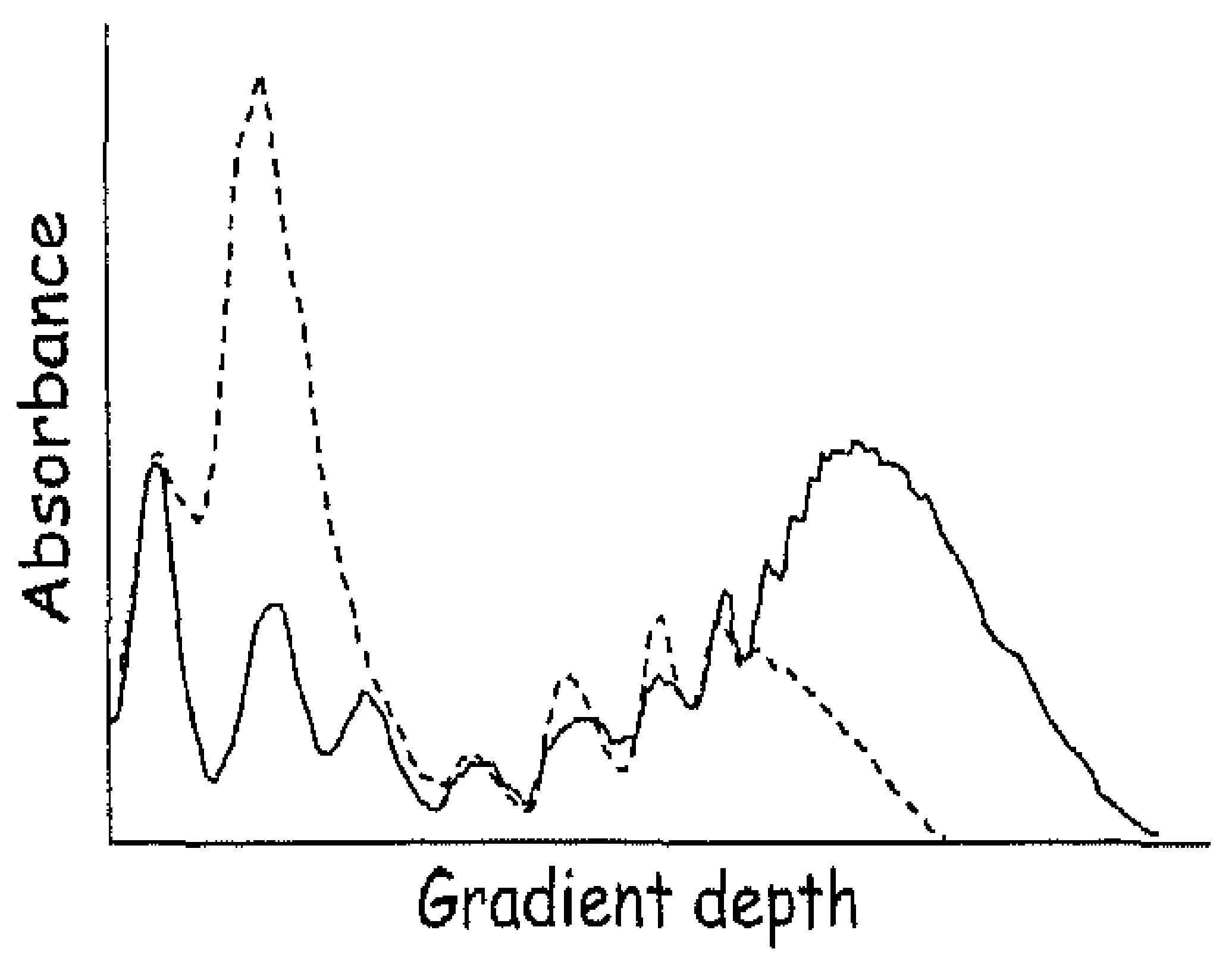


We have examined the translational responses of several cell lines as a function of time during severe $(<0.05 \%)$ hypoxia. This has been carried out primarily by analyzing the levels of ribosome association with mRNA (polysome profiles). Figure 1 shows a typical polysome profile, in which it is possible to estimate both the percentage of ribosomes involved in translation (overall translation) as well as the average number of ribosomes per transcript (ribosome density). We have shown that hypoxia causes a rapid, eIF2 $\alpha$ dependent loss in polysomal mRNA ${ }^{13}$. Overall translation was estimated to drop from over $60 \%$ under normal conditions to $\sim 20 \%$ within 1 hour of hypoxia. Furthermore, the polysomes that remained were shifted to lower molecular weight reflecting a significant drop in ribosome density. This is consistent with a drop in ternary complex availability and a slowing in the recruitment of ribosome complexes to the mRNA. Similar to the phosphorylation of eIF $2 \alpha$, there appeared to be a partial recovery in overall translation after 4-8 hours, and a very large recovery in ribosome density. Cells derived from knock-in mice containing a non-phosphorylatable allele of eIF2 $\alpha$ (S51A) were unable to effectively inhibit overall translation during short hypoxic exposures, and showed no changes in ribosome density at any time during hypoxia. However, these cells displayed similar levels of translational suppression as the wild type cells after 16 hours of hypoxia. Together these data indicate that severe hypoxia elicits a biphasic inhibition of translation. The first phase is due to transient eIF $2 \alpha$ phosphorylation, which blocks ternary complex formation and slows the recruitment of ribosomes onto mRNA transcripts. The second phase occurs more slowly in an eIF $2 \alpha$ independent manner and is capable of reducing overall translation without reducing ribosome density. This second phase of translation inhibition correlates with disruption of the cap binding complex eIF4F (see figure 2).

\section{Translational control during hypoxia via eIF4F}

The availability of the cap-binding protein eIF4E is rate-limiting under normal conditions. The necessity for strict regulation of this protein is 
underlined by the fact that eIF4E is often over expressed in tumors and that it plays a clear role during tumorigenesis ${ }^{17}{ }^{18}$. This regulation is achieved by a family of eIF4E binding proteins (4E-BPs), which sequester eIF4E in their hypophosphorylated form. ${ }^{19}$ Phosphorylation of the 4E-BPS is largely controlled by the mammalian target of rapamycin (mTOR) which integrates signals from several upstream signaling pathways. Hyperphosphorylation of the 4E-BPs lowers the affinity for eIF4E, resulting in an increased interaction between eIF4E and eIF4G and stimulation of translation.

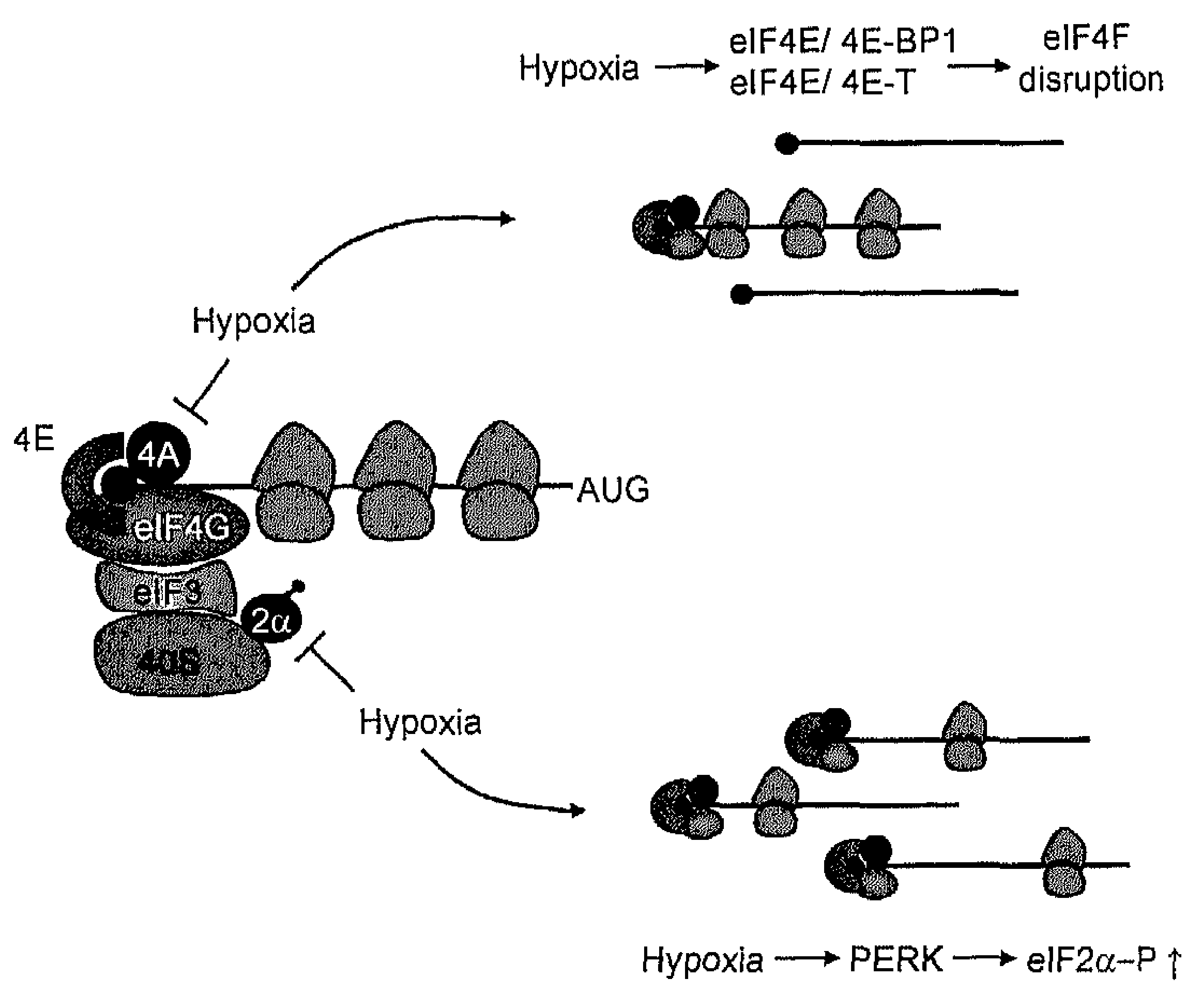

Figure 2. Control of translation initiation during hypoxia is mediated through two distinct mechanisms; (i) eIF4F disruption and (ii) inactivation of the ternary complex. The first is through loss of interaction between eIF4E and eIF4G as a result of increased binding of eIF4E with $4 E-B P 1$ or $4 E-T$. This affects the fraction of $m R N A$ that is involved in translation not the ribosome density on that mRNA. The second mechanism is through PERK dependent phosphorylation of eIF $2 \alpha$. Here the ribosome loading onto the MRNA is impaired due to inactive ternary complex, whereas the fraction of $\mathrm{mRNA}$ involved in translation remains the same. 
A number of publications have suggested that hypoxia can disturb formation of the eIF4F complex through a reduction in mTOR activity and a corresponding decrease in the phosphorylation of 4E-BP1. Moderate hypoxia $(1.5 \%)$ in combination with serum deprivation effectively inhibited the activation of $\mathrm{MTOR}$ and subsequent hypophosphorylation of its substrates $4 \mathrm{E}-\mathrm{BP} 1$ and $56 \mathrm{~K}^{20}$. However, it is less clear to what extent this effect mediates the eIF2 $\alpha$ independent changes in overall translation described above. We found that hypophosphorylation of 4E-BP1 and increased association with eIF4E occurred only after prolonged hypoxia $(16 \mathrm{~h})$, whereas decreased association between eIF4E and eIF4G (indicative of eIF4F disruption) was already reduced after 4 hrs of hypoxia. Similarly, the inhibiton of mTOR during moderate hypoxia $(0.5-1 \%)$ did not cause any immediate changes in overall translation, despite measurable differences in $4 \mathrm{E}-\mathrm{BP}$ phosphorylation ${ }^{21}$. In this study, decreases in protein synthesis under moderate hypoxic conditions did not occur for more than 24 hours. It also appears that growth factor signaling can modulate the ability of mTOR to inhibit translation, since serum deprived cells demonstrate $\sim 30 \%$ inhibition in protein synthesis after 16 hours of moderate hypoxia. Similarly, Kaper reported that severe hypoxia is also less effective at inhibiting protein synthesis in cells that express mutations in upstream regulators of $\mathrm{mTOR}^{22}$. Also consistent with these data, Connolly et al. have shown that inhibition of protein synthesis during hypoxia might strongly depend upon cell transformation. ${ }^{23}$ They showed that hypoxia caused a 4E-BP1 dependent decrease in protein synthesis in untransformed cells, but no decrease in cancer cells. We found that the disruption of eIF4F at 4 hours may also be influenced by the redistribution of eIF4E and the eIF4E translocator (4E-T) into either the nucleus or to recently discovered cytoplasmic bodies of mRNA processing (P-bodies) ${ }^{24,25}$. Translocation of eIF4E into either of these cellular locations makes it unavailable to participate in translation initiation. These data suggest hypoxia induced 
eIF4F disruption may be influenced at least in part by an mTOR/4E-BP1 independent mechanism.

Although changes in mTOR activity may not dramatically influence levels of overall translation during moderate or severe hypoxia, it may still influence the translation of specific gene transcripts (see below). Changes in the regulation of mTOR occur frequently in cancer, and thus its regulation by hypoxia may have important implications for the hypoxic phenotype. The regulation of mTOR activity during hypoxia occurs through at least two independent mechanisms. Although mTOR activity is strongly influenced by growth factor signaling through the PI3K/AKT pathway, this pathway does not seem to be responsible for mTOR inhibition during hypoxia ${ }^{20}, 26,27$. However, a downstream effector of the PI3K/AKT pathway, the tuberous sclerosis complex (TSC), was shown to be essential. This complex functions to inhibit mTOR, and cells lacking TSC1 or TSC2 are unable to downregulate mTOR signaling during hypoxia ${ }^{27}$. Brugarolas et al. showed convincingly that activation of TSC2 and inhibition of mTOR during hypoxia occurred due to the induction of a protein called REDD1 ${ }^{27}$. Transcription of REDD1 is induced in a HIF-1 dependent manner suggesting a role for oxygen sensing in the activation of this pathway. ${ }^{28}$

The second pathway that contributes to inhibition of mTOR during hypoxia also sits upstream of TSC2. Under energy deprived conditions AMPK is activated by LKB1 which results in phosphorylation of TSC2 and subsequent inhibition of $m T^{2} R^{29,30}$. This pathway is thus considered as an energy sensor that can slow protein synthesis under conditions of low ATP. A recent report by Liu et al. showed that AMPK becomes progressively activated during 20 hours of exposure to moderate hypoxia in serum replete conditions ${ }^{21}$. Interestingly, under serum deprived conditions AMPK activation and mTOR inhibition occurred more rapidly (within $30 \mathrm{~min}$ ), although no corresponding effect on overall translation was observed at this time. Unlike REDD1, the activation of AMPK under these conditions 
can explain why inhibition of mTOR occurs independently of HIF activation $^{20,21}$.

\section{Translational mechanisms for selective gene expression during hypoxia}

Upstream open reading frames and ATF4

Although hypoxia causes a global inhibition in translation there are clearly many proteins that affect cell survival and cell behavior that are induced under these conditions. How can such proteins bypass the general inhibition in protein synthesis? The answer to this question lies largely within the $5^{\prime}$ and $3^{\prime}$ untranslated regions (UTR) of their transcripts. Translation efficiency is influenced by both positive and negative regulatory elements within the UTRs, the importance of which is demonstrated by their conservation throughout evolution ${ }^{31}$. Translation is suppressed by the presence of long 5'UTRs with stem loops and other secondary structures that impede scanning of the $43 \mathrm{~S}$ preinitiation complex for the start codon. Translation can also be repressed by the presence of upstream AUG (UAUG) codons and their associated upstream open reading frames (UORFs), and by RNA binding proteins present in either the $5^{\prime}$ or $3^{\prime}$ UTRs ${ }^{32-34}$. Translation initiation occurs primarily at the first $A \cup G$ encountered by the pre-initiation complex, and a UORF can thereby effectively block initiation from the correct ORF. However, several examples have demonstrated that uORFs can also confer selective translation of a specific transcript under certain circumstances. The best mammalian example of this is ATF4 which codes for a transcription factor activated during the UPR following phosphorylation of eIF $2 \alpha^{35,36}$. ATF4 contains two conserved UORFs within its 5'UTR that together ensure that ATF4 protein is made only under conditions where eIF2 $\alpha$ is phosphorylated. Following translation of the first UORF, the ribosome, which normally would dissociate from the mRNA, stays intact and is able to re-initiate at either the second UORF or the ATF-4 ORF. Before this can 
occur the ribosome needs to acquire a new ternary complex containing eIF2-GTP and Met-tRNA. Under normal circumstances where ternary complex availability is high, re-initiation begins at the second UORF. Reinitiation following translation of the second UORF does not occur and thus expression of ATF4 is prevented. However, under conditions where eIF2 $\alpha$ is phosphorylated, ternary complex availability is low, and there is a higher chance that the ribosome will not have acquired a ternary complex by the time it reaches the second UORF. A scanning ribosome that picks up a ternary complex after passing the second UAUG can then initiate at the ATF4 ORF.

Blais and colleagues were the first to demonstrate that hypoxia can stimulate the translation of ATF4 in a PERK dependent manner ${ }^{37}$. Furthermore, they showed that the 5'UTR of ATF4 could confer selective translation of a reporter plasmid. ATF4 has recently been demonstrated to play a critical role in protecting cells against hypoxia and facilitating tumor growth $^{14}$. This is likely due to its ability to activate downstream genes that are important mediators of reactive oxygen stress ${ }^{38}$. By analyzing the distribution of mRNA within polysomes, we also found that ATF4 translation was rapidly stimulated during hypoxia. Furthermore, two of its downstream targets, CHOP and GADD34 which also contain UORFs are also selectively translated in a manner that is dependent on eIF2 $\alpha$ phosphorylation ${ }^{13,14}$.

At the moment it is unclear how many other genes might be subject to selective translation through a similar process during hypoxia. It has been estimated that $10-30 \%$ of genes contain one or more uORFs, and $15-53 \%$ of mRNAs contain upstream start codons ${ }^{39}, 40$. Furthermore, the ability of uORFs to control translation can be regulated by mechanisms other than phosphorylation of eIF $2 \alpha$ phosphorylation. Translation of the Her2 oncogene is also repressed under normal conditions due to an uORF ${ }^{41}$. Mehta et al. recently showed that translation of Her2 is derepressed by so called 'translational deregulation elements' (TDE) in its $3^{\prime} U^{\prime} R^{42}$. These TDEs facilitate binding of HUR and HnRNPC1/C2, proteins that are also 
Translational control of gene expression during hypoxia

required for efficient Her2 translation. This example highlights the fact that UAUGs and UORFs may affect translation through many different mechanisms, some of which are yet to be described.

Internal ribosome entry sites

Other elements in the UTRs can act as positive regulators of translation, including internal ribosome entry sites (IRESs). An IRES consists of an mRNA secondary structure that facilitates direct ribosome binding independent of formation of eIF4F at the cap. This mechanism was originally discovered in viruses as a means to promote translation of viral mRNAs in infected cells that shut-down host protein synthesis through blockade of eIF4F $\mathrm{F}^{43}$. The list of mammalian mRNAs reported to contain IRES activity is growing, and includes a number of hypoxia regulated genes including HIF-1 $\alpha$ (see below), VEGF and BiP ${ }^{44-48}$. Thus, IRES dependent translation might contribute to selective gene expression during hypoxia when eIF4F integrity is disrupted. The ability of an IRES to support translation may also be regulated by various initiation factors and other associated proteins ${ }^{49}$. Consequently IRES function of specific mRNAs can be induced under specific circumstances ${ }^{49}$. An interesting example is the IRES-dependent translation of cat-1, which can be stimulated through phosphorylation of eIF $2 \alpha^{50}$.

\section{5'Terminal oligopyrimidine tract (TOP) sequences}

As described earlier MTOR signaling results in phosphorylation of its two well known substrates S6K and 4E-BP1. S6K phosphorylation promotes translation of a subset of mRNAs that contain terminal oligopyrimidine tract (5'TOP) sequences in their $5^{\prime}$ UTR. This was originally thought to be due to its ability to phosphorylate the ribosomal protein $S 6$, although this recently was shown not to be the case ${ }^{51}$. However, 5 TOP sequences are found in many components of the translational machinery, including all ribosomal proteins, and their translation is greatly stimulated by $\mathrm{mTOR}^{52}$. 


\section{Chapter 3}

Interestingly the $5^{\prime} \mathrm{UTR}$ of HIF-1 $\alpha$ has also been reported to contain these 5'TOP sequences.

\section{Translational control of HIF-1 $\alpha$}

Oncogene and growth factor mediated translation of HIF-1a

HIF-1 $1 \alpha$ is the oxygen sensitive subunit of HIF that together with the stable HIF- $1 \alpha$ subunit regulates the expression of more than 60 putative genes ${ }^{9}$. Its selective degradation under aerobic conditions occurs due to modification of two proline residues in its oxygen dependent degradation (ODD) domain, by one of a family of prolyl hydroxylase enzymes (PHD13). The hydroxylated form of HIF-1 $1 \alpha$ is recognized by $\mathrm{VHL}$, which targets it for ubiquitination and degradation. HIF-1 $\alpha$ levels are under tight control, evidenced by the fact that the PHD enzymes are themselves targets of HIF, leading to the establishment of a negative feedback loop. The importance of HIF expression in cancer is highlighted by the fact that defects in the proteins that control its degradation, for example through VHL loss or PHD deregulation, can lead to cancer ${ }^{53-55}$. Although degradation is the primary means of HIF-1a regulation, like many genes regulated at the protein level, its expression also depends on its rate of synthesis. HIF-1 $\alpha$ expression is increased in response to growth factor signaling through the PI3K/AKT pathway ${ }^{56,57}$. Increased expression of HIF- $1 \alpha$ has been demonstrated for Ras- transformed cells, PTEN defective glioblastoma cells and after over expression of heregulin, the HER2/neu ligand $56,58-60$. Several lines of evidence suggest that these increases in HIF-1 $\alpha$ occur through increased translation (see figure 3 ). Perhaps the strongest evidence comes from the observation that both HIF-1 $\alpha$ and its downstream target VEGF, are overexpressed in hamartomas derived from Peutz-Jeghers syndrome and tuberous sclerosis complex that arise from mutations in LKB1 and TSC1/2 respectively ${ }^{27}$. Mutations in either of these genes lead to deregulation of mTOR and increased rates of translation. Increased HIF-1 $\alpha$ levels are also seen in cells derived from TSC2 knockout 
mice, and this effect is completely blocked by the MTOR inhibitor rapamycin ${ }^{27}$. The increased translation of HIF-1a therefore provides a possible link between mTOR induced cell growth and the need for increased metabolism and angiogenesis as suggested previously ${ }^{61}$. It is not yet clear how HIF-1a synthesis is selectively stimulated in response to mTOR activation, although it is unlikely that this is due to its reported IRES. An IRES allows translation to occur in a cap-independent manner, whereas mTOR signaling stimulates cap-dependent translation by phosphorylating the eIF4E inhibitor 4E-BP1. A recent study by Thomas et al. suggested that the reduction in HIF-1 $\alpha$ translation upon mTOR inhibition with rapamycin is strongly dependent on its $5^{\prime} U T R$ sequence ${ }^{62}$. Cells engineered to express a HIF-1 $\alpha$ construct lacking the $5^{\prime}$ UTR were resistant to rapamycin induced growth inhibition. This preferential translation was hypothesized to occur due to its $5^{\prime}$ TOP sequences.

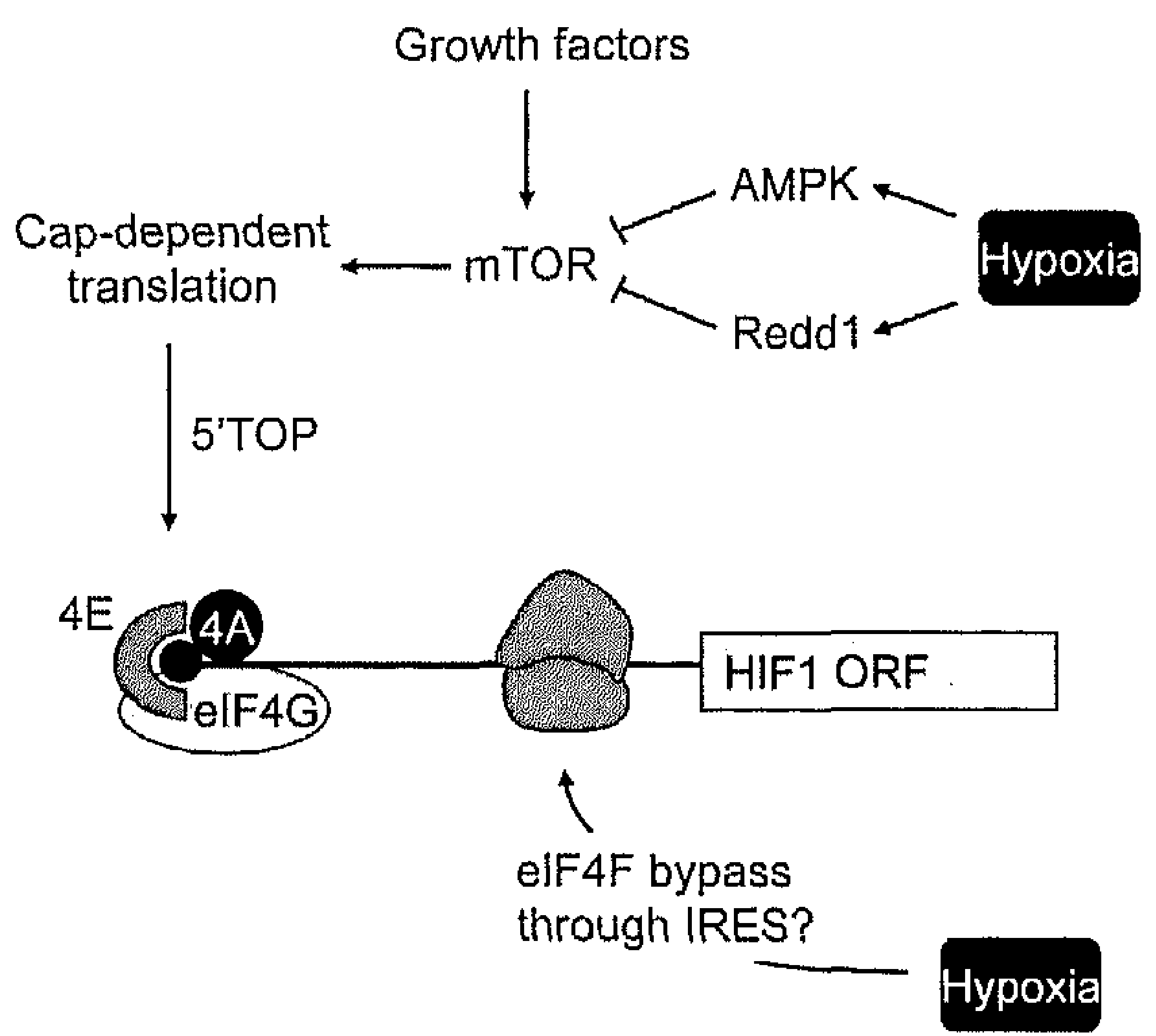

Figure 3. Current understanding of HIF-1 $\alpha$ mRNA translation. Under aerobic conditions HIF-1 $\alpha$ translation can be stimulated through mTOR dependent growth factor signaling. This appears to be mediated by 5 'TOP motives within the 5'UTR of HIF1. During hypoxic exposure mTOR signaling and therefore cap-dependent translation is inhibited. It is thought that hypoxia can promote IRES-dependent translation of HIFI which eliminates the need for eIF4F.

\section{Translation of HIF-1 $\alpha$ during hypoxia}

The fact that HIF-1 $1 \alpha$ translation is sensitive to mTOR inhibition suggests that hypoxia, which inhibits mTOR, may paradoxically also inhibit HIF-1a synthesis. In contrast to this expectation HIF-1 $\alpha$ mRNA is preferentially 
translated during hypoxia and thus able to 'escape' the effects of hypoxia on mTOR as well as the overall reduction in mRNA translation ${ }^{45,63}$. Lang et al. originally showed that this effect was due IRES activity in the 5'UTR of murine HIF- $1 \alpha^{45}$. However, there remains a great deal of controversy over the authenticity of the HIF-1 $\alpha$ IRES as well as several other mammalian mRNAs reported to have IRES activity ${ }^{64,65}$. Furthermore, the group that originally identified the murine HIF-1a IRES recently reported that its apparent activity is largely caused by cryptic promoter activity that is stimulated by the SV40 enhancer element in their reporter plasmid ${ }^{65}$. Using RNA transfection experiments the authors showed that the HIF-1 $\alpha$ $5^{\prime}$ UTR does support a small amount of IRES translation but this activity is nowhere near to that reported previously. However, it has also been suggested that efficient IRES activity in HIF- $1 \alpha$ may require association of other cellular proteins with specific sequences in the HIF-1 $\alpha 5^{\prime}$ UTR. The polypyrimidine tract binding (PTB) protein was shown to stimulate translation of HIF- $1 \alpha$ by binding to its $5^{\prime}$ TOP sequences and promoting IRES activity ${ }^{65}$. Clearly, several questions remain regarding the mechanism of selective HIF-1 $\alpha$ mRNA translation.

The selected examples above demonstrate that our understanding of the effect of translational regulation during hypoxia on gene expression is still very limited. The ability of translation to regulate individual gene expression on a genome wide scale has been investigated in only a few circumstances ${ }^{43,67-70}$. In these studies changes in the levels of efficiently translated (polysomal) mRNA were evaluated using microarray technology and compared to transcriptional (total mRNA) changes. Although limited, these studies have shown that subtle changes in overall translation can influence gene expression in important ways. For example, it was shown that AKT activation causes large changes in the levels of genes found in polysomes at time points that precede changes in mRNA transcription and without significant changes in overall translation ${ }^{70}$.

Several similar studies have begun to assess the contribution of translational regulation during hypoxia on a genome wide scale. Using 


\section{Translational control of gene expression during hypoxia}

microarray analysis Blais et al. identified several genes that displayed an increased translation efficiency during hypoxia, including ATF4 as described above ${ }^{37}$. This study was performed using polysome mRNA from Hela cells isolated after 16 hours of exposure to severe hypoxia and thus translation inhibition at this time point is expected to be dominated by inhibition of eIF4F. We performed a similar study after only 4 hours of hypoxia in which we expected to observe changes mediated by both eIF $2 \alpha$ and eIF4F. We were able to show that of the top 50 genes with the largest induction during hypoxia, $20 \%$ displayed a selective ability to be translated ${ }^{71}$. This included several genes dependent on HIF-1 or involved in the UPR. A genome-wide analysis of translation in Arabidopsis during hypoxia also revealed distinct clusters of genes that show a preferential ability to maintain translation ${ }^{72}$. These genes were involved in abiotic and biotic stress responses and were characterized by having a low GC content in their $5^{\prime}$ UTRs. Analysis of polysomal RNA in various cell lines under different levels and durations of hypoxia will also play an important role elucidating $5^{\prime}$ and $3^{\prime}$ UTR translational control elements that mediate preferential translation of various genes. Studies in genetic models which lack the ability to control translation through either eIF $2 \alpha$ or eIF4F will also help to clarify the relative importance of these two pathways on gene expression during hypoxia.

\section{Concluding remarks}

Translational control through both UPR dependent and independent mechanisms has emerged as an important regulatory process in the response to hypoxia that affects both hypoxic cell tolerance and the hypoxic phenotype. Changes in mRNA translation affect the key hypoxia regulated transcription factors ATF4 and HIF, and thus indirectly influence the expression of many other genes. Although the pathways that direct translational control during hypoxia have become partly elucidated, several questions remain for future investigation. One challenge will be to 
elucidate the exact mechanisms through which genes like HIF-1 $\alpha$ are preferentially translated during hypoxia. Understanding of this process might be important in the design of more effective anti-tumor treatments. Interestingly, targeting HIF- $1 \alpha$ translation is already an active area of research. In a screen for compounds able of block HIF activity, Rapisarda et al. identified captothecin, a topo 1 inhibitor ${ }^{73}$. They subsequently showed that its ability to inhibit HIF-1 $\alpha$ occurred through suppression of its translation. Similarly, the anti-cancer agents taxol and 2ME2, both which disrupt microtubules, have also been suggested to block HIF-1 $\alpha$ through a translational mechanism ${ }^{74}$. It is also important to keep in mind that inhibition of growth factor signaling, and thus PI3K/AKT-mTOR signaling, can also suppress HIF activation by inhibiting its translation. This effect was recently demonstrated with tyrosine kinase inhibitors to EGFR $^{75}$.

A second important area that has not been adequately addressed is the degree to which control of mRNA translation during hypoxia may be altered in cancer. Control of mRNA translation via disruption of eIF4F is indeed expected to vary considerably amongst different tumors since the upstream pathways that control the assembly of this complex are frequently disrupted in cancer. eIF4F has emerged as an important target of PI3K signaling during tumor development. It will thus be important to assess the differences in regulation of translation and gene expression during hypoxia in cells with specific defects in these upstream signaling pathways. Furthermore, although eIF4F assembly under hypoxia is disrupted, the importance of this effect on tumor growth or hypoxia tolerance has yet to be addressed.

\section{Acknowledgements}

We would like to thank all members of the Maastro labs whose work has been described in this review. We also acknowledge our many collaborators that contributed to this work. This work was financially 
supported the Netherlands Organization for Scientific Research (NWO), the Dutch Cancer Society (KWF Kankerbestrijding) and the Euroxy grant from the 6th framework of the EU to B.G.W.

\section{References}

1. Brown JM, Wilson WR: Exploiting tumour hypoxia in cancer treatment, Nat Rev Cancer 2004, 4:437-447

2. Lanzen J, Braun RD, Klitzman B, Brizel D, Secomb TW, Dewhirst MW: Direct demonstration of instabilities in oxygen concentrations within the extravascular compartment of an experimental tumor, Cancer Res 2006, 66:2219-2223

3. Teicher BA: Hypoxia and drug resistance, Cancer Metastasis Rev 1994, 13:139-168

4. Teicher BA: Physiologic mechanisms of therapeutic resistance. Blood flow and hypoxia, Hematol Oncol Clin North Am 1995, 9:475-506

5. Hockel $M$, Schlenger $K$, Aral $B$, Mitze $M$, Schaffer $U$, Vaupel $P$ : Association between tumor hypoxia and malignant progression in advanced cancer of the uterine cervix, Cancer Res 1996, 56:4509-4515

6. Airley R, Loncaster J, Davidson S, Bromley M, Roberts S, Patterson A, Hunter $R$, Stratford I, West C: Glucose transporter glut-1 expression correlates with tumor hypoxia and predicts metastasis-free survival in advanced carcinoma of the cervix, Clin Cancer Res 2001, 7:928-934

7. Hockel M, Vaupel P: Biological consequences of tumor hypoxia, Semin Oncol 2001, 28:36-41.

8. Graeber TG, Osmanian C, Jacks T, Housman DE, Koch CJ, Lowe SW, Giaccia AJ: Hypoxia-mediated selection of cells with diminished apoptotic potential in solid tumours, Nature 1996, 379:88-91

9. Semenza GL: Targeting HIF-1 for cancer therapy, Nat Rev Cancer 2003, 3:721-732

10. Pettersen EO, Juul NO, Ronning OW: Regulation of protein metabolism of human cells during and after acute hypoxia, Cancer Res 1986, 46:4346-4351

11. Kraggerud SM, Sandvik JA, Pettersen EO: Regulation of protein synthesis in human cells exposed to extreme hypoxia, Anticancer Res 1995, 15:683-686

12. Koumenis C, Naczki C, Koritzinsky M, Rastani S, Diehl A, Sonenberg $N$, Koromilas A, Wouters BG: Regulation of protein synthesis by hypoxia via activation of the endoplasmic reticulum kinase PERK and phosphorylation of the translation initiation factor eIF2alpha, Mol Cell Biol 2002, 22:7405-7416

13. Koritzinsky $M$, Magagnin MG, van den Beucken $T$, Seigneuric $R$, Savelkouls $K$, Dostie J, Pyronnet S, Kaufman RJ, Weppler SA, Voncken JW, Lambin P, Koumenis $C_{r}$ Sonenberg N, Wouters BG: Gene expression during acute and prolonged hypoxia is regulated by distinct mechanisms of translational control, Embo J 2006, 25:1114-1125

14. Bi M, Naczki C, Koritzinsky M, Fels D, Blais J, Hu N, Harding $H$, Novoa I, Varia $M$, Raleigh J, Scheuner D, Kaufman RJ, Bell J, Ron D, Wouters BG, Koumenis C: ER stress-regulated translation increases tolerance to extreme hypoxia and promotes tumor growth, Embo ] 2005, 24:3470-3481 
15. Gingras $A C$, Raught $B$, Sonenterg $N$ : eIFa intiation factors: effectors of mRNA recruitment to rbosomes and regulators of transiation. Annu Rev Biochem $1999,68: 913-963$

16. Holck $M$, Sonenberg $N$ : Translational control in stress and apoptosis, Nat Rev Mol Cell Biol 2005, 6:318-327

17. Ruggero D, Montanaro L, Ma L, Xu W, Londei P. Cordon-Cardo C, Pandolf, PP: The translation factor elF-4E promotes tumor formation and cooperates with C-Myc in iymphomagenesis, Nat Med 2004, 10:484-486

18. Wendel $H G$, De Stanchina $E$, Fridman 1S, Malina A, Ray $S$, Kogan $S$, Cordon. Cardo C, Pelletier J, Lowe SW: Survival signalling by Akt and elfaE in oncogenesis and cancer therapy, Nature 2004, 428:332.337

19. Pause A, Belsham GJ, Gingras AC, Donze O, Lin TA, Lawrence JC, Jr. Sonenberg $N$ : Insulin-dependent stimulation of protein synthesis by phosphorylation of a regulator of 5' Cap function, Nature 1994, 371:762-767

20. Arsham AM, Howell Jj, Simon MC: A Novel Hypoxia-inducible Factorindependent Hypoxic Response Regulating Mammalian Target of Rapamyein and Its Targets, 3. Biol. Chem. 2003, 278:29655-29660

21. Liu L, Cash TP, Jones RG, Keith B, Thompson CB, Simon MC: Hypoxia-induced energy stress regulates mRNA translation and cell growth, Mol Cell 2006, $21: 521.531$

22. Kaper F, Dornhoefer $N$, Giaccia AJ: Mutations in the PI3K/PTEN/TSC2 pathway contribute to mammalian target of rapamycin activity and increased translation under hypoxic conditions, Cancer Res 2006, 66:1561-1569

23. Connolly $E$, Braunstein $S$, Formenti $S$, Schneider RJ: Hypoxia inhibits protein synthesis through a 4E-BP1 and elongation factor 2 kinase pathway controlled by mTOR and uncoupled in breast cancer cells, Mol Cell Biol 2006, 26:3955* 3965

24. Dostie J, Ferraiuolo M, Pause A, Adam SA, Sonenberg N: A novel shuttling protein, 4E-T, mediates the nuclear import of the MRNA $5^{2}$ cap-binding protein, elF4E, Embo J 2000, 19:3142-3156

25. Andrei $M A$, Ingelfinger $D$, Heintzmann $R$, Achsel $T$, Rivera-Pomar $R$, Luhrmann $R$ : A role for eIF4E and eIF4E-transporter in targeting mRNPs to mammalian processing bodies, Rna 2005, 11:717-727

26. Cai SL, Tee AR, Short JD, Bergeron JM, Kim J, Shen J, Guo R, Johnson CL, Kiguchi K, Walker CL: Activity of TSC2 is inhibited by AKT-mediated phosphorylation and membrane partitioning, J Cell Biol 2006, 173:279-289

27. Brugarolas J, Lei $K$, Hurley RL, Manning BD, Reiling JH, Hafen E, Witters LA, Ellisen LW, Kaelin WG, Jr,: Regulation of mTOR function in response to hypoxia by REDD1 and the TSC1/TSC2 tumor suppressor complex, Genes Dev 2004, 18:2893-2904

28. Schwarzer R, Tondera D, Arnold W, Giese K, Klippel A, Kaufmann J: REDD1 integrates hypoxia-mediated survival signaling downstream of phosphatidylinositol 3-kinase, Oncogene 2005, 24:1138-1149

29. Corradetti MN, Inokı K, Bardeesy N, DePinho RA, Guan KL: Regulation of the TSC pathway by LKB1: evidence of a molecular link between tuberous sclerosis complex and Peutz-Jeghers syndrome, Genes Dev 2004, 18:15331538

30. Shaw RJ, Bardeesy N, Manning BD, Lopez L, Kosmatka M, DePinho RA, Cantley LC: The LKB1 tumor suppressor negatively regulates mTOR signaling, Cancer Cell 2004, 6:91-99

31. Spicher A, Guicherit OM, Duret L, Aslanian A, Sanjines EM, Denko NC, Giaccia AJ, Blau HM: Highly conserved RNA sequences that are sensors of environmental stress, Mol Cell Biol 1998, 18:7371-7382 
Translational control of gene expression during hypoxia

32. Kuersten S, Goodwin EB: The power of the $3^{\prime}$ UTR: translational control and development, Nat Rev Genet 2003, 4:626-637

33. Meijer HA, Thomas AA: Control of eukaryotic protein synthesis by upstream open reading frames in the 5 '-untranslated region of an mRNA, Biochem J 2002, 367:1-11

34. Sachs MS, Geballe AP: Downstream control of upstream open reading frames, Genes Dev 2006, 20:915-921

35. Lu PD, Harding HP, Ron D: Translation reinitiation at alternative open reading frames regulates gene expression in an integrated stress response, J Cell Biol 2004, 167:27-33

36. Vattem KM, Wek RC: Reinitiation involving upstream ORFs regulates ATF4 mRNA translation in mammalian cells, Proc Natl Acad Sci U S A 2004, 101:11269-11274

37. Blais JD, Filipenko V, Bi M, Harding HP, Ron D, Koumenis C, Wouters BG, Bell JC: Activating transcription factor 4 is translationally regulated by hypoxic stress, Mol Cell Biol 2004, 24:7469-7482

38. Harding HP, Zhang $Y$, Zeng $H$, Novoa I, Lu PD, Calfon M, Sadri N, Yun C, Popko B, Paules R, Stojdl DF, Bell JC, Hettmann T, Leiden JM, Ron D: An integrated stress response regulates amino acid metabolism and resistance to oxidative stress, Mol Cell 2003, 11:619-633

39. Churbanov A, Rogozin IB, Babenko VN, Ali H, Koonin EV: Evolutionary conservation suggests a regulatory function of AUG triplets in 5'-UTRs of eukaryotic genes, Nucleic Acids Res 2005, 33:5512-5520

40. Suzuki $Y$, Ishihara $D$, Sasaki $M$, Nakagawa $H$, Hata $H$, Tsunoda $T$, Watanabe M, Komatsu T, Ota T, Isogai T, Suyama A, Sugano S: Statistical analysis of the $5^{\prime}$ untranslated region of human mRNA using "Oligo-Capped" cDNA libraries, Genomics 2000, 64:286-297

41. Child SJ, Miller MK, Geballe AP: Translational control by an upstream open reading frame in the HER-2/neu transcript, J Biol Chem 1999, 274:2433524341

42. Mehta A, Trotta CR, Peltz SW: Derepression of the Her-2 UORF is mediated by a novel post-transcriptional control mechanism in cancer cells, Genes Dev $2006,20: 939-953$

43. Johannes G, Carter MS, Eisen MB, Brown PO, Sarnow P: Identification of eukaryotic mRNAs that are translated at reduced cap binding complex eIF4F concentrations using a cDNA microarray, Proc Natl Acad Sci U S A 1999, 96:13118-13123

44. Bonnal S, Boutonnet C, Prado-Lourenco L, Vagner S: IRESdb: the Internal Ribosome Entry Site database, Nucleic Acids Res 2003, 31:427-428

45. Lang KJ, Kappel A, Goodall GJ: Hypoxia-inducible factor-1alpha mRNA contains an internal ribosome entry site that allows efficient translation during normoxia and hypoxia, Mol Biol Cell 2002, 13:1792-1801

46. Stein I, Itin A, Einat P, Skaliter R, Grossman Z, Keshet E: Translation of vascular endothelial growth factor mRNA by internal ribosome entry: implications for translation under hypoxia, Mol Cell Biol 1998, 18:3112-3119

47. Macejak DG, Sarnow P: Internal initiation of translation mediated by the 5 leader of a cellular mRNA, Nature 1991, 353:90-94

48. Thoma $C_{r}$ Bergamini G, Galy B, Hundsdoerfer P, Hentze MW: Enhancement of IRES-mediated translation of the $c-m y c$ and BiP mRNAs by the poly $(A)$ tail is independent of intact eIF4G and PABP, Mol Cell 2004, 15:925-935

49. Hellen CU, Sarnow P: Internal ribosome entry sites in eukaryotic mRNA molecules, Genes Dev 2001, 15:1593-1612 
50. Yaman I, Fernandez J, Liu H, Caprara M, Komar AA, Koromilas AE, Zhou L, Snider MD, Scheuner D, Kaufman RJ, Hatzoglou M: The zipper model of translational control: a small upstream ORF is the switch that controls structural remodeling of an mRNA leader, Cell 2003, 113:519-531

51. Ruvinsky I, Meyuhas O: Ribosomal protein $\mathbf{S 6}$ phosphorylation: from protein synthesis to cell size, Trends Biochem Sci 2006,

52. Jefferies HB, Fumagalli $S$, Dennis PB, Reinhard C, Pearson RB, Thomas G: Rapamycin suppresses 5'TOP mRNA transiation through inhibition of p70s6k, Embo J 1997, 16:3693-3704

53. Maxwell PH, Wiesener MS, Chang GW, Clifford SC, Vaux EC, Cockman ME, Wykoff CC, Pugh CW, Maher ER, Ratcliffe PJ: The tumour suppressor protein VHL targets hypoxia-inducible factors for oxygen-dependent proteolysis, Nature 1999, 399:271-275

54. Stebbins CE, Kaelin WG, Jr., Pavletich NP: Structure of the VHL-ElonginCElonginB complex: implications for VHL tumor suppressor function, Science 1999, 284:455-461

55. Selak MA, Armour SM, MacKenzie ED, Boulahbel $H$, Watson DG, Mansfield KD, Pan $Y$, Simon MC, Thompson CB, Gottlieb E: Succinate links TCA cycle dysfunction to oncogenesis by inhibiting HIF-alpha prolyl hydroxylase, Cancer Cell 2005, 7:77-85

56. Mazure NM, Chen EY, Laderoute KR, Giaccia AJ: Induction of vascular endothelial growth factor by hypoxia is modulated by a phosphatidylinositol 3kinase/Akt signaling pathway in Ha-ras-transformed cells through a hypoxia inducible factor-1 transcriptional element, Blood 1997, 90:3322-3331

57. Zhong $H$, Chiles $K$, Feldser D, Laughner $E$, Hanrahan $C$, Georgescu MM, Simons JW, Semenza GL: Modulation of hypoxia-inducible factor 1alpha expression by the epidermal growth factor/phosphatidylinositol 3kinase/PTEN/AKT/FRAP pathway in human prostate cancer cells: implications for tumor angiogenesis and therapeutics, Cancer Res 2000, 60:1541-1545

58. Zhong $H$, De Marzo AM, Laughner $E$, Lim M, Hilton DA, Zagzag D, Buechler $P$, Isaacs WB, Semenza GL, Simons JW: Overexpression of hypoxia-inducible factor 1alpha in common human cancers and their metastases, Cancer Res $1999,59: 5830-5835$

59. Zundel W, Schindler C, Haas-Kogan D, Koong A, Kaper F, Chen E, Gottschalk $A R$, Ryan $H E$, Johnson RS, Jefferson $A B$, Stokoe $D$, Giaccia AJ: Loss of PTEN facilitates HIF-1-mediated gene expression, Genes Dev 2000, 14:391-396

60. Laughner $E$, Taghavi P, Chiles K, Mahon PC, Semenza GL: HER2 (neu) signaling increases the rate of hypoxia-inducible factor 1alpha (HIF-1alpha) synthesis: novel mechanism for HIF-1-mediated vascular endothelial growth factor expression, Mol Cell Biol 2001, 21:3995-4004

61. Brugarolas J, Kaelin WG, Jr.: Dysregulation of HIF and VEGF is a unifying feature of the familial hamartoma syndromes, Cancer Cell 2004, 6:7-10

62. Thomas GV, Tran C, Mellinghoff IK, Welsbie DS, Chan E, Fueger B, Czernin J, Sawyers CL: Hypoxia-inducible factor determines sensitivity to inhibitors of mTOR in kidney cancer, Nat Med 2006, 12:122-127

63. Gorlach A, Camenisch G, Kvietikova I, Vogt L, Wenger RH, Gassmann M: Efficient translation of mouse hypoxia-inducible factor-1alpha under normoxic and hypoxic conditions, Biochim Biophys Acta 2000, 1493:125-134

64. Kozak M: A second look at cellular mRNA sequences said to function as internal ribosome entry sites, Nucleic Acids Res 2005, 33:6593-6602

65. Bert AG, Grepin R, Vadas MA, Goodall GJ: Assessing IRES activity in the HIF1 alpha\} and other cellular 5' UTRs, Rna 2006, 12:1074-1083 
66. Schepens B, Tinton SA, Bruynooghe $Y$, Beyaert $R$, Cornelis S: The polypyrimidine tract-binding protein stimulates HIF-1alpha IRES-mediated translation during hypoxia, Nucleic Acids Res 2005, 33:6884-6894

67. Richter JD: Translational control in development: a perspective, Dev Genet $1993,14: 407-411$

68. Mikulits W, Pradet-Balade B, Habermann B, Beug H, Garcia-Sanz JA, Mullner EW: Isolation of translationally controlled mRNAs by differential screening, Faseb J 2000, 14:1641-1652

69. Galban S, Fan J, Martindale JL, Cheadle C, Hoffman B, Woods MP, Temeles G, Brieger J, Decker J, Gorospe $M$ : von Hippel-Lindau protein-mediated repression of tumor necrosis factor alpha translation revealed through use of cDNA arrays, Mol Cell Biol 2003, 23:2316-2328

70. Rajasekhar VK, Viale A, Socci ND, Wiedmann M, Hu X, Holland EC: Oncogenic Ras and Akt signaling contribute to glioblastoma formation by differential recruitment of existing mRNAs to polysomes, Mol Cell 2003, 12:889-901

71. Koritzinsky $M$, Seigneuric $R$, Magagnin $M G$, van den Beucken $T$, Lambin $P$, Wouters $B G$ : The hypoxic proteome is influenced by gene-specific changes in mRNA translation, Radiother Oncol 2005, 76:177-186

72. Branco-Price C, Kawaguchi R, Ferreira RB, Bailey-Serres J: Genome-wide analysis of transcript abundance and translation in Arabidopsis seedlings subjected to oxygen deprivation, Ann Bot (Lond) 2005, 96:647-660

73. Rapisarda A, Uranchimeg B, Sordet O, Pommier Y, Shoemaker RH, Melitlo G: Topoisomerase I-mediated inhibition of hypoxia-inducible factor 1: mechanism and therapeutic implications, Cancer Res 2004, 64:1475-1482

74. Mabjeesh NJ, Escuin D, LaVallee TM, Pribluda VS, Swartz GM, Johnson MS, Willard MT, Zhong $H$, Simons JW, Giannakakou P: 2ME2 inhibits tumor growth and angiogenesis by disrupting microtubules and dysregulating HIF, Cancer Cell 2003, 3:363-375

75. Pore N, Jiang Z, Gupta A, Cerniglia G, Kao GD, Maity A: EGFR tyrosine kinase inhibitors decrease VEGF expression by both hypoxia-inducible factor (HIF)-1independent and HIF-1-dependent mechanisms, Cancer Res 2006, 66:31973204 


\section{CHAPTER 4}

\section{The hypoxic proteome is influenced}

by gene-specific changes in MRNA translation
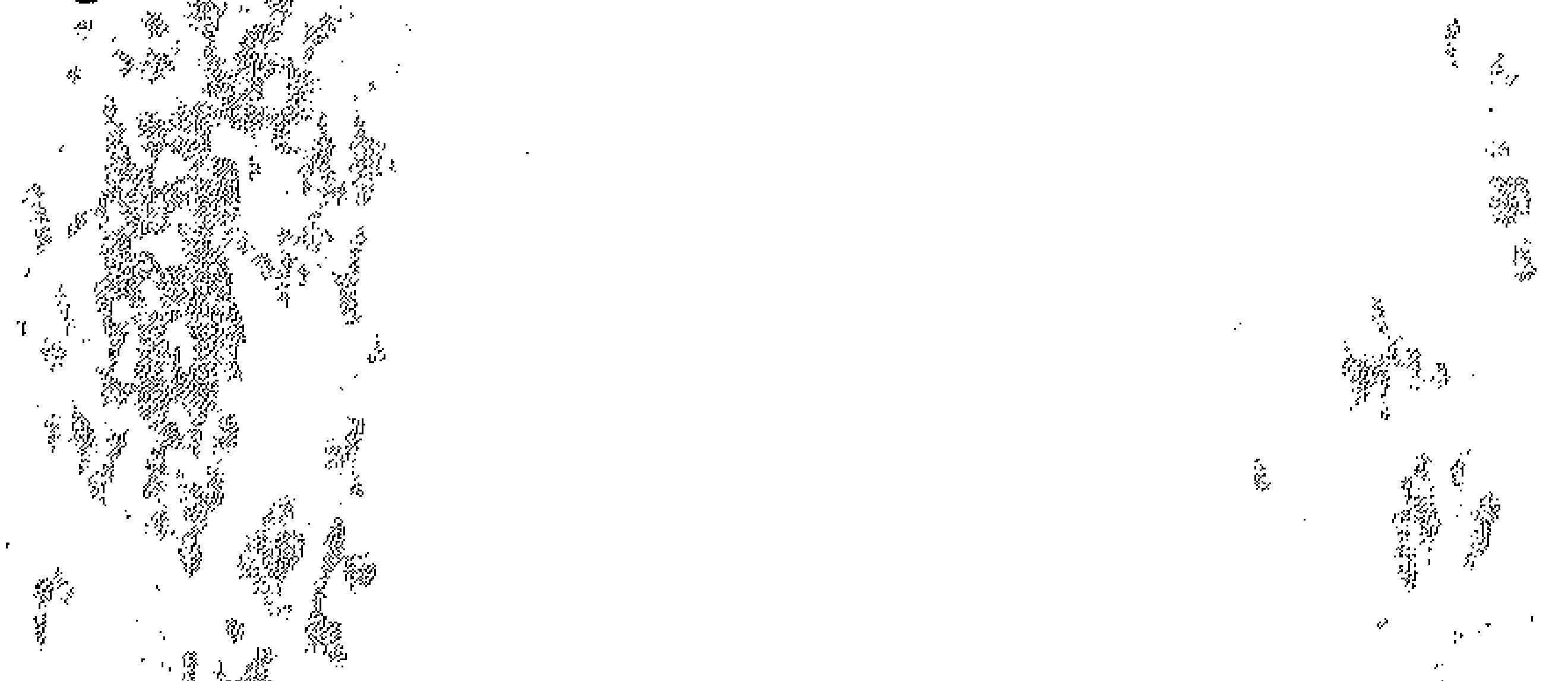

Radiother Oncol, 2005 Aug; 76(2):177-186,

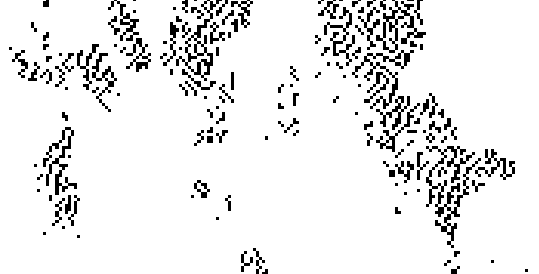

Koritzinsky M, Seigneuric R, Magagnin MG, van den Beucken $T$, Lambin P, Wolvers BG. The hypoxic proteome is influenced by gene-specific changes in mRNA translation. 


\begin{abstract}
Background and purpose: Hypoxia causes a rapid reduction in mRNA translation efficiency. This inhibition does not affect all mRNA species to the same extent and can therefore contribute significantly to hypoxiainduced differential protein expression. Our aim in this study was to characterize changes in gene expression during acute hypoxia and evaluate the contribution of regulation via mRNA translation on these changes. For each gene, the contribution of changes in mRNA abundance versus mRNA translation was determined.

Materials and Methods: DU145 prostate carcinoma cells were exposed to 4 hours of hypoxia $\left(<0.02 \% \mathrm{O}_{2}\right)$. Efficiently translated mRNAs were isolated by sedimentation through a sucrose gradient. Affymetrix microarray technology was used to evaluate both the transcriptional and translational contribution to gene expression. Results were validated by quantitative PCR.

Results: 120 genes were more than 4-fold upregulated by hypoxia in the efficiently translated fraction of mRNA, in comparison to only 76 genes at the level of transcription. Of the 50 genes demonstrating the largest changes in translation, 11 were found to be more than 2-fold over represented in the translated fraction in comparison to their overall transcriptional level. The gene with the highest translational contribution to its induction was CITED-2, which is a negative regulator of HIF-1 transcriptional activity.

Conclusions: Gene-specific regulation of mRNA translation contributes significantly to differential gene expression during hypoxia.
\end{abstract}




\section{Introduction}

The presence of hypoxic (poorly oxygenated) areas in human solid tumors is well documented ${ }^{1-9}$. Hypoxia causes radiation resistance and limits radiotherapy efficacy ${ }^{2-4}, 10,11$. Tumor hypoxia has also been associated with a lower susceptibility to apoptosis ${ }^{12,13}$, increased angiogenesis ${ }^{14-17}$ as well as higher metastatic and invasive potential2, 10, 18-20. The cellular response to hypoxia which underlies its negative impact on tumor biology is believed to be due in large part to dynamic changes in gene expression.

$A$ vast number of hypoxia-induced changes in gene expression have been described. There has been particular focus on changes mediated through the hypoxia inducible transcription factor HIF-1. HIF-1 promotes transcription of its target genes through binding a conserved hypoxia responsive element (HRE) gene promoter together with its co activator $\mathrm{CBP} / \mathrm{p300}$. More than 60 putative downstream target genes have been identified (for a review $\mathrm{se}^{21}$ ), including genes involved in glycolysis (e.g. PGK-1) ${ }^{22}$, angiogenesis (e.g. VEGF) ${ }^{23,24}, \mathrm{pH}$ regulation (CA9) and survival (e.g. EPO) ${ }^{25,} 26$. HIF-1 is activated during hypoxia through protein stabilization of its subunit HIF- $1 \alpha$, which is targeted for ubiquitin mediated degradation by $\mathrm{pVHL}$ (von-Hippel Lindau) during normoxia ${ }^{27}$.

Although the transcriptional response to hypoxia is clearly very important, cells in normal and cancerous tissue often experience short transient exposures to hypoxia which do not allow sufficient time for a productive transcriptional response ${ }^{28-30}$. However, a biological response to acute hypoxia is presumably required to maintain energy homeostasis and provide adaptation to the quickly changing redox microenvironment. A potentially important point for regulating gene expression that is able to respond rapidly to changes in the microenvironment is the process of mRNA translation (protein synthesis). Indeed, it has been shown that overall mRNA translation is severely but reversibly inhibited during hypoxic conditions ${ }^{13}, 31,32$, and this inhibition can be detected after only a few minutes of oxygen deprivation. The hypoxia-induced inhibition in mRNA translation is at least in part mediated through PERK-dependent 
phosphorylation of the translation initiation factor eIF2 $\alpha^{31}$. PERK is known to be activated in response to endoplasmic reticulum (ER) stress suggesting a possible mechanism through which oxygen activates this pathway.

Protein synthesis is the highest cellular consumer of ATP ${ }^{33,34}$, and thus its inhibition during hypoxia probably serves to maintain energy homeostasis. However, it is important to realize that the inhibition of global mRNA translation potentially affects different mRNA species (different genes) to highly varying degrees. This variation is based on the fact that mRNAs from different genes have different dependencies and/or affinities for the cellular translation machinery ${ }^{35,36}$. Thus while some mRNAs may be completely dependent on the translation factors that are inhibited during hypoxia, others can remain translated through alternative mechanisms and thereby bypass the overall translation block. For example, the transcription factor ATF-4 is translationally induced by ER stress in spite of PERK-dependent eIF2 $\alpha$ phosphorylation ${ }^{35}$, and ATF-4 in turn causes transcriptional activation of a conserved gene expression program called the unfolded protein response (UPR) (reviewed $\mathrm{in}^{37}$ ). Hence, the inhibition of overall translation is a potentially powerful cellular tool for rapidly changing gene expression during hypoxia.

In this study, our aim was to evaluate the translational contribution to differential gene expression during acute hypoxia. Utilizing Affymetrix microarray technology we here show that there are significant changes in gene-specific mRNA translation efficiency during hypoxia.

\section{Materials and Methods}

\section{Cell culture and hypoxic conditions}

Exponentially growing cervical (HeLa) and prostate (DU145) carcinoma cells were seeded on glass dishes in DMEM or McCoy media with $10 \%$ foetal calf serum (FCS) (Sigma-Aldrich) and transferred to a hypoxic culture chamber (MACS VA500 microaerophilic workstation, Don Whitley 
Scientific, Shipley, UK). The atmosphere in the chamber consisted of $5 \%$ $\mathrm{H}_{2}, 5 \% \mathrm{CO}_{2}$, the desired concentration of $\mathrm{O}_{2}$ and residual $\mathrm{N}_{2}$. An anoxic atmosphere was ensured by the inclusion of a catalyst in the hypoxic chamber that catalysed the conversion of $\mathrm{H}_{2}$ with any $\mathrm{O}_{2}$ to $\mathrm{H}_{2} \mathrm{O}$.

\section{D gel electrophoresis}

Cells were washed twice with ice-cold $10 \mathrm{mM}$ Tris/250 mM sucrose buffer $\mathrm{pH} 7.0$ and lysed in a $7 \mathrm{M}$ urea/2 $\mathrm{M}$ thiourea based lysisbuffer. The samples were applied to $24 \mathrm{~cm}$ pH 3-10 NL IPG strips (Amersham Biosciences). Following isoelectric focusing, the strips were placed on the Protean Plus Dodeca Cell (Bio-Rad Laboratories) to perform a second dimension separation using 12\% SDS-PAGE gels. The gels were stained with SYPRO Ruby dye (Bio-Rad Laboratories) and imaged on a Molecular Imager FX (Bio-Rad Laboratories).

\section{Polysomal fractionation and analysis}

Cells were treated with $0.1 \mathrm{mg} / \mathrm{ml}$ cycloheximide $(\mathrm{CHX})$ for $3 \mathrm{~min}$ at $37^{\circ} \mathrm{C}$, washed twice with ice cold PBS/CHX and harvested by scraping in lysis buffer ( $1 \%$ Triton X-100, $0.3 \mathrm{M} \mathrm{NaCl}, 15 \mathrm{mM} \mathrm{MgCl} 2,15 \mathrm{mM}$ Tris ( $\mathrm{pH} 7.4$ ), $0.1 \mathrm{mg} / \mathrm{ml} \mathrm{CHX}, 100$ units RNAse-In (Ambion)) at $4{ }^{\circ} \mathrm{C}$. Nuclei were removed, 200ug/ml heparin was added and residual debris was removed by centrifugation. The lysate was layered on a $10 \mathrm{ml}$ continuous sucrose gradient $(20-50 \%$ sucrose in $15 \mathrm{mM} \mathrm{MgCl}, 15 \mathrm{mM}$ Tris (pH 7.4), $0.3 \mathrm{M}$ $\mathrm{NaCl}$ ). After $90 \mathrm{~min}$ of centrifugation at $39.000 \mathrm{rpm}$ in an SW41-Ti rotor at $4^{\circ} \mathrm{C}$, the absorbance at $254 \mathrm{~nm}$ was measured continuously as a function of gradient depth in a BioRad Laboratories UV monitor. To assess overall translation efficiency, we integrated the area under the curve that represented mRNA attached to 2 or more ribosomes, and compared this to the total area under the curve. 


\section{RNA isolation}

Samples were collected in $0.55 \mathrm{M}$ final concentration of guanidinium- $\mathrm{HCl}$, subjected to 4 precipitation steps (ethanol, sodium acetate and lithium chloride), one phenol-chloroform extraction and one column extraction (RNeasy Mini Kit, Qiagen) with extensive washing in between. RNA quantity and quality was checked using the ND-1000 spectrometer (NanoDrop Technologies) and RNA Nano LabChip kit on the 2100 Bioanalyzer (Agilent) respectively.

\section{Affymetrix gene array measurements}

20ng RNA was pooled from 3 independent experiments, representing total and highly efficiently translated RNA from aerobic and hypoxic cells. The RNA was processed according to the manufacturer's standard protocol (Affymetrix) for 2-cycle amplification. The biotin-labelled samples were hybridized to HG U133 Plus 2.0 chips. GCOS software (Affymetrix) was used for the absolute call (present, marginal, absent) of 54.675 probe sets. Intensities were filtered with a threshold of 200 . Two lists of genes were generated by sorted according to (Polysomal Hypoxia)/(Polysomal Normoxia) intensity ratios or (Total Hypoxia)/(Total Normoxia) intensity ratios respectively.

\section{Quantitative PCR analysis}

The RNA was reverse transcribed using random decamers (Ambion) and MML reverse transcriptase (Sigma-Aldrich). Real-time PCR was performed in ABI 7500 (Applied Biosystems). The abundance of the following genes were detected with SYBR® Green I (Applied Biosystems) using the indicated forward and reverse primers: $\beta$-Actin: F-accatggatgatgatatcgcc and R-gccttgcacatgccgg; ATF-4: F-tggccaagcacttcaaacct and Rgttgttggagggactgaccaa; DDIT3: F-ggagcatcagtcccccactt and Rtgtgggattgagggtcacatc; VEGF: F-gactccggcggaagcat and Rtccgggctcggtgattta. The following genes were measured using TaqMan $\AA$ Gene Expression Assays (Applied Biosystems): 18SrRNA: Hs99999901_s1 
and EGLN3: Hs00222966 m1. The abundance of every gene was calculated using standard curves, and the relative gene abundances were normalized by 185 signal.

\section{Results}

We have reported that hypoxia results in a rapid inhibition of MRNA translation. In order to investigate the potential contribution of this inhibition on protein expression we analyzed the cellular proteome after short (1 hr) and long (24 hrs) exposures to hypoxia. The proteome from aerobic or hypoxic HeLa cells was separated in two dimensions on the basis of charge and mass and proteins visualized by staining with SYPRORuby. The early time point ( $1 \mathrm{hr}$ ) was chosen to minimize the contribution of changes in transcription on protein expression. Thus, most of the changes in protein expression are due to post-transcriptional changes including the inhibition of MRNA translation which is maximal at this time. We compared the results at $1 \mathrm{hr}$ with the proteomic changes in chronically (24 hours) hypoxic cells, where differential gene expression was assumed to be affected by both transcriptional and post-transcriptional changes. This allowed us to assess the relative importance of post-transcriptional regulation during hypoxia. This experiment was repeated in triplicate thus producing 3 separate 2D-gels for each condition (representative gels from each experimental condition are shown in figure $1 \mathrm{~A})$. Proteins were matched across all gels to identify those that consistently showed changes during each of the experiments. The levels of these matched proteins during hypoxia and normoxia are plotted in figure $1 \mathrm{~B}$. Table 1 also lists the number of protein spots that showed strong consistent expression under one condition, but were not present in the other. These spots are therefore likely to represent real induced or repressed proteins, and are not a result of technical artifacts. The substantial change in the hypoxic proteome visible within 1 hour suggests that post-transcriptional events are important regulators of protein expression under these conditions. 
A

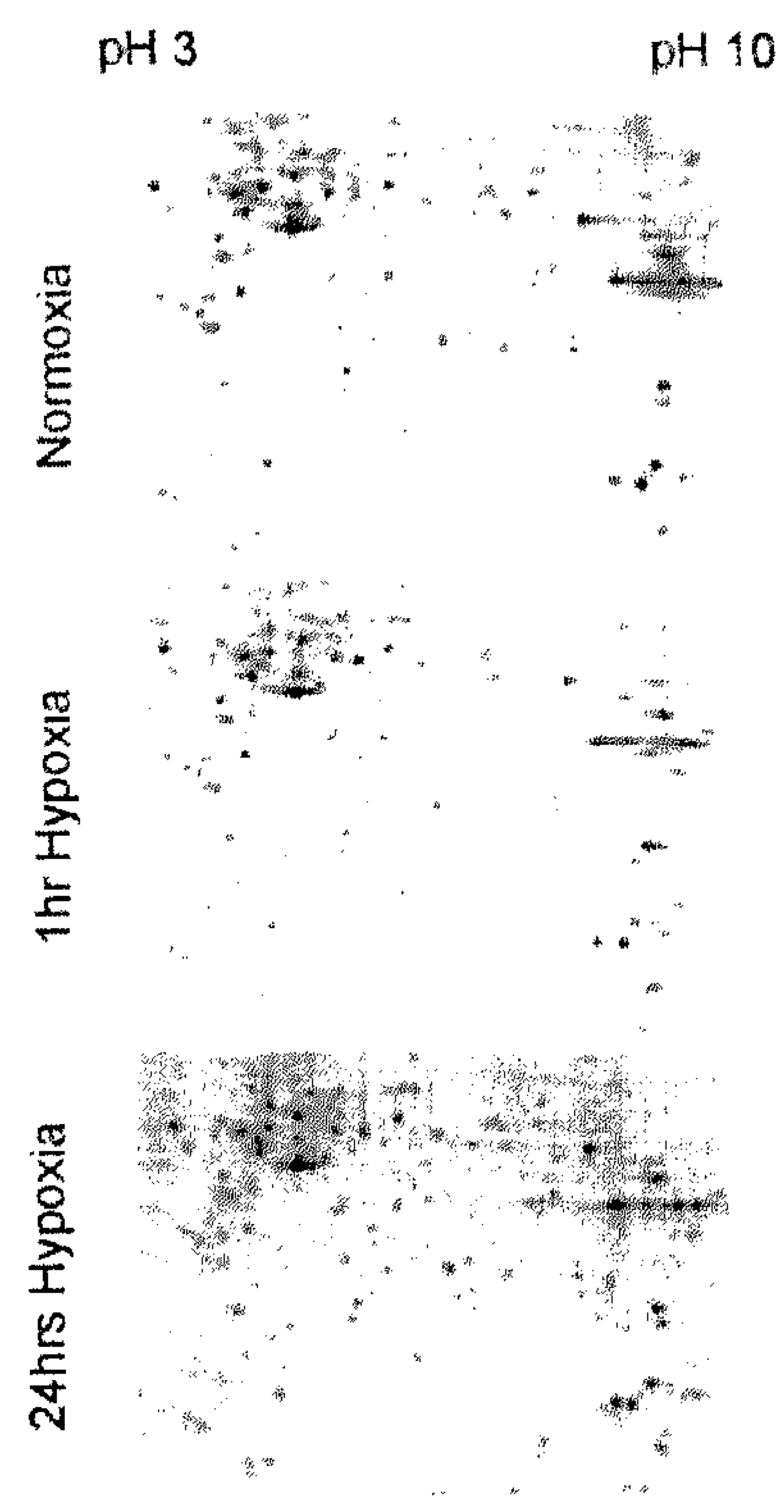

$\mathrm{B}$

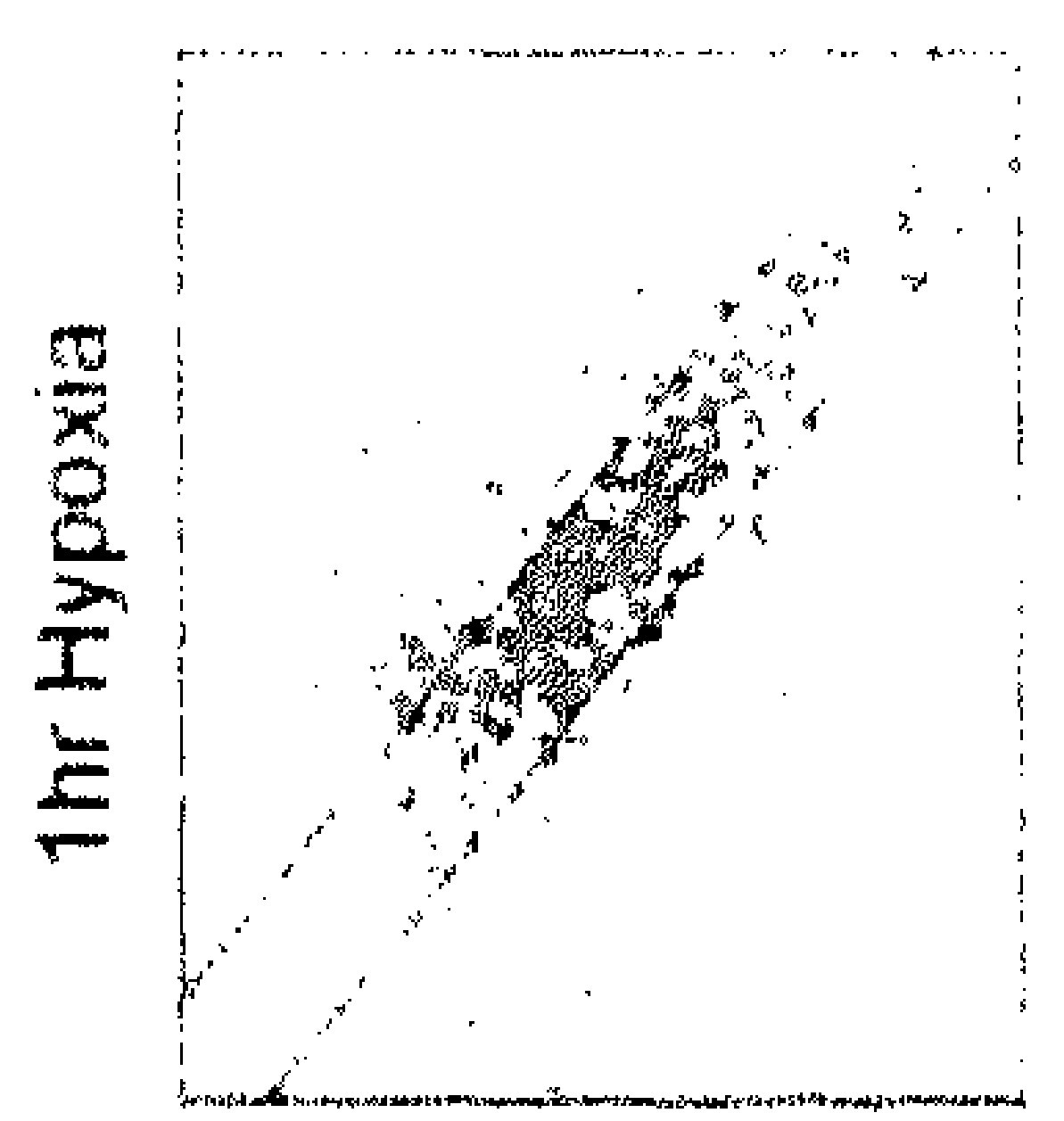

Normoxia

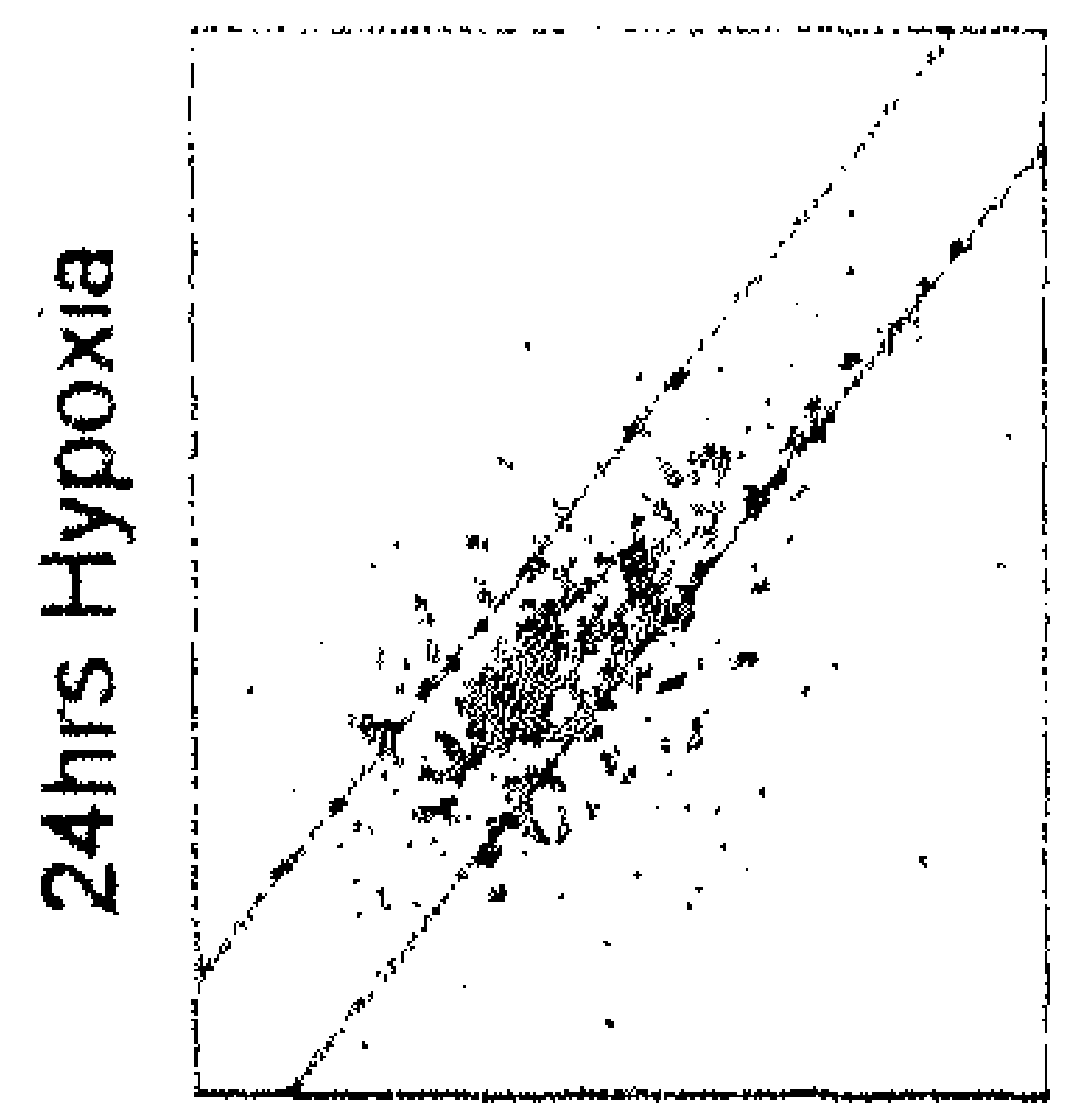

Normoxia

Figure 1. Hypoxia-induced changes in the proteome. Hela cells were exposed to hypoxia for 0,1 or 24 hours. Whole cell extracts were separated by 2D gel electrophoresis. (A): The resolved proteins were stained with SYPRO Ruby dye and imaged on the Molecular Imager FX (Bio-Rad). (B): The gels were analysed using PDQuest software (Bio-Rad). The relative abundance of each matched protein during anoxia vs. hypoxia was plotted with the diagonal lines representing 2 -fold changes. 
Table 1. Hypoxia-induced changes in the proteome.

$\begin{array}{cccccc}\begin{array}{c}\text { Hours of } \\ \text { hypoxia }\end{array} & \begin{array}{c}\text { Detected } \\ \text { proteins }\end{array} & \begin{array}{c}\text { Matched } \\ \text { proteins }\end{array} & \begin{array}{c}\text { >2-fold } \\ \text { change }\end{array} & \begin{array}{c}\text { Present } \\ \text { only in } \\ \text { normoxia }\end{array} & \begin{array}{c}\text { Present } \\ \text { only } \\ \text { in hypoxia }\end{array} \\ 0 & 1440 & & & & \\ 1 & 982 & 685 & 70 & 30 & 10 \\ 24 & 1102 & 882 & 77 & 20 & 25\end{array}$

Regulation of mRNA translation efficiency, which is known to occur during hypoxia, is a possible mechanism that can influence the rapid changes in protein expression shown above. To assess overall mRNA translation efficiency in DU145 cells during hypoxic conditions, we separated cell lysates on sucrose gradients and then measured RNA levels as a function of gradient depth. The resulting profiles are shown in figure $2 A$. The two shallowest peaks (readily detected in the aerobic sample) represent the 405 and 605 ribosomal subunits respectively. The subsequent peaks represent mRNAs with increasing numbers of attached ribosomes. Efficiently translated mRNA is associated with many ribosomes (polysomes) simultaneously and therefore sediments deep in the gradient. Figure 2A demonstrates that hypoxia inhibits overall translation in DU145 cells since there is a clear reduction in the high molecular weight polysomal RNA. The percentage of ribosomes participating in translation can be determined by integrating the polysomal area and dividing by the total integrated area. The average results from three independent experiments are presented in figure $2 \mathrm{~B}$. This figure shows that the fraction of ribosomes participating in translation dropped from $74 \%$ in aerobic cells to $45 \%$ after 4 hours of hypoxia. We also calculated the fraction participating in highly efficient translation (figure $2 \mathrm{~A}$ ), which we defined as mRNA with 5 or more ribosomes. This fraction dropped from $48 \%$ to $20 \%$ after 4 hrs of hypoxia. 
A

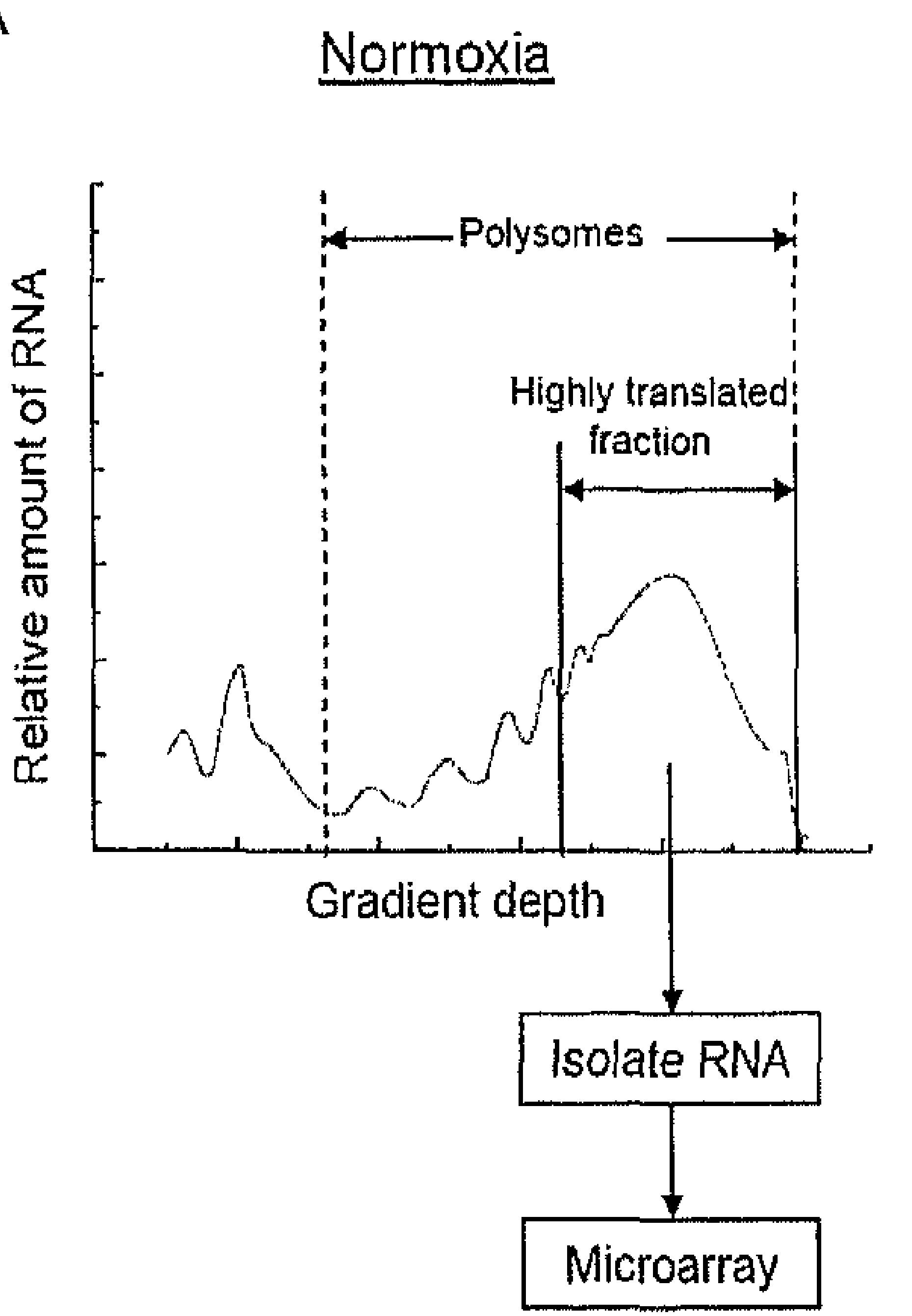

Hypoxia

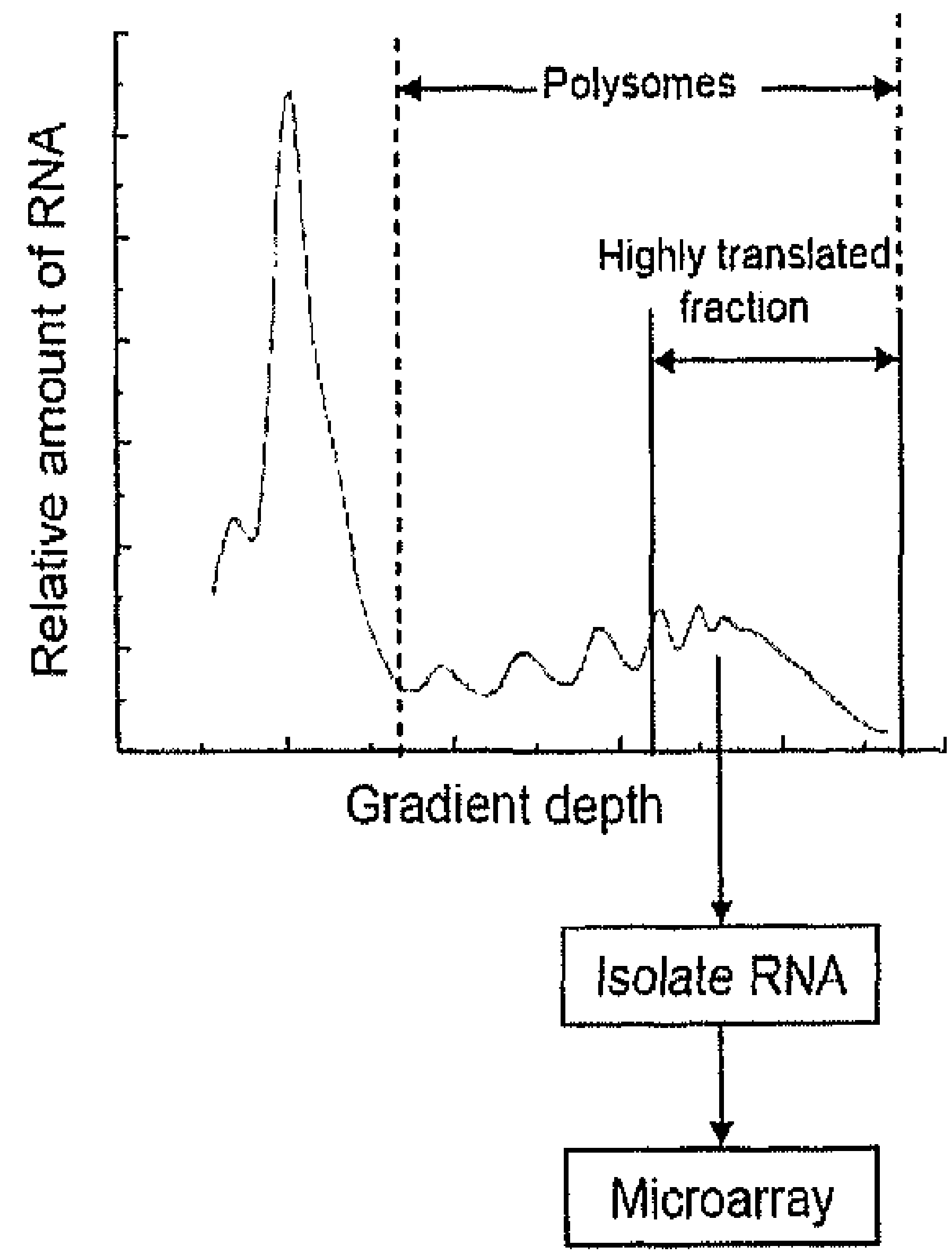

$\mathrm{B}$

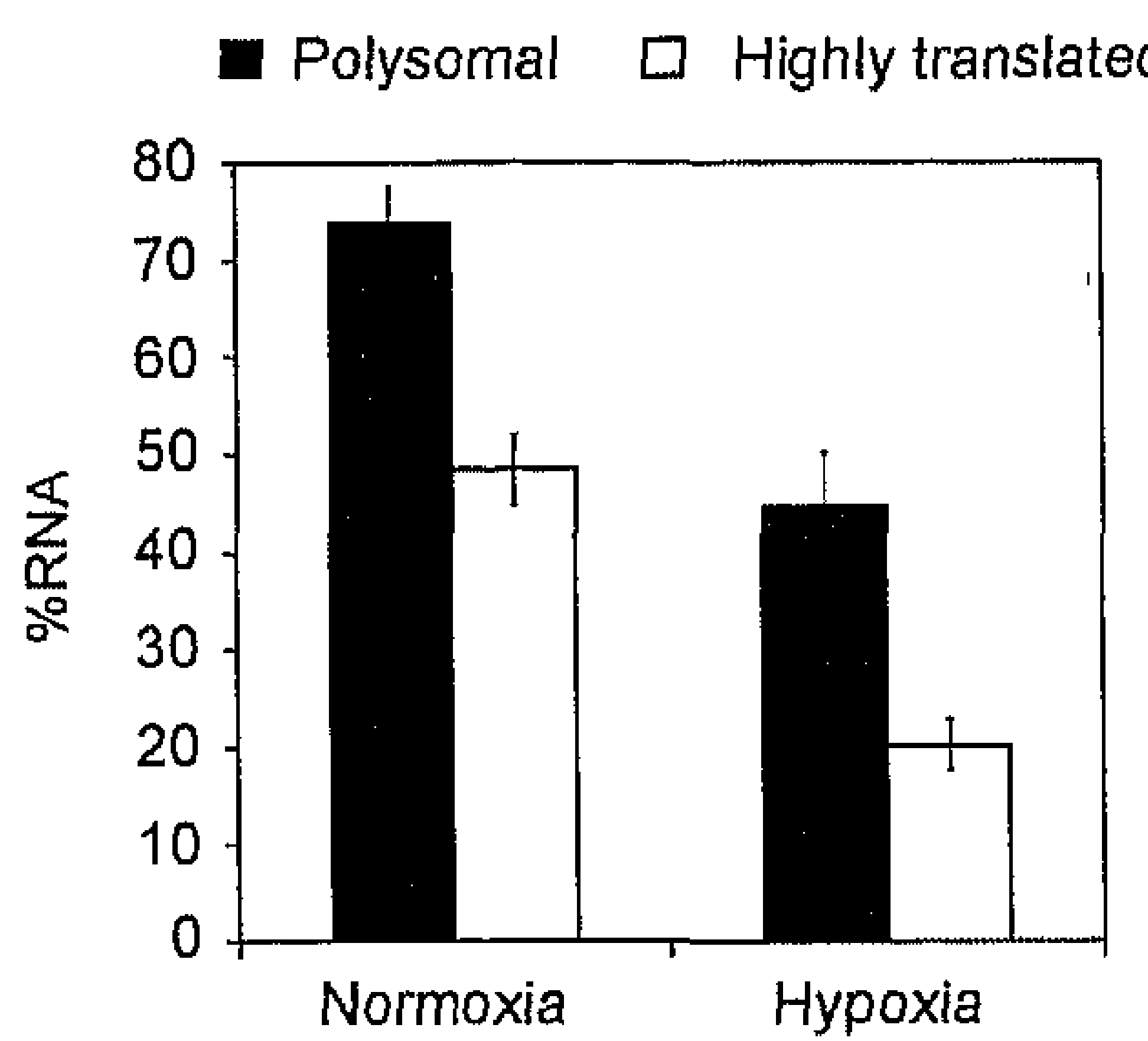

Figure 2. Hypoxia inhibits mRNA translation. DU145 cells were exposed to hypoxia for 0 or 4 hours. Whole cell extracts were separated by sedimentation through sucrose gradients. (A): Optical density (O.D.) at $254 \mathrm{~nm}$ as a function of depth in the gradient. The area representing RNA that participates in complexes containing 2 or more ribosomes is marked "polysomes". RNA was isolated for microarray and PCR analysis from the fraction indicated "highly efficient transiation". (B): Quantification of translation efficiency as the percentage of rRNA participating in complexes of two or more ribosomes. This equals the integration of the area under the "polysomes" part of the curve divided by the total area under the curve. The fraction participating in "highly efficient translation" is also indicated. 
In order to investigate the potential contribution of changes in mRNA translation on hypoxia induced gene expression, we utilized microarray technology. Changes in mRNA translation were assessed by isolating RNA from the highly efficiently translated polysomal fractions as depicted in figure 2A. Transcriptional changes were assessed by isolating total mRNA from the same samples. Since this technique allowed us to evaluate both transcriptional and translational contributions to differential gene expression, we chose to measure these entities after 4 hours of hypoxia. At this time point both transcriptional and translational control of gene expression are expected to be active, and with the microarray technique we could directly compare these events. In addition to providing more information, this also allowed us to validate our results through literature comparison, since many hypoxia-induced transcriptional changes are well described. Equivalent RNA amounts from three independent experiments from cultures growing under normoxia or following 4 hours of hypoxia were pooled and then assessed using Affymetrix microarray chips containing 54,675 probe sets (including 38,500 well-characterized human genes).

Figure 3 shows the scatter plots of gene intensities (log base 2) for hypoxic and aerobic samples. For all genes that demonstrate a less than 2-fold change during hypoxia, the plotted points fall within the two indicated parallel lines on these plots. Figure $3 \mathrm{~A}$ summarizes the transcriptional (total RNA) changes during hypoxia. Of the 22,332 gene probe sets that could be evaluated, 654 (3\%) were induced more than 2 fold during hypoxia with 76 of these increasing more than 4-fold. Changes in the highly translated RNA fraction are summarized in Figure 3B. In this highly translated fraction we found that 1082 genes $(\sim 5 \%)$ increased more than 2-fold during hypoxia with 120 of these increasing more than 4-fold. The amount of an individual gene in the highly translated mRNA fraction reflects both the total mRNA available for translation, as well as the gene's translation efficiency. However, since both the total mRNA and the highly translated fraction of mRNA are known, it is possible to 


\section{Chapter 4}

calculate the relative translation efficiency for each gene on the array. This is simply equal to the signal in the highly translated fraction divided by the signal from the total cell. We calculated the translation efficiency for each gene under both aerobic and hypoxic conditions to determine those genes exhibiting the largest increases in translation efficiency. Figure $3 \mathrm{C}$ shows that the translation efficiency of 508 genes increased more than 2-fold with 41 of these increasing more than 3-fold and 5 more than 4-fold. These results demonstrate that although overall translation is inhibited during hypoxia, a significant number of genes are induced through specific increases in their translation efficiency.

Table 2 shows a ranked list of the 50 genes showing the largest increase in mRNA translation during hypoxia (derived from figure $3 B$ ). Table 2 also lists the increase in total mRNA abundance and hence the component that can be attributed to changes in mRNA production and/or stability. The change in mRNA translation efficiency during hypoxia can be deduced by comparing these values. Of the 50 most highly induced genes, 11 demonstrated a significant contribution from changes in translation efficiency (more than 2-fold increase in translation efficiency). For each of these genes, their rank within the top 50 was much higher than their rank evaluated by the increase in total mRNA levels. As shown in Table 2, a majority of the 50 genes showing the largest changes in translation have previously been shown to be induced by hypoxia either at the mRNA or protein level. This result helps to validate the results presented here. To further validate our microarray data, we performed quantitative real-time $P C R$ ( $q P C R$ ) on several of the genes shown to be transcriptionally upregulated. The results from these validation experiments are shown in figure 4. As a negative control, we measured the abundance of $\beta$-actin, which was not induced by hypoxia on the microarray. As expected, $\beta$-actin was also not induced when measured by QPCR. We also measured the mRNA abundance for ATF-4, EGLN3, VEGF and DDIT3. The results from the GPCR were consistent with the data from the microarray. In general, the induction factors were somewhat higher when measured by GPCR. 
This might be related to the fact that the gene abundance using this method is normalized by the abundance of $18 \mathrm{~S}$ rRNA, which may fall slightly during hypoxia. Nonetheless, the GPCR results verified the data obtained by microarray analysis.

A

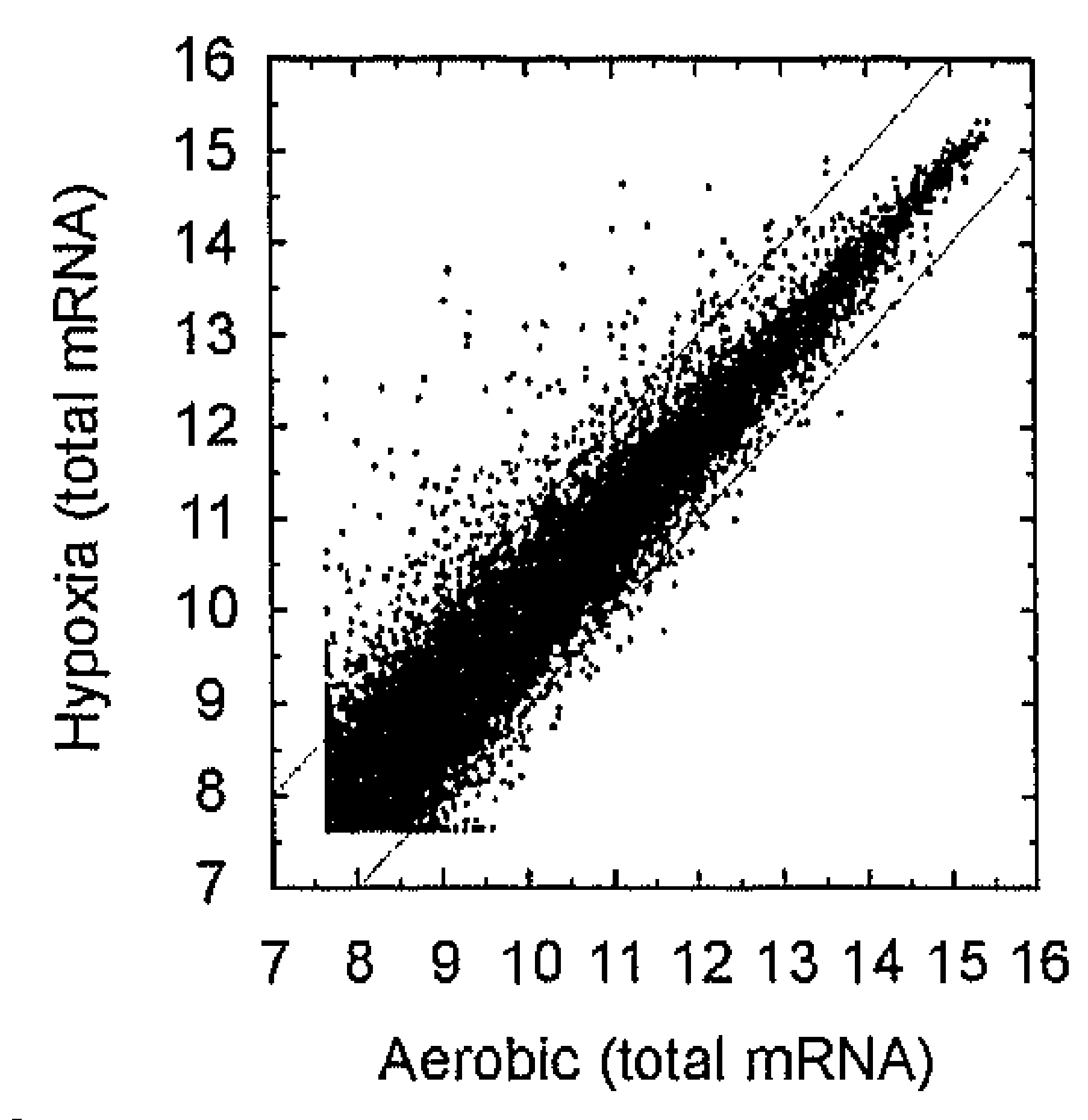

C

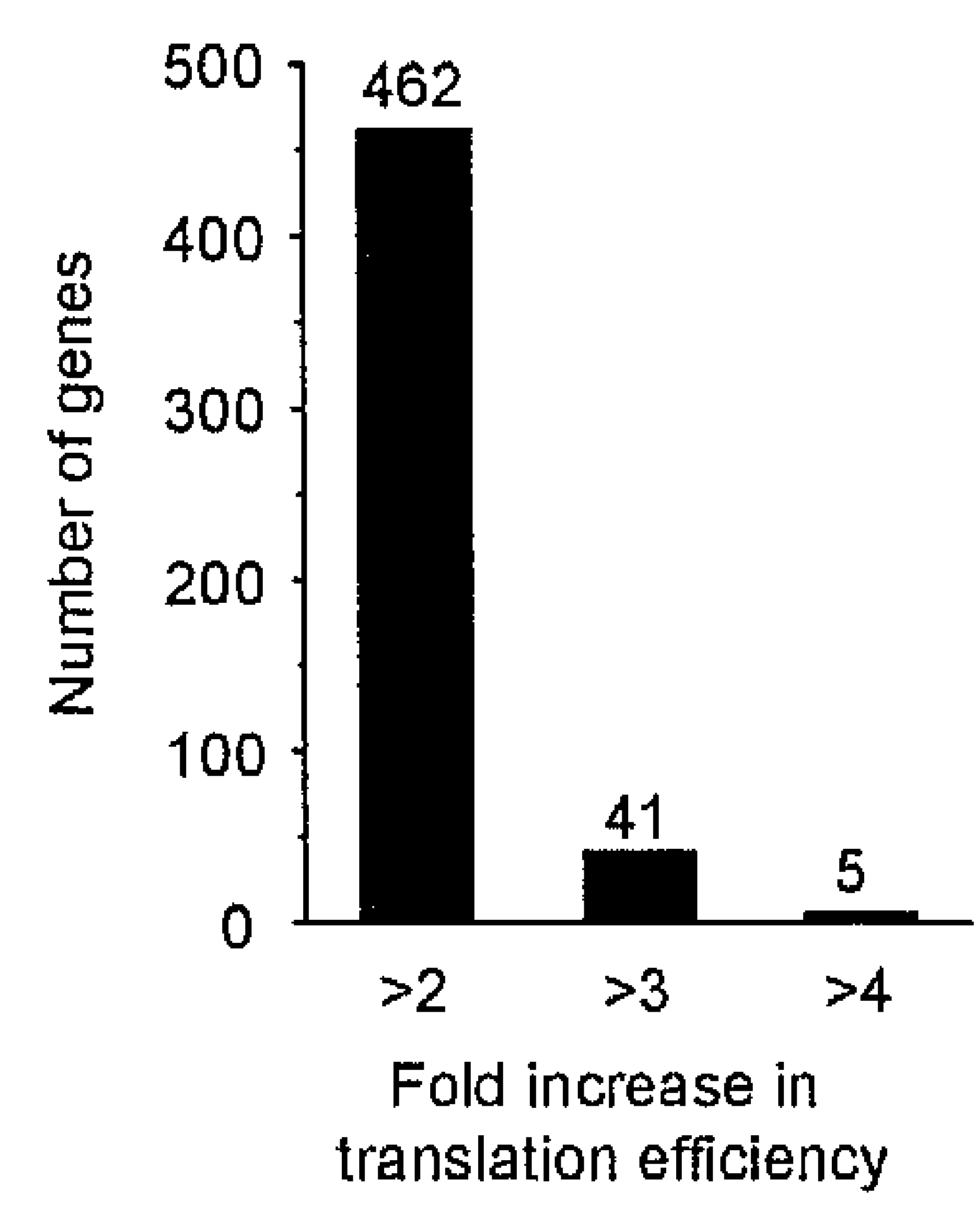

$B$

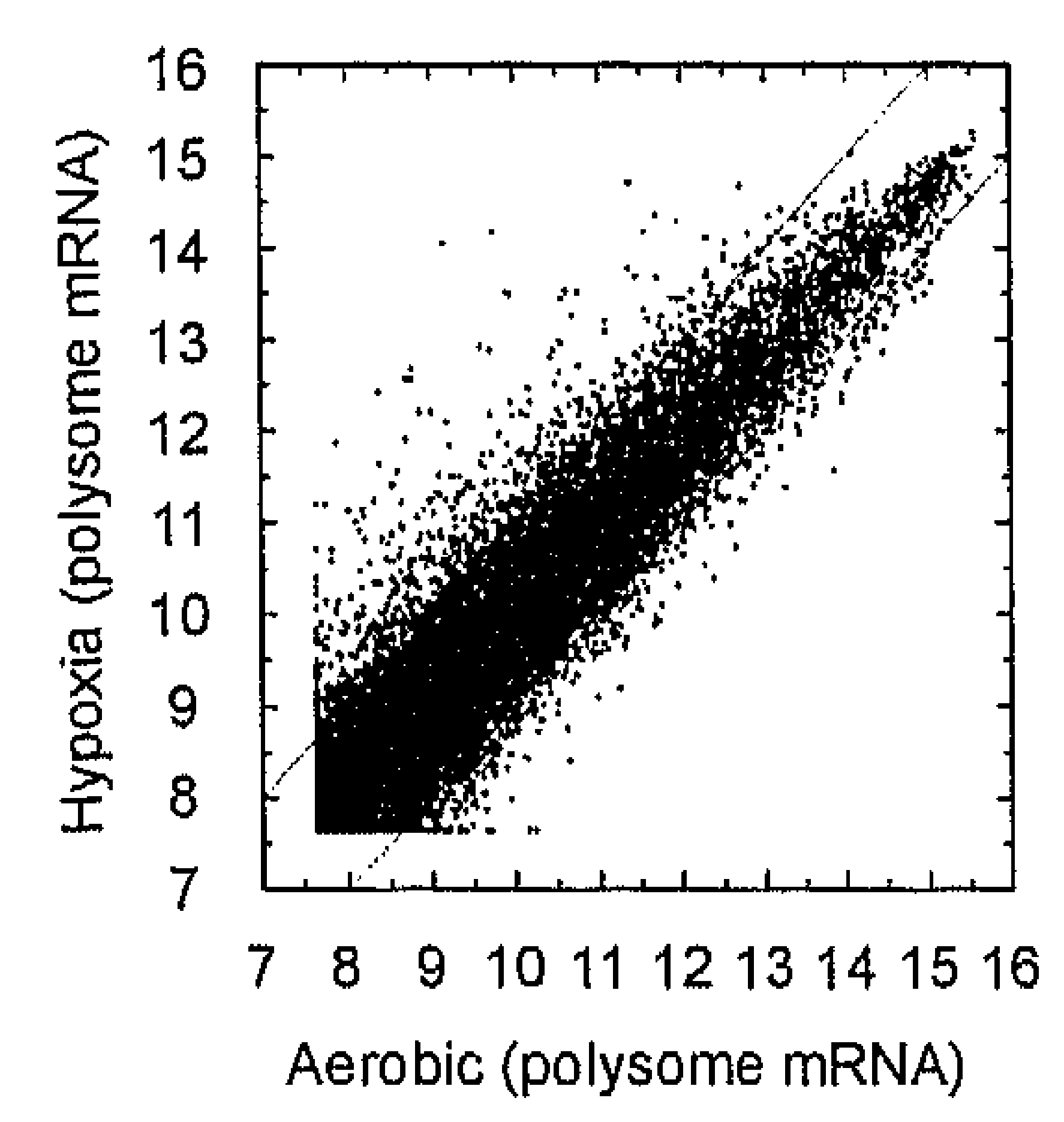

Figure 3. Microarray signal distributions. Total and polysomal RNA isolated from DU145 cells after exposure to hypoxia for 0 or 4 hours was hybridized to Affymetrix microarray chips. (A) and (B) Logarithm of raw (not normalized) intensities of probe sets under hypoxia vs. anoxia for total mRNA. Diagonal lines represent the threshold for 2-fold expression changes. (A) Total, i.e. unfractionated mRNA in aerobic versus hypoxic samples. (B) mRNA with high translation efficiency in aerobic versus hypoxic samples. (C) Representation of the number of genes with hypoxia induced translation efficiency, calculated by dividing the gene abundance from the "highly efficient translation" by that obtained from the unfractionated sample. 
Table2. Genes most highly induced by hypoxia measured by microarray.

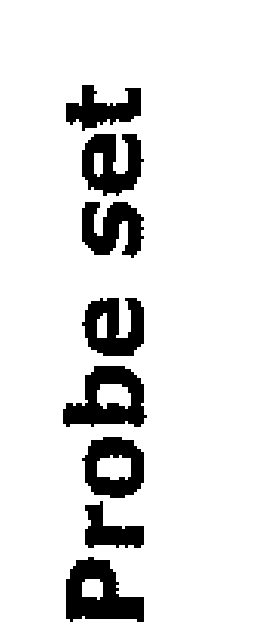

202934_at HK2 221009_s_at

202769_at

209357_at

217028_at

235857_at

218507_at

204284_at

229830_at

203438_at

227337_at

242517_at

223333_s_at

202887_s_at

202364_at

209383_at

228499_at

206424_at

209201_x_at

218274_s_at

212366_at

221567_at

218149_s_at

201170_s_at

201313_at

208763_s_at

203574_at

201466_s_at JUN

205463_s_at PDGFA

202843_at

CXCR4

HIG2

PDGFA

STC2

Lrp2bp

DDIT

MXI

DDIT3

PFKFB

CXCR4

DNAJB

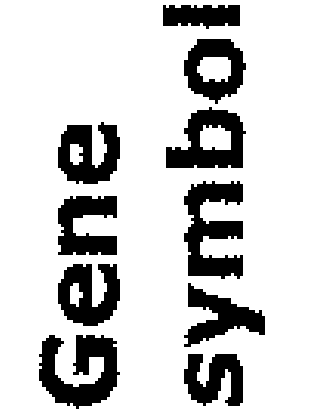

ANGPTL4

CCNG2

CITED2

KCTD11

PPP1R3C

GPR54

ANGPTL4 13

CYP26A1

FLI10415 20

$\begin{array}{lllll}Z N F 292 & 21 & 52 & 8.3 & 4.7\end{array}$

$\begin{array}{llllll}\text { NOL3 } & 22 & 22 & 7.8 & 8.1 & 1.0\end{array}$

$\begin{array}{llllll}Z N F 395 & 23 & 62 & 7.8 & 4.3 & 1.8\end{array}$

$\begin{array}{llllll}\text { BHLHB2 } & 24 & 26 & 7.5 & 7.2 & 1.0\end{array}$

ENO2 25

DSIPI 26

NFIL3

28

$2959 \quad 7.3$

$0.8 \quad 10.1 \quad 1.1$

$10.2 \quad 17.2 \quad 0.6$

$\begin{array}{lll}0.2 & 13.2 & 0.8\end{array}$

1.50 .9

9

20.30 .5

$\begin{array}{lll}9.2 & 22.2 & 0.4\end{array}$

$6.5 \quad 1.4$

$8.1 \quad 1.1$

7

$4.1 \quad 1.8$

$\begin{array}{llll}29 & 7.5 & 6.7 & 1.1\end{array}$

.56

$0 \quad 1$

.34 .4
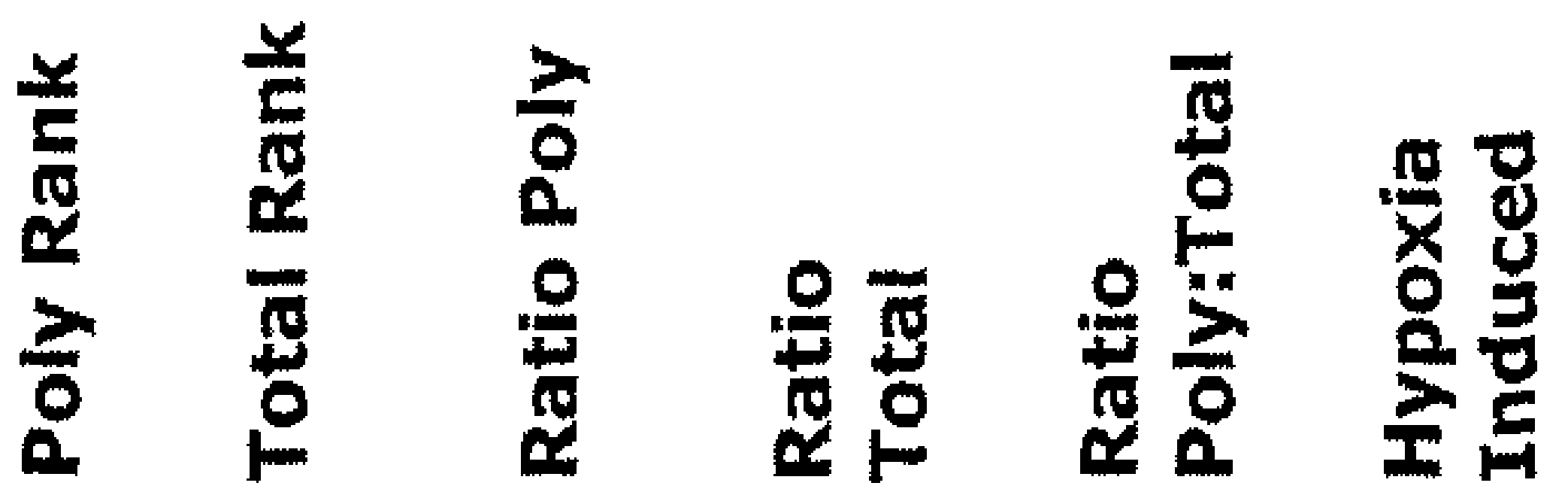

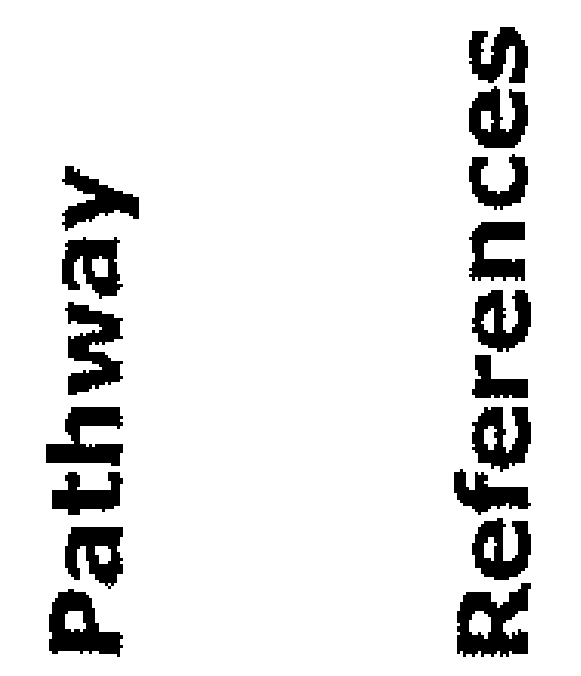

$\mathrm{H}^{1} \quad$ [26, 69]

H [3,39]

$H \quad\{40,71\}$

H $\quad 5,60]$

H $\quad[61,69]$

H [44.69]

$\mathrm{H} \quad[40,60]$

[19]

$H, U^{2} \quad[25,40]$

yes

$\mathrm{H} \quad$ [3,39]

yes

H $\quad[9,40]$

yes

H [69]

$\mathrm{H}, \mathrm{U} \quad[12,42]$

H $\quad[48,60]$

yes $\quad H \quad[61,69]$

0
8

yes

$H \quad[71,72]$

yes

$[60]$

$\begin{array}{llll}24 & 7.5 & 7.7 & 1.0\end{array}$

.2 yes

$H, \cup \quad[36,60,64]$

$4.4 \quad 1.7$ yes

[19]

U [35] 
Continued Table2. Genes most highly induced by hypoxia measured by microarray.

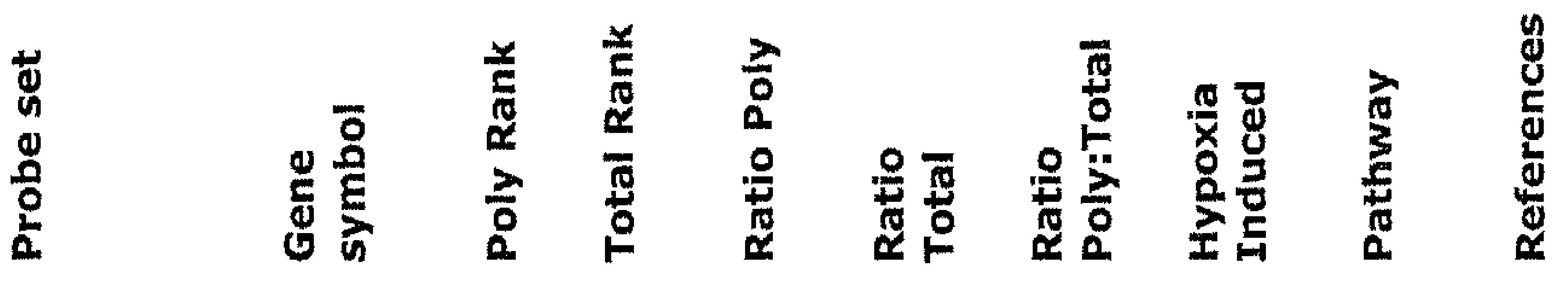

\begin{tabular}{|c|c|c|c|c|c|c|c|c|c|}
\hline 226863_at & & 31 & 123 & 7.3 & 3.4 & 2.2 & & & \\
\hline 231798_at & NOG & 32 & 23 & 7.0 & 7.8 & 0.9 & & & \\
\hline 1554452_a_at & HIG2 & 33 & 19 & 6.8 & 8.3 & 0.8 & yes & $\mathrm{H}$ & {$[14,69]$} \\
\hline 203439_s_at & STC2 & 34 & 18 & 6.8 & 8.6 & 0.8 & yes & $\mathrm{H}, \mathrm{U}$ & {$[15,40]$} \\
\hline 215446_s_at & LOX & 35 & 114 & 6.8 & 3.4 & 2.0 & yes & $\mathrm{H}$ & {$[60,69]$} \\
\hline 212368_at & ZNF292 & 36 & 37 & 6.7 & 5.8 & 1.2 & & & \\
\hline 202973_x_at & FAM13A1 & 37 & 68 & 6.7 & 4.1 & 1.6 & & & \\
\hline 200632_s_at & NDRG1 & 38 & 17 & 6.4 & 8.9 & 0.7 & yes & $\mathrm{H}$ & {$[24,60]$} \\
\hline 241348_at & Fப10997 & 39 & 130 & 6.3 & 3.3 & 1.9 & & & \\
\hline 1554462_a_at & DNAJB9 & 40 & 35 & 6.2 & 6.1 & 1.0 & & U & [35] \\
\hline 210512_s_at & VEGF & 41 & 39 & 6.1 & 5.6 & 1.1 & yes & $H, U$ & $\begin{array}{l}{[24,26,43,} \\
68]\end{array}$ \\
\hline 204285_s_at & PMAIP1 & 42 & 159 & 6.1 & 3.0 & 2.0 & yes & $\mathrm{H}$ & [31] \\
\hline 209339_at & SIAH2 & 43 & 459 & 6.0 & 2.2 & 2.7 & yes & & {$[50]$} \\
\hline 211527_x_at & VEGF & 44 & 270 & 5.9 & 2.5 & 2.3 & yes & $\mathrm{H}, \mathrm{U}$ & $\begin{array}{l}{[24,26,43,} \\
68]\end{array}$ \\
\hline 224610_at & RNU22 & 45 & 286 & 5.9 & 2.5 & 2.4 & & & \\
\hline 202672_s_at & ATF3 & 46 & 11 & 5.6 & 12.0 & 0.5 & & $\mathrm{U}$ & [28] \\
\hline 224797_at & ARRDC3 & 47 & 56 & 5.5 & 4.6 & 1.2 & & & \\
\hline 224741_x_at & GAS5 & 48 & 277 & 5.5 & 2.5 & 2.2 & yes & & [29] \\
\hline 219232_s_at & EGLN3 & 49 & 433 & 5.4 & 2.2 & 2.4 & yes & $\mathrm{H}$ & {$[2,44]$} \\
\hline 204286_s_at & PMAIP1 & 50 & 174 & 5.4 & 2.9 & 1.9 & yes & $H$ & [31] \\
\hline
\end{tabular}




\section{Discussion}

There is substantial evidence that tumor oxygenation varies dynamically in time at frequencies that would be difficult to compensate for by changes in transcription. Thus, it is not clear to what degree the cellular proteome, and ultimately cell behavior, changes during acute exposures to hypoxia. To address this question we assessed the effect of both acute ( 1 hour) and chronic (24 hours) hypoxia on the overall pool of expressed proteins. Both 1 hour and 24 hours of hypoxia clearly caused significant changes in the proteome, as demonstrated by a large number of proteins showing a greater than 2-fold change in expression. As listed in Table 1, significantly fewer protein spots were detected during hypoxia compared to normoxia, consistent with hypoxia-induced inhibition of mRNA translation (and transcription at later time points). Of the proteins that were detected in all conditions, approximately $10 \%$ changed their expression 2-fold or more during hypoxia. Of these, $59 \%$ had increased expression during 1 hour hypoxia, versus 34\% after 24 hours. The results shown in figure 1 and table 1 demonstrate that significant changes in the proteome take place very early in the hypoxic exposure, at a time where transcriptional changes are thought to contribute to a negligible extent.

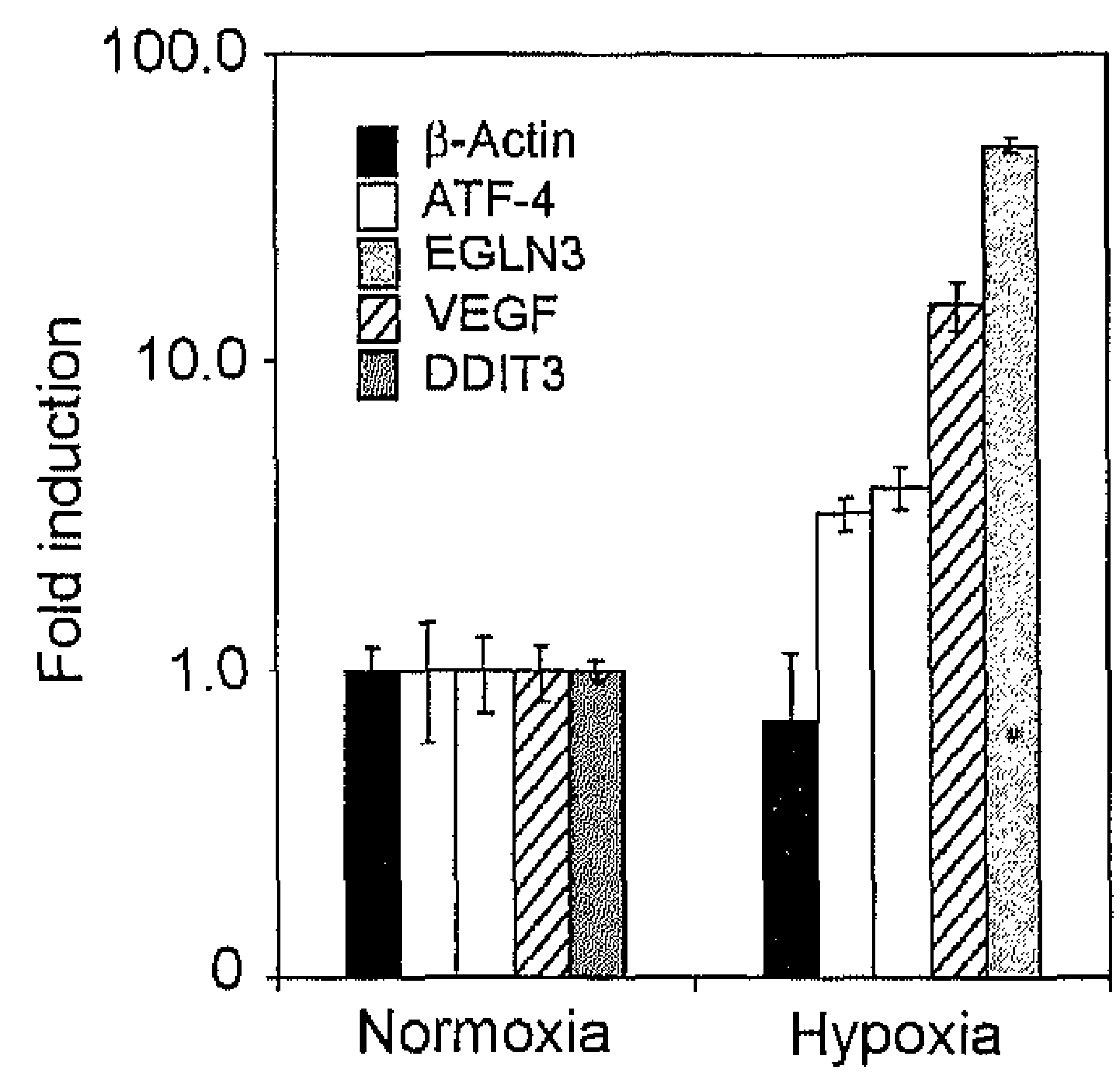

Figure 4. Microarray validation by quantitative PCR. DU145 cells were exposed to hypoxia for 0 or 4 hours. Gene-specific mRNA abundance was measured for $\beta$-actin, ATF-4, EGLN3, VEGF and DDIT3 using quantitative real-time PCR. Gene abundance was normalized by $18 \mathrm{~S}$ rRNA signal and the induction factors were calculated using the abundance measured in three independent experiments. The standard error of the mean is shown. 
These data provide evidence that a non-transcriptional mechanism is important for the changes in protein expression that occur during hypoxia. Although we argue that gene-specific mRNA translation can contribute significantly to the observed changes, other mechanisms may also be important contributors, including both mRNA and protein stabilization.

A strong suppression of mRNA translation was observed after 4 hours of hypoxia. However, the global decrease in translation efficiency assayed in this way represents the average reduction in translational efficiency for all genes. The change in translational efficiency for any particular gene under these conditions may differ significantly from this global average. This variability is thus a potentially important contribution to changes in protein expression during hypoxia. In order to evaluate this contribution we used microarrays to characterize both transcriptional and translational changes of individual genes. After 4 hours of hypoxia we found a substantially higher number of genes that showed significant increases in translation in comparison to the number showing only transcriptional changes. This is due to the fact that increased translation arises from increases in both mRNA abundance and in gene-specific translation efficiency. Importantly, these translated genes accurately reflect de novo protein production and thus are likely contributors to biological behavior. The gene found to show the largest increase in translation during hypoxia was the HIF-1 target gene hexokinase $2(\mathrm{HK}-2)$, which is involved in glucose metabolism ${ }^{38}$. The protein production of HK-2 increased by 30fold during 4 hours of hypoxia versus normoxia, while the mRNA abundance in the cells increased by 25 -fold. The interpretation of this result is that $\mathrm{HK}-2$ is both transcriptionally and translationally induced, with the transcriptional component being dominant.

A majority of the genes showing the highest increases in translation have previously been shown to be targets of the HIF transcription factors (table 2). The criteria for this annotation were non stringent and genes that contain putative HREs in their promoter, or that are induced by either overexpression of HIF or loss of pVHL have been included together with 
bona fide HIF targets. It is clear from the table that the potential influence of HIF through hypoxia-induced transcription is significant. However, the HIF target genes displayed a large spread in translation efficiency during hypoxia, ranging from 0.4 for PFKFB4 to 3.0 for CITED-2. CITED-2 was highly induced during hypoxia (number 4 on the list) with a 16-fold increase in translation and only a 5-fold increase in total mRNA. This gene product binds $\mathrm{p} 300$ and thereby inhibits the HIF-1-p300 interaction required for HIF-1-dependent gene transcription 39,40 . Hypoxia has previously been shown to induce both MRNA and protein levels of CITED-2 ${ }^{40}$, and the results presented here indicate that gene-specific up regulation of mRNA translation contributes significantly to the increased protein levels. Interestingly, the CITED-2 promoter also contains three $\mathrm{HREs}^{40}$, indicating that it is a part of a negative feedback loop controlling expression of HIF-1 target genes. Apparently, CITED-2 mRNA is in addition able to bypass the overall repression of mRNA translation to ensure its robust expression during hypoxia.

Other genes that have a high translational component of induction include hypoxia-inducible protein 2 (HIG-2), MAX interactor 1 (MXI-1), zinc finger protein (ZNF) 292 and 395 and enolase 2 (ENO-2). As exemplified by HK-2 and CITED-2, several of the genes shown in table 2 are induced both at the transcriptional level and through increased mRNA translation efficiency. It is conceptually not surprising that transcriptionally activated mRNA species possess the ability to be preferentially translated. Without this ability, the transcriptional induction would clearly be inefficient and result in uneconomical use of the cells limited energy sources.

The mechanistic basis for preferential translation is not well understood, although elements in the regulatory (i.e. untranslated) parts of the mRNA seem to be important. ATF-4 is an example of a gene that, paradoxically, is more efficiently translated during ER stress when global translation is inhibited through PERK-dependent eIF2 $\alpha$ phosphorylation ${ }^{35}$. PERK is also activated during hypoxia and necessary for at least a 
component of the hypoxia-induced inhibition in translation ${ }^{31}$. ATF-4 is thought to be translationally induced during eIF2a phosphorylation because its upstream open reading frames (UORFs) in the $5^{\prime}$ untranslated region ( $5^{\prime} U T R$ ) of the mRNA prevent proper ribosome initiation under normal conditions ${ }^{35}$. Phosphorylation of eIF $2 \alpha$ makes initiation less efficient which increases the probability that ribosomes will read through the false UORFs and start translating at the bona fide start site. In our microarray, ATF-4 was indeed translationally induced during hypoxia, consistent with results from a previous microarray experiment performed after a longer hypoxia exposure in HeLa cells ${ }^{41}$. Here, we obtained an induction of 3.5 in the polysomes versus 2.3 in the totals, resulting in a poly/total ratio of 1.52 . It is also noteworthy that 8 of the 50 top induced genes listed in table 2 have been previously implicated in the UPR which requires ATF4 activation.

Gene-specific translation during hypoxia has also been reported for mouse HIF-1 $1 \alpha$ and human VEGF. These genes have been reported to contain functional internal ribosomal entry sites (IRES) in their $5^{\prime} U_{T R}{ }^{42-45}$. The presence of an IRES makes it possible for ribosomes to bind the mRNA independently of the $5^{\prime}$ cap-structure, and therefore bypasses the requirement for certain translation factors. However, although it has been shown that the IRES-dependent translation is relatively less affected by the overall hypoxia-induced translation inhibition, it is not clear to which extent it contributes to overall protein expression and phenotype. HIF-1 $\alpha$ did not appear as a translationally regulated gene on our list, perhaps indicating differences between mouse and human 5'UTRs in this gene. In contrast, VEGF was hypoxia-induced on our array and showed a poly/total ratio of 1.1 and 2.3 for two different probe sets. Many other genes had a higher contribution from changes in MRNA translation efficiency to their induction than ATF-4 and VEGF, and the molecular mechanisms responsible for this are yet to be identified.

An intriguing group of genes are those that have a large transcriptional induction, but not a corresponding increase in translation. (e.g. cyclin G2, 
GPR54, DDIT3, PFKFB4, ATF3). It is possible that this reflects different induction kinetics and that these genes will be efficiently translated after longer exposures to hypoxia. Our previous results have indicated that at least two distinct molecular pathways contribute to the inhibition of overall mRNA translation during acute and chronic hypoxia ${ }^{31,46}$. It is likely that a switch of molecular mechanisms can facilitate different groups of genes to be efficiently translated at different times. Another possibility is that the transcriptionally induced but translationally inhibited mRNAs produce proteins that are important in coping with the stress represented by reoxygenation. It may be important for the cells to have mRNA available to be translated as soon as oxygen is reintroduced and overall translation inhibition is reversed. It will thus be of interest to extend these studies of translation efficiency to cells exposed to hypoxia plus reoxygenation. Furthermore, our current results are limited to a single time point and a single oxygen concentration. It will be of interest to further evaluate the contribution of mRNA translation at a range of oxygen concentrations typical of those seen in tumors (i.e. $0.02 \%-5 \% \mathrm{O}_{2}$ ), and at different time points. The substantial change in the proteome observed as early as 1 hour after hypoxia suggests that translation control may also be important in these short exposures that may arise in vivo through transient changes in oxygenation.

In conclusion, we identified a large number of genes that demonstrate a selective ability to be translated during hypoxia exposure. Gene-specific regulation of translation therefore plays an important role in contributing to the hypoxic proteome.

\section{Acknowledgements}

This work was financially supported by Siemens, Netherlands Organization for Scientific Research (NWO) and the Dutch Cancer Society (KWF Kankerbestrijding). We thank Mieke Duysinx for technical assistance. 


\section{References}

1. Knocke $\mathrm{TH}$, Weitmann HD, Feldmann HJ, Selzer E, Potter R: Intratumoral pO2-measurements as predictive assay in the treatment of carcinoma of the uterine cervix, Radiother Oncol 1999, 53:99-104.

2. Hockel M, Schlenger K, Aral B, Mitze M, Schaffer U, Vaupel P: Association between tumor hypoxia and malignant progression in advanced cancer of the uterine cervix, Cancer Res 1996, 56:4509-4515.

3. Nordsmark $M$, Overgaard $M$, Overgaard $]$ : Pretreatment oxygenation predicts radiation response in advanced squamous cell carcinoma of the head and neck, Radiother Oncol 1996, 41:31-39.

4. Brizel DM, Sibley GS, Prosnitz LR, Scher RL, Dewhirst MW: Tumor hypoxia adversely affects the prognosis of carcinoma of the head and neck, Int J Radiat Oncol Biol Phys 1997, 38:285-289.

5. Lartigau $E$, Randrianarivelo $H$, Avril MF, Margulis A, Spatz A, Eschwege F, Guichard $M$ : Intratumoral oxygen tension in metastatic melanoma, Melanoma Res 1997, 7:400-406.

6. Rofstad EK, Maseide K: Radiobiological and immunohistochemical assessment of hypoxia in human melanoma xenografts: acute and chronic hypoxia in individual tumours, Int J Radiat Biol 1999, 75:1377-1393.

7. Vaupel $P$, Schlenger $K$, Knoop $C$, Hockel $M$ : Oxygenation of human tumors: evaluation of tissue oxygen distribution in breast cancers by computerized $\mathrm{O} 2$ tension measurements, Cancer Res 1991, 51:3316-3322.

8. Runkel S, Wischnik A, Teubner J, Kaven E, Gaa J, Melchert F: Oxygenation of mammary tumors as evaluated by ultrasound-guided computerized-pO2histography, Adv Exp Med Biol 1994, 345:451-458

9. Movsas B, Chapman JD, Horwitz EM, Pinover WH, Greenberg RE, Hanion AL, Iyer $R$, Hanks GE: Hypoxic regions exist in human prostate carcinoma, Urology 1999, 53:11-18.

10. Brizel DM, Dodge RK, Clough RW, Dewhirst MW: Oxygenation of head and neck cancer: changes during radiotherapy and impact on treatment outcome, Radiother Oncol 1999, 53:113-117

11. Fyles AW, Milosevic M, Wong R, Kavanagh MC, Pintilie M, Sun A, Chapman W, Levin W, Manchul L, Keane TJ, Hill RP: Oxygenation predicts radiation response and survival in patients with cervix cancer, Radiother Oncol 1998, 48:149-156.

12. Graeber TG, Osmanian C, Jacks T, Housman DE, Koch CJ, Lowe SW, Giaccia AJ: Hypoxia-mediated selection of cells with diminished apoptotic potential in solid tumours, Nature 1996, 379:88-91.

13. Erler JT, Cawthorne $\mathrm{CJ}$, Williams $\mathrm{KJ}$, Koritzinsky M, Wouters BG, Wilson $\mathrm{C}$, Miller C, Demonacos C, Stratford IJ, Dive C: Hypoxia-mediated downregulation of Bid and Bax in tumors occurs via hypoxia-inducible factor 1dependent and -independent mechanisms and contributes to drug resistance, Mol Cell Biol 2004, 24:2875-2889

14. Semenza GL: Regulation of hypoxia-induced angiogenesis: a chaperone escorts VEGF to the dance, J Clin Invest 2001, 108:39-40

15. Rofstad EK, Danielsen T: Hypoxia-induced metastasis of human melanoma cells: involvement of vascular endothelial growth factor-mediated angiogenesis, $\mathrm{Br}$ J Cancer 1999, 80:1697-1707

16. Veikkola $T$, Karkkainen $M$, Claesson-Welsh $L$, Alitalo $K$ : Regulation of angiogenesis via vascular endothelial growth factor receptors, Cancer Res $2000,60: 203-212$ 
17. Walton HL, Corjay MH, Mohamed SN, Mousa SA, Santomenna LD, Reilly TM: Hypoxia induces differential expression of the integrin receptors alpha(vbeta3) and alpha(vbeta5) in cultured human endothelial cells, J Cell Biochem 2000, 78:674-680

18. Young SD, Marshall RS, Hill RP: Hypoxia induces DNA overreplication and enhances metastatic potential of murine tumor cells, Proc Natl Acad Sci U S A 1988, 85:9533-9537.

19. De Jaeger K, Kavanagh MC, Hill RP: Relationship of hypoxia to metastatic ability in rodent tumours, $\mathrm{Br}$ J Cancer 2001, 84:1280-1285.

20. Plasswilm L, Tannapfel A, Cordes N, Demir R, Hoper K, Bauer J, Hoper J: Hypoxia-induced tumour cell migration in an in vivo chicken model, Pathobiology 2000, 68:99-105.

21. Semenza GL: Targeting HIF-1 for cancer therapy, Nat Rev Cancer 2003, $3: 721-732$

22. Iyer NV, Kotch LE, Agani F, Leung SW, Laughner $E$, Wenger RH, Gassmann $M$, Gearhart JD, Lawler AM, Yu AY, Semenza GL: Cellular and developmental control of $\mathrm{O} 2$ homeostasis by hypoxia- inducible factor 1 alpha, Genes Dev 1998, 12:149-162.

23. Forsythe JA, Jiang BH, Iyer NV, Agani F, Leung SW, Koos RD, Semenza GL: Activation of vascular endothelial growth factor gene transcription by hypoxiainducible factor 1, Mol Cell Biol 1996, 16:4604-4613

24. Minchenko A, Salceda S, Bauer T, Caro J: Hypoxia regulatory elements of the human vascular endothelial growth factor gene, Cell Mol Biol Res 1994, 40:35-39

25. Semenza GL, Wang GL: A nuclear factor induced by hypoxia via de novo protein synthesis binds to the human erythropoietin gene enhancer at a site required for transcriptional activation, Mol Cell Biol 1992, 12:5447-5454.

26. Madan A, Curtin PT: A 24-base-pair sequence $3^{\prime}$ to the human erythropoietin gene contains a hypoxia-responsive transcriptional enhancer, Proc Natl Acad Sci U S A 1993, 90:3928-3932.

27. Maxwell PH, Wiesener MS, Chang GW, Clifford SC, Vaux EC, Cockman ME, Wykoff CC, Pugh CW, Maher ER, Ratcliffe PJ: The tumour suppressor protein VHL targets hypoxia-inducible factors for oxygen-dependent proteolysis, Nature 1999, 399:271-275.

28. Cardenas-Navia LI, Braun R, Lewis $K$, Dewhirst M: Comparison of fluctuations of oxygen tension in FSA, $9 \mathrm{~L}$, and R3230AC tumors in rats, Adv Exp Med Biol 2003, 510:7-12

29. Cardenas-Navia LI, YU D, Braun RD, Brizel DM, Secomb TW, Dewhirst MW: Tumor-dependent kinetics of partial pressure of oxygen fluctuations during air and oxygen breathing, Cancer Res 2004, 64:6010-6017

30. Bennewith $\mathrm{KL}$, Durand RE: Quantifying transient hypoxia in human tumor xenografts by flow cytometry, Cancer Res 2004, 64:6183-6189

31. Koumenis C, Naczki C, Koritzinsky M, Rastani S, Diehl A, Sonenberg $N$, Koromilas A, Wouters BG: Regulation of Protein Synthesis by Hypoxia via Activation of the Endoplasmic Reticulum Kinase PERK and Phosphorylation of the Translation Initiation Factor eIF2alpha, Mol Cell Biol 2002, 22:7405-7416

32. Kraggerud SM, Sandvik JA, Pettersen EO: Regulation of protein synthesis in human cells exposed to extreme hypoxia, Anticancer Res 1995, 15:683-686.

33. Hochachka PW, Buck LT, Doll CJ, Land SC: Unifying theory of hypoxia tolerance: molecular/metabolic defense and rescue mechanisms for surviving oxygen lack, Proc Natl Acad Sci U S A 1996, 93:9493-9498

34. Sutherland R, Freyer J, Mueller-Klieser W, Wilson R, Heacock C, Sciandra J, Sordat B: Cellular growth and metabolic adaptations to nutrient stress 
environments in tumor microregions, Int J Radiat Oncol Biol Phys 1986, 12:611-615

35. Harding HP, Novoa I, Zhang $Y$, Zeng H, Wek R, Schapira M, Ron D: Regulated translation initiation controls stress-induced gene expression in mammalian cells, Mol Cell 2000, 6:1099-1108

36. Johannes $G$, Carter MS, Eisen MB, Brown PO, Sarnow P: Identification of eukaryotic mRNAs that are translated at reduced cap binding complex eIF4F concentrations using a cDNA microarray, Proc Natl Acad Sci U S A 1999, 96:13118-13123

37. Schroder M, Kaufman RJ: ER stress and the unfolded protein response, Mutat Res 2005, 569:29-63

38. Iyer NV, Kotch LE, Agani F, Leung SW, Laughner E, Wenger RH, Gassmann M, Gearhart JD, Lawler AM, Yu AY, Semenza GL: Cellular and developmental control of $\mathrm{O} 2$ homeostasis by hypoxia-inducible factor 1 alpha, Genes Dev $1998,12: 149-162$

39. Freedman SJ, Sun $Z Y$, Kung AL, France DS, Wagner G, Eck MJ: Structural basis for negative regulation of hypoxia-inducible factor-1alpha by CITED2, Nat Struct Biol 2003, 10:504-512

40. Bhattacharya S, Michels $C L$, Leung $M K$, Arany ZP, Kung AL, Livingston DM: Functional role of p35srj, a novel p300/CBP binding protein, during transactivation by HIF-1, Genes Dev 1999, 13:64-75

41. Blais JD, Filipenko V, Bi M, Harding HP, Ron D, Koumenis C, Wouters BG, Bell JC: Activating transcription factor 4 is translationally regulated by hypoxic stress, Mol Cell Biol 2004, 24:7469-7482

42. Lang KJ, Kappel A, Goodall GJ: Hypoxia-inducible Factor-1alpha mRNA Contains an Internal Ribosome Entry Site That Allows Efficient Translation during Normoxia and Hypoxia, Mol Biol Cell 2002, 13:1792-1801

43. Stein I, Itin A, Einat P, Skaliter R, Grossman Z, Keshet E: Translation of VEGF mRNA by internal ribosome entry: implications for translation under hypoxia, Mol Cell Biol 1998, 18:3112-3119.

44. Akiri G, Nahari D, Finkelstein Y, Le SY, Elroy-Stein O, Levi BZ: Regulation of vascular endothelial growth factor (VEGF) expression is mediated by internal initiation of translation and alternative initiation of transcription, Oncogene $1998,17: 227-236$

45. Miller DL, Dibbens JA, Damert A, Risau W, Vadas MA, Goodall GJ: The vascular endothelial growth factor mRNA contains an internal ribosome entry site, FEBS Lett 1998, 434:417-420.

46. Wouters BG, van den Beucken $T$, Magagnin MG, Koritzinsky $M$, Fels $D$, Koumenis $\mathrm{C}$ : Control of the hypoxic response through regulation of mRNA translation, Semin Cell Dev Biol 2005,

47. Wang $V$, Davis DA, Haque M, Huang LE, Yarchoan R: Differential gene upregulation by hypoxia-inducible factor-1alpha and hypoxia-inducible factor2alpha in HEK293T cells, Cancer Res 2005, 65:3299-3306

48. Belanger AJ, Lu H, Date T, Liu LX, Vincent KA, Akita GY, Cheng SH, Gregory $R J$, Jiang C: Hypoxia up-regulates expression of peroxisome proliferatoractivated receptor gamma angiopoietin-related gene (PGAR) in cardiomyocytes: role of hypoxia inducible factor 1alpha, J Mol Cell Cardiol 2002, 34:765-774

49. Le Jan S, Amy C, Cazes A, Monnot C, Lamande N, Favier J, Philippe J, Sibony M, Gasc JM, Corvol P, Germain S: Angiopoietin-like 4 is a proangiogenic factor produced during ischemia and in conventional renal cell carcinoma, Am J Pathol 2003, 162:1521-1528 
50. Leonard MO, Cottell DC, Godson C, Brady HR, Taylor CT: The role of HIF-1 alpha in transcriptional regulation of the proximal tubular epithelial cell response to hypoxia, J Biol Chem 2003, 278:40296-40304

51. Wykoff CC, Pugh CW, Maxwell PH, Harris AL, Ratcliffe PJ: Identification of novel hypoxia dependent and independent target genes of the von HippelLindau (VHL) tumour suppressor by mRNA differential expression profiling, Oncogene 2000, 19:6297-6305

52. Sonna LA, Cullivan ML, Sheldon HK, Pratt RE, Lilly CM: Effect of hypoxia on gene expression by human hepatocytes (HepG2), Physiol Genomics 2003, 12:195-207

53. Staller P, Sulitkova J, Lisztwan J, Moch H, Oakeley EJ, Krek W: Chemokine receptor CXCR4 downregulated by von Hippel-Lindau tumour suppressor pVHL, Nature 2003, 425:307-311

54. Denko N, Schindler C, Koong A, Laderoute K, Green C, Giaccia A: Epigenetic regulation of gene expression in cervical cancer cells by the tumor microenvironment, Clin Cancer Res 2000, 6:480-487

55. Gleadle JM, Ebert BL, Firth JD, Ratcliffe PJ: Regulation of angiogenic growth factor expression by hypoxia, transition metals, and chelating agents, Am J Physiol 1995, 268:C1362-1368

56. Ito D, Walker JR, Thompson CS, Moroz I, Lin W, Veselits ML, Hakim AM, Fienberg AA, Thinakaran G: Characterization of stanniocalcin 2, a novel target of the mammalian unfolded protein response with cytoprotective properties, Mol Cell Biol 2004, 24:9456-9469

57. Brugarolas J, Lei K, Hurley RL, Manning BD, Reiling JH, Hafen E, Witters LA, Ellisen LW, Kaelin WG, Jr.: Regulation of mTOR function in response to hypoxia by REDD1 and the TSC1/TSC2 tumor suppressor complex, Genes Dev 2004, 18:2893-2904

58. Carriere A, Carmona MC, Fernandez $Y$, Rigoulet $M$, Wenger RH, Penicaud L, Casteilla L: Mitochondrial reactive oxygen species control the transcription factor CHOP-10/GADD153 and adipocyte differentiation: a mechanism for hypoxia-dependent effect, J Biol Chem 2004, 279:40462-40469

59. Marciniak SJ, Yun CY, Oyadomari S, Novoa I, Zhang Y, Jungreis R, Nagata K, Harding HP, Ron D: CHOP induces death by promoting protein synthesis and oxidation in the stressed endoplasmic reticulum, Genes Dev 2004, 18:30663077

60. Minchenko O, Opentanova I, Minchenko D, Ogura T, Esumi H: Hypoxia induces transcription of 6-phosphofructo-2-kinase/fructose-2,6biphosphatase4 gene via HIF1 $\alpha$ activation,FEBS Lett 2004, 576:14-20

61. Yoon DY, Buchler P, Saarikoski ST, Hines OJ, Reber HA, Hankinson O: Identification of genes differentially induced by hypoxia in pancreatic cancer cells, Biochem Biophys Res Commun 2001, 288:882-886

62. Laderoute KR, Calaoagan JM, Knapp M, Johnson RS: Glucose utilization is essential for hypoxia-inducible factor 1 alpha-dependent phosphorylation of cJun, Mol Cell Biol 2004, 24:4128-4137

63. Urano $F$, Wang $X$, Bertolotti $A$, Zhang $Y$, Chung $P$, Harding HP, Ron D: Coupling of stress in the ER to activation of JNK protein kinases by transmembrane protein kinase IRE1, Science 2000, 287:664-666

64. Kurisu J, Honma $A$, Miyajima $H$, Kondo $S$, Okumura $M$, Imaizumi $K$ : MDG1/ERdj4, an ER-resident DnaJ family member, suppresses cell death induced by ER stress, Genes Celis 2003, 8:189-202

65. Hu CJ, Wang LY, Chodosh LA, Keith B, Simon MC: Differential roles of hypoxia-inducible factor 1alpha (HIF-1alpha) and HIF-2alpha in hypoxic gene regulation, Mol Cell Biol 2003, 23:9361-9374 
66. Wang $G L$, Jiang $B H$, Rue EA, Semenza $G L$ : Hypoxia-inducible factor 1 is a basic-helix-loop-helix-PAS heterodimer regulated by cellular $\mathrm{O} 2$ tension, Proc Natl Acad Sci U S A 1995, 92:5510-5514.

67. Marjon PL, Bobrovnikova-Marjon EV, Abcouwer SF: Expression of the proangiogenic factors vascular endothelial growth factor and interleukin-8/CXCL8 by human breast carcinomas is responsive to nutrient deprivation and endoplasmic reticulum stress, Mol Cancer 2004, 3:4

68. Kim JY, Ahn HJ, Ryu JH, Suk K, Park JH: BH3-only protein Noxa is a mediator of hypoxic cell death induced by hypoxia-inducible factor 1alpha, J Exp Med 2004, 199:113-124

69. Nakayama K, Frew IJ, Hagensen M, Skals M, Habelhah $H$, Bhoumik A, Kadoya $T$, Erdjument-Bromage $H$, Tempst $P$, Frappell PB, Bowtell DD, Ronai Z: Siah2 regulates stability of prolyl-hydroxylases, controls HIF1alpha abundance, and modulates physiological responses to hypoxia, Cell 2004, 117:941-952

70. Jiang HY, Wek SA, McGrath BC, Lu D, Hai T, Harding HP, Wang $X$, Ron D, Cavener DR, Wek RC: Activating transcription factor 3 is integral to the eIF2 kinase stress response, Mol Cell Biol 2004, 24:1365-1377

71. Jin K, Mao XO, Eshoo MW, del Rio G, Rao R, Chen D, Simon RP, Greenberg DA: CDNA microarray analysis of changes in gene expression induced by neuronal hypoxia in vitro, Neurochem Res 2002, 27:1105-1112

72. Appelhoff RJ, Tian YM, Raval RR, Turley $H$, Harris AL, Pugh CW, Ratcliffe PJ, Gleadle JM: Differential function of the prolyl hydroxylases PHD1, PHD2, and PHD3 in the regulation of hypoxia-inducible factor, ] Biol Chem 2004, 279:38458-38465

73. Marxsen $J H$, Stengel $P$, Doege $K$, Heikkinen $P$, Jokilehto $T$, Wagner $T$, Jelkmann W, Jaakkola $P$, Metzen E: Hypoxia-inducible factor-1 (HIF-1) promotes its degradation by induction of HIF-alpha-prolyl-4-hydroxylases, Biochem J 2004, 381:761-767 


\section{CHAPTER 5}

\section{Regulation of mRNA translation is a major contributor to hypoxia regulated gene expression}

Submitted for publication.

Twan van den Beucken, Michael G. Magagnin, Barry Jutten, Renaud Seigneuric, Philippe Lambin, Marianne Koritzinsky and Bradly G. Wouters 


\begin{abstract}
Hypoxia is a common feature of solid tumors associated with an aggressive phenotype, resistance to therapy and poor prognosis. Many of these adverse effects are mediated by hypoxia induced changes in protein expression. At the transcriptional level this occurs in large part through stabilisation and activation of the HIF family of transcription factors. In addition, hypoxia has recently been shown to regulate the initiation step of MRNA translation through PERK dependent phosphorylation of eIF $2 \alpha$ and through inhibition of the mRNA $5^{\prime}$ cap binding complex eI4F. Although many studies have investigated transcriptional changes during hypoxia, the contribution of regulation at the translational level is less clear. We used gene profiling to characterize global changes in both total and efficiently translated (polysomal) mRNA in DU145 prostate carcinoma cells subjected to hypoxia for different periods of time. Our data indicate that changes in translation efficiency contribute significantly to both hypoxia induced and repressed genes at all time points examined. The contribution is particularly important for the initial response to hypoxia (2-4 hrs) accounting for the majority of identified genes. The influence of transcription becomes more important after prolonged hypoxia, reaching a maximum after 8 hours. Interestingly, the influence of translation rises again after 16 hours particularly for the set of hypoxia repressed genes. The biphasic contribution of translational regulation on differential gene expression during hypoxia is consistent with the early and transient phosphorylation of eIF $2 \alpha$ followed by a more delayed inhibition of eIF4F. Together, our data indicate that translation influences gene expression during hypoxia on a scale comparable to that of transcription.
\end{abstract}


Microarray analysis of translated mRNA during hypoxia

\section{Introduction}

The microenvironment of solid tumors is characterized by poor and heterogeneous oxygenation ${ }^{1}$. Clinical and experimental evidence associates deficiencies in oxygenation (hypoxia) with tumor development, growth, metastasis and poor response to therapy $y^{2-4}$. Activation of multiple cellular adaptive pathways is essential to tolerate hypoxic stress and likely forms the basis for these adverse effects on tumor biology $\mathrm{y}^{5,6}$. Insight into the regulation of these pathways is essential for the development of more effective anticancer therapies.

A large part of the adaptation to hypoxia has been attributed to gene regulation by the HIF family of transcription factors. This is not surprising as HIF triggers a transcriptional program that involves over 60 putative genes coding for proteins involved in angiogenesis, metabolism and metastasis ${ }^{7}$. HIF-1 is a hetrodimeric transcription factor that consists of HIF- $1 \alpha$ and HIF-1 $1 \beta$. Both subunits are ubiquitously expressed, but HIF$1 \alpha$ is rapidly degraded by the 265 proteasome under aerobic conditions in a PVHL (von Hippel-Lindau protein) dependent manner ${ }^{8}$. When oxygen levels become limited HIF- $1 \alpha$ protein is stabilized, binds to HIF-1 $\beta$ and mediates transcription of target genes like vascular endothelial growth factor (VEGF) and carbonic anhydrase 9 (CA9) by binding to the hypoxia responsive element (HRE) in their promoters ${ }^{7}$.

More recently, two other oxygen-sensitive pathways have been described that mediate important cellular processes during hypoxia9-12. The first is through activation of the unfolded protein response (UPR), a program of transcriptional and translational changes occurring as a consequence of endoplasmic reticulum stress. This causes rapid inhibition of mRNA translation through transient phosphorylation of eukaryotic initiation factor $2 \alpha$ (eIF2 $\alpha$ ) at serine 51 by the ER kinase PERK ${ }^{9}, 12$. Although phosphorylation of eIF $2 \alpha$ is transient, translational repression is maintained by a second pathway that deregulates the 5'cap binding complex eIF4F. This complex consists of the cap-binding protein eIF4E, the scaffolding protein eIF4G and the RNA helicase eIF4A. Exposure to 
hypoxia for periods longer than 4 hours causes disruption of the elF4F complex through the sequestration of eIF4E by $4 \mathrm{E}$ binding proteins (4E$B P S)^{9-11,13}$ and relocalization of eIF4E into the nucleus and/ or $p$-bodies by the $4 \mathrm{E}-\mathrm{T}$ transporter protein ${ }^{9}$.

Paradoxically, the overall down regulation of protein synthesis occurs in concert with the initiation of a transcriptional program that is required for adaptation to hypoxia. This raises the question as whether hypoxia induced proteins demonstrate a selective ability to be synthesized under these conditions. It is known that individual mRNA transcripts are translated into protein with widely differing efficiencies and have different reliance on individual initiation factors. Furthermore, we and others have previously shown that specific mRNA transcripts remain associated with polysomes during hypoxia, indicative of de novo protein synthesis ${ }^{9,14}$. This provides tumor cells with a potential mechanism to respond quickly to changes in their microenvironment. We hypothesize that this might be especially important under circumstances where tumor cells experience short, transient periods of hypoxia due to perfusion changes in the tumor vessels ${ }^{15,16}$.

Preferential translation is mediated by particular RNA elements within the $5^{\prime}$ and $3^{\prime}$ untranslated regions (UTRs) and include internal ribosome entry sites (IRESs) and upstream open reading frames (UORFs) ${ }^{17}$. An IRES is a secondary RNA structure that allows direct binding of the ribosomes to the mRNA transcript ${ }^{18}$. This mode of translation appears to be important during hypoxia as it has been reported for HIF-1 $\alpha$, VEGF and BiP ${ }^{19-21}$. Probably the best example for regulation of gene expression by UORFs is ATF-4. This transcription factor is translationally induced by ER stress in spite of PERK-dependent eIF $2 \alpha$ phosphorylation. Translation of ATF-4 is prevented under normal conditions by the presence of UORFs in its $5^{\prime} U T R$. When eIF $2 \alpha$ becomes phosphorylated initiation is less efficient, allowing ribosomes to read through the false UORFs and engage initiation at the proper start codon ${ }^{22}$. Changes in translation of specific genes under hypoxic conditions can also be regulated by a small class of non-coding 


\section{Microarray analysis of iranslated mRNA during hupoxia}

RNAs, called microRNAs (miRNAs) ${ }^{23-25}$. Although most currently known miRNAs repress gene specific mRNA translation, there is one report suggesting that they may also stimulate translation of specific transcripts as well ${ }^{26}$.

Several recent studies have investigated the effect of translational control during hypoxia on a genome-wide scale $\mathrm{e}^{14,27-29}$. By microarray analysis Blais et al. identified several genes, including ATF4, that displayed increased translation efficiency following exposure to 16 hours of hypoxia ${ }^{28}$. Similar experiments have also been conducted after 4 hours of exposure to severe hypoxia ${ }^{14}, 29$, and 20 hours of more moderate hypoxia $\left(1.0 \% \mathrm{O}_{2}\right)^{27}$. Although these studies focused on a single time point after hypoxic exposure, they clearly demonstrate that preferential translation does contribute to hypoxia induced gene expression. Nevertheless, it remains unclear to what extent changes in translation efficiency contribute to global changes (up or down) in gene expression. This contribution is expected to vary significantly during hypoxic exposure, given the fact that the pathways mediating translational control operate with different kinetics and influence translation through different mechanisms ${ }^{9}$. To address this issue we assessed the hypoxic translatome over a period of 2-24 hours of hypoxic exposure and compared it with changes in transcription over a similar period. Our results indicate that translational control is a dynamic but significant contributor to changes in gene expression on a level comparable to that of transcription.

\section{Materials and methods}

\section{Tissue culture and hypoxic conditions}

Exponentially growing prostate carcinoma cells (DU145) were seeded on glass dishes in DMEM media with $10 \%$ fetal calf serum (FCS) (SigmaAldrich) and transferred to a hypoxic culture chamber (MACS VA500 microaerophilic workstation, Don Whitley Scientific, Shipley, UK). The atmosphere in the chamber consisted of $5 \% \mathrm{H}_{2}, 5 \% \mathrm{CO}_{2}$ and residual $\mathrm{N}_{2}$. 


\section{Chapter 5}

An anoxic atmosphere was ensured by the inclusion of a catalyst in the hypoxic chamber that catalyzed the conversion of $\mathrm{H}_{2}$ with any $\mathrm{O}_{2}$ to $\mathrm{H}_{2} \mathrm{O}$.

\section{Polysomal fractionation and analysis}

Cells were treated with $0.1 \mathrm{mg} / \mathrm{ml}$ cycloheximide $(\mathrm{CHX})$ for $3 \mathrm{~min}$ at $37^{\circ} \mathrm{C}$, washed twice with ice cold PBS/CHX and harvested by scraping in Iysis buffer ( $1 \%$ Triton $X-100,0.3 \mathrm{M} \mathrm{NaCl}, 15 \mathrm{mM} \mathrm{MgCl} 2,15 \mathrm{mM}$ Tris ( $\mathrm{pH}$ $7.4), 0.1 \mathrm{mg} / \mathrm{ml} \mathrm{CHX}, 100$ units RNAse-In (Ambion)) at $4{ }^{\circ} \mathrm{C}$. Nuclei were removed, $200 \mu \mathrm{g} / \mathrm{ml}$ heparin was added and residual debris was removed by centrifugation. The lysate was layered on a $10 \mathrm{ml}$ continuous sucrose gradient (20-50\% sucrose in $15 \mathrm{mM} \mathrm{MgCl}_{2}, 15 \mathrm{mM}$ Tris ( $\mathrm{pH} 7.4$ ), $0.3 \mathrm{M}$ $\mathrm{NaCl}$ ). After $90 \mathrm{~min}$ of centrifugation at 39,000 rpm in an SW41-Ti rotor at $4^{\circ} \mathrm{C}$, the absorbance at $254 \mathrm{~nm}$ was measured continuously as a function of gradient depth in a BioRad Laboratories UV monitor. To assess overall translation efficiency, we integrated the area under the curve that represented mRNA attached to more than one or more than five ribosomes, and compared this to the total area under the curve. Samples were collected in $0.55 \mathrm{M}$ final concentration of guanidinium- $\mathrm{HCl}$, subjected to four precipitation steps (ethanol, sodium acetate and lithium chloride), one phenol-chloroform extraction and one column extraction (RNeasy Mini Kit, Qiagen) with extensive washing in between. RNA quantity and quality was checked using the ND-1000 spectrometer (NanoDrop Technologies) and RNA Nano LabChip kit on the 2100 Bioanalyzer (Agilent), respectively.

\section{Western blotting}

Cells were washed twice with cold PBS and scraped in RIPA lysis buffer. After centrifugation at $16,000 \mathrm{rpm}$ supernatant was boiled in Laemmli buffer for $10 \mathrm{~min}$ and proteins were resolved by SDS-PAGE. After electrophoresis proteins were transferred onto nitrocellulose membranes and subsequently blocked overnight at $4^{\circ} \mathrm{C}$ in PBS containing $0.1 \%$ Tween-20 (PBS-T) supplemented with $5 \%$ milk powder. Membranes were probed for $1 \mathrm{~h}$ with antibodies directed against phosphor (serine 51)- 
Microarray analysis of tramslated mRNA during hypoxäa

eIF2 $\alpha$ (Research Genetics), eIF4E, eIF2 $\alpha$, 4EBP1 (all Cell Signaling Technologies) or $\beta$-actin (Sigma). Bound antibodies were visualized using HRP-linked secondary antibodies (anti-rabbit (Cell Signaling Technologies) and anti-mouse (Sigma)) and ECL luminescence (Pierce).

\section{Affymetrix gene array measurements}

Twenty nanograms of RNA was pooled from three independent experiments, representing total or highly efficiently translated RNA from either aerobic or hypoxic cells. The RNA was processed according to the manufacturer's standard protocol (Affymetrix) for 2-cycle amplification. The biotin-labelled samples were hybridized to HG U133 Plus 2.0 chips. GCOS software (Affymetrix) was used for the absolute call (present, marginal, absent) of 54,675 probe sets. To identify the groups of genes regulated by transcription or translation, we filtered for genes demonstrating more than 3 -fold change at two consecutive time points. This resulted in four datasets, representing induced and repressed genes in the hypoxic transcriptome (total mRNA) and translatome (heavy polysomal $m R N A$ ). The change in translation efficiency ( $\Delta$ T.E.) was calculated as the ratio of polysomal signal to total signal for hypoxic exposure divided by the ratio of polysomal signal to total signal during normoxic exposure.

\section{Results}

To evaluate the overall changes in mRNA translation that occur in the human prostate carcinoma cell line DU145 during hypoxia, we assessed the amount and distribution of ribosome associated mRNA (polysomes). To this end mRNA transcripts isolated from cells exposed to hypoxia for different periods of time were fractionated through sucrose gradients. This technique enables separation of transcripts based on the number of associated ribosomes and thus on their translation efficiency. Typical absorption profiles of fractionated RNA as a function of gradient depth are 


\section{Chander 5}

shown in figure $1 \mathrm{~A}$. The two first peaks represent the $40 \mathrm{~S}$ and $60 \mathrm{~S}$ ribosomal subunits respectively, whereas the subsequent peaks represent mRNAs with increasing number of attached ribosomes. Consistent with previous results, exposure to hypoxia caused a rapid decrease in polysomal mRNA and a corresponding increase in free ribosomal subunits. Overall translation efficiency can be estimated by dividing the integrated area under the absorption profile representing the translated fraction by the total integrated area ${ }^{9}$. Figure $1 \mathrm{~B}$ shows the estimated fraction of ribosomes participating in translation as a function of hypoxic exposure from three independent experiments. The fraction of ribosomes involved in mRNA translation changes from $\sim 75 \%$ to $\sim 45 \%$ within 2 hours of exposure to hypoxia and remains low for at least 24 hours. This repression is even more pronounced (from $~ 50 \%$ to $25 \%$ ) in the fraction of highly efficiently translated mRNAs (associated with five or more ribosomes).

The observed overall inhibition of mRNA translation during hypoxia is caused by at least two independent mechanisms as described previously ${ }^{9}$ 12. The first involves activation of an evolutionarily conserved pathway known as the unfolded protein response (UPR). This causes the immediate translational repression through phosphorylation of eIF $2 \alpha$ by the ERresident kinase PERK ${ }^{12}$. We therefore examined the phosphorylation status of eIF $2 \alpha$ as a function of time in the DU145 cell line. Similar to what has been reported in other cell types, hypoxia causes a transient increase in eIF $2 \alpha$ phosphorylation that peaks around 4 hours after exposure to hypoxia (Figure $2 \mathrm{~A}$ ) followed by a gradual loss of phosphorylation ${ }^{9,12}$. Loss of eIF $2 \alpha$ phosphorylation during persistent ER stress is known to occur as a result of the activation of a negative feedback loop involving upregulation of GADD $34^{30}$. 
A.

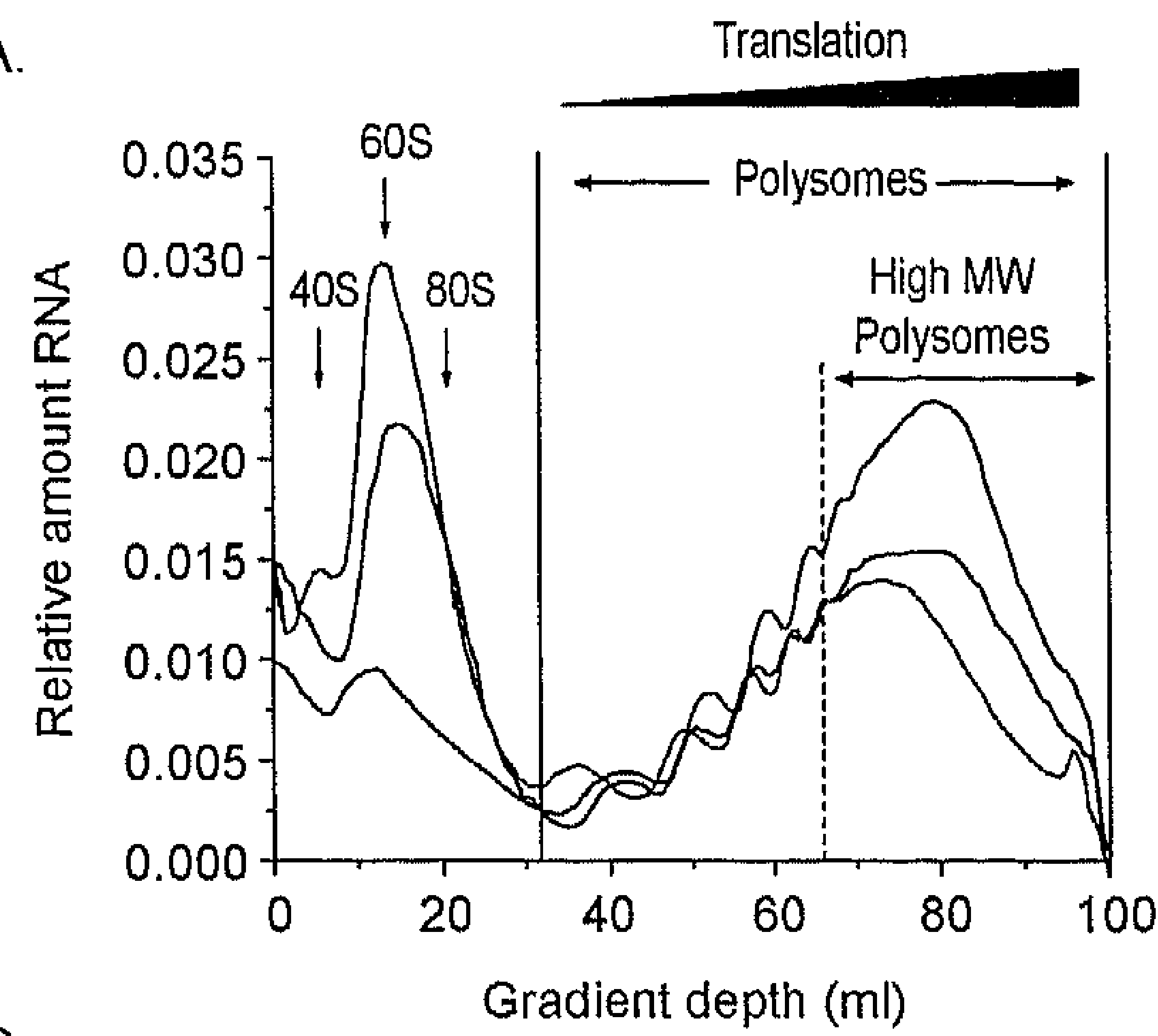

B.

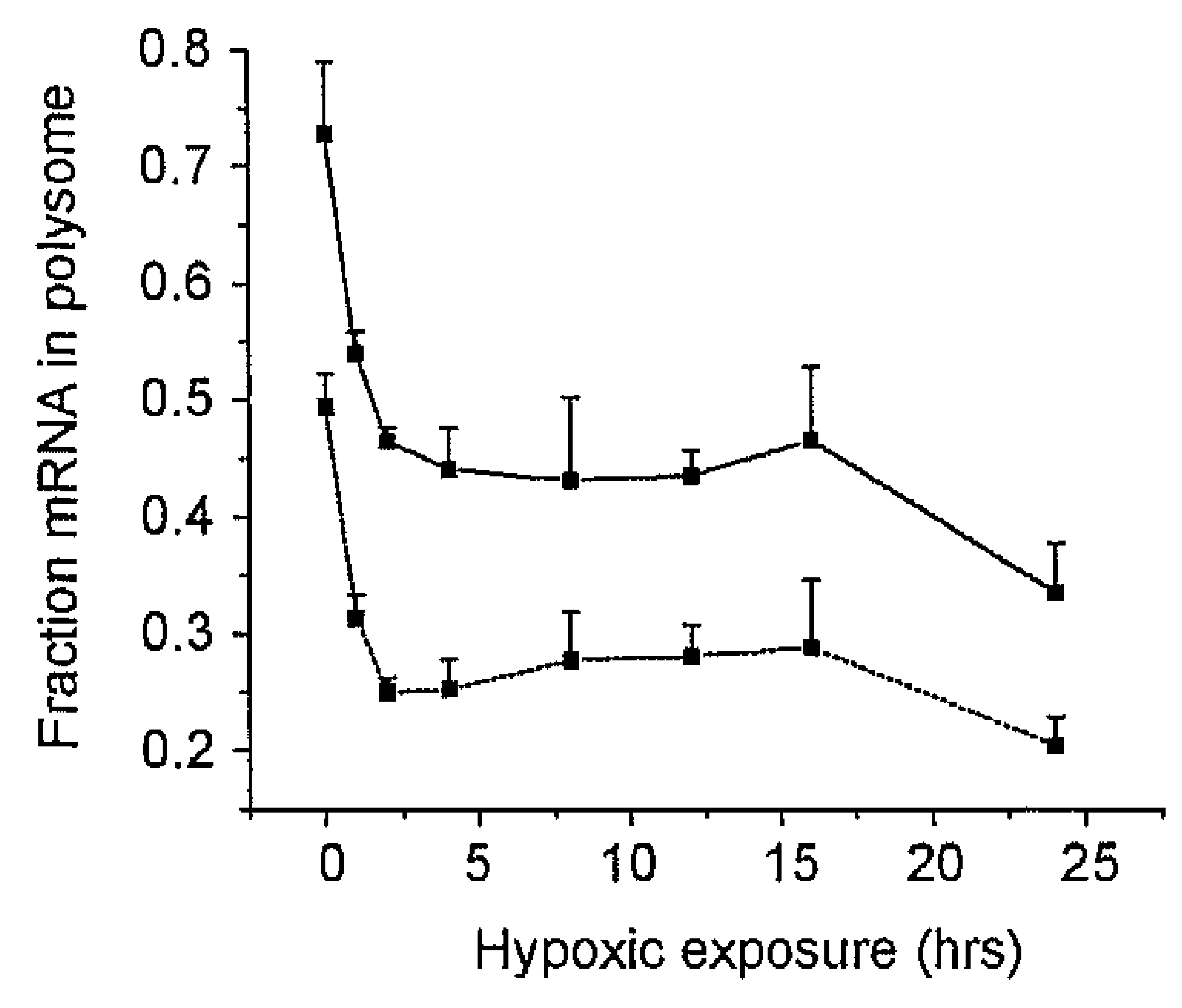

Figure 1. Overall inhibition of mRNA translation during hypoxia. DU145 cells were exposed to $0 \% \mathrm{O}_{2}$ for $0-24$ $h$ and subsequently cell lysates were separated through sucrose gradients. (A) The optical density profiles at $254 \mathrm{~nm}$ as function of gradient depth are shown after hypoxic exposure for 0 (bold), 4 (dotted) and 16 (thin) hrs. (B) Translation efficiency in DU145 as function of exposure time. This was calculated by dividing the integrated area under the curve for the total polysomal (solid line) or high translated polysomal area (dashed line) by the total integrated area.

Longer exposure to hypoxic conditions activates a second mechanism that antagonizes the activity of mammalian target of rapamycin (mTOR) kinase on its target $4 \mathrm{E}-\mathrm{BP} 1^{9,11,13}$. This results in hypo-phosphorylation of $4 \mathrm{E}$ BP1, sequestering of eIF4E and disruption of the 5 'cap binding complex eIF4F. 4E-BP1 appears as three different phosphorylation forms with distinct migration patterns $(\alpha, \beta, \gamma)$ upon Western blotting ${ }^{31}$. Figure $2 B$ shows that under aerobic conditions $4 E-B P 1$ is mainly present in the slow migrating hyperphosphorylated inactive form $(\gamma)$ and that $4 E-B P 1$ is gradually dephosphorylated over 16 hrs of hypoxia, ending up mostly in 


\section{Chapter 5}

its fast-migrating hypo-phosphorylated active form $(\alpha)$. This form of $4 \mathrm{E}-$ BP1 prevents eIF4F assembly by sequestering eIF4E. Protein levels of eIF4E remain unchanged during hypoxia. The data presented in Figure 2 illustrates that both phosphorylation of eIF $2 \alpha$ as well as eIF4F disruption by $4 \mathrm{E}-\mathrm{BP} 1$ contribute to translational repression during hypoxia in DU145 cells.

A

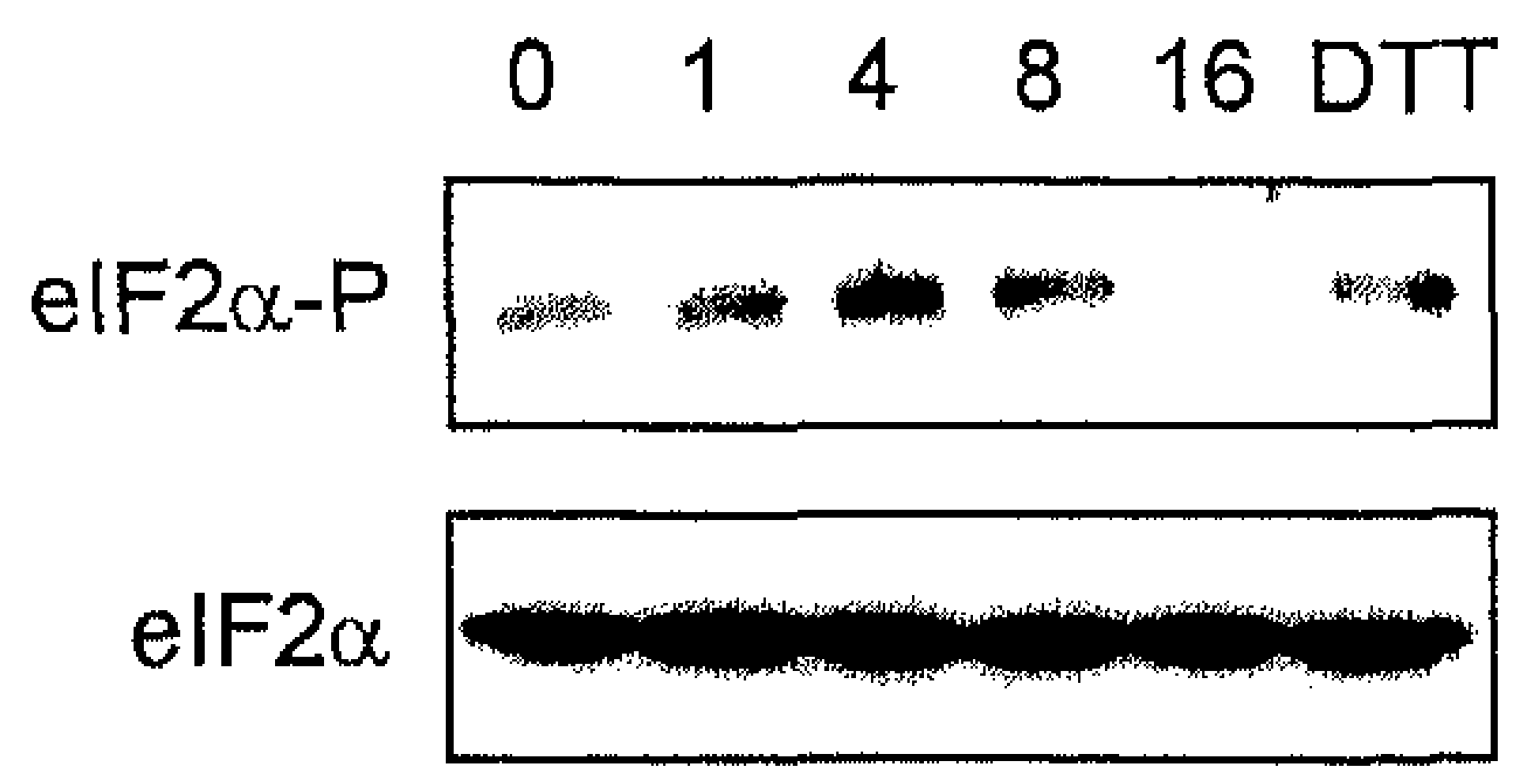

$B$

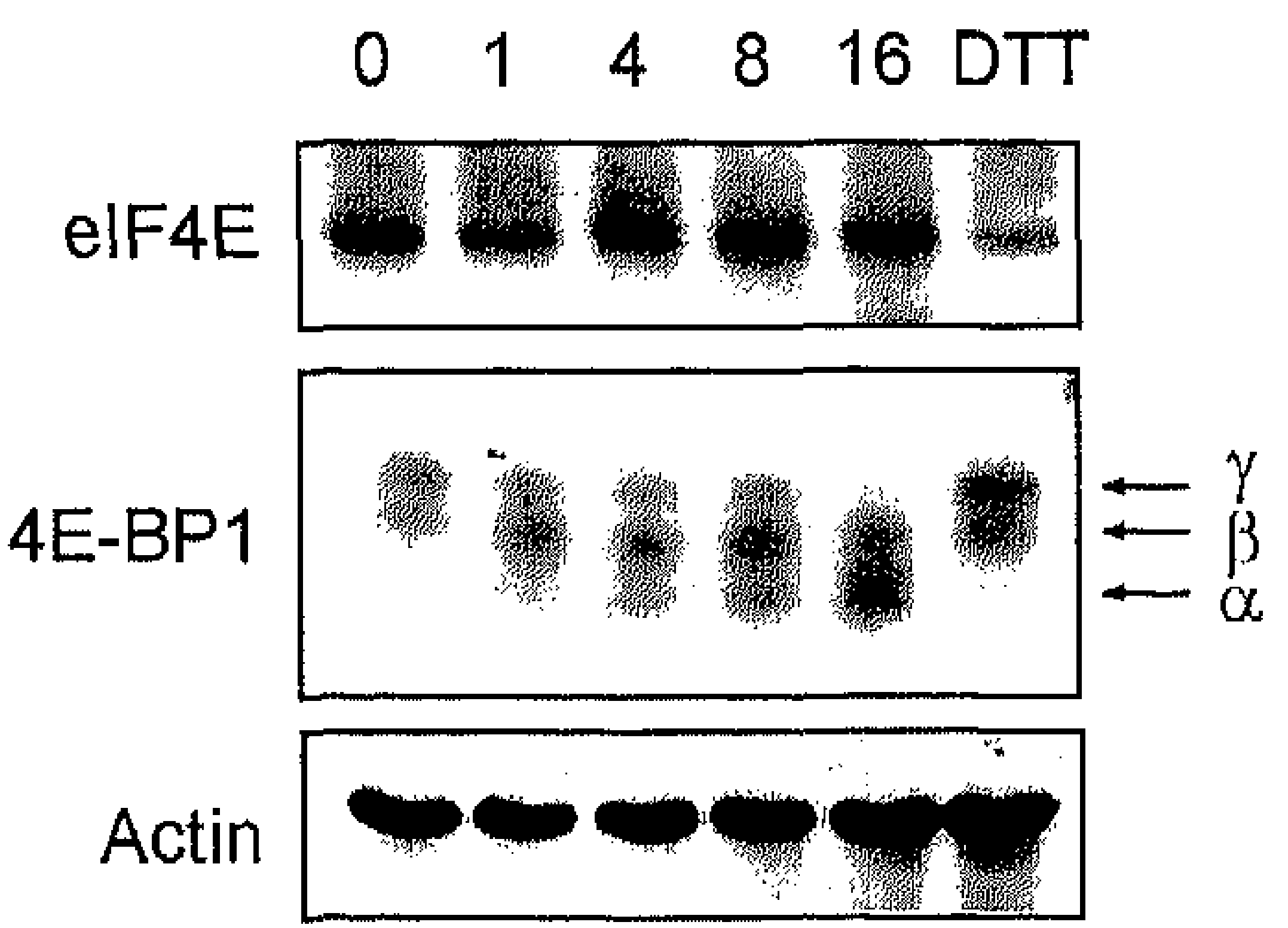

Figure 2. Hypoxia inhibits translation through two molecular pathways. DU145 cells were exposed to $0,1,4,8$ and 16 hrs of $0 \% \mathrm{O}_{2}$ and subjected to Western blot analysis. (A) Phosphorylated eIF2 $\alpha$ with total eIF2a as a loading control. Treatment with $1 \mathrm{mM} \mathrm{DTT}$ for $1 \mathrm{~h}$ was used as a positive control for eIF2 $\alpha$ phosphorylation. (B) 4E-BP1 with eIF4E and actin as loading controls. The SDS page resolves $34 \mathrm{E}-$ BP1 species $(\gamma, \beta, \alpha)$ representing hyper-, intermediate- and hypo-phosphorylated $4 \mathrm{E}-\mathrm{BP} 1$ respectively.

Next we performed a series of gene profiling experiments to determine how regulation of mRNA translation affects hypoxia induced gene expression at the individual gene level. This was done by analyzing mRNA isolated from whole cells (transcriptional changes) or from the heavy polysomal fraction, defined as containing 5 or more ribosomes (referred to as 'translational' changes). Translational changes are influenced both by changes in transcription and by changes in translation efficiency (i.e. the rate at which a specific mRNA is converted into protein) and are closely related to de novo protein production. Transcriptional and translational 


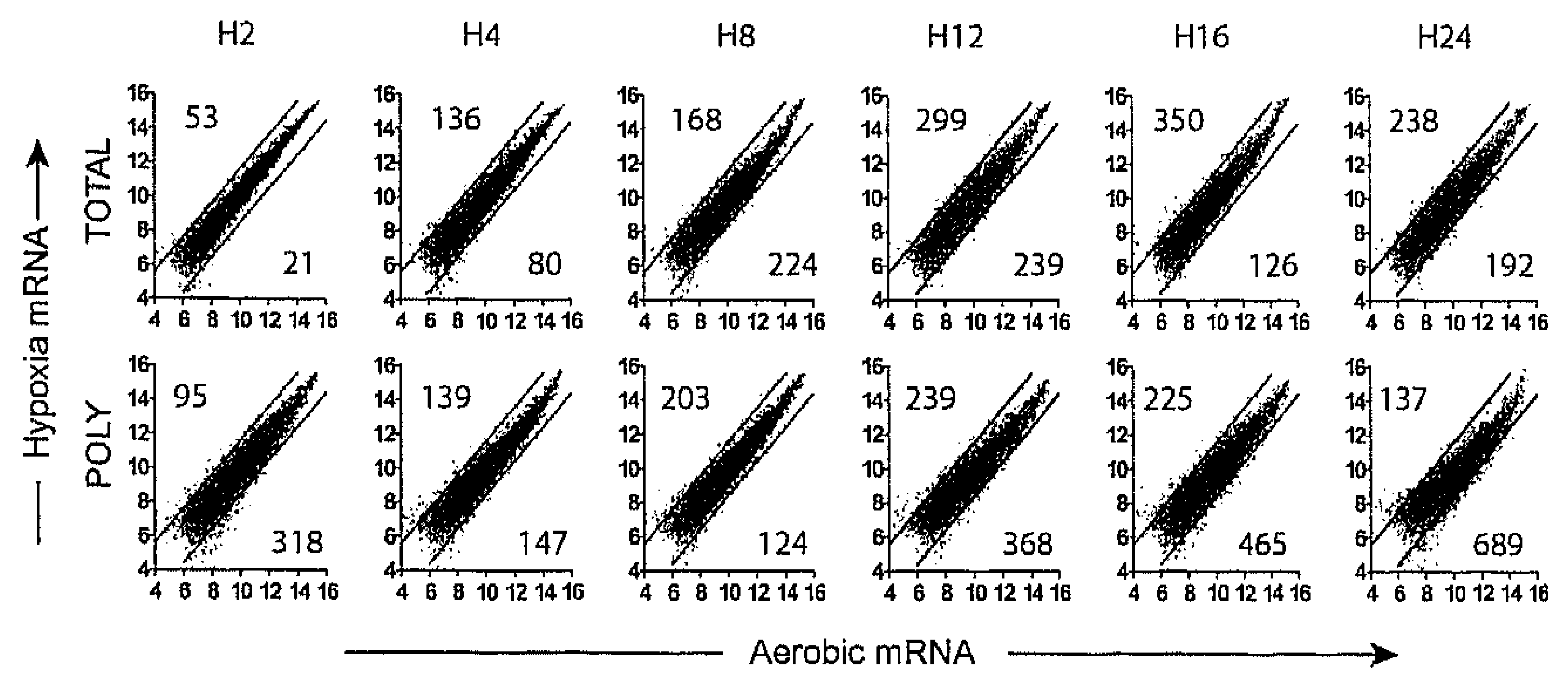

Figure 3. Gene profiling of efficiently translated and total mRNA. Efficiently translated mRNA and total mRNA were isolated from DU145 cells as a function of hypoxic exposure. Equal amounts of mRNA were pooled from 3 independent experiments and hybridized to Affymetrix microarrays. Logarithm of raw intensities for each probe set under hypoxia versus normoxia is shown for total mRNA and efficiently translated mRNA. The number of genes showing more than 3-fold change in expression at two consecutive time points is indicated in each plot. The diagonal upper and lower lines represent threshold for 3 -fold change in expression.

changes were assessed in DU145 cells after exposure to hypoxia for 2, 4, $8,12,16$ and 24 hours. In figure $3 A$ the total and polysomal mRNA levels are shown separately as dotplots for all hypoxic time points versus their respective aerobic control. The data from each array are normalized by the total array intensity such that the total signal for all arrays is equal. Consequently the translation plots do not reflect the average overall inhibition in mRNA translation that occurs during hypoxia as quantified in figure 1B. Instead, these dotplots identify the probes that show significant deviations (up regulated and down regulated) from the overall average inhibition. Visual examination of the dotplots in figure $3 \mathrm{~A}$ suggests that, as expected, changes in transcription between hypoxic and aerobic cells increase as a function of time. This is evident by the fact that total mRNA levels are most similar between hypoxic and aerobic cells at 2 hrs and then diverge for longer exposures. In contrast, by comparing dotplots of poly and total mRNA at 2 hrs, it appears that changes in translation occur earlier than changes in transcription. 


\section{Chapcer 5}

In order to identify a robust set of hypoxia regulated genes at both the transcriptional and translational levels we filtered the data shown in figure 3 for genes demonstrating more than 3 -fold changes in expression at two consecutive time points. The number of genes that fulfill these criteria are indicated in figure $3 \mathrm{~A}$ in each plot. At the transcriptional level, only 53 induced genes are identified after 2 hrs, and this rises steadily with exposure time affecting 350 genes after 16 hrs. The small drop at 24 hrs to 238 genes likely arises from the conditions of our filter since it lacks an additional later consecutive time point to compare with. A consistently smaller number of transcriptionally repressed genes are identified with these filter criteria, where we identified only 21 genes at 2 hrs and as many as 239 at 12 hours. Interestingly, a much larger number of differentially expressed genes are identified at the translational level, particularly for the repressed set of genes. Within 2 hrs we identified 95 up regulated and 318 down regulated genes respectively. After longer exposures the number of genes induced at the translational level was more similar to that for transcription. However, the translationally repressed genes showed a greater dependence on exposure time. The number of genes dropped from 318 to only 124 at 8 hrs and then increased again dramatically reaching as many as 689 at 24 hrs. Consequently, translational control appears to exert a very strong influence on repressed genes during both acute ( 2 hrs) and chronic (16-24 hrs) hypoxic exposure.

Although it is clear from figure 3 that hypoxia regulates different numbers of genes at the transcriptional and translational levels, this figure does not directly address the concordance between the identified gene sets. Differences found between the hypoxia regulated changes in transcription and translation are mediated by potential changes in translation efficiency. In order to provide better insight on this aspect of gene regulation during hypoxia, we analyzed the contributions of transcription and translation efficiency in more detail. 
A

$\begin{array}{lllllll}\text { POLY } & 68 & 84 & 96 & 97 & 72 & 36\end{array}$

$\begin{array}{lllllll}\text { BOTH } & 27 & 55 & 107 & 142 & 153 & 101\end{array}$

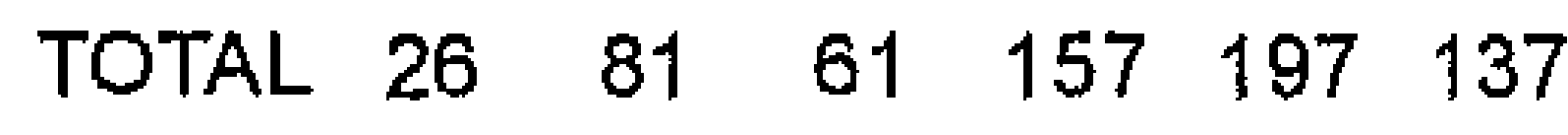

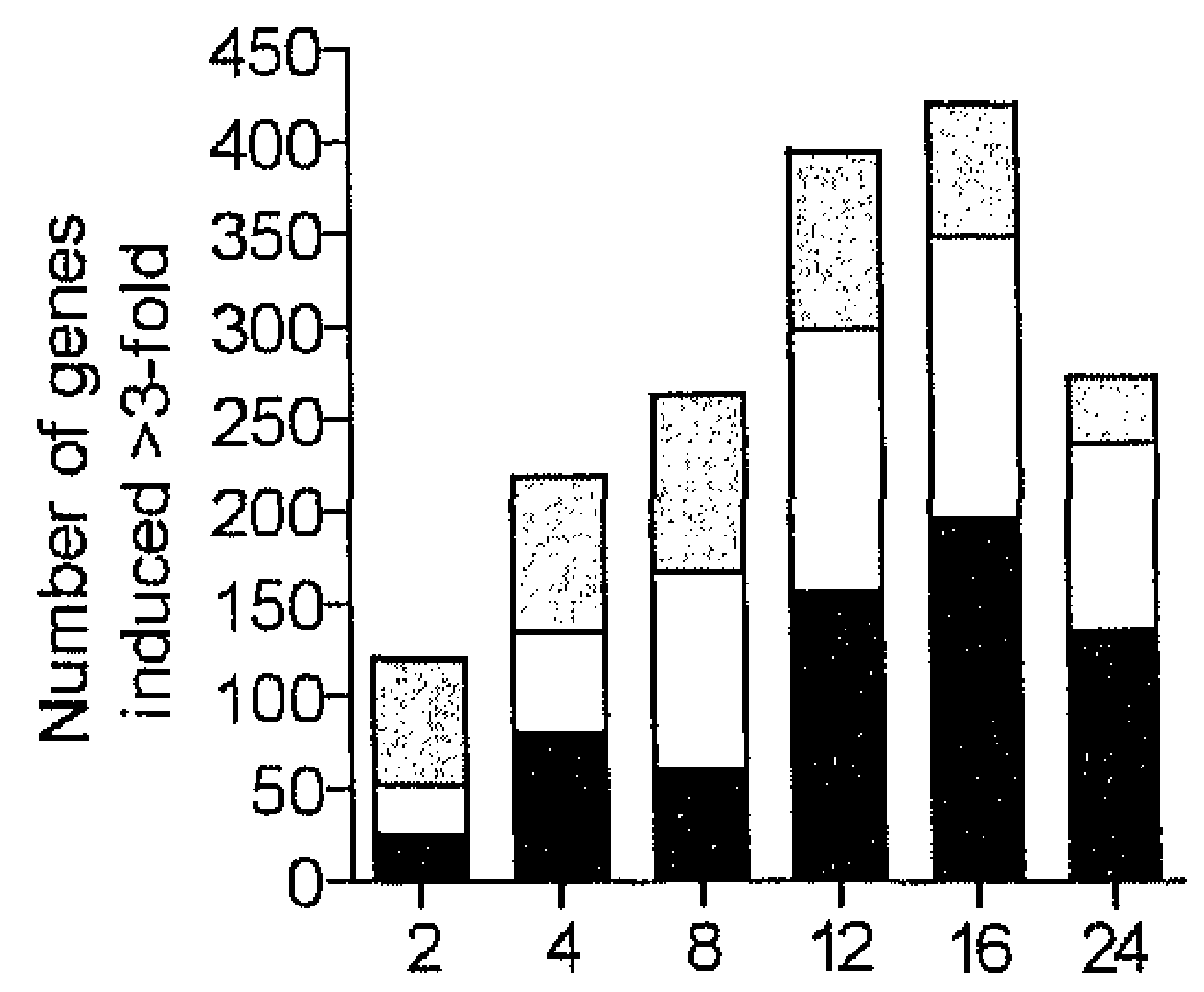

Hypoxic exposure (hrs)

C

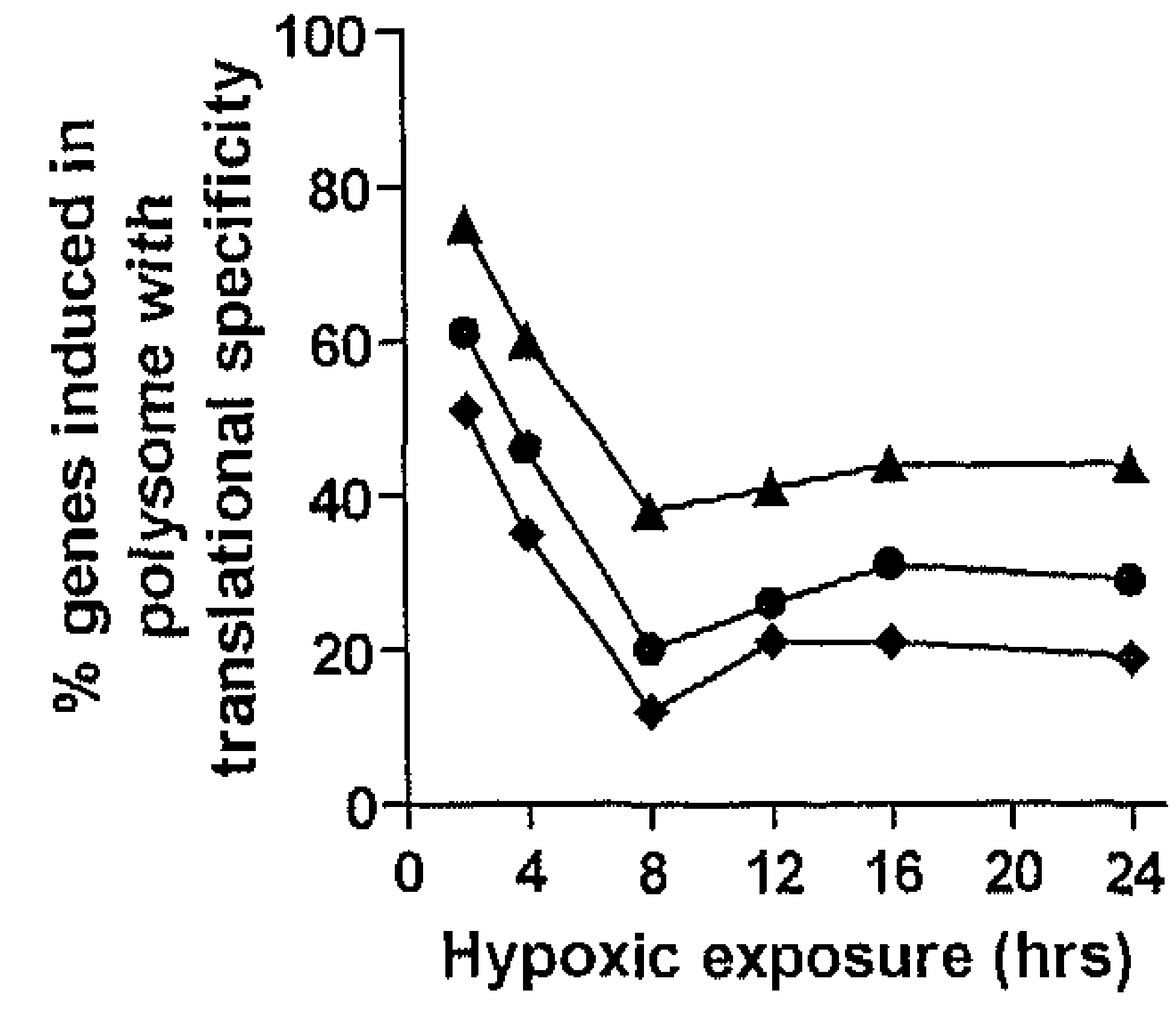

B
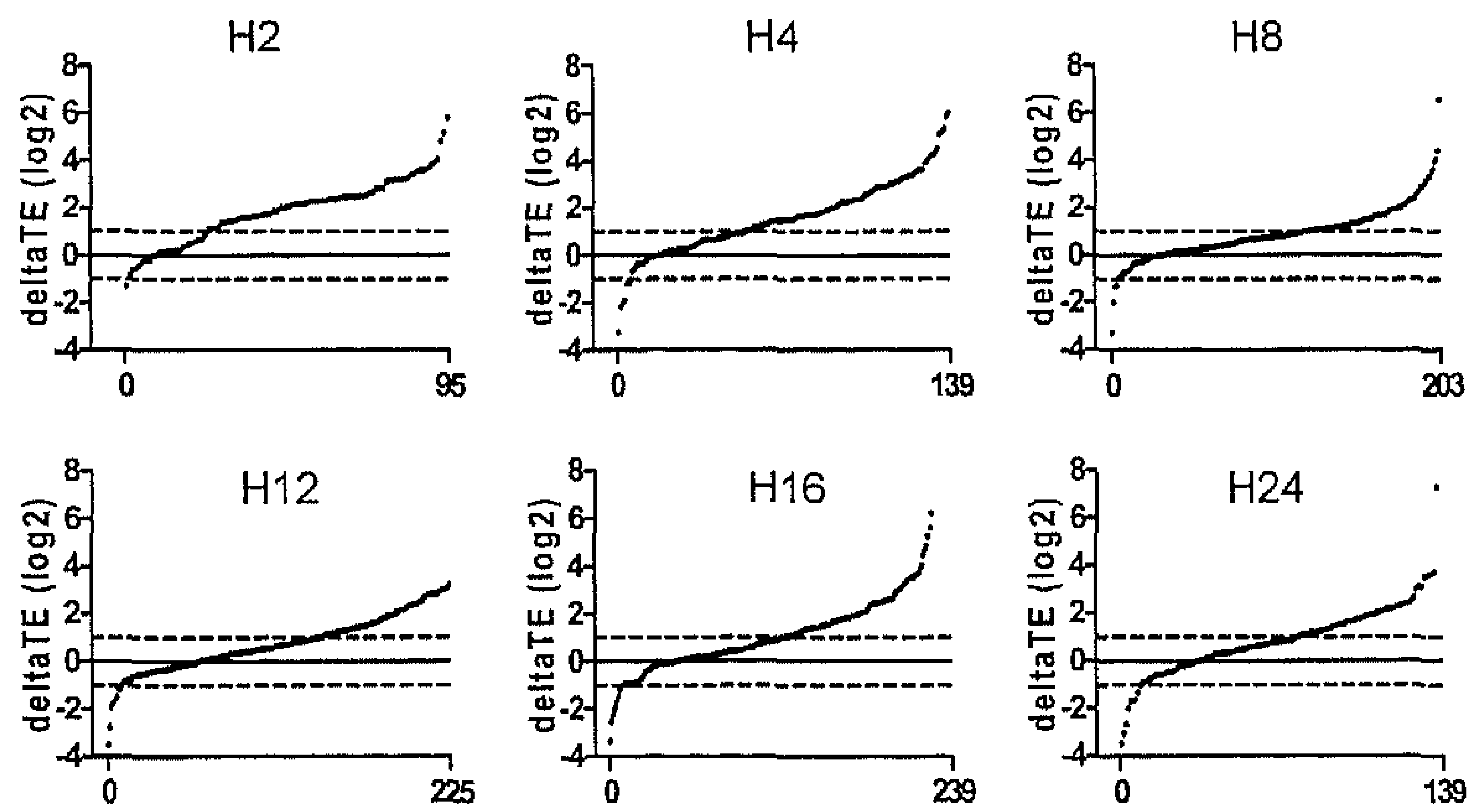

Figure 4. Analysis of hypoxia induced genes. (A) The number of genes showing more than 3-fold increase in expression at two consecutive time points at the total mRNA level (black), the polysomal level (grey) or both (white) were determined and plotted as a function of hypoxic exposure. (B) The changes in T.E. ( $\log 2$ values) were determined as the ratio of polysomal signal to total signal for each gene at the hypoxic time point divided by the same ratio for that gene under aerobic conditions. Threshold levels for no change in TE (solid line) and 2 -fold changes (broken lines) are indicated. (C) The percentage of genes induced in polysomal expression showing preferential translation as a function of hypoxic exposure $\Delta$ T.E. $>2(\Delta), \Delta T . E .>3(\bullet), \Delta T . E .>4(\bullet)$. 
The results of this analysis for induced and repressed genes are shown in figures 4 and 5 respectively.

Figure $4 \mathrm{~A}$ shows the number of genes that were identified only at the transcriptional or translational level as well as those identified in both. Interestingly, the number of genes identified as both transcriptionally and translationally induced is relatively small, with only 27 genes meeting these criteria at 2 hrs. This group of co-regulated genes increases over time to a maximum number of 153 genes after 16 hrs of hypoxia. The percentage of translationally induced genes exhibiting transcriptional coregulation increases from $28 \%$ at 2 hrs to $74 \%$ at $24 \mathrm{hrs}$ of exposure to hypoxia. This is perhaps not surprising as increases in translation arise through both increased transcription and increased translation efficiency of the mRNA.

Despite this overlap, it appears from figure $4 \mathrm{~A}$ that a transcriptional change is frequently not accompanied by a corresponding change at the level of translation and vice versa. This suggests that changes in the translation efficiency of individual genes is contributing significantly to the hypoxia regulated genes found from polysomal mRNA analysis. One potential concern with this analysis is that the lack in overlap between transcriptional and translational identified genes might be caused by the arbitrary threshold values we set to identify the hypoxia regulated genes. To assess this issue more directly we calculated the change in translation efficiency $(\triangle T, E$.$) for each gene in the set of translationally regulated$ genes. The translational efficiency is evaluated by comparing the level of each gene in the polysome to that in the whole cell. As calculated (see methods) $\Delta T$.E. represents the fold change in this number relative to all other genes on the array (i.e. it does not reflect the overall average drop in translation depicted in figure 1). Figure $4 B$ shows the calculated $\Delta T$.E. values, in ascending order, for all translationally regulated genes. At all time points, the majority of genes demonstrate a higher $\Delta T . E$. than the average gene on the array (average is 0 in this dataset) indicating that they are preferentially translated. Both the percentage of genes and the 
degree of increase in $\Delta$ T.E. are highest at early time points ( $2-4 \mathrm{hrs}$ ). In figure $4 \mathrm{C}$, we have plotted the percentage of genes that demonstrate a 2-, 3 - or 4-fold increase in translational efficiency. Nearly 80 percent of the induced genes in the polysome double their T.E. after a $2 \mathrm{hr}$ exposure to hypoxia compared to aerobic conditions and $50 \%$ have an increase of 4fold or more. The contribution of changes in translation efficiency varies with hypoxic exposure time and is maximal at early times when the UPR is activated. The percentage of genes showing this preferential regulation drops to a minimum after 8 hours (less than $20 \%$ showing a 2 -fold increase), and then increases again with a kinetic pattern that is consistent with the disruption of eIF4F. At these later time points it is clear that most of the increases in translation are driven by transcriptional changes.

The same type of analysis was performed on the identified sets of down regulated genes. Figure $5 \mathrm{~A}$ shows the number of genes repressed at the level of transcription, translation or both. It is evident from these data that the changes in translation play an even larger role for repression than for induction. After 2 hours of hypoxia, the expression of over 318 genes is more than 3-fold repressed in the polysomal fraction, this in sharp contrast to the 21 genes repressed at the transcriptional level. Furthermore there is a very limited number of genes identified as transcriptionally and translationally regulated during early hypoxic time points (2-4 hrs). Although a slight recovery in translational repression is observed after 8 hours (124 genes affected), this increases again at late time points affecting a maximum number of 601 genes after $24 \mathrm{hrs}$ of hypoxia. Transcriptional repression is not observed for the majority of translationally repressed genes, with the exception of the $8 \mathrm{hr}$ time point where $71 \%$ of translationally repressed genes are also transcriptionally repressed.

Since transcription did not appear to account for the majority of translationally repressed genes, we also calculated $\Delta T$.E. values for all 


\section{Chapter 5}

A

$\begin{array}{lcccccc}\text { POLY } & 318 & 137 & 36 & 256 & 420 & 601\end{array}$

BOTH $\quad 0 \quad 10 \quad 88 \quad 112 \quad 45 \quad 88$

$\begin{array}{lllllll}\text { TOTAL } & 21 & 70 & 136 & 127 & 81 & 104\end{array}$

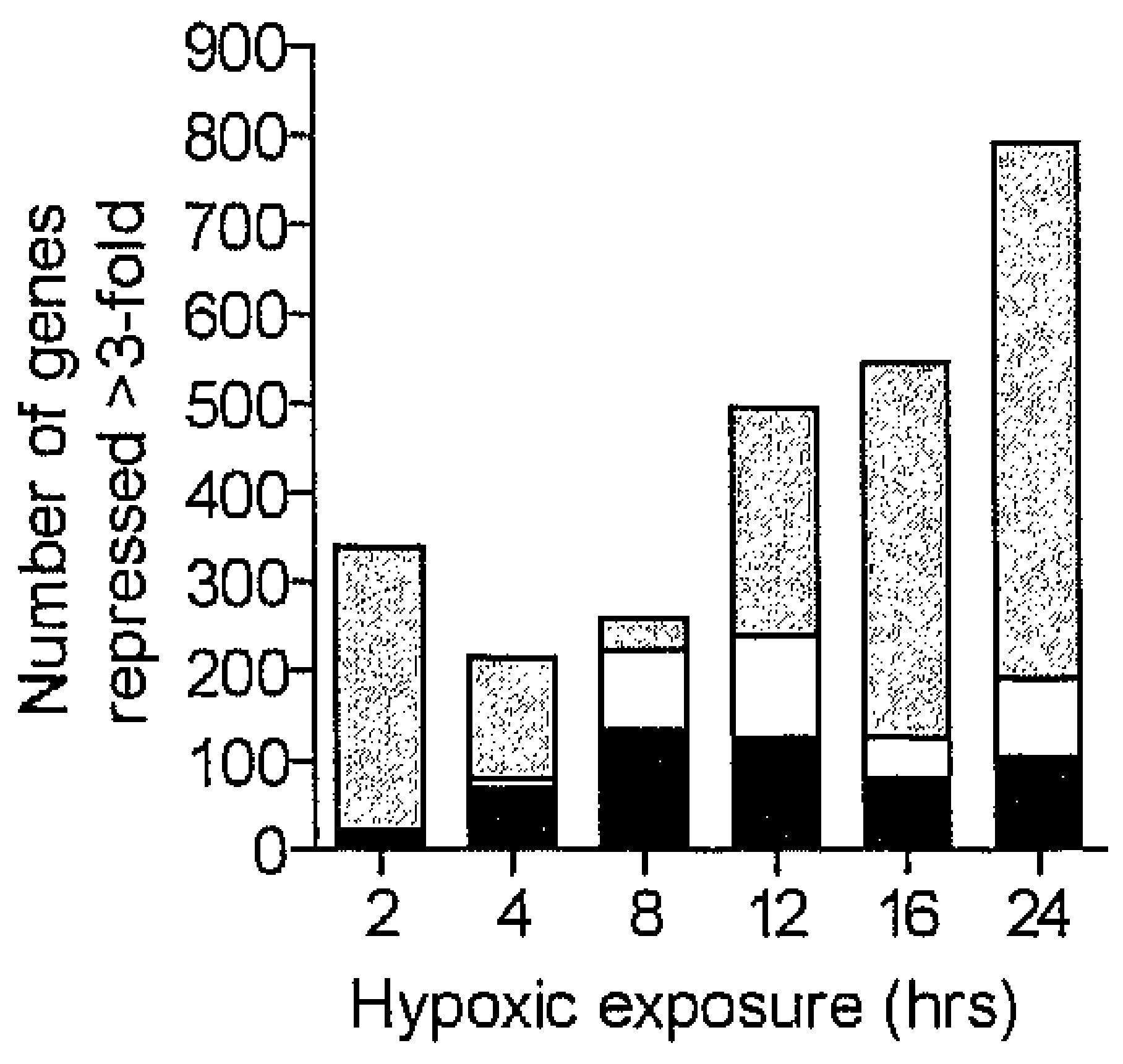

$\mathrm{B}$

$\mathrm{H} 2$

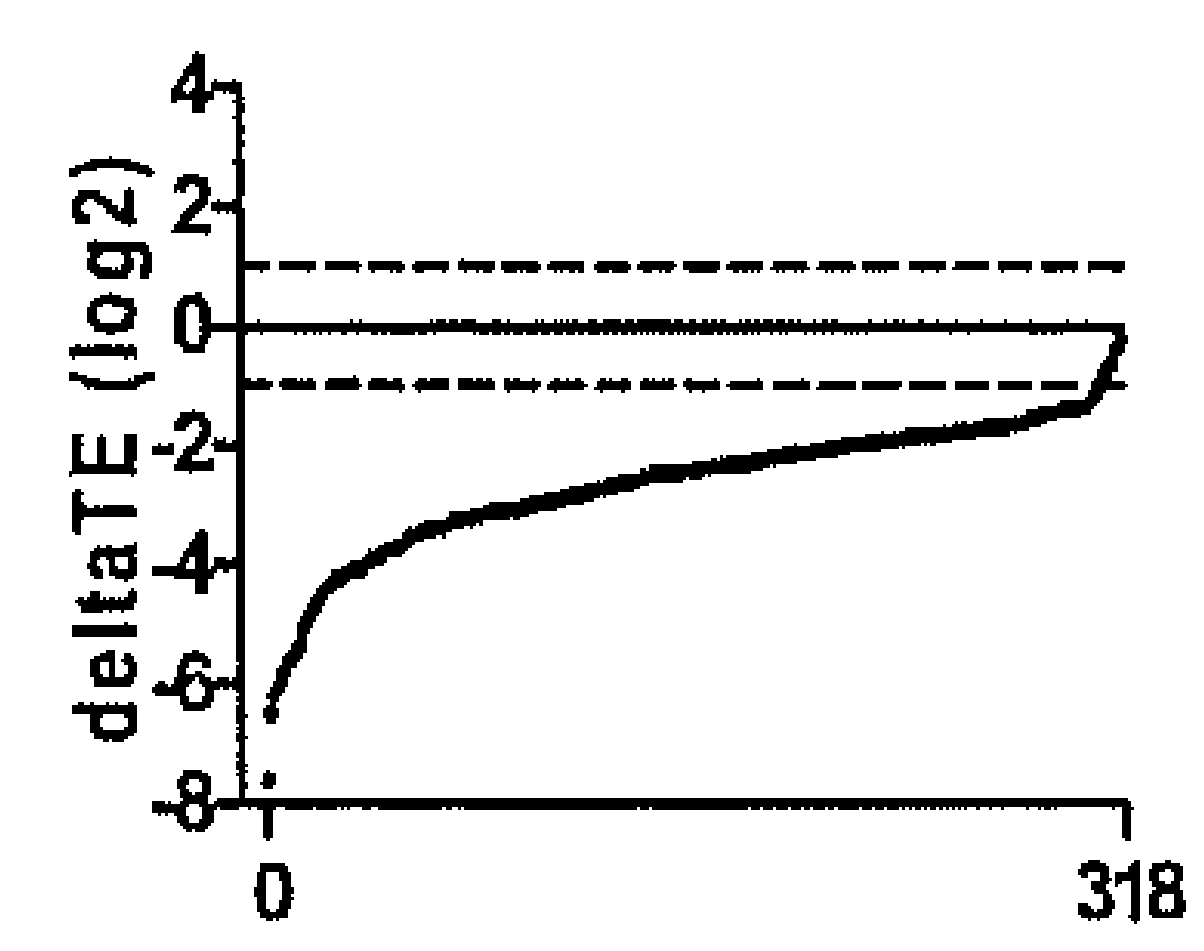

$\mathrm{H} 12$

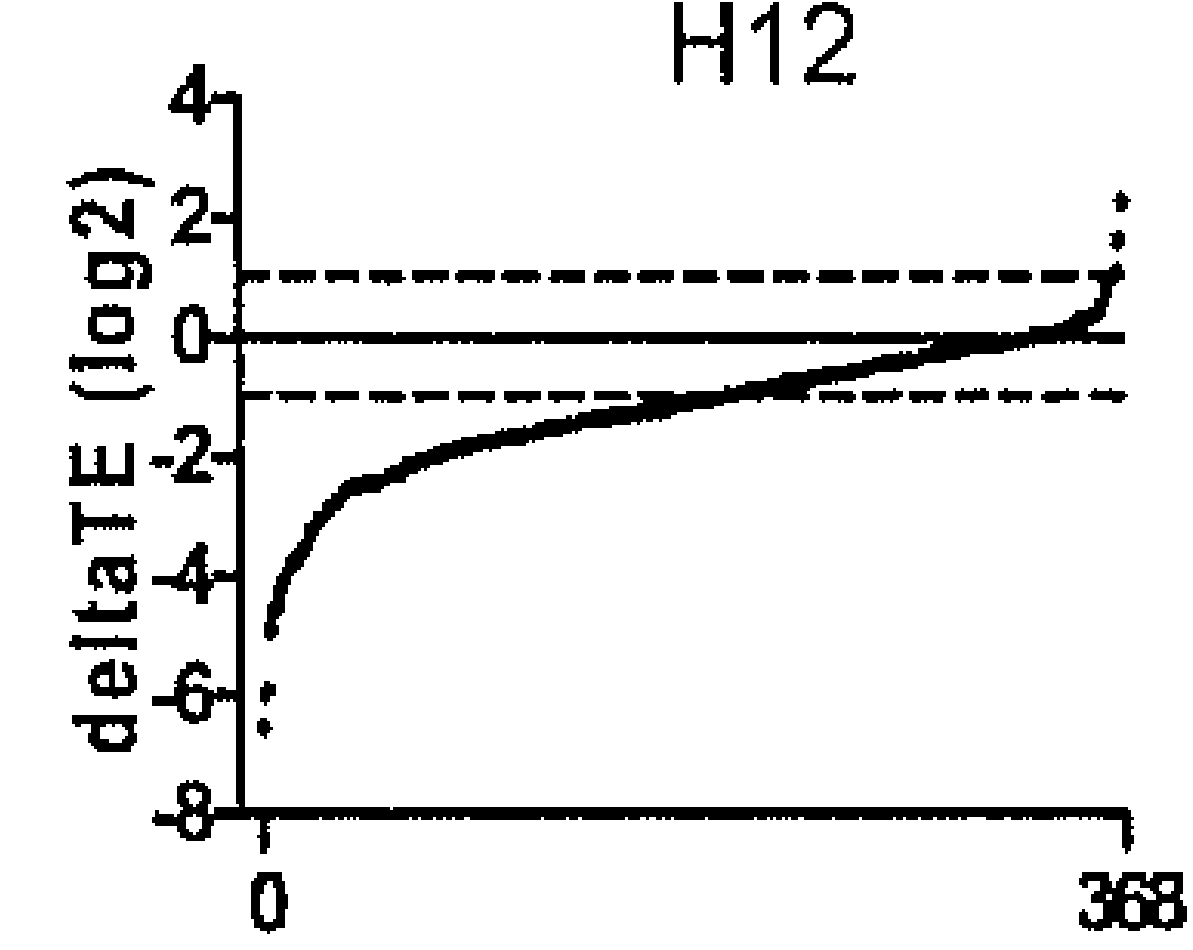

C

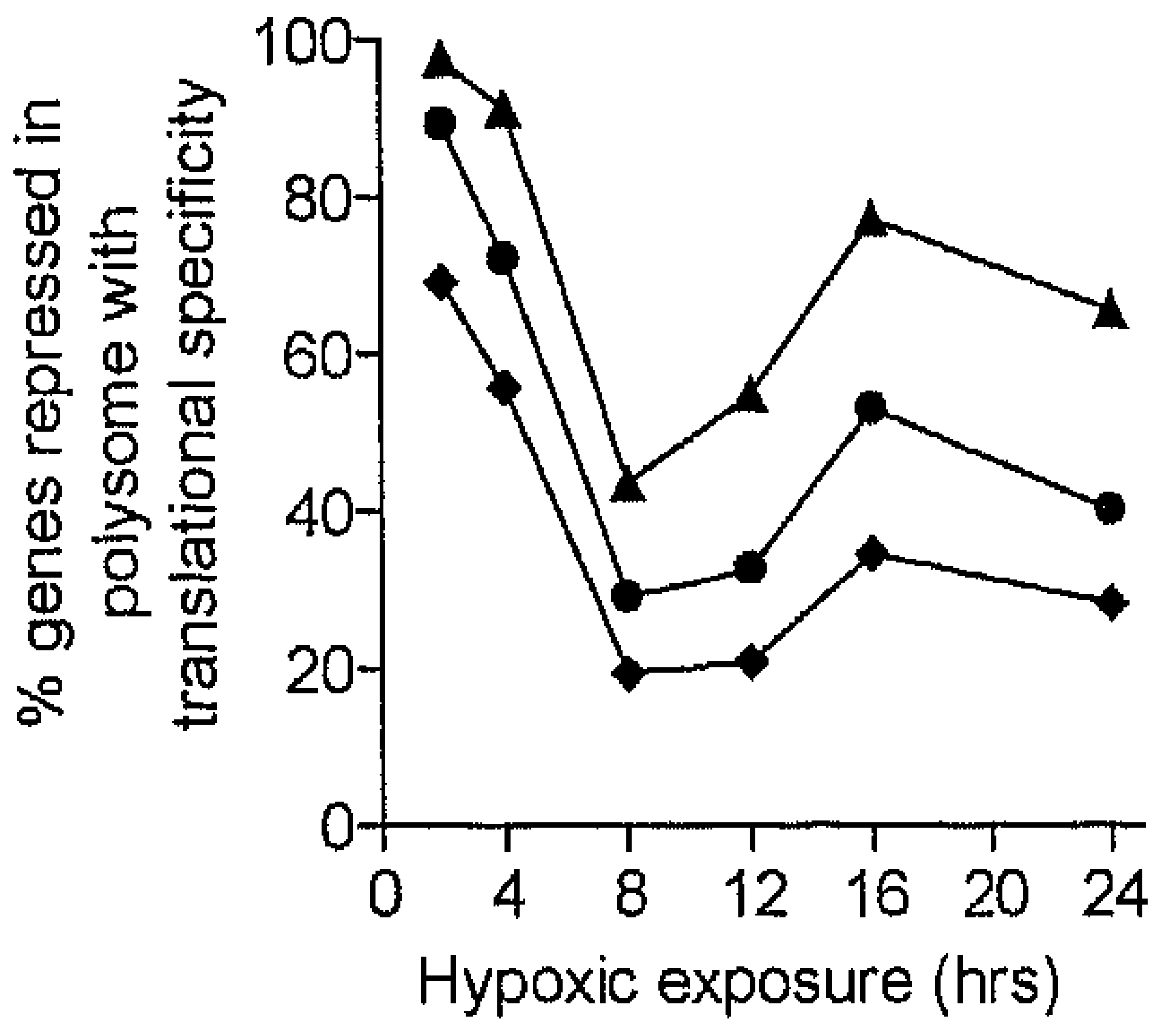

$\mathrm{H4}$

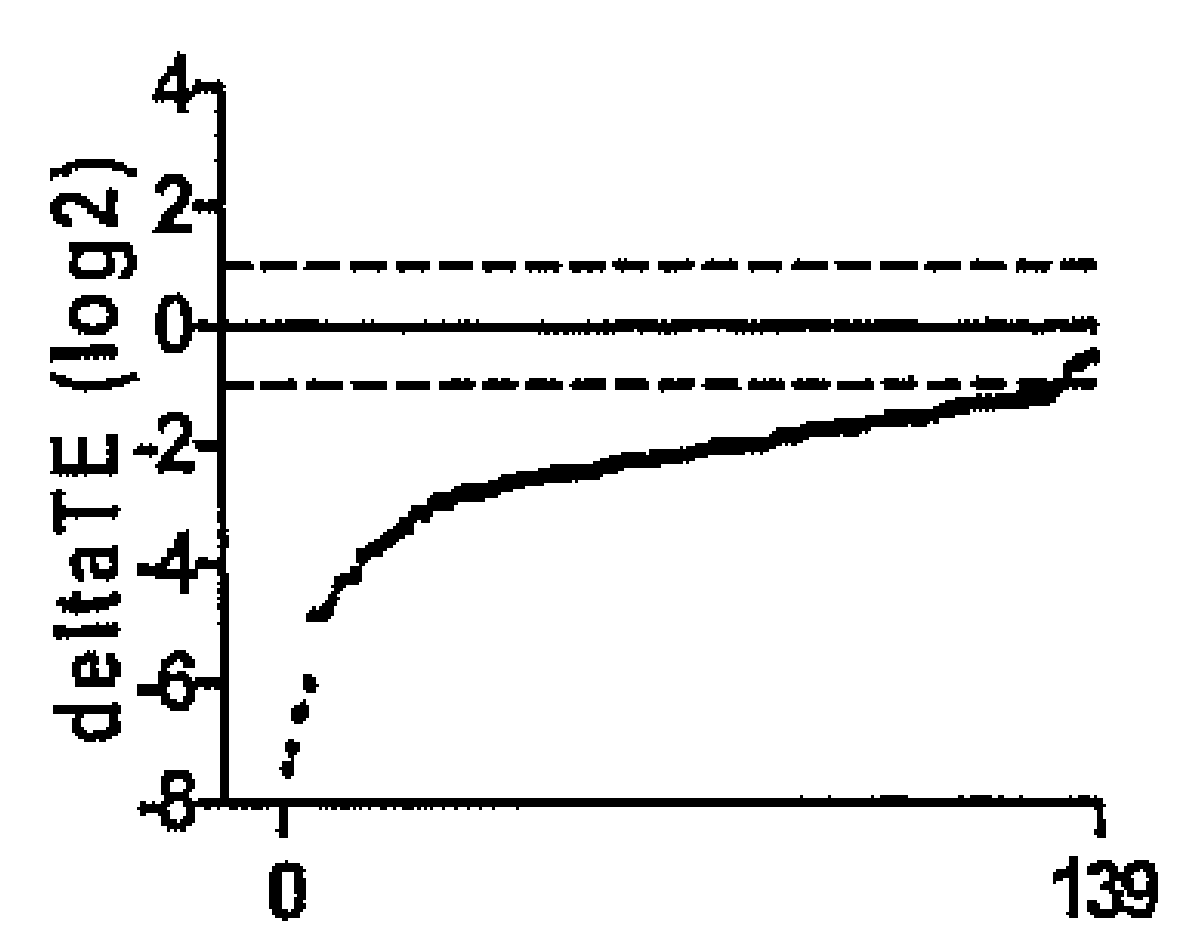

H16

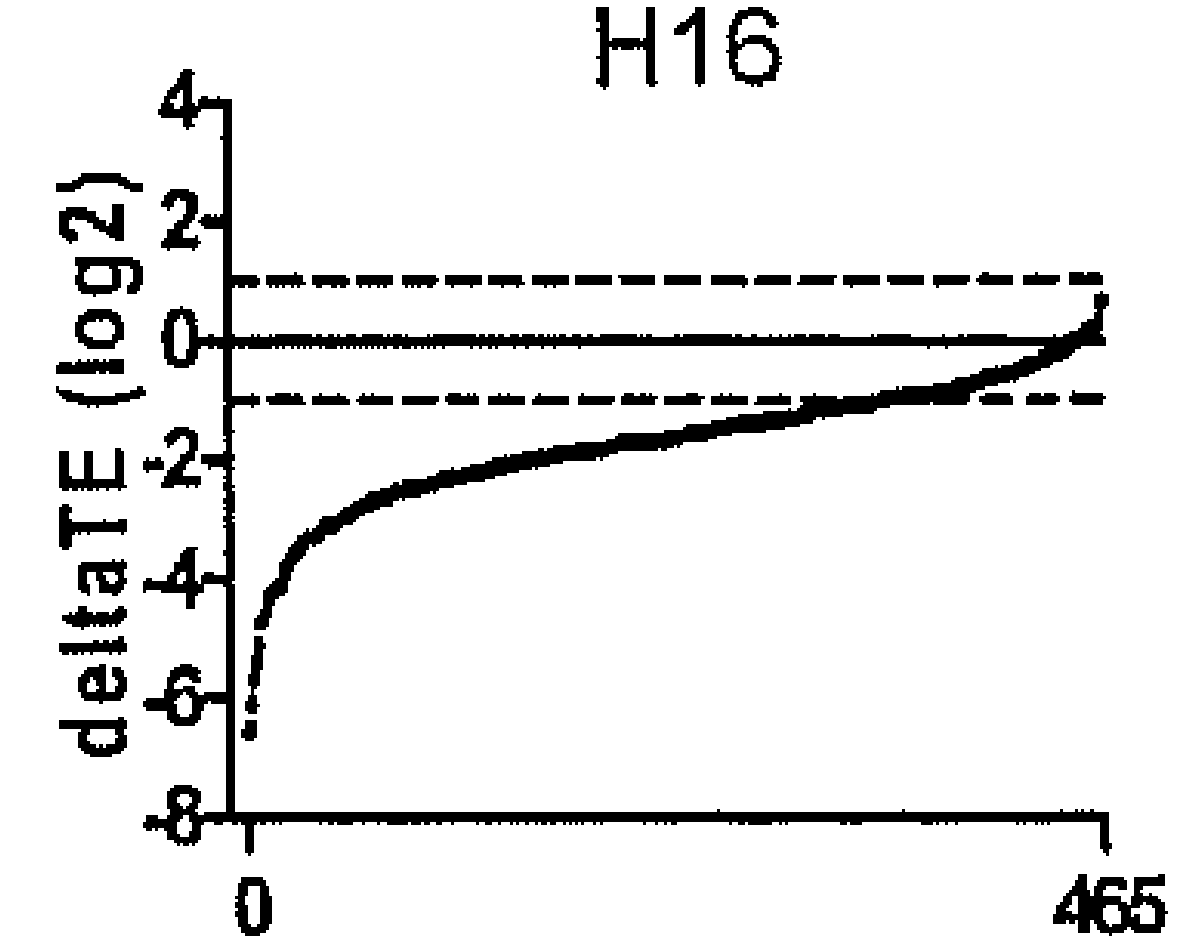

H8

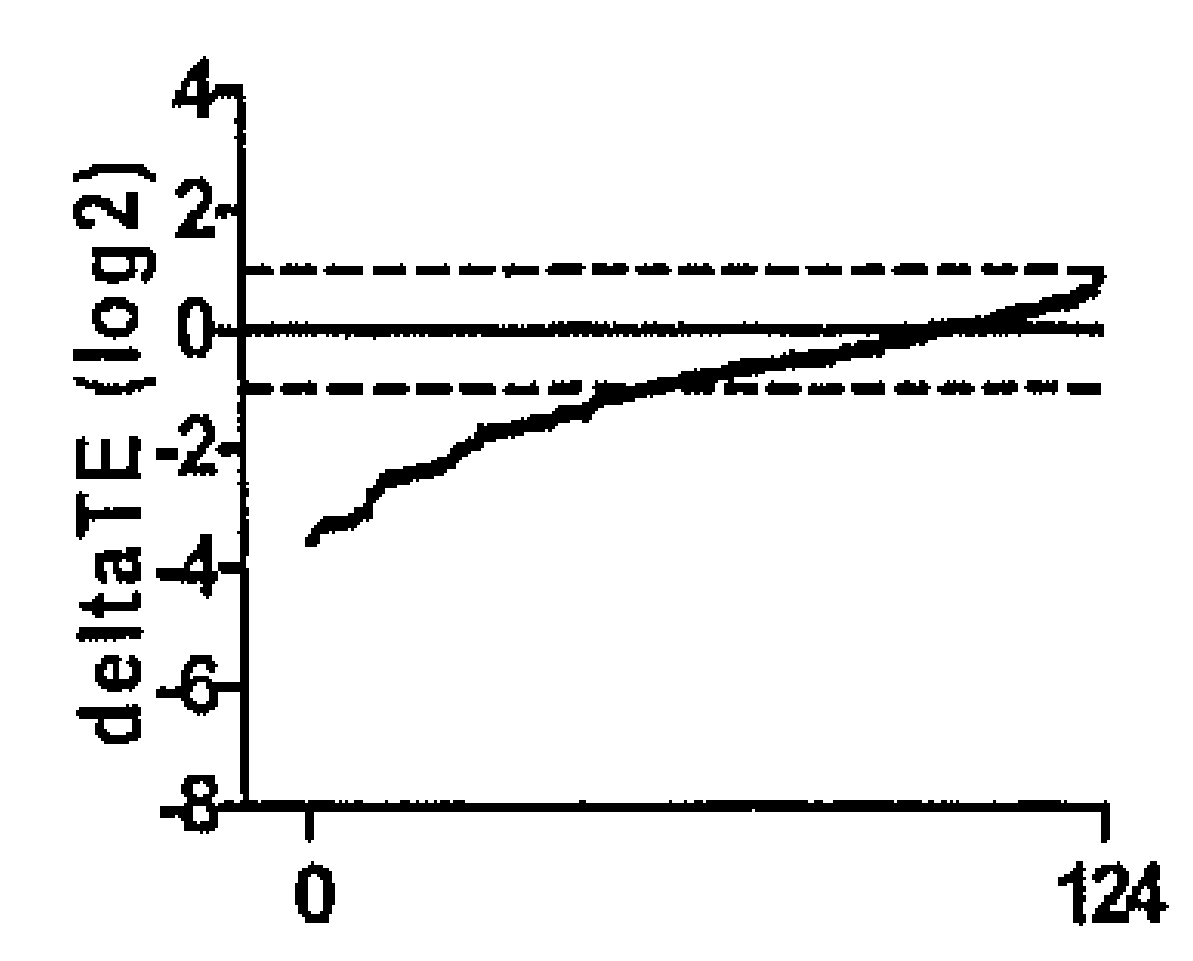

$\mathrm{H} 24$

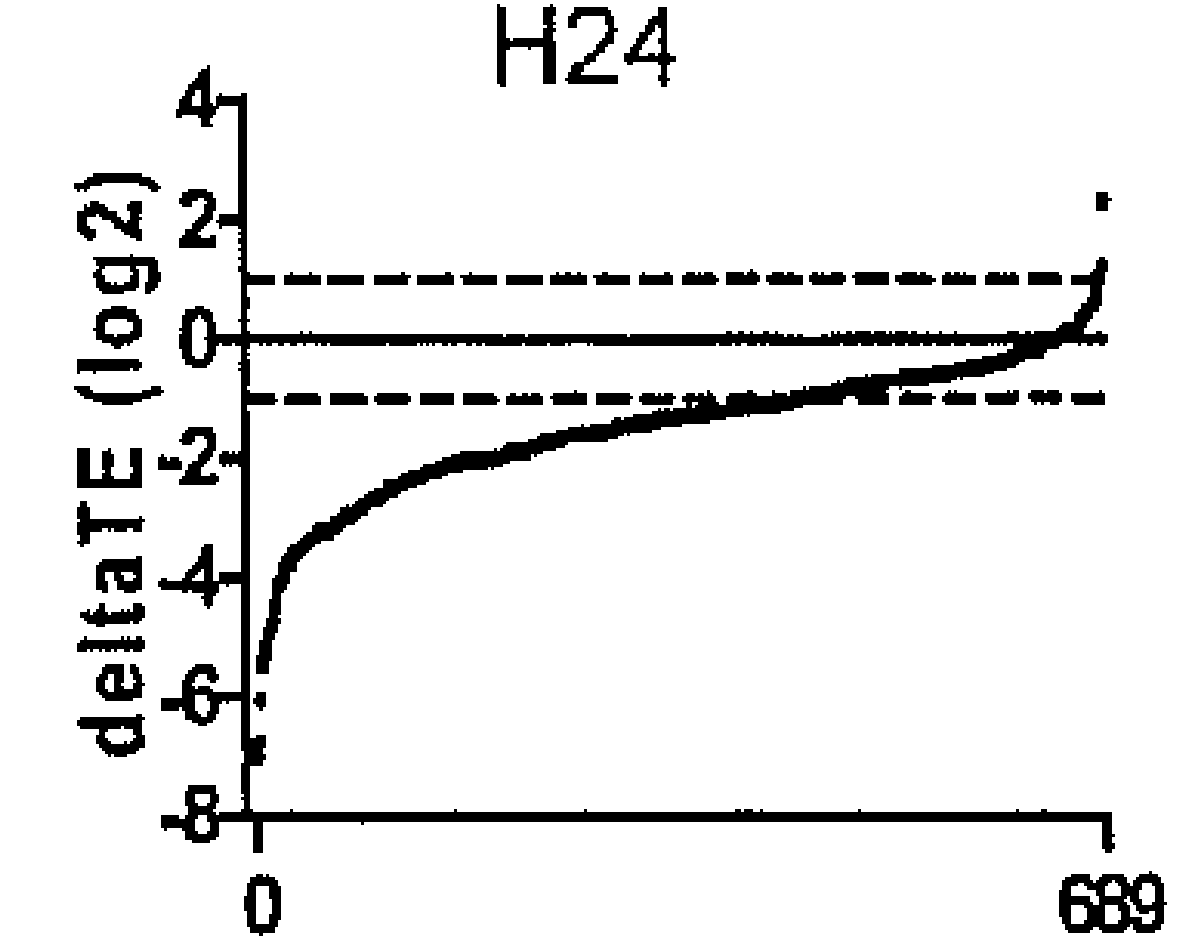

Figure 5. Analysis of hypoxia repressed genes. (A) The number of genes showing more than 3-fold decrease in expression at two consecutive time points at the total mRNA level (black), the polysomal level (grey) or both (white) were determined and plotted as a function of hypoxic exposure. (B) The changes in T.E. (log2 values) were determined as the ratio of polysomal signal to total signal for each gene at the hypoxic time point divided by the same ratio for that gene under aerobic conditions. Threshold levels for no change in TE (solid line) and 2 -fold changes (broken lines) are indicated. (C) The percentage of genes reduced in polysomal expression showing preferential translation as a function of hypoxic exposure $\Delta$ T.E. $<0.5(\Delta), \Delta$ T.E. $<0.33(\bullet), \Delta$ T.E. $<0.25(\diamond)$. 
translationally repressed genes (shown in ascending order in figure 5B). Most of the genes exhibit a lower $\Delta T$.E. than the average gene on the array indicating selective repression. The percentage of genes demonstrating a 2-, 3-, or 4-fold decrease in translation efficiency is shown in figure 5C. After 2 hours of hypoxia, nearly $100 \%$ of the translationally repressed genes demonstrate a $\Delta T$.E. below 0.5 and $70 \%$ even below 0.25 . Similar to the translationally enhanced genes, preferential regulation of the repressed genes reaches a minimum after 8 hours of hypoxic exposure. These kinetics correlate well with transient phosphorylation of eIF $2 \alpha$ at early time points and the disruption of eIF4F at prolonged hypoxia (figure 2). Interestingly, the number of genes that demonstrate large reductions in translation efficiency rises sharply at 16 and 24 hrs consistent with the reduction in cap-dependent translation at these times.

To determine whether translational control influences important biological processes mediated during hypoxia we performed gene ontology (GO) analysis. Enrichment of particular GO terms was determined for both the translationally induced genes ( $>2$-fold induced and $\Delta$ T.E. $>2$ ) and translationally repressed genes ( $>2$-fold repressed and $\Delta T . E .<0.5$ ) at early (2-8 hrs) and late (12-24 hrs) hypoxic time points using the software program EASE ${ }^{32}$. Genes with an EASE score lower than 0.1 were considered to be significantly enriched. Biological themes including transcription, signal transduction and protein transport are selectively affected by translational control during acute hypoxia (table 1). Prolonged exposure to hypoxia mainly influences metabolic processes, although transcription and signal transduction are affected too, albeit to a lesser degree compared to acute hypoxia (table 2). Known hypoxia regulated processes like glycolysis and cell death are also over repressed in the translationally induced genes (data not shown). However these genes do not show an increased change in translation efficiency and are thus not predominantly regulated by translational control. The translationally repressed genes that show significantly reduced mRNA translation rates 


\section{Chapter 5}

include the themes transport, steroid metabolism, cell growth and protein metabolism during acute hypoxia (table 3 ) and protein metabolism, RNA metabolism and mitotic cell cycle during prolonged hypoxia (table 4). Many of the down regulated genes, especially after longer exposures encode proteins that are involved in mRNA translation, including ribosomal proteins $\mathrm{L} 19, \mathrm{~S} 12, \mathrm{~S} 18 \mathrm{~B}$ and $\mathrm{L} 4$ as well as translation initiation factors such as eIF5 and eIF2B.

Table1. Identification of over represented processes in subset of translationally induced genes with $\Delta T$ T.E.>2.0 during acute hypoxia.

\section{Gene Category \\ Nucleobase, nucleoside, nucleotide and nucleic acid metabolism \\ Regulation of DNA- dependent transcription}

\section{Signal transduction}

\section{Protein transport}

Intracellular protein transport

Protein targeting

\section{Phosphorylation}

Protein amino acid phosphorylation

\section{EASE Gene symbols}

1.27E-02 BHLHB2, MXI1, PBX2, CTNNBIP1 AK3, ZBTB1, NFAT5, CITED2, TEAD3, ZNF292, JMJD2B, MFHAS1, HIPK2, C6orf111, SPTBN1, TLE3, PBF

3.94E-03 BHLHB2, MXI1, PBX2, CTNNBIP1, ZBTB1, NFAT5, CITED2, TEAD3, ZNF292, JMJD2B, MFHAS1, HIPK2, TLE3, PBF

4.45E-02 RRBP1, KIF13B, CTNNBIP1, IGF1R, STC1, NFAT5, MFHAS1, SNX27, FL111220, MKNK2, FLJ22570, TLE3, ASB3

4.49E-02 RRBP1, TPR, MXI1, KIF13B, SNX27

3.76E-02 RRBP1, TPR, MXI1, KIF13B, SNX27

1.32E-02 RRBP1, TPR, MXI1, KIF13B

9.98E-02 IGF1R, STC1, HIPK2, MKNK2

7.73E-02 IGF1R, STC1, TAO1, HIPK2, MKNK2, STC1 
Table2. Identification of over represented processes in subset of translationally induced genes with $\Delta$ T.E. $>2.0$ during prolonged hypoxia.

\section{Gene Category}

Metabolism

Protein metabolism

Protein modification

Energy derivation by oxidation of organic compounds

Phosphate metabolism

Protein amino acid phosphorylation

Glutamine family amino acid catabolism

\section{Signal transduction}

Transmembrane receptor protein tyrosine kinase signaling pathway

\section{Transcription}

DNA dependent regulation of transcription

\section{EASE Gene symbols}

score

3.08E-02 RRBP1, RYBP, EGFR, MXI1, WASPIP, KIF13B, IGF1R, ENO3, STC1, ZBTB1, GAD1, DUSP6, TEAD3, PRB1, RUNX1, ZNF292, JMJD2B, TTBK2, SFPQ, NTRK3, LZTS1, FBXL3A, STAT2, PYGB, PBF, KIAA1238, LOC51057, PURG, PTP9Q22, C6orf130

5.13E-02 RRBP, EGFR, MXI1, WASPIP, KIF13B, IGF1R, STC1, GAD1 DUSP6, TTBK2 ,NTRK3 ,FBXL3A KIAA1238, PTP9Q22

6.35E-03 EGFR, IGF1R, STC1, GAD1, DUSP6 , TTBK2, NTRK3, FBXL3A, KIAA1238, PTP9Q22

7.10E-02 ENO3, GAD1, PYGB

3.13E-02 EGFR, IGF1R, STC1, DUSP6, TTBK2, NTRK3, PTP9Q22

9.62E-02 EGFR, IGF1R, STC1, TTBK2, NTRK3

4.45E-02 GAD1, LOC51057

5.69E-02 EGFR, IGF1R, NTRK3

9.95E-02 RYBP, MXI1, ZBTB1, TEAD3, RUNX1, ZNF292, JMJD2B, LZTS1, STAT2, PBF, PURG 
Table3. Identification of over represented processes in subset of translationally repressed genes with $\Delta$ T.E. $<0.5$ during acute hypoxia.

\begin{tabular}{|c|c|c|}
\hline Gene Category & $\begin{array}{l}\text { EASE } \\
\text { score }\end{array}$ & Gene symbols \\
\hline \multicolumn{3}{|l|}{ Transport } \\
\hline $\begin{array}{l}\text { Vesicle-mediated } \\
\text { transport }\end{array}$ & $5.26 \mathrm{E}-03$ & $\begin{array}{l}\text { SNX17, LDLR, SEC23A, SNX4, } \\
\text { HPCAL1, EXOC7, PICALM, SEC15L1 }\end{array}$ \\
\hline Protein transport & $7.42 E-03$ & $\begin{array}{l}\text { M1151, HSPD1, SNX17, SEC23A, } \\
\text { RNP24, SNX4, EXOC7, TIMM22, } \\
\text { SEC15L1 }\end{array}$ \\
\hline $\begin{array}{l}\text { Intracellular protein } \\
\text { transport }\end{array}$ & $5.31 E-03$ & $\begin{array}{l}\text { M11S1, HSPD1,SNX17, SEC23A, } \\
\text { SNX4, TIMM22, SEC15L1 }\end{array}$ \\
\hline Steroid metabolism & $9.04 \mathrm{E}-03$ & LDLR, MVK, OPRS1, APOL2 \\
\hline Sterol metabolism & $7.54 \mathrm{E}-04$ & $\begin{array}{l}\text { LDLR, MVK, HSA9761, OPRS1, } \\
\text { APOL2 }\end{array}$ \\
\hline Cholesterol metabolism & $6.17 E-03$ & LDLR, MVK, APOL2 \\
\hline $\begin{array}{l}\text { Cell growth and/ or } \\
\text { maintenance }\end{array}$ & $8.72 E-03$ & $\begin{array}{l}\text { M11S1, HSPD1, TM4SF8, SNX17, } \\
\text { SLC29A1, LDLR, CDC2, SLC7A6, } \\
\text { RFC3, SEC23A, RNP24, CXCL1, } \\
\text { SNX4, HPCAL1, IL18, TNFSF7, } \\
\text { PSEN1, FSTL1, RFP, SKP2, } \\
\text { HSA9761, ORC5L, CDK2, CCNE2, } \\
\text { EXOC7, DAZAP2, FTSJ1, PICALM, } \\
\text { APOL2, TIMM22, ARPM1, } \\
\text { DKFZP434K0427, CTL1, SEC15L1, } \\
\text { CLASP1 }\end{array}$ \\
\hline Protein metabolism & $2.63 E-03$ & $\begin{array}{l}\text { RPL11, DNAJB1, M11S1, HSPD1, } \\
\text { SNX17, PPIF, PRKAG1, LDLR, CDC2, } \\
\text { SLC7A6, DNAJB4, MVK, STK19, } \\
\text { IL18, CSNK1A1, EIF4EL3, BAG2, } \\
\text { HSPA8, MGAT2, MAP3K7, CDK2, } \\
\text { PICALM, BMP2K, DKFZP761P1010, } \\
\text { MRPL42, EIF3S5, EFG2, USP15, } \\
\text { BMP2K }\end{array}$ \\
\hline Protein folding & $1.30 \mathrm{E}-03$ & $\begin{array}{l}\text { DNAJB1, HSPD1, PPIF, DNAJB4, } \\
\text { BAG2, HSPA8 }\end{array}$ \\
\hline
\end{tabular}


Table4. Identification of over represented processes in subset of translationally repressed genes with $\Delta T$.E. $<0.5$ during prolonged hypoxia.

Gene Category

Protein metabolism

Protein folding

Mitotic cell cycle

Mitosis

RNA metabolism

RNA processing

rRNA processing
EASE

score

2.56E-04 DNAJB1, M11S1, HSPD1, EIF5A, ARIH2, PSMC3, PPIF, ETF1, EIF2B1, CCT2, LDLR, PLK, UBE2S, CDC20, PPP2R1B, CDC2, CAPN7, MRPL19, MAP4K5, UK114, DNAJB4, NUPL2, PPID, MRPS12, MRPS12, FL10613, EIF253, IL18, CDC2L1, PTK2, STK6, EIF5, MALT1, HSPA8, MRPS18B, UFD1L, C2F, BAG2, AURKB, BUB1, CDKN3, PARD3, FKBP1A, HSPA8, CDC2, MGAT2, TGM2, HSPCA, DKFZP564M182, ZRF1, HSPCB, ADAM10, PICALM, TOPK, HSP70-4, FLJ10634, UCHL5, KPNA3, MGC2744, SDPR, HSPC150, USP47, MRPL4, MRPL4, GC14421, CTSC, KIAA1970, CCT5, DNAJA2, NEDL2, EFG1, RARSL, FL12525, MtFMT

9.70E-08 DNAJB1, HSPD1, PPIF, CCT2, DNAJB4, PPID, HSPA8, BAG2, FKBP1A, HSPA8, HSPCA, ZRF1, HSPCB, FLJ10634, CCT5, DNAJA2

9.03E-05 HMGB1, PLK, CDC20, PPP2R1B, CDC2, CCNA2, RFC3, NOLC1, CHC1, CDC2L1, STK6, BUB1, CDKN3, CDC2, ORC5L, MCM4, PRIM2A, TOPK, CCNB1, DNAJA2

1.65E-04 PLK, CDC20, CDC2, CCNA2, NOLC1, CHC1, CDC2L1, STK6, BUB1, CDC2, TOPK, CCNB1

7.45E-07 HSPA1A, HNRPF, DKC1, SFPQ, SFRS1, CCT2, PPP2R1B, SFRS3, RNU3IP2, NOLC1, SFRS6, HNRPH3, CDC2L1, C2F, HSA9761, SR140, SFRS5, POP1, HNRPH1, RRP41, MGC2744,THOC4, WDR4, KIAA1970, RARSL, CPSF2

7.87E-07 HNRPF, DKC1, SFPQ, SFRS1, CCT2, PPP2R1B, SFRS3, RNU3IP2, NOLC1, SFRS6, HNRPH3, CDC2L1, C2F, HSA9761, SR140, SFRS5, POP1, HNRPH1, RRP41, MGC2744,THOC4, WDR4, KIAA1970, RARSL, CPSF2

6.26E-04 DKC1, RNU3IP2, NOLC1, C2F， HSA9761, RRP41 


\section{Chapter 5}

\section{Discussion}

It is becoming increasingly clear that translational control mechanisms play key roles in regulating gene expression that are relevant in cancer. This includes regulation of genes in response to a wide range of cellular stresses such as oncogene activation ${ }^{33}$, radiation ${ }^{34}$ and hypoxia ${ }^{14,} 27,28$. These studies have clearly shown that a substantial number of genes can be preferentially translated during stressful conditions, allowing for rapid changes in protein expression. This effect may be particularly important during hypoxia, which has emerged as a strong regulator of the initiations step of mRNA translation by virtue of its ability to regulate both eIF4E and eIF2 $\alpha$. What remains unclear however, is the extent to which translational mechanisms contribute to differential gene expression compared with that of transcription. To address this point we have studied the effect of hypoxia on gene expression in detail as a function of time and determined the relative contributions of transcriptional and translational responses.

Here we show that mRNA translation is rapidly repressed in DU145 human prostate carcinoma cells during hypoxia. This inhibition is similar to what has been reported for other cell lines" ${ }^{9}$. We furthermore demonstrate that two previously identified mechanisms involved in translational repression during hypoxia, activation of the UPR and inhibition of mTOR are also activated in DU145 cells ${ }^{9-13}$ albeit with different kinetics. Rapid but transient phosphorylation of eIF $2 \alpha$ is followed by the much slower dephosphorylation of 4E-BP1 causing the disruption of the eIF4F complex.

An important aspect of the large decrease in overall rates of translation is its contribution to maintenance of energy homeostasis since protein synthesis in one of the most energy consuming processes within the cell ${ }^{35}$. A second aspect is that this allows the cell to 'reprogram' the translation state of available mRNA transcripts. All available initiation factors can selectively assemble onto different mRNA species, ensuring the translation of important transcripts needed for the adaptation to hypoxic stress. Our group has previously shown for a small number of genes that regulation of 
Microarray analysis of translated MRNA durimg hypoxia

eIF $2 \alpha$ and eIF4F has dramatically different effects on gene specific translation ${ }^{9}$. To obtain a broader overview of this effect we assessed transcriptional and translational changes at different time points during hypoxia on a genome-wide scale. Our results indicate that translational control plays a key role in the up regulation of genes during acute hypoxia, whereas transcriptional control is dominant after prolonged hypoxic exposure. After 2 hours of exposure to hypoxia only $28 \%$ of the translational induced genes show signs of transcriptional co-regulation, whereas this increases over time to $74 \%$ after 24 hours. Interestingly, translational control appears to be the main mechanism for down regulation of gene expression during hypoxia. Our data demonstrate that more than $90 \%$ of the down regulated genes during acute hypoxia are repressed as a consequence of translational control.

Another interesting result of this study is that selective translation (stimulation or repression) is highly dynamic over time. Almost $80 \%$ of the translational induced genes demonstrate a 2 -fold increased translation efficiency during acute hypoxia. This percentage of genes reaches a minimum after 8 hours and then goes up and stabilizes at $44 \%$. A similar effect is observed for the repressed genes where after 2 hours of hypoxia nearly all of the translationally regulated genes exhibit over 2 -fold reduction in translation efficiency. This translational repression is somewhat suppressed after 8 hrs but becomes dominant again after prolonged hypoxic exposure. This kinetic picture of translational control extends earlier results from our lab from a single time point ${ }^{14}$ and presumably are reflective of the difference in regulation of eIF $2 \alpha$ and eIF4F at these different time points. Preferentially translated mRNAs during acute hypoxia are less dependent on eIF $2 \alpha$ availability while efficiently translated mRNAs after longer periods of hypoxia are less dependent on eIF4F. We cannot segregate the effects of these pathways in the current study, apart from their known kinetics of induction. As a result we are unable to clearly designate the ability for preferential translation of certain genes to either of these particular pathways. This 


\section{Chapter 5}

would require similar profiling of transcriptional and translational changes during hypoxia in cell lines defective in either pathway and these experiments are underway.

Transcripts that are less dependent on eIF $2 \alpha$ availability have been shown to harbor UORFs within their 5'UTR. Genes, like ATF-4 are translationally silenced under normal conditions by these RNA elements. Initiation of translation at UORFs occurs efficiently under normal conditions and can prevent translation of the actual ATF-4 ORF. However under conditions of ER stress eIF $2 \alpha$ is phosphorylated and the translation efficiency of these decoy uORFS is impaired. Subsequently, this allows the ribosomes to scan along the mRNA, bypass the decoy UORFs and initiate translation at the proper ATF-4 start codon ${ }^{22}$. Separate mechanisms confer translational specificity to manuscripts under conditions that affect the translation initiation complex eIF4F. Some mRNAs can utilize secondary structures within the $5^{\prime}$ UTR that act as internal ribosome entry sites to facilitate direct recruitment of the ribosomes to the transcript. Such transcripts are thus less dependent on initiation at the $5^{\prime}$ cap structure on the mRNA and thus also independent of the 5 'cap binding complex eIF4F. Since prolonged exposure to hypoxia induces disruption of eIF4F, this seems an ideal mechanism to maintain translation of important mRNA species. Several reports have shown that translation of HIF-1 $\alpha$, VEGF and BiP is mediated through this mechanism during hypoxia ${ }^{19-21}$, although this remains controversial for HIF- $1 \alpha^{36,37}$. Changes in gene expression that arise through regulation of translation are also expected to show a strong dependency on oxygen concentration. Several publications have shown that during more moderate hypoxia $(0.2-1.5 \%$ $\mathrm{O}_{2}$ ) phosphorylation of eIF $2 \alpha$ is substantially delayed ${ }^{10,13,27}$. It is very likely that this will affect gene expression differently than during anoxic conditions. Therefore it will be of interest to extend this study to other oxygen concentrations $\left(0.2-5 \% \mathrm{O}_{2}\right)$. This has recently been done using PC-3 human prostate cancer cells after a single exposure to $1.0 \% \mathrm{O}_{2}$ for 20 hours $^{27}$. In this study, Thomas and Johannes identified 104 induced 


\section{Microarray amalysis of translaced mRNA during hypoxha}

genes and 56 repressed genes showing more than 2-fold change in the polysomal fraction. Of these, only 33 and 31 genes of the induced and repressed genes respectively, demonstrated changes in translation efficiency. This number of translationally regulated genes is significantly smaller than that we observed in this current study and suggests that the severe hypoxia has a much stronger effect on translational control then moderate hypoxia. Nevertheless, to study this effect in more detail it will be important to utilize the same cell line throughout these studies as there might be differences in the dependence of different cell lines on the pathways that regulate translational control during hypoxia.

Translational control also appears to play an important role during tumorigenesis ${ }^{33,38-40}$. Mutations in genes that code for proteins involved in signaling to mTOR are frequently observed in cancer. This includes mutations in membrane kinases receptors like HER2/neu ${ }^{41}$ or in tumor suppressor genes like TSC1, TSC2 $2^{42,43}$ and LBK1 $1^{44}$. In addition several studies have clearly linked the mTOR regulated gene eIF4E directly to cancer development. Over expression of eIF4E causes transformation of immortalized 373 fibroblasts $^{38,45}$ and human mammary epithelial cells ${ }^{46}$. Knock down of eIF4E reduces cell proliferation and reverts the transformed phenotype ${ }^{47-49}$. Furthermore, transgenic mice over expressing eIF4E showed a significant increase in tumor development ${ }^{50,51}$. The effect of eIF4E on tumorigenesis has been attributed to its ability to decrease apoptosis in at least some tumor models ${ }^{51}$. Recently 2 reports performed polysomal gene profiling following overexpression of eIF4E ${ }^{39}$, 52 . Interestingly, Mamane et al. observed enhanced translation of transcripts encoding anti-apoptotic proteins ${ }^{39}$. Our study extends these results by demonstrating that translational control and its effects on gene expression are also highly dynamic and strongly influenced by hypoxic stress in tumors.

We performed gene ontology analysis on the selectively repressed and induced translated genes during acute and prolonged hypoxia in order to provide more insight into the mechanisms by which translational control 
affect hypoxia tolerance. Hypoxia down regulates translation rates of genes involved in protein metabolism, including transiation initiation factors, heat shock proteins and ribosomal proteins. These results seem consistent with mTOR inhibition that occurs during hypoxia as mTOR mediates increased mRNA translation of these genes.3. In addition, synthesis rates of proteins involved in cell growth and cell cycle are decreased and may contribute to the well known ability of hypoxia to decrease cell proliferation. Our data suggest that hypoxic cells utlize translation control to shut down these pro-proliferative processes. This might lead to a more persistent repression of mRNA translation compared to the rapid and reversible inhibition we observe during the first 24 hours of hypoxia. By decreasing the energy demand on the cell this could serve as an important survival mechanism to tolerate long term hypoxic stress.

The genes that we identified as being preferential translated during hypoxia encode for proteins involved in metabolism, signal transduction and transcription. Although these processes are clearly important in tumor cells, it remains to be seen how important these individual proteins are for hypoxia tolerance. Nevertheless the finding that many of the translationally enhanced genes encode for proteins involved in regulating transcription is interesting. This includes direct effects of translation on mRNA transcripts encoding transcription factors like MXI1 and CITED2 as well as transcriptional co regulators. These factors can antagonize c-myc and HIF transcriptional activity respectively and might therefore play a role during tumor development and the hypoxic response. We previously identified and characterized CITED2 as a preferentially regulated gene during hypoxia ${ }^{14,54}$.

In summary, we have shown that translational control contributes significantly to hypoxia regulated gene expression on a level that is comparable to transcriptional regulation. The magnitude of this effect suggests that translational control may be an interesting therapeutic target to pursue for hypoxia directed therapies. 


\section{Microarray analysis of translated mRNA during hyposia}

\section{References}

1. Brown JM, Wilson WR: Exploiting tumour hypoxia in cancer treatment, Nat Rev Cancer 2004, 4:437-447

2. Hockel M, Schlenger K, Aral B, Mitze M, Schaffer U, Vaupel P: Association between tumor hypoxia and malignant progression in advanced cancer of the uterine cervix, Cancer Res 1996, 56:4509-4515

3. Teicher BA: Physiologic mechanisms of therapeutic resistance. Blood flow and hypoxia, Hematol Oncol Clin North Am 1995, 9:475-506

4. Hockel M, Vaupel P: Biological consequences of tumor hypoxia, Semin Oncol 2001, 28:36-41.

5. Wouters BG, van den Beucken T, Magagnin MG, Koritzinsky M, Fels D, Koumenis $C$ : Control of the hypoxic response through regulation of $m R N A$ translation, Semin Cell Dev Biol 2005, 16:487-501

6. Wouters BG, van den Beucken T, Magagnin MG, Lambin P, Koumenis C: Targeting hypoxia tolerance in cancer, Drug Resist Updat 2004, 7:25-40

7. Semenza GL: Targeting HIF-1 for cancer therapy, Nat Rev Cancer 2003, $3: 721-732$

8. Schofield CJ, Ratcliffe PJ: Oxygen sensing by HIF hydroxylases, Nat Rev Mol Cell Biol 2004, 5:343-354

9. Koritzinsky $M$, Magagnin $M G$, van den Beucken $T$, Seigneuric $R$, Savelkouls K, Dostie J, Pyronnet S, Kaufman RJ, Weppler SA, Voncken JW, Lambin P, Koumenis C, Sonenberg N, Wouters BG: Gene expression during acute and prolonged hypoxia is regulated by distinct mechanisms of translational control, Embo J 2006, 25:1114-1125

10. Liu L, Cash TP, Jones RG, Keith B, Thompson CB, Simon MC: Hypoxiainduced energy stress regulates mRNA translation and cell growth, Mol Cell 2006, 21:521-531

11. Arsham AM, Howell JJ, Simon MC: A Novel Hypoxia-inducible Factorindependent Hypoxic Response Regulating Mammalian Target of Rapamycin and Its Targets, J. Biol. Chem. 2003, 278:29655-29660

12. Koumenis $C_{r}$ Naczki $C$, Koritzinsky M, Rastani S, Diehl A, Sonenberg $N$, Koromilas A, Wouters BG: Regulation of protein synthesis by hypoxia via activation of the endoplasmic reticulum kinase PERK and phosphorylation of the translation initiation factor eIF2alpha, Mol Cell Biol 2002, 22:74057416

13. Connolly E, Braunstein S, Formenti S, Schneider RJ: Hypoxia inhibits protein synthesis through a 4E-BP1 and elongation factor 2 kinase pathway controlled by mTOR and uncoupled in breast cancer cells, Mol Cell Biol 2006, 26:3955-3965

14. Koritzinsky $M$, Seigneuric $R$, Magagnin MG, van den Beucken $T$, Lambin $P$, Wouters BG: The hypoxic proteome is influenced by gene-specific changes in mRNA translation, Radiother Oncol 2005, 76:177-186

15. Bennewith $\mathrm{KL}$, Durand RE: Quantifying transient hypoxia in human tumor xenografts by flow cytometry, Cancer Res 2004, 64:6183-6189

16. Cardenas-Navia LI, Yu D, Braun RD, Brizel DM, Secomb TW, Dewhirst MW: Tumor-dependent Kinetics of Partial Pressure of Oxygen Fluctuations during Air and Oxygen Breathing, Cancer Res 2004, 64:6010-6017

17. van den Beucken T, Koritzinsky M, Wouters BG: Translational control of gene expression during hypoxia, Cancer Biol Ther 2006, 5:749-755

18. Holcik $M$, Sonenberg $N$, Korneluk RG: Internal ribosome initiation of translation and the control of cell death, Trends Genet 2000, 16:469-473 
19. Lang KJ, Kappel A, Goodall G]: Hypoxia-inducible factor-1alpha mRNA contains an internal ribosome entry site that allows efficient translation during normoxia and hypoxia, Mol Biol Cell 2002, 13:1792-1801

20. Stein I, Itin A, Einat P, Skaliter R, Grossman Z, Keshet E: Translation of vascular endothelial growth factor mRNA by internal ribosome entry: implications for translation under hypoxia, Mol Cell Biol 1998, 18:31123119

21. Thoma C, Bergamini G, Galy B, Hundsdoerfer P, Hentze MW: Enhancement of IRES-mediated translation of the c-myc and BiP mRNAs by the poly $(A)$ tail is independent of intact eIF4G and PABP, Mol Cell 2004, 15:925-935

22. Harding HP, Novoa I, Zhang $Y$, Zeng $H$, Wek $R$, Schapira $M$, Ron D: Regulated translation initiation controls stress-induced gene expression in mammalian cells, Mol Cell 2000, 6:1099-1108

23. Bartel DP: MicroRNAs: genomics, biogenesis, mechanism, and function, Cell 2004, 116:281-297

24. Kulshreshtha R, Davuluri RV, Calin GA, Ivan M: A microRNA component of the hypoxic response, Cell Death Differ 2008, 15:667-671

25. Kuishreshtha $R$, Ferracin $M$, Negrini $M$, Calin GA, Davuluri RV, Ivan $M$ : Regulation of microRNA expression: the hypoxic component, Cell Cycle 2007, 6:1426-1431

26. Vasudevan S, Tong $Y$, Steitz JA: Switching from repression to activation: microRNAs can up-regulate translation, Science 2007, 318:1931-1934

27. Thomas JD, Johannes GJ: Identification of mRNAs that continue to associate with polysomes during hypoxia, Rna 2007, 13:1116-1131

28. Blais JD, Filipenko V, Bi M, Harding HP, Ron D, Koumenis C, Wouters BG, Bell JC: Activating transcription factor 4 is translationally regulated by hypoxic stress, Mol Cell Biol 2004, 24:7469-7482

29. Blais JD, Addison $C L$, Edge $R$, Falls $T$, Zhao $H$, Wary $K$, Koumenis $C$, Harding HP, Ron D, Holcik M, Bell JC: Perk-dependent translational regulation promotes tumor cell adaptation and angiogenesis in response to hypoxic stress., Mol. Cell. Biol. 2006, 26:9517-9532

30. $\mathrm{Ma} Y$, Hendershot LM: Delineation of a negative feedback regulatory loop that controls protein translation during endoplasmic reticulum stress, ] Biol Chem 2003, 278:34864-34873

31. Pause A, Belsham GJ, Gingras AC, Donze O, Lin TA, Lawrence JC, Jr., Sonenberg $\mathrm{N}$ : Insulin-dependent stimulation of protein synthesis by phosphorylation of a regulator of $5^{\prime}$-cap function, Nature 1994, 371:762767

32. Hosack DA, Dennis G, Jr., Sherman BT, Lane HC, Lempicki RA: Identifying biological themes within lists of genes with EASE, Genome Biol 2003, 4:R70

33. Rajasekhar VK, Viale A, Socci ND, Wiedmann M, Hu X, Holland EC: Oncogenic Ras and Akt signaling contribute to glioblastoma formation by differential recruitment of existing mRNAs to polysomes, Mol Cell 2003, 12:889-901

34. Lu X, de la Pena L, Barker C, Camphausen K, Tofilon PJ: Radiation-induced changes in gene expression involve recruitment of existing messenger RNAs to and away from polysomes, Cancer Res 2006, 66:1052-1061

35. Hochachka PW, Buck LT, Doll CJ, Land SC: Unifying theory of hypoxia tolerance: molecular/metabolic defense and rescue mechanisms for surviving oxygen lack, Proc Natl Acad Sci U S A 1996, 93:9493-9498

36. Bert $A G$, Grepin $R$, Vadas MA, Goodall GJ: Assessing IRES activity in the HIF-1 \{alpha\} and other cellular 5' UTRs, Rna 2006, 12:1074-1083 
37. Young RM, Wang SJ, Gordan JD, Ji X, Liebhaber SA, Simon MC: Hypoxiamediated selective mRNA translation by an internal ribosome entry site independent mechanism, ] Biol Chem 2008,

38. Zimmer SG, DeBenedetti A, Graff JR: Translational control of malignancy: the mRNA cap-binding protein, eIF- $4 E$, as a central regulator of tumor formation, growth, invasion and metastasis, Anticancer Res 2000, 20:1343-1351

39. Mamane $Y$, Petroulakis E, Martineau $Y$, Sato TA, Larsson O, Rajasekhar VK, Sonenberg N: Epigenetic activation of a subset of mRNAs by eIF4E explains its effects on cell proliferation, PLoS ONE 2007, 2:e242

40. Holland EC, Sonenberg N, Pandolfi PP, Thomas G: Signaling control of mRNA translation in cancer pathogenesis, Oncogene 2004, 23:3138-3144

41. Laughner E, Taghavi P, Chiles K, Mahon PC, Semenza GL: HER2 (neu) signaling increases the rate of hypoxia-inducible factor lalpha (HIF1alpha) synthesis: novel mechanism for HIF-1-mediated vascular endothelial growth factor expression, Mol Cell Biol 2001, 21:3995-4004

42. Young J, Povey S: The genetic basis of tuberous sclerosis, Mol Med Today 1998, 4:313-319

43. Kwiatkowski DJ: Tuberous sclerosis: from tubers to mTOR, Ann Hum Genet 2003, 67:87-96

44. Boudeau J, Sapkota G, Alessi DR: LKB1, a protein kinase regulating cell proliferation and polarity, FEBS Lett 2003, 546:159-165

45. Lazaris-Karatzas A, Montine KS, Sonenberg N: Malignant transformation by a eukaryotic initiation factor subunit that binds to mRNA $5^{\prime}$ cap, Nature 1990, 345:544-547

46. Avdulov $S$, Li S, Michalek V, Burrichter D, Peterson M, Perlman DM, Manivel JC, Sonenberg N, Yee D, Bitterman PB, Polunovsky VA: Activation of translation complex eIF4F is essential for the genesis and maintenance of the malignant phenotype in human mammary epithelial cells, Cancer Cell 2004, 5:553-563

47. Oridate N, Kim HJ, Xu X, Lotan R: Growth inhibition of head and neck squamous carcinoma cells by small interfering RNAs targeting eIF4E or cyclin D1 alone or combined with cisplatin, Cancer Biol Ther 2005, 4:318323

48. De Benedetti A, Joshi-Barve S, Rinker-Schaeffer C, Rhoads RE: Expression of antisense RNA against initiation factor eIF-4E mRNA in HeLa cells results in lengthened cell division times, diminished translation rates, and reduced levels of both eIF-4E and the p220 component of eIF-4F, Mol Cell Biol $1991,11: 5435-5445$

49. Rinker-Schaeffer CW, Graff JR, De Benedetti A, Zimmer SG, Rhoads RE: Decreasing the level of translation initiation factor 4E with antisense RNA causes reversal of ras-mediated transformation and tumorigenesis of cloned rat embryo fibroblasts, Int J Cancer 1993, 55:841-847

50. Ruggero D, Montanaro L, Ma L, XU W, Londei P, Cordon-Cardo C, Pandolfi PP: The translation factor eIF-4E promotes tumor formation and cooperates with c-Myc in lymphomagenesis, Nat Med 2004, 10:484-486

51. Wendel HG, De Stanchina E, Fridman JS, Malina A, Ray S, Kogan S, Cordon-Cardo C, Pelletier J, Lowe SW: Survival signalling by Akt and eIF4E in oncogenesis and cancer therapy, Nature 2004, 428:332-337

52. Larsson O, Li S, Issaenko OA, Avdulov S, Peterson M, Smith K, Bitterman $\mathrm{PB}$, Polunovsky VA: Eukaryotic translation initiation factor $4 \mathrm{E}$ induced progression of primary human mammary epithelial cells along the cancer 


\section{Chapter 5}

pathway is associated with targeted translational deregulation of oncogenic drivers and inhibitors, Cancer Res 2007, 67:6814-6824

53. Meyuhas O HE: Translational control of TOP mRNAs. Edited by Sonenberg, $\mathrm{N} \mathrm{HJ}$, Mathews MB. 2000, 671-694 p

54. van den Beucken $T$, Magagnin $M G$, Savelkouls $K$, Lambin $P$, Koritzinsky $M$, Wouters BG: Regulation of Cited 2 expression provides a functional link between translational and transcriptional responses during hypoxia, Radiother Oncol 2007, 83:346-352 


\section{CHAPTER 6}

Regulation of Cited 2 expression provides a functional link between translational and transcriptional responses düring hypoxia

Radiother Oncol. 2007 Jun; 83(3):346-52.

van den Beucken T, Magagnin MG, Savelkouls K, Lambin P, . Koritzinsky $M$, Wouters $B G$. 


\section{Abstract}

Background and purpose: Protein synthesis rates are greatly reduced under hypoxic conditions as a consequence of an overall inhibition of mRNA translation. Certain specific MRNA species have the ability to escape this general translational repression. At the cellular level this results in differential protein expression during hypoxic conditions. The objective of this study was to characterize the translational regulation of the postulated HIF-1u antagonist Cited2.

Materials and methods: DU145 prostate carcinoma cells and mouse embryonic fibroblasts with a homozygous knock-in mutation for elf2u. (S51A) or wild-type eIF $2 *$ were exposed to severe hypoxia after which both total MRNA and efficiently translated mRNA were isolated. Quantitative RT-PCR was used to measure and compare changes in transcription (total mRNA) with changes in translation (efficiently translated mRNA fraction).

Results: We show using HIF-1 1 null MEF cells that transcriptional induction of Cited 2 during hypoxia is dependent on HIF-1 1 . Although global mRNA translation is inhibited during hypoxia Cited 2 mRNA remains efficiently translated. An evolutionary conserved upstream open reading frame (UORF) in the 5'UTR of Cited 2 did not stimulate translation in an eIF $2 \alpha$ dependent manner during hypoxia.

Conclusions: Selective translation Cited 2 by an eIF $2 \alpha$ independent mechanism establishes a link between translation and HIF-1 dependent transcription during hypoxia. 


\section{Introduction}

Tumor cells are frequently exposed to conditions of stress including nutrient starvation, oxidative stress, and hypoxia. Cellular adaptation to these conditions facilitates both cell survival and overall tumor growth. For tumor hypoxia, these adaptive mechanisms also appear to contribute to increased malignancy and poor prognosis by promoting a more aggressive tumor phenotyp $\mathrm{e}^{1-4}$. The best understood adaptive response to hypoxia occurs through activation of hypoxia inducible transcription factor 1 (HIF1). This transcription factor plays a pivotal role in the cellular response to hypoxia by inducing transcription of more than 60 down stream genes ${ }^{5}$. HIF- 1 is a heterodimer consisting of $\alpha$ and $\beta$ subunits that are both ubiquitously expressed. However, under aerobic conditions HIF-1 $\alpha$ has an extremely short half-life due to its efficient ubiquitination by the von Hippel-Lindau tumor suppressor protein ( $\mathrm{pVHL}$ ) and subsequent degradation ${ }^{6}$. The ability of $\mathrm{pVHL}$ to recognize HIF-1 $\alpha$ is dependent on hydroxylation of proline residues 402 and 564 in HIF-1 $\alpha$ which is carried out by a family of oxygen-sensitive prolyl hydroxylases (PHD1-3). Under hypoxic conditions hydroxylation cannot occur, resulting in HIF- $1 \alpha$ stabilization and formation of a functional transcription factor with HIF-1 $\alpha$. This complex mediates transcription by binding to hypoxia responsive elements (HREs) within HIF responsive genes. In addition to regulation by protein stability, hypoxia also influences the transcriptional activity of HIF1. Efficient transcriptional activity requires interaction of the carboxyterminal transactivation domain (CTAD) in HIF-1 $\alpha$ with the transcriptional co-activator $\mathrm{CBP} / \mathrm{p} 300^{7}, 8$. Under aerobic conditions, this interaction is suppressed by hydroxylation of asparagine residue 803 in the CTAD by another oxygen sensitive enzyme called $\mathrm{FIH}^{9,10}$.

A second important mechanism for regulating gene expression during hypoxia is through control of mRNA translation ${ }^{11}$. Translational control provides a rapid and reversible manner of regulating gene expression, in contrast to transcriptional effects that can take hours to occur. Therefore, regulating gene expression by translation might be especially important in 


\section{Chapter 6}

tumor cells that experience transient periods of hypoxia that are too short to elicit a significant transcriptional response. Such transient fluctuations in oxygen supply are well documented ${ }^{12}{ }^{13}$ ranging from severe hypoxia (0\%) to $\sim 4 \% \mathrm{O}_{2}$ and are the consequence of the irregular and immature tumor vasculature. Regulation of mRNA translation has emerged as an important contributor to differential gene expression not only during hypoxia ${ }^{14,} 15$ but also after irradiation ${ }^{16}$ or oncogenic signaling ${ }^{17}$. Work from our group and others has shown that global initiation of mRNA translation is rapidly inhibited upon exposure to hypoxia ${ }^{18,}{ }^{19}$. This inhibition is mediated by at least two independent pathways that display different activation kinetics. A rapid, but transient, response is elicited through activation of the endoplasmic reticulum kinase PERK and subsequent phosphorylation of eukaryotic initiation factor $2 \alpha(\text { eIF } 2 \alpha)^{20}$. This pathway dictates the early response to hypoxia. During chronic hypoxia, data suggest that disruption of eukaryotic initiation factor $4 \mathrm{~F}$ (eIF4F) complex and sequestration of eIF4E by both its inhibitor 4E-BP1, and its transporter $4 \mathrm{E}-\mathrm{T}$ maintain inhibition of translation. Although both of these pathways lead to global inhibition in translation, individual mRNA transcripts can be affected to highly variable degrees ${ }^{18}$, ultimately resulting in hypoxic specific changes is protein expression.

In order to identify translationally controlled genes we previously performed a gene profiling study on the subset of efficiently translated mRNAs in DU145 prostate carcinoma cells after exposure to hypoxia ${ }^{15}$. We identified the transcriptional activator Cited2 as one of the genes whose translation was significantly increased during hypoxia. Cited 2 has also been shown to be regulated at the transcriptional level during hypoxia ${ }^{21}$. Although it has not been definitively demonstrated, this induction is likely due to HIF-1, since Cited 2 has been reported to contain 3 putative HREs that HIF-1 can bind to ${ }^{21}$. Interestingly, Cited 2 can compete with HIF-1 for binding the coactivator $\mathrm{CBP} / \mathrm{p} 300$ through its $\mathrm{CH} 1$ domain ${ }^{22}$. Therefore, by sequestration of $\mathrm{CBP} / \mathrm{p} 300$, Cited 2 is able to act as a negative feedback loop that limits the transcriptional activity of HIF-1 and thus HIF-1 
responsive gene activation ${ }^{23}$. Here we have investigated in detail the transcriptional and translational regulation of Cited2 to explore a possible link between hypoxic control of mRNA translation and HIF activity during hypoxia.

\section{Materials and Methods}

\section{Tissue culture conditions}

Exponentially growing prostate carcinoma cells (DU145), mouse embryonic fibroblasts (MEFs) wild type, HIF-1 $\alpha$ null or that had a homozygous knock-in mutation for eIF $2 \alpha$ (S51A) were grown on glass dishes in McCoy and DMEM media respectively supplemented with $10 \%$ foetal calf serum (FCS) (Sigma-Aldrich). For hypoxic exposure cells were transferred into a MACS VA500 microaerophilic workstation (Don Whitley Scientific, Shipley, UK). The atmosphere in the chamber consisted of $5 \%$ $\mathrm{H}_{2}, 5 \% \mathrm{CO}_{2}, 0 \% \mathrm{O}_{2}$, and $90 \% \mathrm{~N}_{2}$.

\section{Isolation of polysomal RNA}

Polysomal fractionation was performed as described previously ${ }^{18}$. Briefly, after treatment with cycloheximide $(\mathrm{CHX})$ for $3 \mathrm{~min}$ at $37^{\circ} \mathrm{C}$ cells were washed twice with ice-cold PBS/CHX, scraped and lysed in $1 \%$ Triton X100 and 100 units RNAse-In (Ambion) in Buffer $\mathrm{A}(0.3 \mathrm{M} \mathrm{NaCl}, 15 \mathrm{mM}$ $\mathrm{MgCl} 2,15 \mathrm{mM}$ Tris ( $\mathrm{pH} 7.4), 0.1 \mathrm{mg} / \mathrm{ml} \mathrm{CHX}$ ) at $4^{\circ} \mathrm{C}$. Nuclei were removed, $200 \mathrm{mg} / \mathrm{ml}$ heparin was added and residual debris was removed by centrifugation. The lysate was layered on a $10 \mathrm{ml}$ continuous sucrose gradient $(20-50 \%$ sucrose and $0.1 \mathrm{mg} / \mathrm{ml}$ heparin in Buffer $\mathrm{A}$ ). After 90 min of centrifugation at $39,000 \mathrm{rpm}$ in an SW41-Ti rotor at $4{ }^{\circ} \mathrm{C}$, the absorbance at $254 \mathrm{~nm}$ was measured continuously as a function of gradient depth in a BioRad Laboratories UV monitor and fractions were collected in $0.55 \mathrm{M}$ final concentration of guanidinium- $\mathrm{HCl}$. RNA isolation was performed as previously described ${ }^{18}$. 


\section{Quantitative Real-time PCR}

Real-time PCR was performed in ABI 7500 (Applied Biosystems). RNA samples were reverse transcribed using $M M L$ reverse transcriptase (Sigma-Aldrich) and random decamers (Ambion). The abundance of the following genes were detected with Taqman $^{\circledR}$ Gene Epression Arrays (Applied Biosystems); human cited2 Hs00366696-m1, mouse actin: Mm00607939_s1, mouse ATF4: mm00515324_m1, mouse CA9: mm00519870_m1, mouse cited2 Mm00516121_m1 and 18SrRNA: Hs99999901_s1. Following genes were measured with SYBR ${ }^{\circledR}$ Green I (Applied Biosystems) using the indicated forward and reverse primers: human $\beta$-Actin: F-accatggatgatgatatcgcc and R-gccttgcacatgccgg; human ATF-4: F-tggccaagcacttcaaacct and R-gttgttggagggactgaccaa. Total mRNA samples were normalized by $18 \mathrm{~S}$ rRNA. Samples from polysomal fractions were normalized by $18 \mathrm{~S}$ rRNA measured by PCR divided by $18 \mathrm{~S}$ rRNA measured by spectrometry during fractionation. This facilitates correction for any differences in RNA isolation or reverse transcription efficiency between samples. Error bars represent the standard error of the mean from 3 independent experiments.

\section{Results}

As discussed above, Cited 2 has been shown to be transcriptionally regulated during hypoxia. Since it contains a putative HRE, we first investigated whether transcriptional induction was strictly dependent on HIF-1. To this end, we measured transcriptional induction of Cited2 in HIF$1 \alpha$ WT and HIF-1 $\alpha$ null MEFs. As can be seen in Fig.1, transient upregulation of Cited 2 is limited to HIF- $1 \alpha$ proficient MEF cells implying that Cited2 is indeed regulated by HIF-1 during hypoxia. Several other known HIF target genes including VEGF and CA9 were also exclusively induced in WT HIF-1 $\alpha$ MEFs (data not shown). These data suggest that HIF-1 may indeed limit its own transcriptional activity via upregulation of Cited2. 
In order to study the effect of hypoxia on Cited2 mRNA translation we isolated efficiently translated polysomal mRNA (associated with five or more ribosomes) from DU145 cells using sucrose gradients. Two

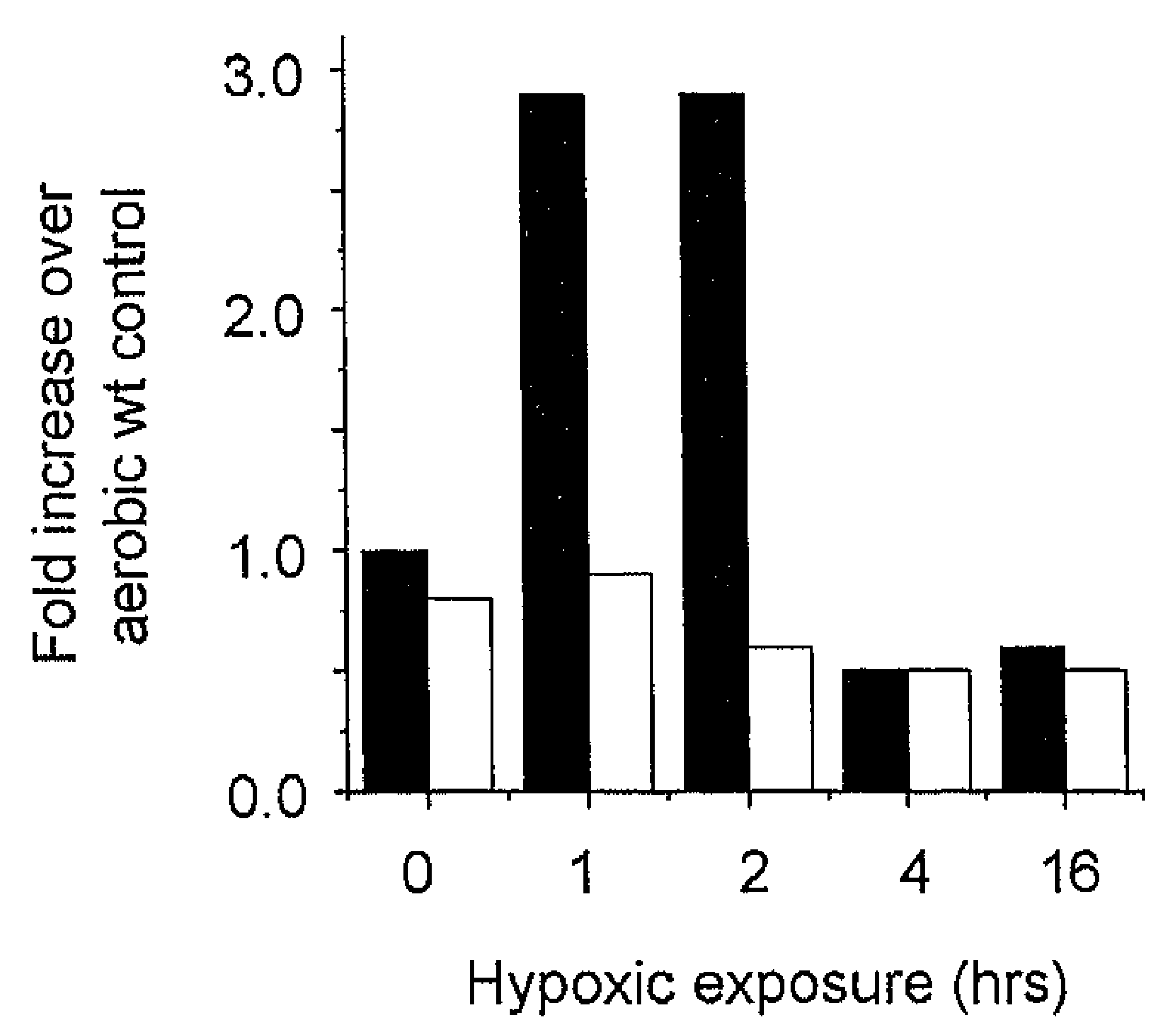

Figure 1. HIF-1 dependent induction of Cited2 mRNA upon hypoxia. Cited 2 mRNA levels were determined using quantitative real-time PCR in wild type (black bars) and HIF null (white bars) MEF cells. Representative result of 2 independent experiments is shown.

representative polysome profiles from aerobic and hypoxic $(2 h)$ conditions are shown in fig. 2A. The lowest molecular weight peaks represent the $40 S$ and 605 ribosomal subunits respectively. The following peaks represent mRNAs with increasing numbers of associated ribosomes. From these profiles the percentage of ribosomes that are participating in translation can be obtained by dividing the integrated area of the translated fraction by the total integrated area. This value gives an estimate of the amount of ongoing de novo protein synthesis. Under aerobic conditions approximately $54 \%$ of the ribosomes reside in the highly translated fraction, whereas this drops to only $22 \%$ after 2 hours of hypoxia. Translation in these cells remains inhibited for up to 24 hours (data not shown).

To assess the translation efficiency of Cited2 we measured Cited2 transcript levels in both the total mRNA fraction as well as in the highly translated polysomal fraction. Fig. $2 \mathrm{~B}$ shows that total cellular levels of Cited 2 mRNA rise rapidly ( $>3$ fold within 2 hours) and continuously over 


\section{Chapter 6}

A
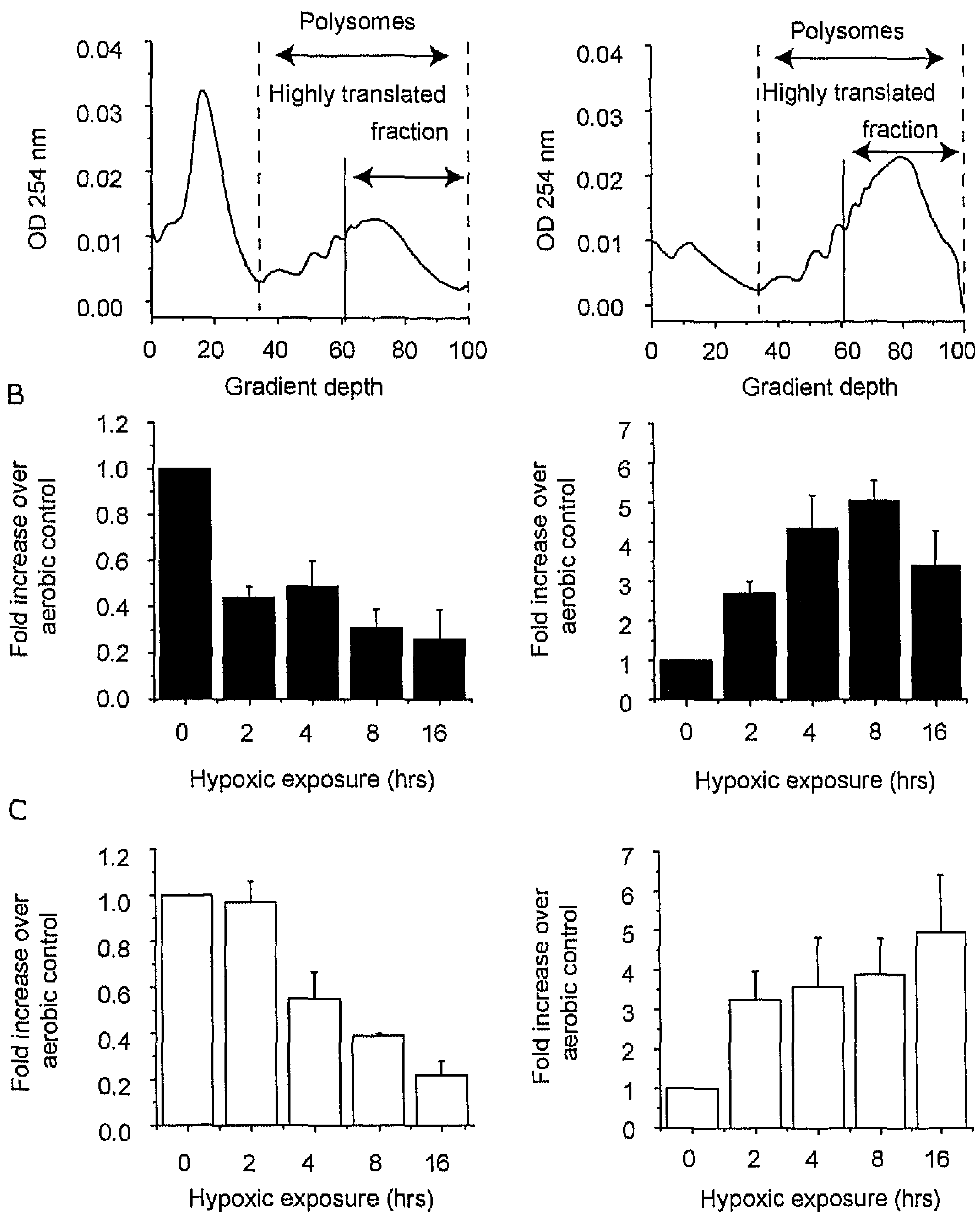

Figure 2. Cited2 mRNA transcripts are efficiently translated during hypoxia. DU145 cells were exposed to $0 \%$ hypoxia for $0-16$ hrs and cell lysates were separated on sucrose gradients. (A) Typical optical density profile at $254 \mathrm{~nm}$ is shown as a function of gradient depth for aerobic exposure (left panel) and after 2 hrs of hypoxia (right panel). Total mRNA levels (white bars) and transcript abundance of efficiently translated mRNAs in polysomal fraction (black bars) were determined by quantitative RT-PCR for Cited2 (B) and $\beta$-actin (C) in DU145 cells. 


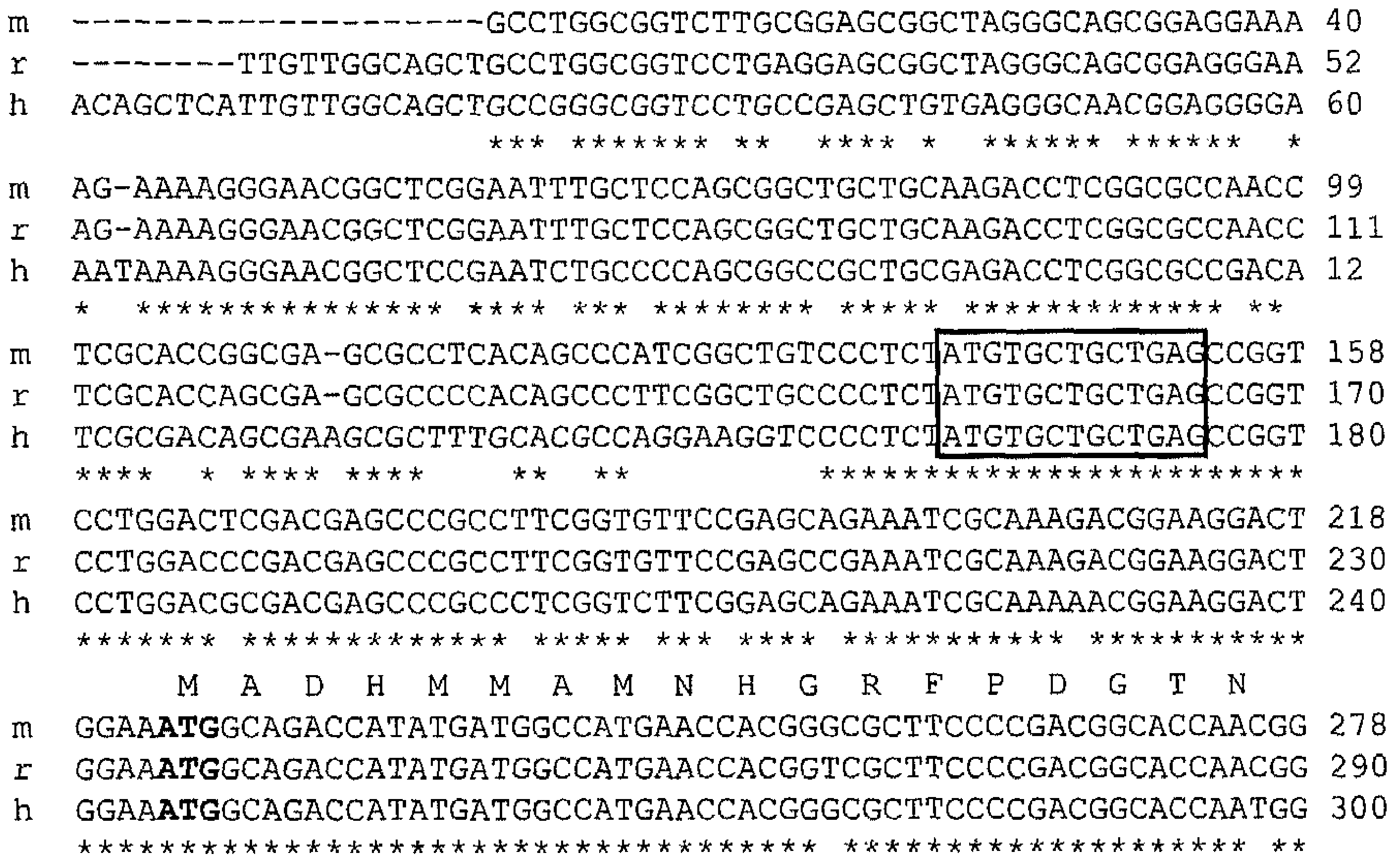

Figure 3. Evolutionary conserved UORF in 5'UTR of Cited2. Sequence alignment of human, mouse and rat $5^{\prime}$ untranslated regions of Cited2. GenBank ${ }^{T M}$ accession number respectively NM_006079, NM_010828 and NM_053698. Identical nucleotides are marked by an asterisk, the conserved uORF is marked by a box and ATG start codon for the Cited2 ORF is indicated in bold.

time for up to 16 hours of hypoxia. Interestingly, despite the overall mRNA found within the well-translated fraction over time is similar to the fold-increase in transcription. This demonstrates that Cited2 remains efficiently translated during hypoxia under conditions where overall mRNA suppression in mRNA translation (Fig. 2A), the fold-increase in Cited2translation is greatly reduced. In comparison, the amount of mRNA that remains efficiently translated for the housekeeping gene $\beta$-actin decreases to less than $40 \%$ after 2 hours of hypoxia. At this time point, no significant change in total cellular $\beta$-actin transcripts is observed. Thus, as expected, $\beta$-actin translation falls during hypoxia to levels that are similar to the overall drop in cellular translation.

Selective translation of specific transcripts under certain circumstances is often determined by sequences within the $5^{\prime}$ or $3^{\prime}$ untranslated regions 
(UTRs) of those transcripts. We reasoned that if such a sequence was present and important for regulation of Cited 2 it should be evolutionarily conserved. Interestingly sequence alignment of the 5'UTR from several different species revealed a high sequence similarity (Fig. 3). Furthermore, all species contained a conserved upstream open reading frame (UORF) within its $5^{\prime}$ UTR. The presence of UORFs has previously been implicated in the translational induction of other genes, including the hypoxia responsive gene activating transcription factor 4 (ATF4). Consistent with these previous findings, we found that hypoxia stimulated the translation of ATF4 at 2 and 4 hours of exposure even though total mRNA abundance remained unchanged (Fig. 4).

A

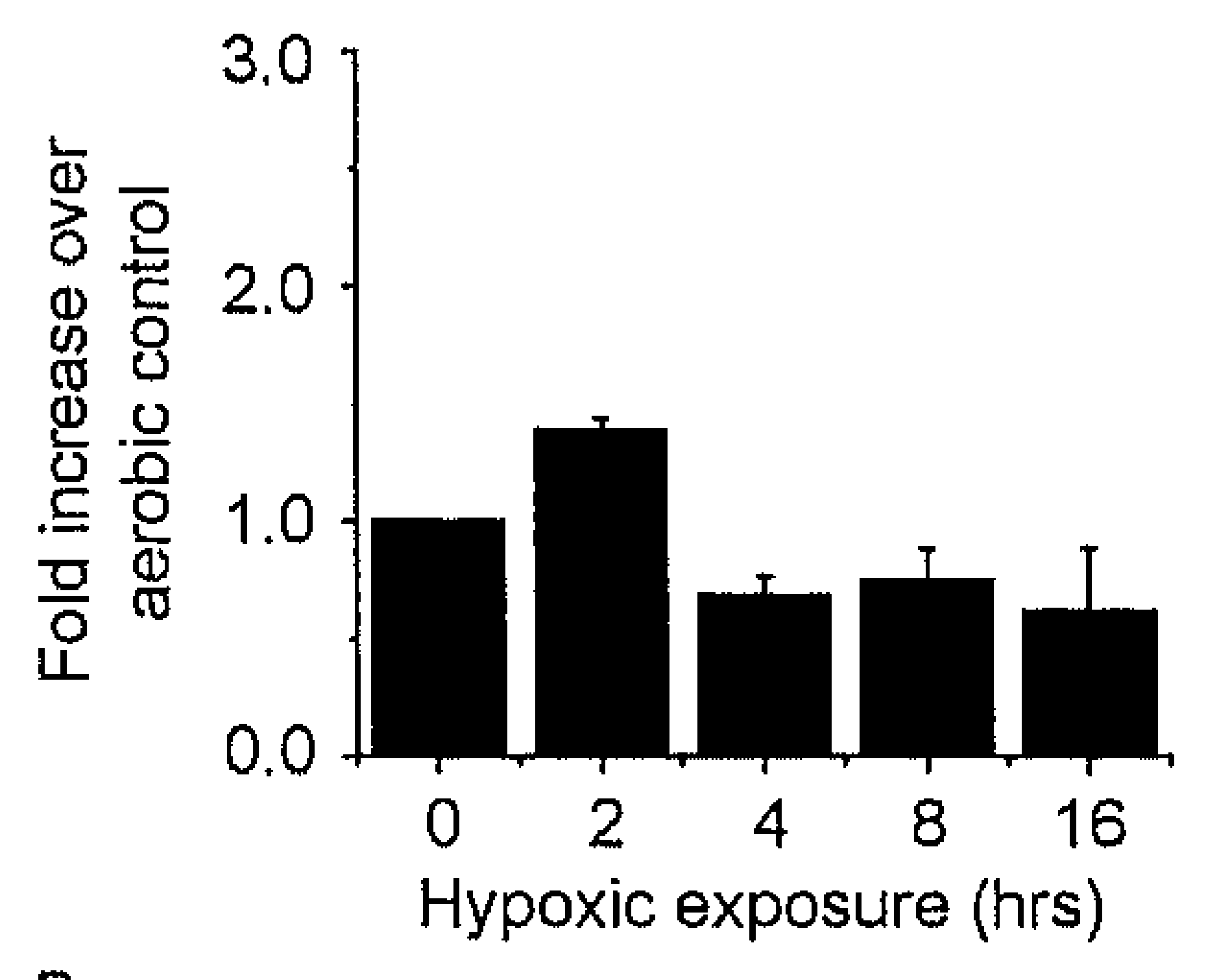

B

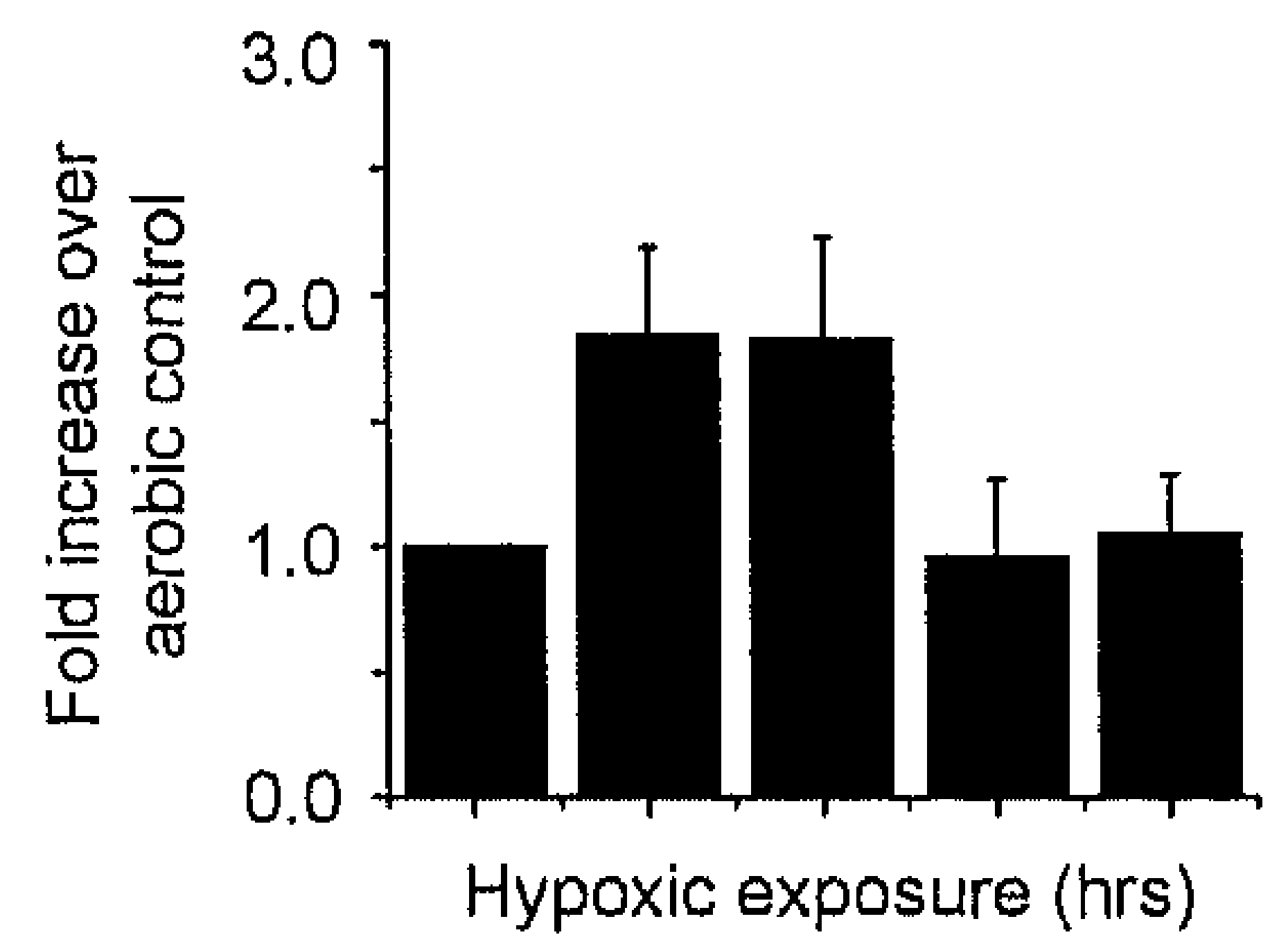

Figure 4. Translational control of ATF4 regulation. (A) ATF-4 mRNA abundance in DU145 cells was measured as function of time during hypoxia. (B) Presence of efficiently translated ATF-4 transcripts in polysomal fraction. Results from 3 independent experiments are shown with SEM.

Although both ATF4 and Cited 2 contain a UORF, the fact that the increase in translation of ATF4 is transient and occurs without a corresponding transcriptional induction suggests that their translational control may be 
distinct. To test this further, we assessed translational changes in ATF4 and Cited 2 in cells that have been shown to be defective in hypoxic regulation of ATF4 ${ }^{24}$. Since ATF4 induction is strictly dependent on eIF2 $\alpha$ phosphorylation we used MEF cells with a homozygous S51A knock-in mutation in eIF $2 \alpha$. This mutant eIF2 $\alpha$ allele cannot be phosphorylated upon hypoxia and thus the S51A cells are unable to inhibit overall mRNA translation during acute hypoxia ${ }^{18}$. We measured both total cellular and well-translated transcript levels after 4hours of hypoxia. In addition, we measured transcript levels following 1 hour of reoxygenation because at this time both eIF $2 \alpha$ phosphorylation and overall mRNA translation return to normal levels. Both Cited 2 and ATF4 showed a small increase ( $\sim 2$ fold) in total cellular mRNA after 4 hours of hypoxia and this did not differ between the WT and S51A cells (Fig.5A). In the WT cells, translation of

A
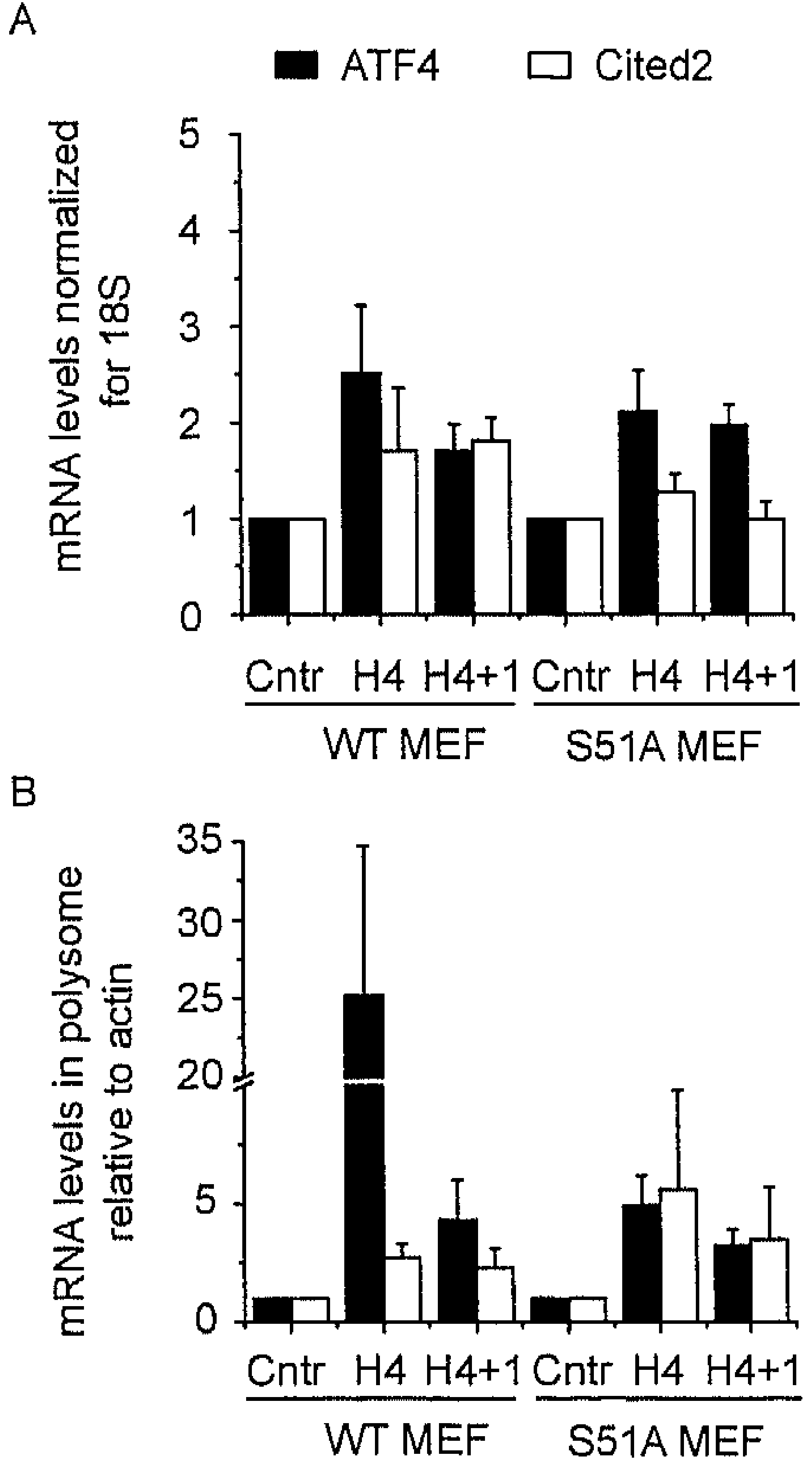

Figure 5. Translation control of Cited 2 is independent of eIF $2 \alpha$ phosphorylation. (A) Total MRNA levels in WT MEF and S51A MEF cells after acute hypoxia or hypoxia plus reoxygenation. (B) Accumulation of ATF4 and Cited2 mRNA transcripts in highly translated fraction relative to the housekeeping gene $\beta$-actin. Results from 3 independent experiments are shown with SEM. 


\section{Chapter 6}

ATF4 was greatly increased after 4 hours of hypoxia. Compared with $\beta$ actin, which behaves similarly to overall changes in translation, the amount of well-translated ATF4 increased by $\sim 25$ fold (Fig.5B). This increase was dependent on eIF2 $\alpha$ phosphorylation as it did not occur in the S51A cells and it returned to near normal levels after 1 hour of reoxygenation. In contrast, the amount of well-translated Cited 2 mRNA showed a comparatively much smaller increase after 4 hours. Furthermore, translation of Cited 2 mRNA was not impaired in S51A MEFs and may even be slightly increased, although this is not significant $(p>0.15)$. These data suggest that despite the presence of a conserved UORF, translational control of Cited 2 during hypoxia is independent of eIF2 $\alpha$ phosphorylation.

\section{Discussion}

The oxygen-sensing pathways present in tumor cells are important determinants of hypoxia tolerance, gene expression and cell phenotype. Several important pathways that influence protein expression during hypoxia have emerged, including a transcriptional response driven in large part by HIF-1. In addition, hypoxia causes a rapid and sustained inhibition in global translation that can also lead to selective gene expression due to the fact that mRNA transcripts from specific genes show differences in their ability to be translated during hypoxia ${ }^{18}$. This may account for the ability of cells to rapidly alter protein expression, as evidenced by detectable changes in the proteome within 1 hour of exposure to hypoxia $^{15}$. To date, the transcriptional and translational pathways appear to be largely distinct with little known influence on each other.

We recently identified Cited 2 as part of the subset of genes whose translation is induced during hypoxia ${ }^{15}$. Cited2 has been postulated to act as a negative feedback loop for HIF-1 transactivation by competing for the transcriptional co-activator $\mathrm{CBP} / \mathrm{p} 300^{21}, 23$. This feedback loop may be generated by HIF-1 itself, since both hypoxia and deferoxamine induce Cited 2 at the mRNA level ${ }^{21}$ and because the Cited 2 promoter contains 3 
consensus HREs ( $5^{\prime}$ RCGTG) that bind the HIF-1 heterodimer. However, definitive evidence that HIF-1 is required for induction has not, to our knowledge been demonstrated. Our data presented in figure 1 provide strong evidence that Cited 2 is indeed a bona fide HIF- $1 \alpha$ target since the transcriptional induction is completely abolished in HIF-1 $\alpha$ null cells.

Since Cited 2 acts as an inhibitor of HIF-1 transcriptional activity, its additional regulation by translation provides a potential link between translational changes during hypoxia and HIF-1 mediated transcriptional changes. We therefore explored in detail the transcriptional and translational control of Cited 2 during acute severe hypoxia. Our results show that Cited 2 is induced to similar levels in both transcription and translation and this occurs within 2 hours and continues to rise for up to 16 hours. (Fig.2). The ability for preferential translation during hypoxia is illustrated by the fact that the increase in translation closely follows the transcriptional induction. This while overall levels of mRNA translation are greatly decreased like we showed for $\beta$-actin which represents the average gene. It will be interesting to determine whether Cited2 is also preferentially translated during moderate hypoxia. Especially since our lab recently found that acute moderate hypoxia also causes inhibition of mRNA translation mediated by the same mechanism as during severe hypoxia ${ }^{25}$.

There still is limited knowledge regarding the mechanisms responsible for selective translation of different genes during cellular stress, including hypoxia. The translation efficiency of individual genes is thought to be regulated largely by the $5^{\prime}$ and $3^{\prime}$ untranslated parts of the mRNA. Sequence alignment of the 5'UTR of Cited 2 from different species revealed an evolutionary conserved uORF. Under normal growth conditions an uORF acts as a repressor of protein synthesis by engaging the ribosome at its start site and thereby preventing translation of the correct downstream ORF. However, somewhat paradoxically, translation of UORF containing transcripts can be stimulated under conditions where eIF2 $\alpha$ is phosphorylated and overall mRNA translation is inhibited. Phosphorylation 


\section{Chapter 6}

of eIF $2 \alpha$ reduces the rate at which initiation of translation can occur, and this is thought to allow time for the ribosome to scan through the 'decoy' UORF and reach the correct downstream ORF before beginning protein synthesis. This is the case for ATF4, whose transcript is more efficiently translated during hypoxia when eIF $2 \alpha$ is phosphorylated ${ }^{20,26}$. However, we found that although Cited 2 contains a conserved uORF, its preferential translation during hypoxia was not similarly dependent on phosphorylation of eIF $2 \alpha$.

It remains unclear if the conserved UORF in Cited 2 contributes to its selective translation during hypoxia, and there are other candidate elements in the 5'UTR that might be important. It has been reported that a number of hypoxia regulated genes including HIF-1 $\alpha$, VEGF and BiP contain an internal ribosome binding site (IRES) within their $5^{\prime}$ UTR ${ }^{27-29}$. An IRES consists of a secondary mRNA structure that facilitates direct ribosome binding to the mRNA independent of the eIF4F complex at the 5 cap structure. Since disruption of the eIF4F complex also occurs during hypoxia ${ }^{18}$, it is tempting to speculate that the Cited $25^{\prime}$ UTR may harbor an IRES that stimulates its translation during hypoxia. This possibility warrants further investigation.

Regardless of the exact mechanism, our data show that hypoxia induced changes in translation efficiency effect the expression of Cited2, and thus the transcriptional potency of HIF-1. This provides the first evidence of a link between the rapid activation of translation inhibition by hypoxia and the transcriptional response driven by HIF-1. Interestingly, a link from HIF-1 back to translation has also been reported. HIF-1 is responsible for induction of the REDD1 gene, which functions as an inhibitor of mTOR signaling ${ }^{30}$. Inhibition of MTOR has been reported to occur during hypoxia and may be responsible in part for decreased translation due to its ability to regulate cap-dependent translation ${ }^{31}$. Together with the data presented here, these results indicate that crosstalk between transcriptional and translational changes during hypoxia may be more ubiquitous than previously recognized. 


\section{Acknowledgements}

We wish to acknowledge financial support from the Dutch Science Organization (ZonMW-NWO Top grant 912-03-047 to BW), the Dutch Cancer Society (KWF grant UM 2003-2821 to BW), and the EU $6^{\text {th }}$ framework program (Euroxy program to BW).

\section{References}

1. Nordsmark M, Bentzen SM, Rudat V, Brizel D, Lartigau E, Stadler P, Becker A, Adam M, Molls M, Dunst J, Terris DJ, Overgaard J: Prognostic value of tumor oxygenation in 397 head and neck tumors after primary radiation therapy. An international multi-center study, Radiother Oncol 2005, 77:18-24

2. Fyles A, Milosevic M, Pintilie M, Syed A, Levin W, Manchul L, Hill RP: Longterm performance of interstial fluid pressure and hypoxia as prognostic factors in cervix cancer, Radiother Oncol 2006, 80:132-137

3. Quennet V, Yaromina A, Zips D, Rosner A, Walenta S, Baumann M, MuellerKlieser $W$ : Tumor lactate content predicts for response to fractionated irradiation of human squamous cell carcinomas in nude mice, Radiother Oncol 2006, 81:130-135

4. Yaromina A, Zips D, Thames HD, Eicheler W, Krause M, Rosner A, Haase $M$, Petersen C, Raleigh JA, Quennet V, Walenta S, Mueller-Klieser W, Baumann $\mathrm{M}$ : Pimonidazole labelling and response to fractionated irradiation of five human squamous cell carcinoma (hSCC) lines in nude mice: the need for a multivariate approach in biomarker studies, Radiother Oncol 2006, 81:122129

5. Semenza GL: Targeting HIF-1 for cancer therapy, Nat Rev Cancer 2003, 3:721-732

6. Maxwell PH, Wiesener MS, Chang GW, Clifford SC, Vaux EC, Cockman ME, Wykoff CC, Pugh CW, Maher ER, Ratcliffe PJ: The tumour suppressor protein VHL targets hypoxia-inducible factors for oxygen-dependent proteolysis, Nature 1999, 399:271-275

7. Arany $Z$, Huang LE, Eckner R, Bhattacharya S, Jiang C, Goldberg MA, Bunn $H F$, Livingston DM: An essential role for p300/CBP in the cellular response to hypoxia, Proc Natl Acad Sci U S A 1996, 93:12969-12973

8. Ruas JL, Poellinger $L$, Pereira $T$ : Functional analysis of hypoxia-inducible factor-1 alpha-mediated transactivation. Identification of amino acid residues critical for transcriptional activation and/or interaction with CREB-binding protein, J Biol Chem 2002, 277:38723-38730

9. Lando D, Peet DJ, Whelan DA, Gorman JJ, Whitelaw ML: Asparagine hydroxylation of the HIF transactivation domain a hypoxic switch, Science 2002, 295:858-861

10. Mahon PC, Hirota K, Semenza GL: FIH-1: a novel protein that interacts with HIF-1alpha and VHL to mediate repression of HIF-1 transcriptional activity, Genes Dev 2001, 15:2675-2686

11. Wouters $B G$, van den Beucken $T$, Magagnin MG, Koritzinsky $M$, Fels $D$, Koumenis $C$ : Control of the hypoxic response through regulation of mRNA translation, Semin Cell Dev Biol 2005, 16:487-501

12. Bennewith $K L$, Durand RE: Quantifying transient hypoxia in human tumor xenografts by flow cytometry, Cancer Res 2004, 64:6183-6189 
13. Cardenas-Navia LI, Yu D, Braun RD, Brizel DM, Secomb TW, Dewhirst MW: Tumor-dependent Kinetics of Partial Pressure of Oxygen Fluctuations during Air and Oxygen Breathing, Cancer Res 2004, 64:6010-6017

14. van den Beucken $T$, Koritzinsky $M$, Wouters $B G$ : Translational control of gene expression during hypoxia, Cancer Biol Ther 2006, 5:749-755

15. Koritzinsky $M$, Seigneuric $R$, Magagnin $M G$, van den Beucken $T$, Lambin $P$, Wouters BG: The hypoxic proteome is influenced by gene-specific changes in mRNA translation, Radiother Oncol 2005, 76:177-186

16. Lu $X$, de la Pena $L$, Barker $C$, Camphausen $K$, Tofilon PJ: Radiation-induced changes in gene expression involve recruitment of existing messenger RNAs to and away from polysomes, Cancer Res 2006, 66:1052-1061

17. Rajasekhar VK, Viale A, Socci ND, Wiedmann M, Hu X, Holland EC: Oncogenic Ras and Akt signaling contribute to glioblastoma formation by differential recruitment of existing mRNAs to polysomes, Mol Cell 2003, 12:889-901

18. Koritzinsky M, Magagnin MG, van den Beucken $T$, Seigneuric $R$, Savelkouls $K$, Dostie J, Pyronnet S, Kaufman RJ, Weppler SA, Voncken JW, Lambin P, Koumenis C, Sonenberg N, Wouters BG: Gene expression during acute and prolonged hypoxia is regulated by distinct mechanisms of translational control, Embo J 2006, 25:1114-1125

19. Liu L, Cash TP, Jones RG, Keith B, Thompson CB, Simon MC: Hypoxia-induced energy stress regulates mRNA translation and cell growth, Mol Cell 2006, 21:521-531

20. Koumenis $C$, Naczki C, Koritzinsky M, Rastani S, Diehl A, Sonenberg $N$, Koromilas A, Wouters BG: Regulation of protein synthesis by hypoxia via activation of the endoplasmic reticulum kinase PERK and phosphorylation of the translation initiation factor eIF2alpha, Mol Cell Biol 2002, 22:7405-7416

21. Bhattacharya $S$, Michels $C L$, Leung $M K$, Arany $Z P$, Kung $A L$, Livingston $D M$ : Functional role of p35srj, a novel p300/CBP binding protein, during transactivation by HIF-1, Genes Dev 1999, 13:64-75

22. Freedman SJ, Sun ZY, Kung AL, France DS, Wagner G, Eck MJ: Structural basis for negative regulation of hypoxia-inducible factor-1alpha by CITED2, Nat Struct Biol 2003, 10:504-512

23. Yin Z, Haynie J, Yang $X$, Han B, Kiatchoosakun S, Restivo J, Yuan S, Prabhakar NR, Herrup $K$, Conlon RA, Hoit BD, Watanabe M, Yang YC: The essential role of Cited2, a negative regulator for HIF-1alpha, in heart development and neurulation, Proc Natl Acad Sci U S A 2002, 99:1048810493

24. Blais JD, Filipenko V, Bi M, Harding HP, Ron D, Koumenis C, Wouters BG, Bell JC: Activating transcription factor 4 is translationally regulated by hypoxic stress, Mol Cell Biol 2004, 24:7469-7482

25. Koritzinsky $M$, Rouschop KM, van den Beucken T, Magagnin MG, Savelkouls $K$, Lambin P, Wouters BG: Phosphorylation of eIF2alpha is required for mRNA translation inhibition and survival during moderate hypoxia, Radiother Oncol 2007, 83:353-361

26. Harding HP, Novoa I, Zhang $Y$, Zeng H, Wek R, Schapira M, Ron D: Regulated translation initiation controls stress-induced gene expression in mammalian cells, Mol Cell 2000, 6:1099-1108

27. Lang KJ, Kappel A, Goodall G]: Hypoxia-inducible factor-1alpha mRNA contains an internal ribosome entry site that allows efficient translation during normoxia and hypoxia, Mol Biol Cell 2002, 13:1792-1801

28. Stein I, Itin A, Einat P, Skaliter R, Grossman Z, Keshet E: Translation of vascular endothelial growth factor mRNA by internal ribosome entry: implications for translation under hypoxia, Mol Cell Biol 1998, 18:3112-3119 
29. Thoma C, Bergamini G, Galy B, Hundsdoerfer P, Hentze MW: Enhancement of IRES-mediated translation of the c-myc and BiP mRNAs by the poly $(\mathrm{A})$ tail is independent of intact eIF4G and PABP, Mol Cell 2004, 15:925-935

30. Schwarzer R, Tondera D, Arnold W, Giese K, Klippel A, Kaufmann J: REDD1 integrates hypoxia-mediated survival signaling downstream of phosphatidylinositol 3-kinase, Oncogene 2005, 24:1138-1149

31. Brugarolas J, Lei $K$, Hurley $R L$, Manning $B D$, Reiling JH, Hafen $E$, Witters LA, Ellisen LW, Kaelin WG, Jr.: Regulation of mTOR function in response to hypoxia by REDD1 and the TSC1/TSC2 tumor suppressor complex, Genes Dev 2004, 18:2893-2904 


\section{CHAPTER 7}

\section{Hypoxia-induced carbonic anhydrase 9 expression is dependent on the unfolded protein response}

Submitted for publication.

Twan van den Beucken, Marianne Koritzinsky, Hanneke

Niessen, Ludwig Dubois, Kim Savelkouls, Hilda Mujcic, Barry Jutten, Juraj Kopacek, Sylvia Pastorekova, Philippe Lambin, Willem Voncken, Kasper M.A. Rouschop and Bradly G.

Wouters. 


\begin{abstract}
Adaptation to tumor hypoxia is mediated in large part by changes in protein expression These are driven by multiple pathways including activation of the HIF-1 transcription factor and the endoplasmic reticulum kinase PERK, a component of the unfolded protein response (UPR). Through gene expression profiling we discovered that induction of the HIF-1 target gene CA9 was defective in mouse embryo fibroblasts derived from mice harboring an eIF $2 \alpha$ S51A knock-in mutation. This finding was confirmed in isogenic human U373 glioblastoma and HCT116 colon carcinoma cells with an engineered defect in eIF $2 \alpha$ phosphorylation. Impaired CA9 expression was not due to changes in HIF activity or CA9 mRNA stability. Using chromatin immunoprecipitation we show instead that the PERK/eIF $2 \alpha$ translationally regulated gene ATF4 binds directly to the CA9 promoter and is associated with loss of the transcriptional repressive histone 3 lysine 27 tri-methylation mark. Transient knock-down or overexpression of ATF4 confirmed its role in CA9 induction during hypoxia. Our data indicate that regulation of CA9 is achieved through independent activation of both the HIF-1 and UPR hypoxia response pathways.
\end{abstract}




\section{Introduction}

Mammalian cells have evolved a number of sophisticated mechanisms to allow adaptation to changes in the supply of essential metabolic factors. These include mechanisms for sensing the presence of nutrients, energy, and oxygen. Many of these mechanisms are exploited by tumor cells to facilitate survival to the unique conditions found within the microenvironment of solid human tumors ${ }^{1}$. In particular, adaptation to hypoxia is considered to be an important factor that contributes to the poor prognosis of patients with high levels of tumor hypoxia ${ }^{2}$. The hypoxia inducible factor (HIF) plays an essential role in this adaptation by transcriptionally regulating the expression of a large number of genes involved in angiogenesis, anaerobic glycolysis, invasion and metastasis ${ }^{3}$.

HIF- 1 is a heterodimeric transcription factor consisting of $\alpha$ and $\beta$ subunits that are both ubiquitously expressed. Under aerobic conditions HIF- $1 \alpha$ is ubiquitinated by the von Hippel-Lindau tumor suppressor protein ( $P V H L$ ) causing its rapid degradation within the $26 \mathrm{~S}$ proteasome ${ }^{4}$. This process relies on hydroxylation of proline residues 402 and 564 by a family of oxygen-sensitive prolyl hydroxylases (PHD1-3). Under hypoxic conditions hydroxylation cannot occur, resulting in HIF-1 $\alpha$ stabilization

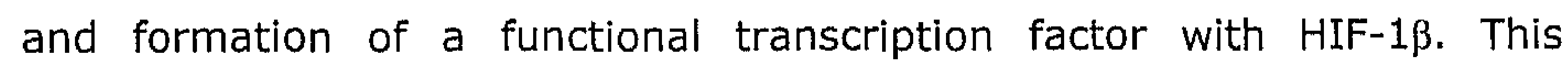
complex drives transcription of HIF responsive genes by binding to hypoxia responsive elements (HRES) in their promoters.

One of the best characterized targets of HIF-1 is carbonic anhydrase (CA) 9. The CA9 promoter contains a single HRE element that is essential for its transcriptional increase during hypoxia ${ }^{5}$. CA9 is frequently over expressed in human tumors and is associated with poor prognosis ${ }^{6,7}$. CA9 belongs to a family of zinc metalloenzymes involved in regulating $\mathrm{pH}$ by catalyzing the reversible conversion of carbon dioxide to bicarbonate and $\mathrm{H}^{+}$. CA9 is found in the plasma membrane with its active site on the extracellular domain. Consequently, it is has been shown to contribute to the acidification of the extracellular tumor environment during hypoxia ${ }^{8}$. At the same time, the bicarbonate produced by this reaction can be used 
by bicarbonate transporters for import to cytoplasm and neutralization of intracellular $\mathrm{pH}$. Low extracellular $\mathrm{pH}$ in tumors is associated with increased invasion and metastasis ${ }^{9,10}$ and can also negatively affect cancer treatment by modulating uptake of anticancer drugs ${ }^{11}$. The potential importance of CA9 as a marker of hypoxia and regulator of tumor $\mathrm{pH}$ has made it an attractive diagnostic and therapeutic target ${ }^{12-14}$.

In addition to regulation by HIF through the HRE element, five potentially important regions within the CA9 promoter have been identified by DNase I footprinting (PR1-PR5) ${ }^{15}$. PR1 plays an important role in CA9 induction during culture at high cell density via binding of SP1/3 transcription factors ${ }^{16}$. In vitro cell culture at high cell density leads to a small decrease in the pericellular oxygen concentration due to high rates of cellular oxygen. Under these conditions, transcriptional activation of CA9 requires cooperation of SP1 with minimal HIF-1 activity and operates through the PI3K pathway ${ }^{16}$. The MAPK/ERK pathway has also been implicated in regulation of CA9 expression in response to both hypoxia and culture at high cell density ${ }^{17}{ }^{18}$. Simultaneous inhibition of PI3K and MAPK pathways is sufficient to prevent CAIX expression during both conditions ${ }^{17}$.

Changes in protein expression during hypoxia also arise through HIF independent mechanisms. Several recent reports indicate that hypoxia activates an adaptive program called the unfolded protein response $(U P R)^{19-21}$. Activation of the UPR during hypoxia results in an immediate but reversible reduction in overall protein synthesis ${ }^{22}$. This is mediated through phosphorylation of the eukaryotic translation initiation factor eIF2 $\alpha$ by the ER resident kinase PERK. Mouse embryonic fibroblasts (MEFs) deficient in PERK or expressing an allele of eIF2 $\alpha$ mutated at the PERK phosphorylation site (S51A) are unable to inhibit translation during hypoxia and demonstrate increased hypoxia sensitivity and profoundly decreased tumor growth compared to their WT controls ${ }^{20}$. Although activation of the UPR causes a global decrease in mRNA translation, the degree of inhibition is highly gene specific ${ }^{22-24}$. The sensitivity of any 
particular mRNA to inhibition of translation is influenced by sequences in its $5^{\prime}$ and $3^{\prime}$ untranslated regions (UTRs). Paradoxically, the translation of some mRNA's, including the transcription factor ATF-4, are stimulated under conditions where eIF $2 \alpha$ is phosphorylated ${ }^{22,23}$.

In this study we investigated the effect of UPR dependent signaling during hypoxia on the regulation of CA9. We found that CA9 expression is regulated through a PERK/eIF2 $\alpha /$ ATF4 dependent pathway. We show that ATF4 directly binds to the CA9 promoter and is required for transcriptional induction of CA9 during hypoxia. Our data indicate that regulation of CA9 is achieved through independent activation of both the HIF-1 and UPR hypoxia response.

\section{Materials and Methods}

\section{Cell culture}

Exponentially growing U373 (human glioblastoma-astrocytoma) and HCT116 (human colon carcinoma) cells were grown in Mem alpha and DMEM respectively, supplemented with $10 \%$ foetal calf serum (FCS) (Sigma-Aldrich). Mouse embryonic fibroblasts (MEFs) wild type or with a homozygous knock-in mutation for eIF $2 \alpha(S 51 A){ }^{25}$ were grown in DMEM plus 10\% FCS and supplemented with $55 \mu \mathrm{M} \beta$-mercaptoethanol and nonessential amino acids. For hypoxic exposure cells were transferred into a MACS VA500 microaerophilic workstation (Don Whitley Scientific, Shipley, UK). The atmosphere in the chamber consisted of $5 \% \mathrm{H} 2,5 \% \mathrm{CO}, 0 \%$ $\mathrm{O} 2$, and $90 \% \mathrm{~N} 2$.

\section{RNA extraction and quantitative RT-PCR}

RNA was isolated using SV Total RNA Isolation System (Promega) according manufacturers' instructions. RNA samples were reverse transcribed using iScript kit as described by manufacturer (Biorad). Realtime PCR was performed in ABI 7500 (Applied Biosystems). The abundance of human and mouse transcripts were determined using 
Taqman Gene Expression Arrays (Applied Biosystems) or with primers in combination with SYBR Green I (Applied Biosystems) as described in supplementary material and methods. Total mRNA samples were normalized by $18 \mathrm{~S}$ rRNA. For determination CA9 mRNA half life cells were treated with $5 \mu \mathrm{g} / \mathrm{ml}$ actinomycinD (Sigma) for the indicated times.

\section{Western blot analysis}

Cells were washed twice with cold PBS and scraped in $50 \mathrm{mM}$ Tris $\mathrm{HCl} \mathrm{pH}$ $8.0,150 \mathrm{mM} \mathrm{NaCl}, 1 \% \mathrm{NP}-40,0.5 \%$ sodium deoxycholate and $0.1 \%$ SDS supplemented with protease inhibitors (Roche). After centrifugation at $10,000 \mathrm{~g}$ supernatants were boiled in Laemmli buffer for $10 \mathrm{~min}$ and proteins were resolved by SDS-PAGE. Proteins were subsequently transferred onto nitrocellulose membranes and blocked overnight at $4^{\circ} \mathrm{C}$ in PBS containing $0.1 \%$ Tween-20 (PBS-T) supplemented with $5 \%$ skim milk powder. Membranes were probed for $1 \mathrm{~h}$ with antibodies directed against CAIX (M75 ${ }^{26}$ ) , HIF-1 $\alpha$ (Transduction Laboratories) or $\beta$-actin (Sigma). Bound antibodies were visualized using HRP-linked secondary antibodies (anti-rabbit (Cell Signaling Technologies) and anti-mouse (Sigma)) and ECL luminescence (Pierce).

\section{Transfection of reporter constructs and SiRNA}

U373 and MEF cells were transiently cotransfected with CA9 promotor constructs and pCDNALacZ using Lipofectamine (Invitrogen) as described by the manufacturer. Transfected cells were sub cultured $16 \mathrm{~h}$ post transfection, exposed to hypoxia and finally harvested 48 hrs after transfection. Luciferase and $\beta$-galactosidase activity was measured using commercial luciferase kit (Promega) and galacto light system kit (Applied Biosystems) in a LB9507 luminometer (Berthold). For transient knock down U373 cells were transfected with 100nM siRNA duplexes using Oligofectamine according to the manufacturers' instructions. siRNA duplex targeting ATF4 122372, CHOP 146321, ATF6 115889 and HIF1 42840 was obtained from Ambion and scramble control siRNA was from 
Dharmacon. Transfected cells were sub cultured $24 \mathrm{~h}$ post transfection and harvested 72 hrs after transfection. The ATF4 open reading frame was amplified from PCMV_XL5 (Origen) using following primers ATF4_FW 5'gtatgcggaggatccccgcaacatgaccgaaatgagc- $3^{\prime}$ and ATF4_RV 5'tccgcatacgaattcctcctgactatcctcaactagg $-3^{\prime}$ and cloned into PCDNA5 (Invitrogen) using BamHI and ECORI restriction sites. Transfection of U373 cells with pCDNA5-ATF4 or empty vector control was done as for the reporter constructs described above.

\section{Chromatin immunoprecipitation assays}

ChIPs were performed and analyzed essentially as described previously ${ }^{27}$, see supplementary materials and methods.

\section{Tumor xenograft growth}

Animal experiments were performed using adult NMRI ( $\mathrm{nu} / \mathrm{nu}$ ) female mice $(28-32 \mathrm{~g})$ from the animal facility of the Catholic University of Leuven in Belgium. The animal facilities and experiments were in accordance with local institutional guidelines for animal welfare and were approved by the Animal Ethics Committee of the university. Three million U373 pcDNA3, U373 HIF- $1 \alpha$ and U373 S51A cells were resuspended in $100 \mu \mathrm{l}$ growth medium and injected sub-cutaneously in to the lateral flank of recipient mice. Tumors were excised after they reached approximately $100-300 \mathrm{~mm}^{3}$ and fixed in 1-4\% formaldehyde and embedded in paraffin.

\section{Immunohistochemical analysis of CAIX}

$5 \mu \mathrm{m}$ sections were deparaffinized in xylene, rehydrated in a graded alcohol series and finally with water. Endogenous peroxidase was quenched using $0.3 \%$ hydrogen peroxide in absolute methanol. Epitope retrieval was done by microwave treatment in the presence of $10 \mathrm{mM}$ sodium citrate $(\mathrm{pH} 6.0)$. Sections were incubated with rabbit polyclonal anti CAIX antibody (1:1000 dilution, Novus) for $60 \mathrm{~min}$ at RT. A secondary biotinylated polyclonal goat anti rabbit antibody (1:500 dilution, 
Dakocytomation) was applied, followed by incubation with the vectastain $A B C$ kit (1:200 dilution, Vector Laboratories). Staining was visualized using DAB chromogen (Dako). Sections were rinsed and counterstained with hematoxylin (Klinipath).

A

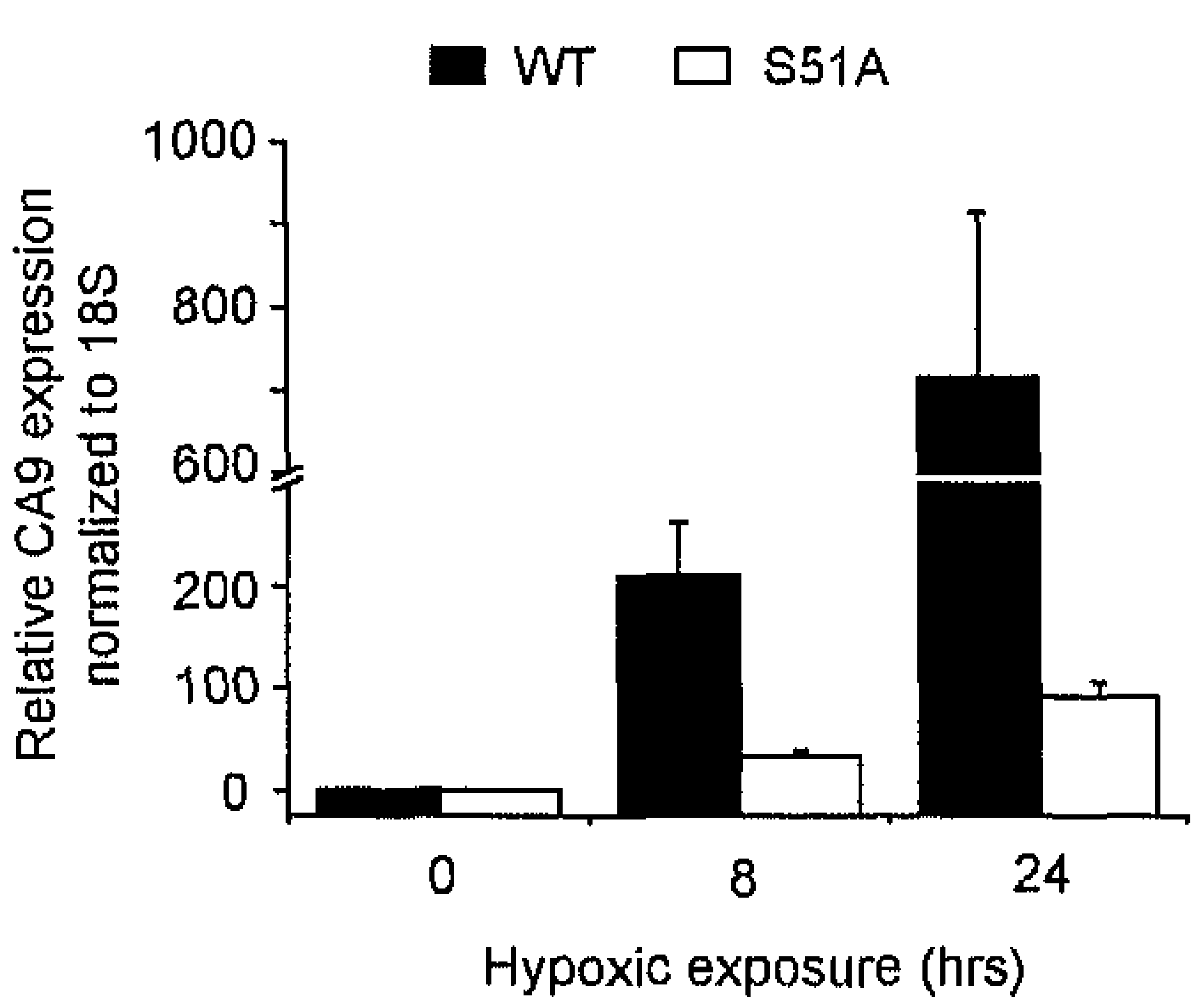

C

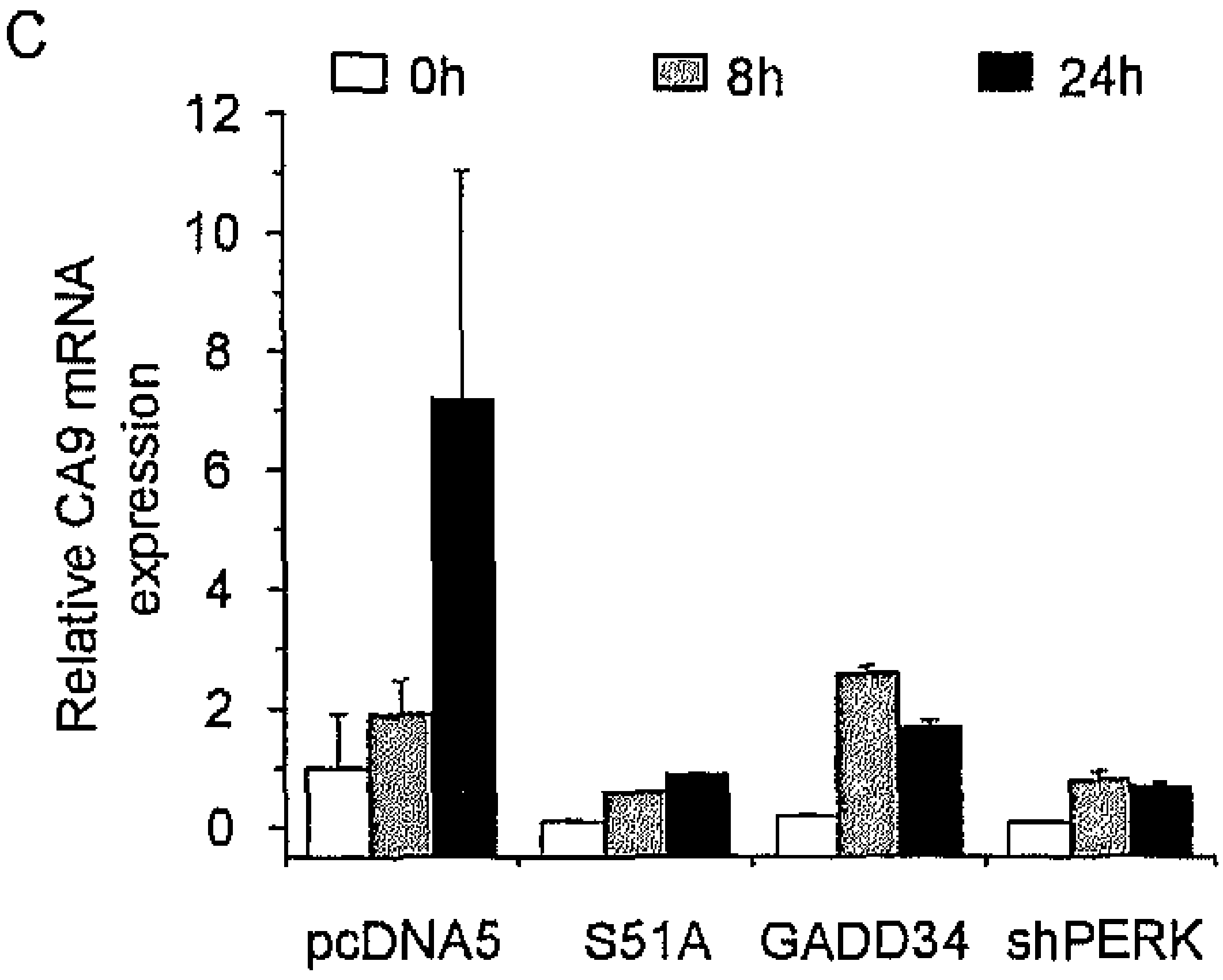

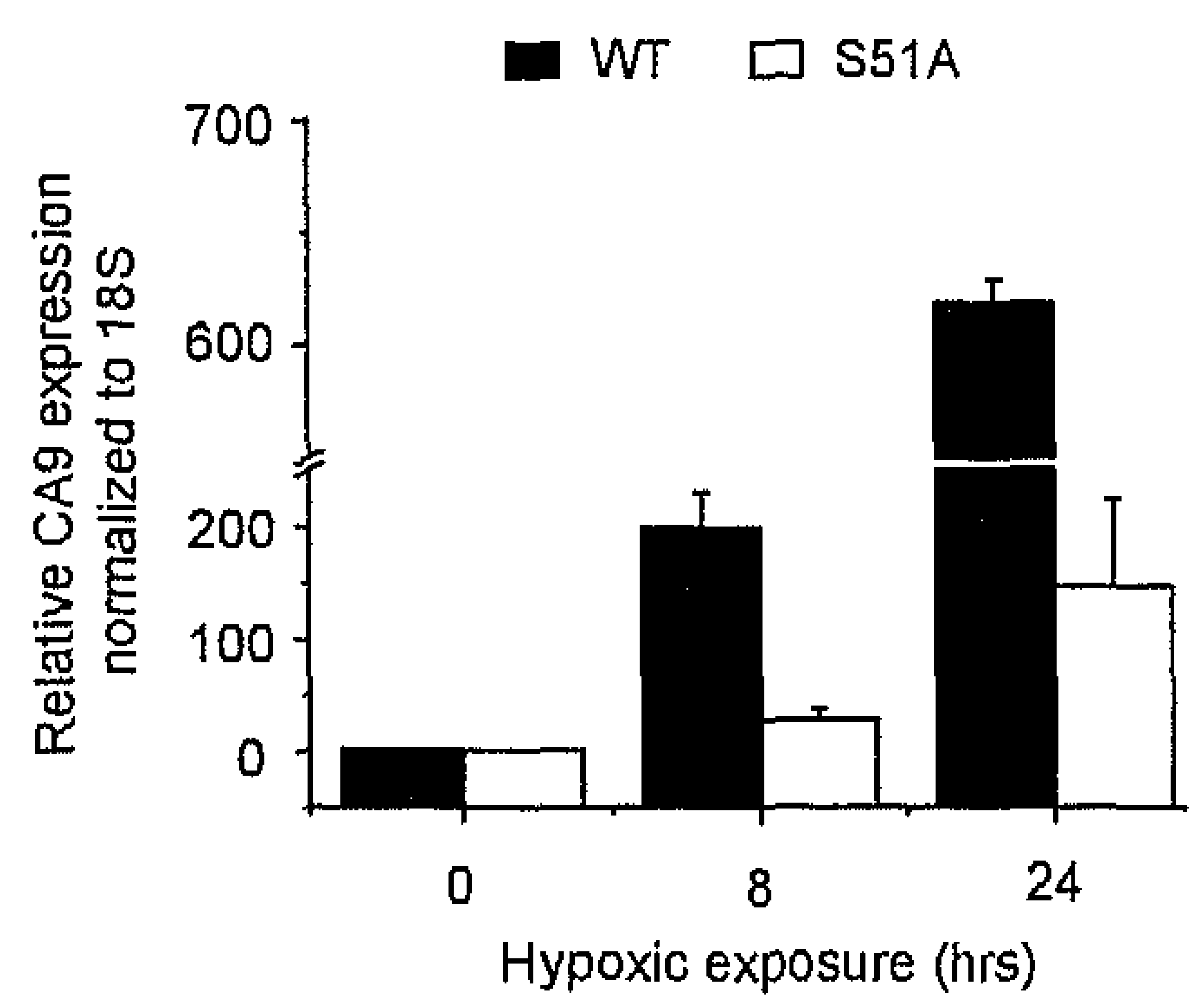

D

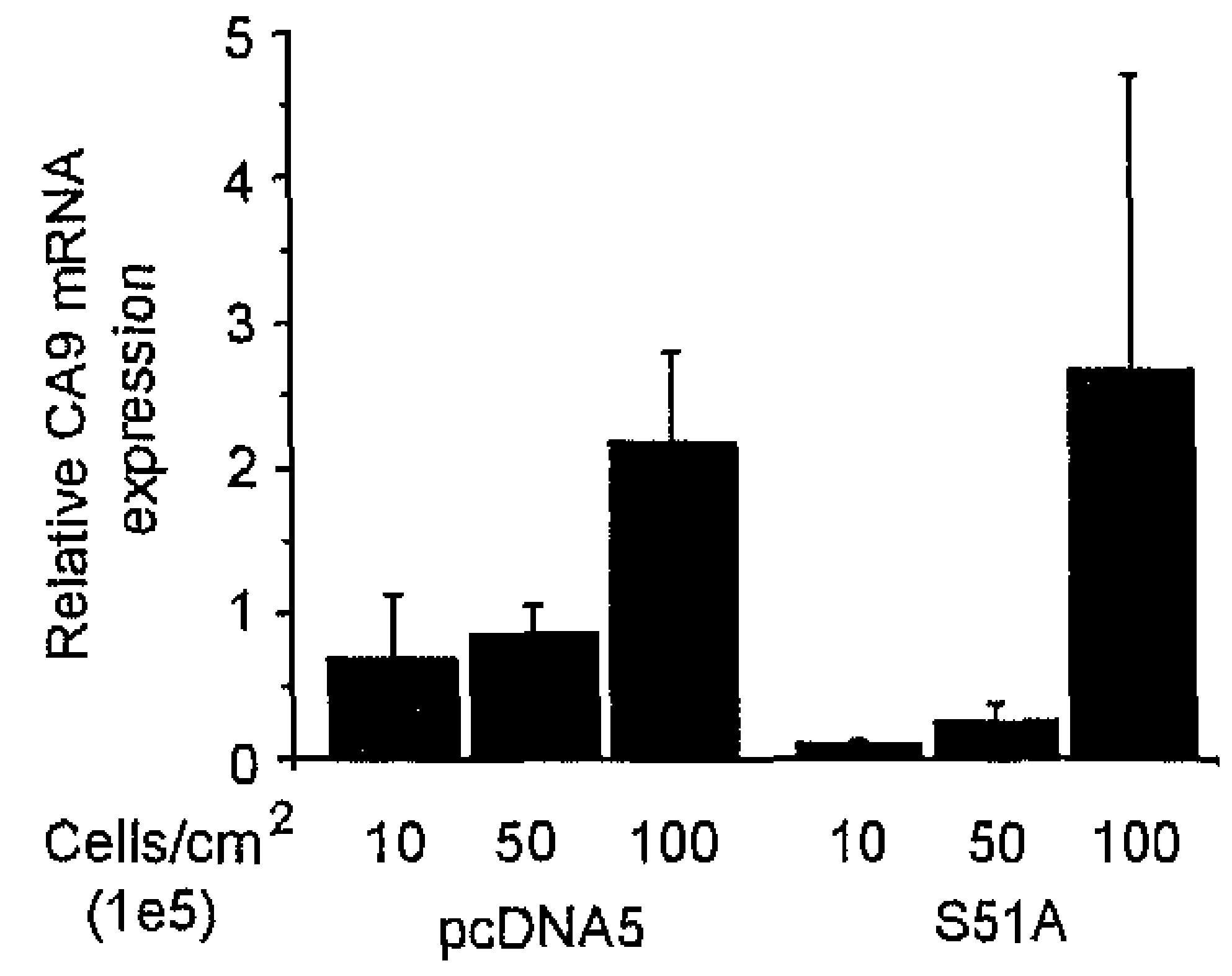

E

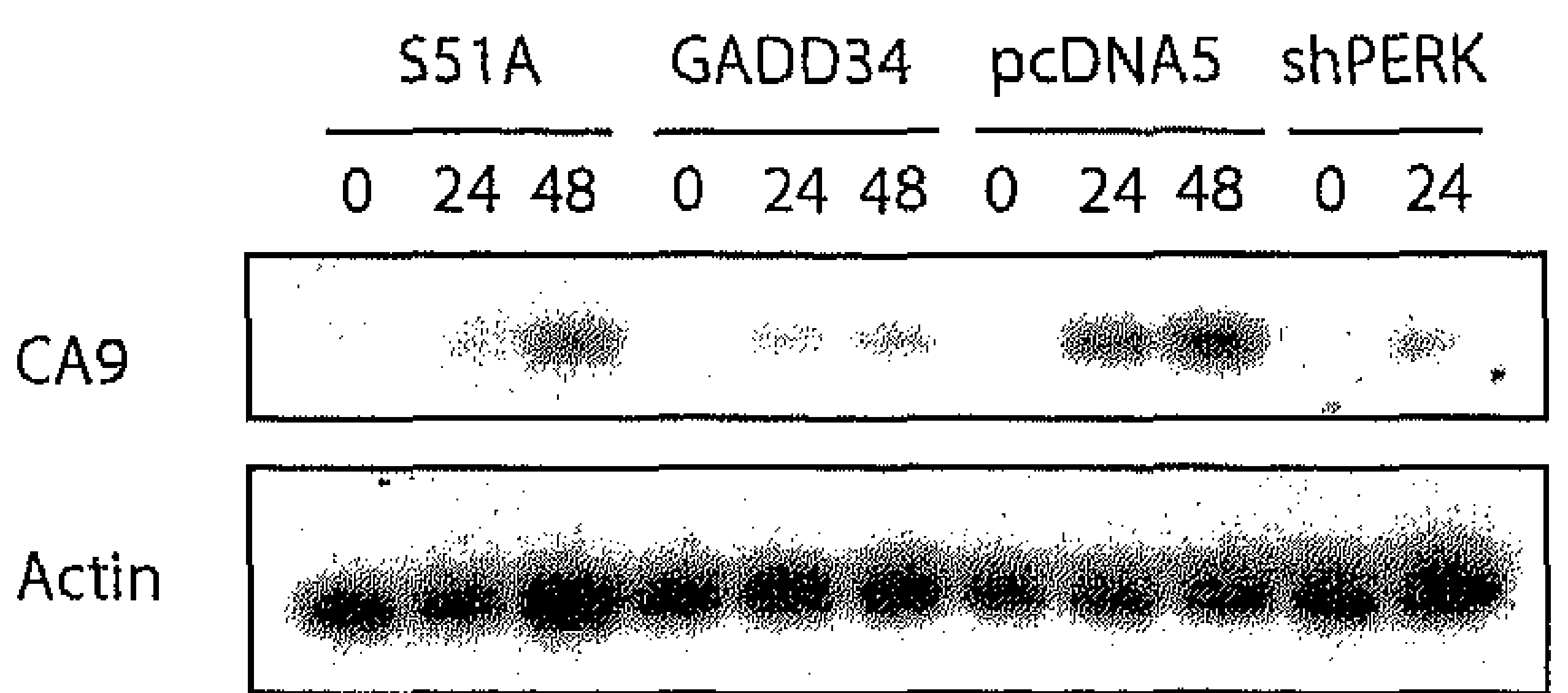




\section{Results}

\section{Hypoxic induction of CA9 requires eIF $2 \alpha$ phosphorylation}

In an ongoing screen to characterize changes in gene expression during hypoxia mediated by the UPR we compared transcriptional profiles of wildtype and eIF2 $\alpha$ S51A mutant MEFs. We identified CA9 as one of the top differentially regulated genes between the WT and S51A cells which are unable to phosphorylate eIF $2 \alpha^{25}$ (data not shown). Quantitative RT-PCR analysis confirmed that CA9 mRNA levels during hypoxia are approximately 5 to 8 -fold lower in the S51A MEFs compared with their WT controls (Figure $1 \mathrm{~A}+\mathrm{B}$ ). The reduction in $\mathrm{CA9}$ expression in the $551 \mathrm{~A}$ mutants is observed during culture under both severe $\left(<0.02 \% \mathrm{O}_{2}\right)$ and more moderate $\left(0.2 \% \mathrm{O}_{2}\right)$ hypoxia.

We also assessed hypoxia induced CA9 expression in a series of human cell lines engineered to have defects in eIF $2 \alpha$ phosphorylation. We created isogenic stable cell lines expressing different constructs at a single genomic site that effectively prevent eIF $2 \alpha$ phosphorylation (Rouschop and Wouters, in preparation) (data not shown). These included expression of a dominant negative eIF2 $\alpha(\mathrm{S} 51 \mathrm{~A})^{28}$ that is insensitive to PERK signaling, a C-terminal fragment of GADD34 that promotes dephosphorylation of eIF $2 \alpha$, and a shRNA against PERK (shPERK). Figure $1 C$ shows that expression of each of these three constructs in U373 glioblastoma cells effectively prevents induction of CA9 during hypoxia.

Figure 1. CA9 expression is impaired in cells defective in eIF $2 \alpha$ phosphorylation during hypoxic conditions. WT and S51A MEFs were exposed to severe $\left(0.0 \% \mathrm{O}_{2}\right)$ (A) or moderate $\left(0.2 \% \mathrm{O}_{2}\right)(B)$ hypoxia and CA9 mRNA levels were assessed using Q-PCR after 0,8 and 24 hrs. (C) CAIX levels measured in isogenic U373 cells expressing dominant negative eIF2 $\alpha$ (S51A), GADD34 or shPERK after 0,8 and 24 hrs of severe hypoxia. (D) U373 pCDNA5 and S51A were seeded at different densities and analyzed with Q-PCR for CA9 mRNA expression. (E) CA9 protein expression was assesses by western blotting in isogenic U373 cell lines after exposure to hypoxia. 
A

wT $\square \mathrm{S} 51 \mathrm{~A}$

B

- WT $\square$ S51A

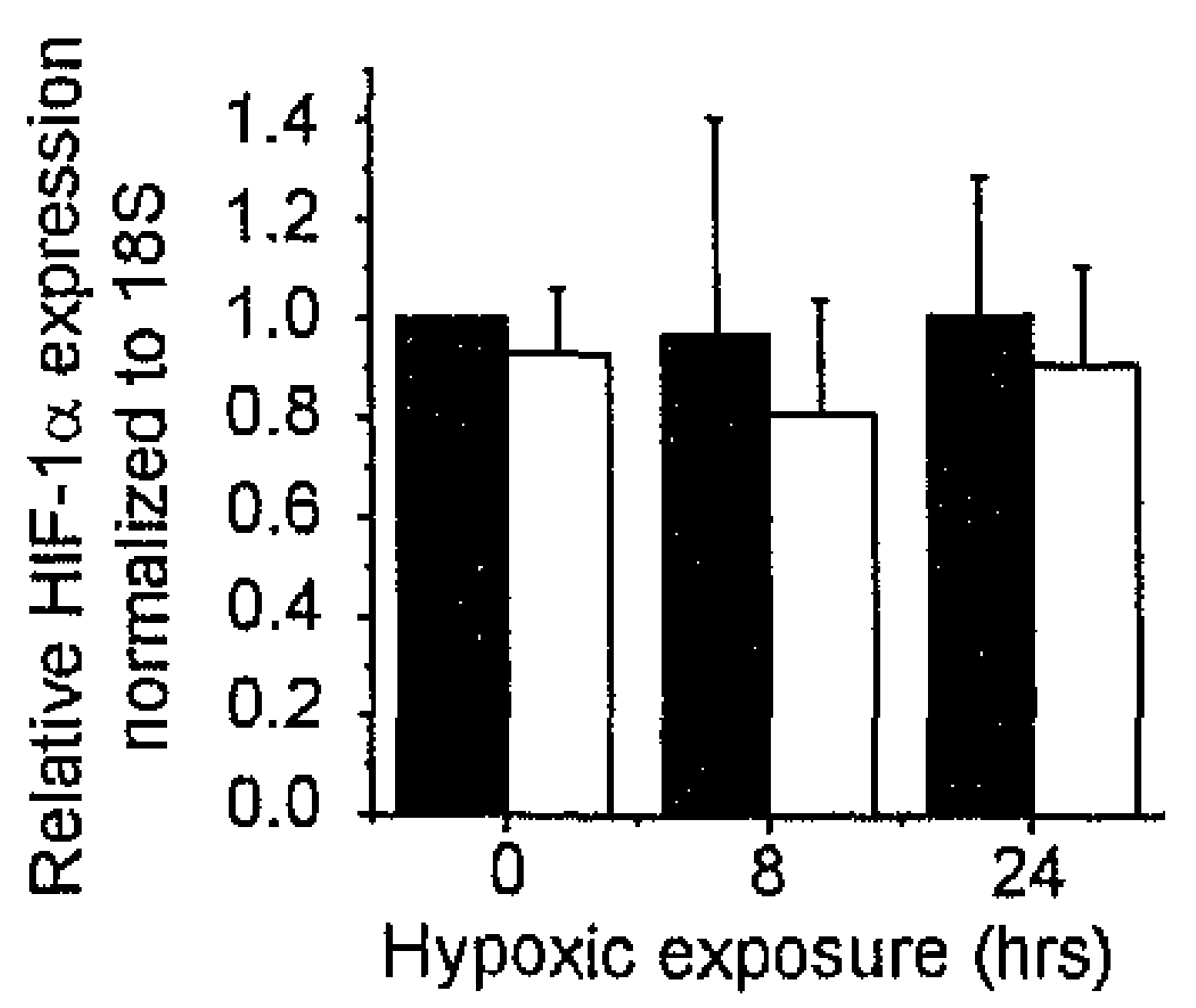

C
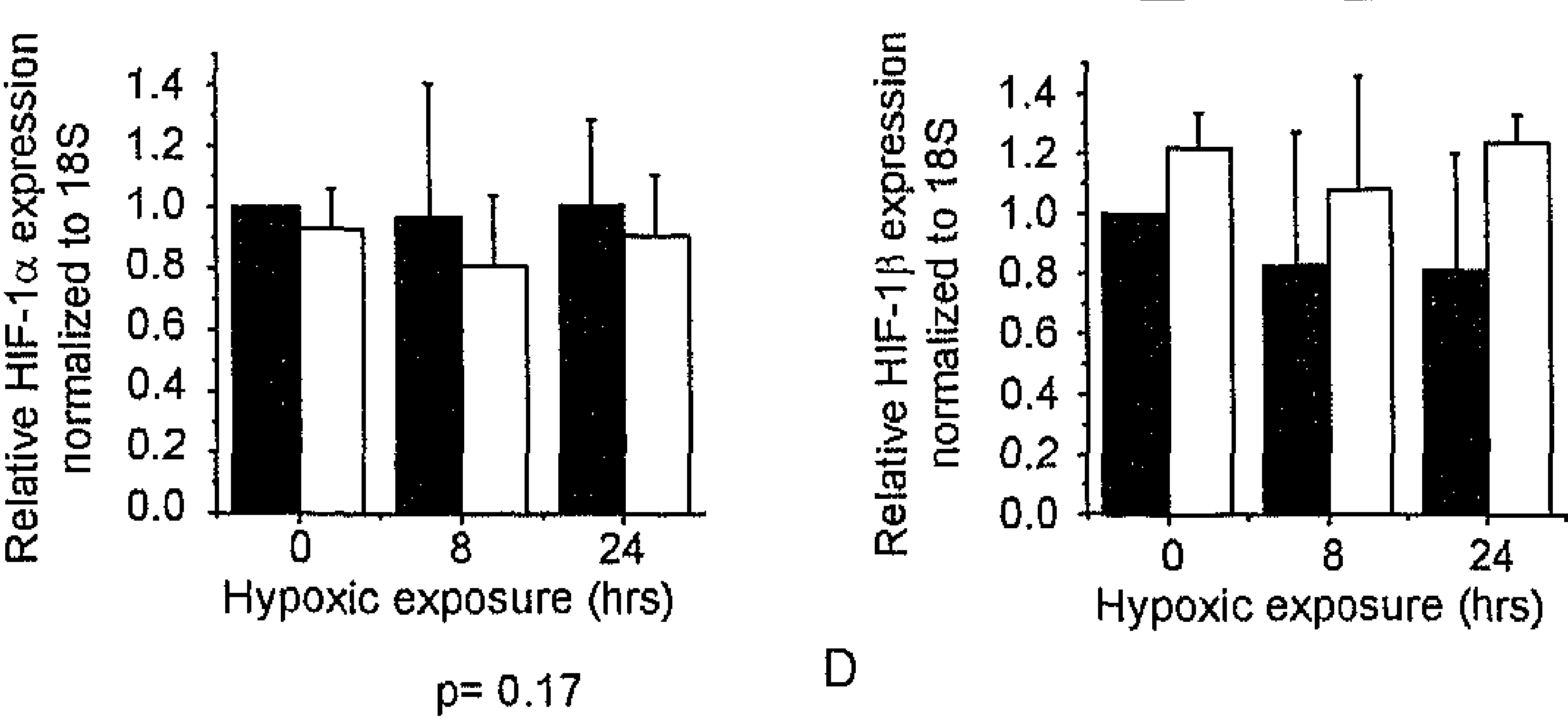

D

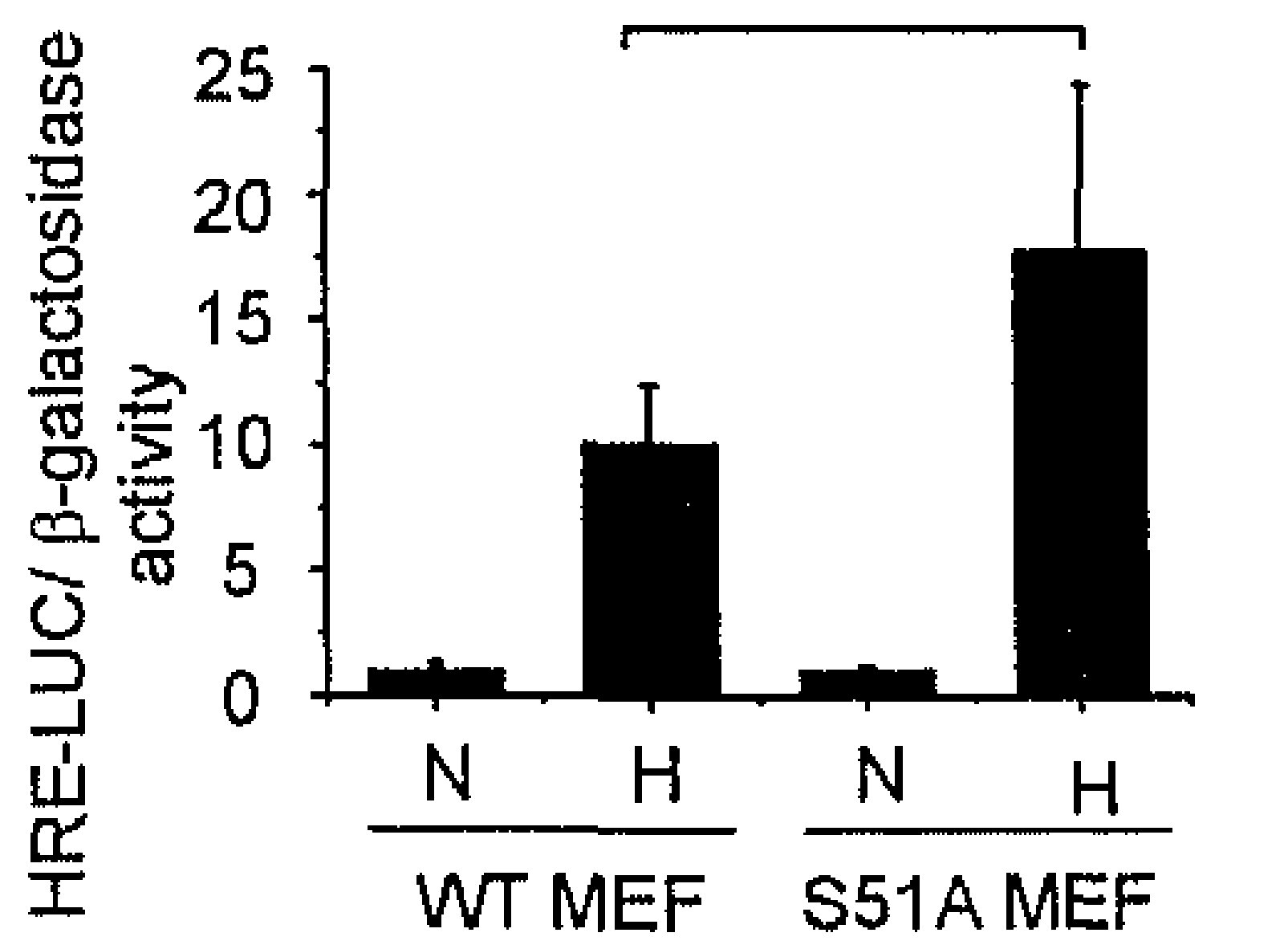

S51A GADD34 pCDNA5 ShPERK

HIF-Io

$\overline{02448} \overline{02448} \overline{02448} \overline{024}$

(

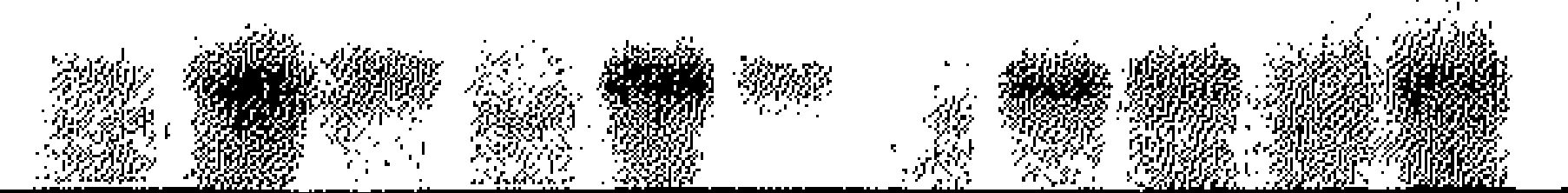

elF4E

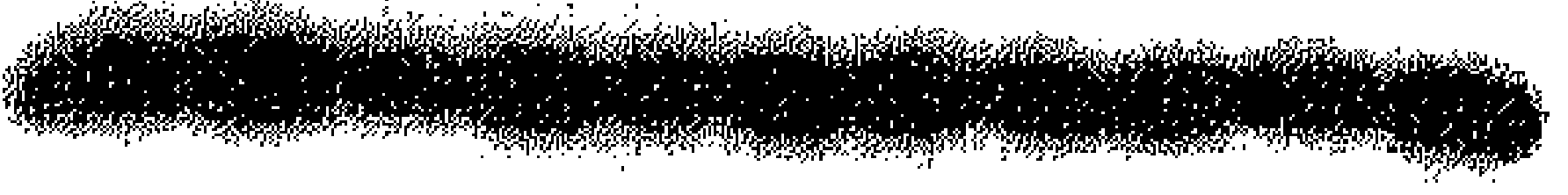

E
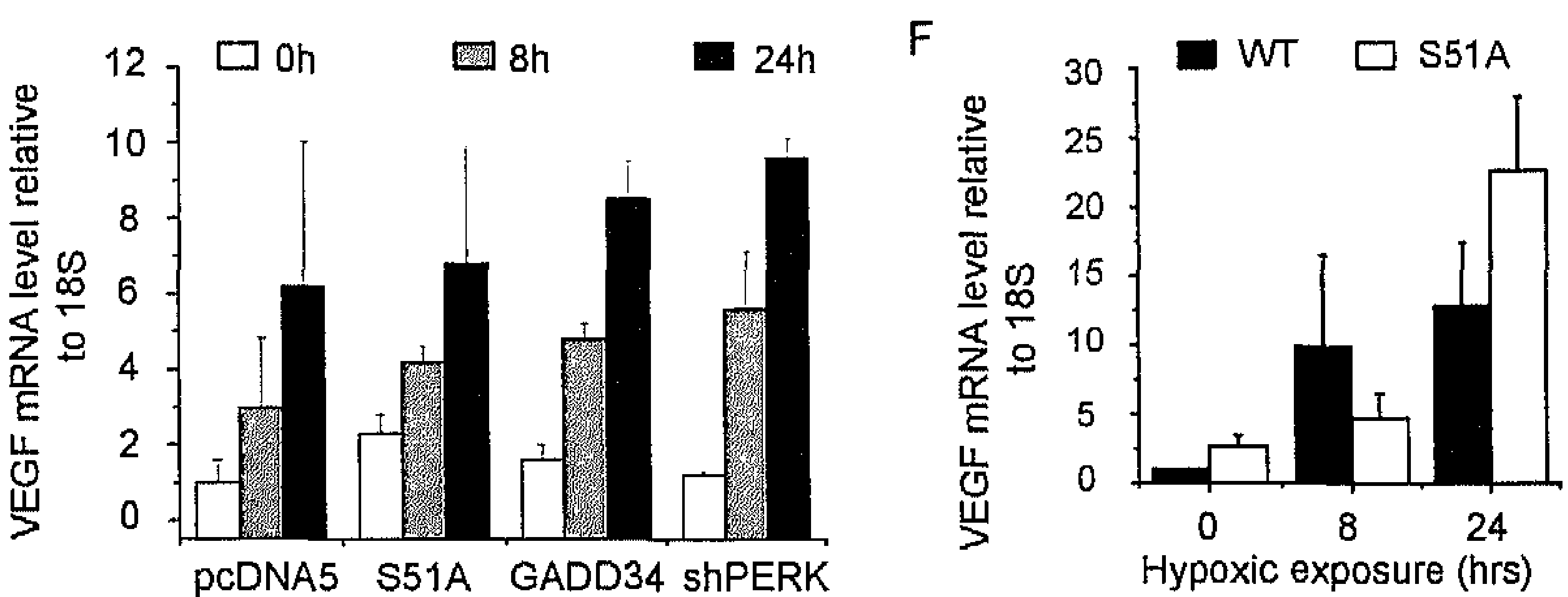

G
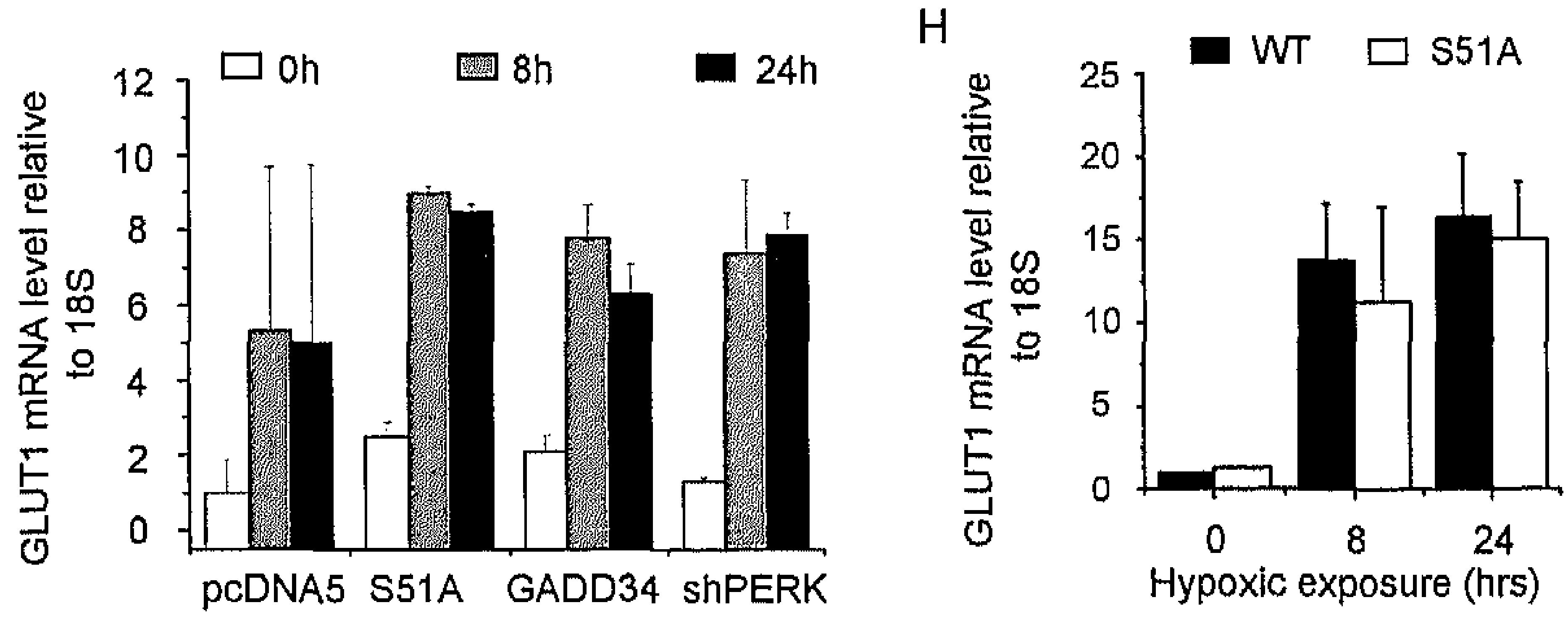
Similar results were observed in HCT116 cells carrying the same constructs (data not shown). The requirement for eIF $2 \alpha$ phosphorylation seems specific to hypoxia since upregualation of CA9 during culture at high cell density under aerobic conditions is similar in wild type and S51A expressing cells (Figure 1D). Importantly, the reduction in CA9 MRNA levels in the eIF $2 \alpha$ phosphorylation deficient cells also results in reduced CA9 protein expression during hypoxia (Figure 1E).

\section{The effects of eIF $2 \alpha$ phosphorylation on CA9 are independent of HIF}

Since the transcription of CA9 is strongly regulated by the HIF-1 transcription factor, we investigated the possibility that HIF-1 signaling was aberrant in cells with defective eIF $2 \alpha$ phosphorylation. However, no differences in the levels of HIF-1 $\alpha$ (Figure $2 A$ ) or HIF-1 $\beta$ (Figure $2 B$ ) mRNA were observed between S51A MEF and their WT controls. HIF activity as assessed using a HRE luciferase reporter plasmid was also similar in the two cell lines (Figure $2 \mathrm{C}$ ). We additionally assessed HIF-1 $\alpha$ protein levels in the human isogenic $U 373$ cell lines after exposure to hypoxia and found similar levels in the three eIF $2 \alpha$ defective cell lines as in the empty vector control (Figure 2D). Finally, we assessed the transcriptional induction of two additional endogenous HIF dependent genes, GLUT-1 and VEGF. In contrast to CA9, the expression of both VEGF (Figure 2E+F) and GLUT-1 (Figure $2 \mathrm{G}+\mathrm{H}$ ) was unaffected in the cell lines with defective eIF2 $\alpha$ phosphorylation. Together these data suggest that HIF-1 activity is not affected by elf $2 \alpha$ phosphorylation.

Figure 2. HIF-1 expression and activity does not require UPR signaling. Analysis of HIF-1 $\alpha$ (A) and HIF-1 $\beta$ (B) mRNA levels in WT and S51A MEF cells. (C) HIF activity was measured in WT and S51A MEF cells using HRE-luciferase constructs normalized to co-transfected $\beta$-galactosidase. (D) Western blot analysis of HIF- $1 \alpha$ levels in isogenic U373 cell lines. Induction of HIF-1 $\alpha$ target genes VEGF (E) and GLUT- 1 (G) in U373 cells after 0,8 and 24 hrs of hypoxia and VEGF (F) and GLUT-1 $(\mathrm{H})$ in WT and S51A MEF cells measured by Q-PCR. 


\section{eIF2 $\alpha$ phosphorylation is required but not sufficient for CA9 induction}

These data suggested that activation of PERK and phosphorylation of eIF2 $\alpha$ contributes to CA9 transcription during hypoxia. We therefore investigated whether other activators of PERK and the UPR might also regulate CA9. To this end we induced ER stress in U373 cells by treatment with either with DTT or tunicamycin. Although both drugs clearly elicited a robust UPR response, as demonstrated by induction of the UPR responsive gene CHOP (Figure 3), neither affected CA9 mRNA levels.

\section{CA9 is regulated at the transcriptional level by ATF4}

In order to explain the reduced CA9 mRNA levels in UPR impaired cells we first examined whether mRNA stability was dependent upon eIF $2 \alpha$ phosphorylation. To this end we determined CA9 mRNA half life after blocking transcription with actinomycin $D$ for various lengths of time. However, the results presented in Figure $4 A$ (MEFs) and $B$ (U373) indicate that CA9 mRNA half life not altered in the eIF2 $\alpha$ phosphorylation deficient cells. These data suggest that differences in CA9 expression are likely due to changes in transcription. Therefore we investigated CA9 promoter activity using luciferase reporter constructs. Promoter region -174 to +37 (pGL3-PR5) contains the 5 previously identified regulatory promoter elements (PR1-PR5) and a hypoxia responsive element (HRE) that influence transcriptional activation of $\mathrm{CA} 9^{5},{ }^{15}$. The minimal promoter region necessary for hypoxia induced expression of CA9 is -50 to +37 (pGL3-PR1). Essential for this are the SP1 transcription factor in PR1 and the HRE immediately downstream of PR1. In our experiments both promoter sequences $(-174$ to +37$)$ and $(-50$ to +37$)$ showed basal levels of activity in UPR impaired cells and their controls under normoxic conditions (Figure 5A). However exposure to hypoxia resulted in a significant induction of promoter activity in all cell lines examined. Furthermore, mutations in either the SP1 binding site or the HRE element completely abolished hypoxia inducebility. The fact that we did not 
observe any differences between UPR impaired cells and control suggests either that elements outside the $(-174$ to +37$)$ promoter region are responsible for the UPR dependent effect on CA9 expression or that the UPR does not influence transcription of these non-chromatin containing plasmids.

tunicamycin
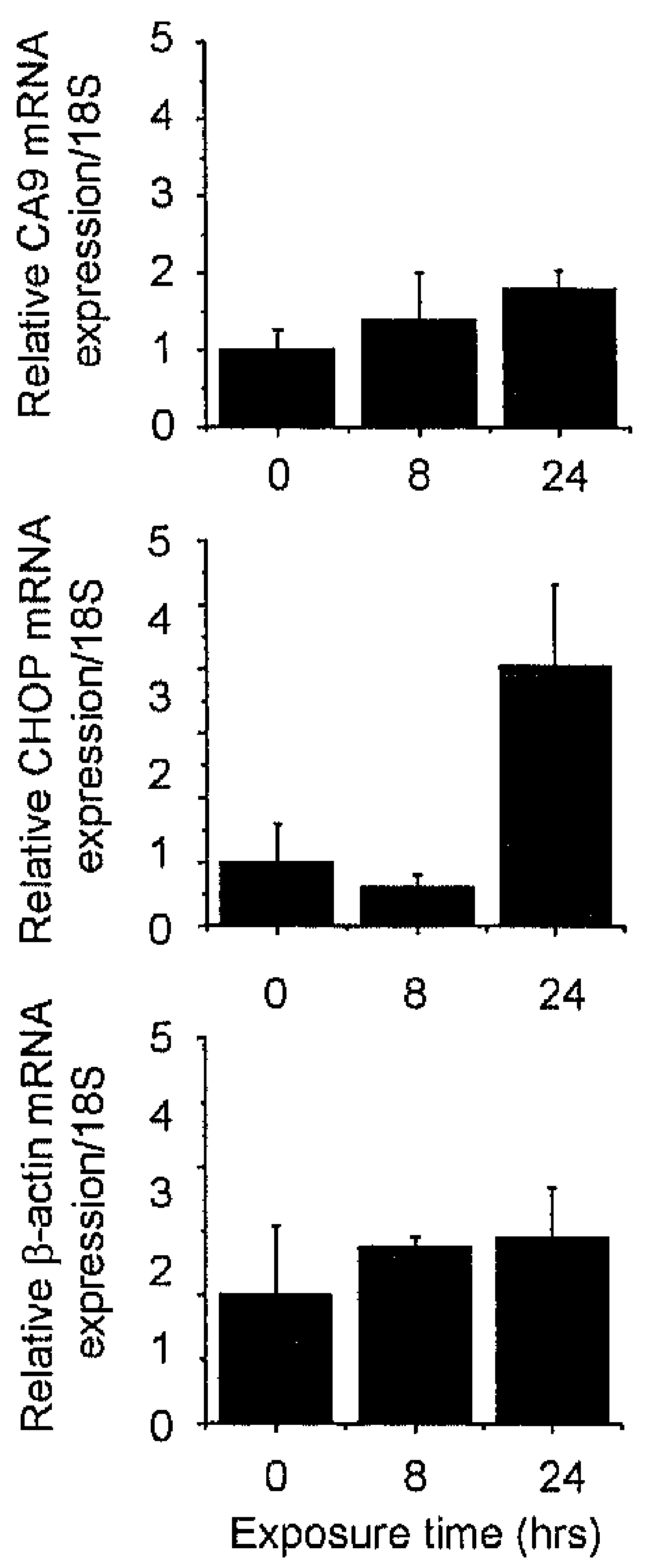

DT
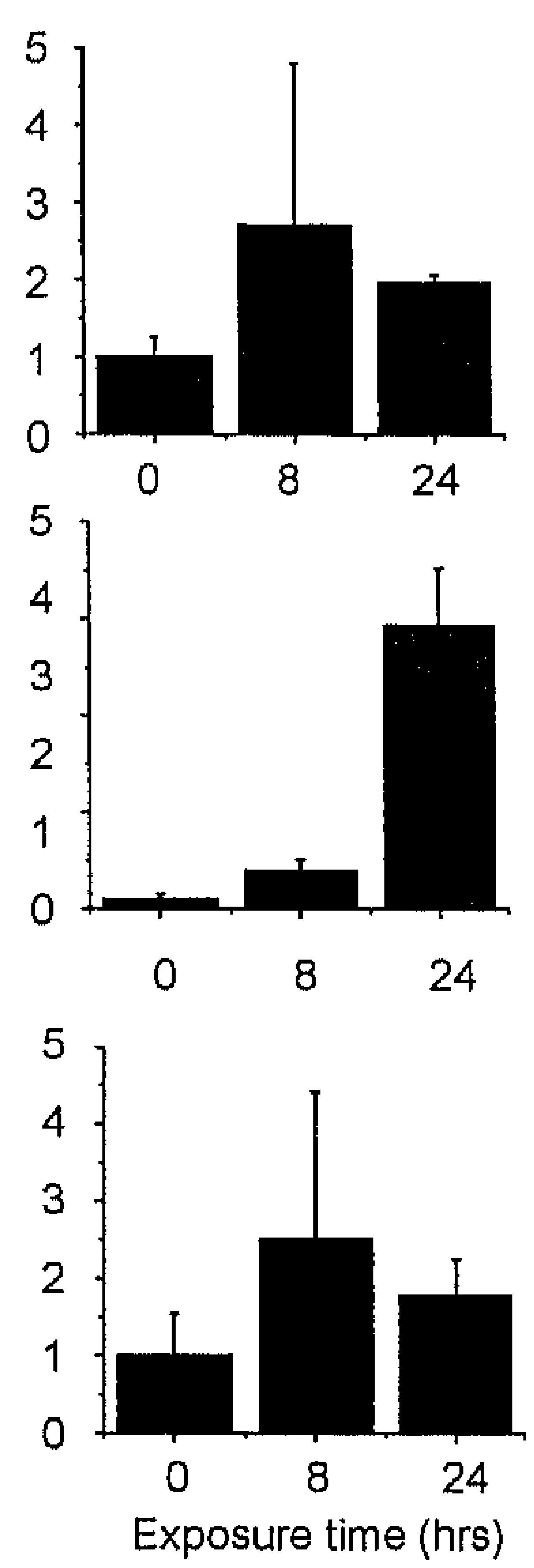

Figure 3. ER stress does not induce CA9 expression during normoxia. U373 cells were stimulated with $1 \mu \mathrm{g} / \mathrm{ml}$ tunicamycin or $1 \mathrm{mM} \mathrm{DTT}$ for 8 or $24 \mathrm{hrs}$. Expression levels for CA9, CHOP and actin were determined using Q-PCR. Bars represent average expression of at least two independent experiments plus standard deviation. 


\section{ATF4 binds the CA9 promoter}

Since our reporter constructs did not cover the complete promoter we attempted to identify transcription factors that are able to bind the CA9 promoter using chromatin immunoprecipitation (ChIP). Using this approach we tested three transcription factors that are known to be activated by the UPR, ATF4, CHOP and ATF6. ATF4 is induced at the translational level in response to PERK dependent phosphorylation of eIF $2 \alpha$ and has also been shown to occur during hypoxia ${ }^{23,29}$. CHOP is a transcriptional target of both ATF4 and ATF6 and is also preferentially translated during hypoxia 22,29 . Interestingly, $\mathrm{CHOP}$ has previously been implicated in the regulation of CA VI ${ }^{30}$. ATF6 on the other hand is also

A

Figure 4. Stability of CA9 mRNA is not dependent on eIF $2 \alpha$ phosphorylation. (A) CA9 MRNA levels were induced in WT and S51A MEF cells with $100 \mu \mathrm{M}$ cobalt chloride for 16 hours after which CA9 mRNA levels were assessed using Q-PCR at different time points after addition of actinomycin D. (B) CA9 mRNA stability in U373 pCDNA5 and U373 S51A cells was determined after exposure to 16 hours of hypoxia.
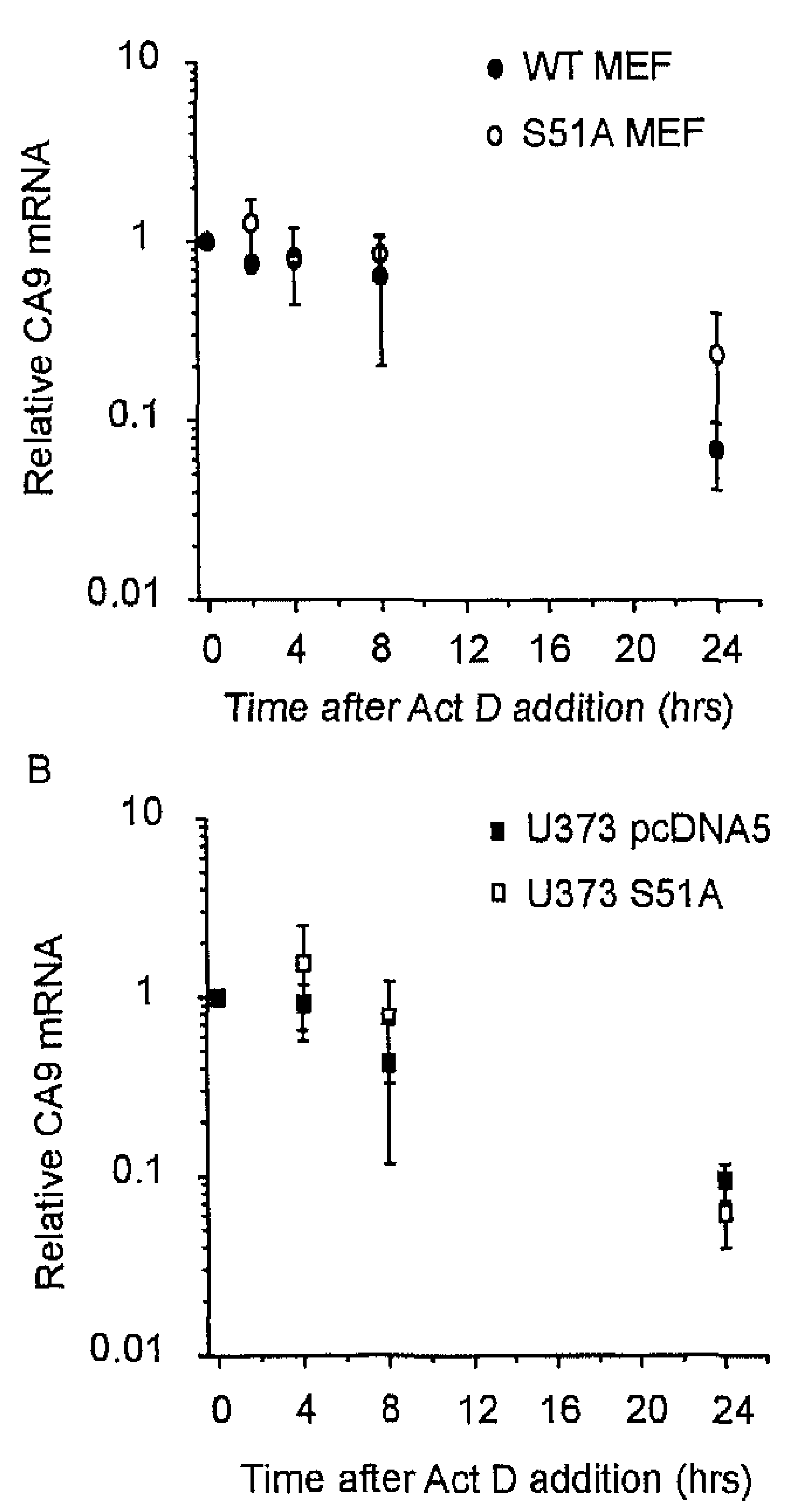
The UPR contributes to hypoxia-induced expression of CA9

induced by the UPR, but has no known dependency upon PERK or eIF2 $\alpha$ phosphorylation. U373 and HCT116 cells were exposed to aerobic or hypoxic conditions $(4 \mathrm{~h})$ after which ChIPs were performed using antibodies against ATF4, CHOP, ATF6 and the HA epitope tag. We observed enrichment of the CA9 promoter after performing the ChIP procedure for ATF4 in U373 (Figure 5B) and HCT116 (Figure 5C). In contrast, there was no enrichment using CHOP or ATF6. ATF-4 binding could be explained by the presence of 2 putative amino acid response elements (AARES) in the CA9 promoter region that closely resemble the ATF-4 DNA binding sequence in the CHOP promoter (ATTGCATCA) ${ }^{31}$ (Figure 5D). Furthermore we show that we could efficiently immuno precipitate the CA9 promoter using an antibody directed against HIF-1 $\alpha$ (Figure 5B). As additional controls for the ChIP procedures we determined the enrichment of known interacting promoter regions over that of an unrelated promoter region of ATG12. The ATF4 and ATF6 ChIPs effectively pulled down the CHOP promoter (up to 28-fold and 6-fold enrichment respectively) and CHOP pulled down the GADD34 promoter ( $\sim 5$-fold enriched) (Figure 5E). We also performed ChIP analysis using an antibody directed against the histone $\mathrm{H} 3$ methylation mark on lysine 27 (H3K27me). The presence of H3K27me correlates with repression of gene expression ${ }^{32}$. With U373 cells cultured under aerobic conditions we obtained significant enrichment of the CA9 promoter using an antibody against the H3K27me mark compared to an unrelated control antibody (Figure 5F). Strikingly this H3K27me mark at the CA9 promoter disappears rapidly upon exposure to hypoxia. Similar results were obtained using HCT116 cells (data not shown). These data suggest that ATF4 mediates endogenous transcriptional activation of CA9 by direct binding to its promoter and that activation occurs following a change in histone methylation. 
A

$\square$ Oh

a $24 \mathrm{~h}$

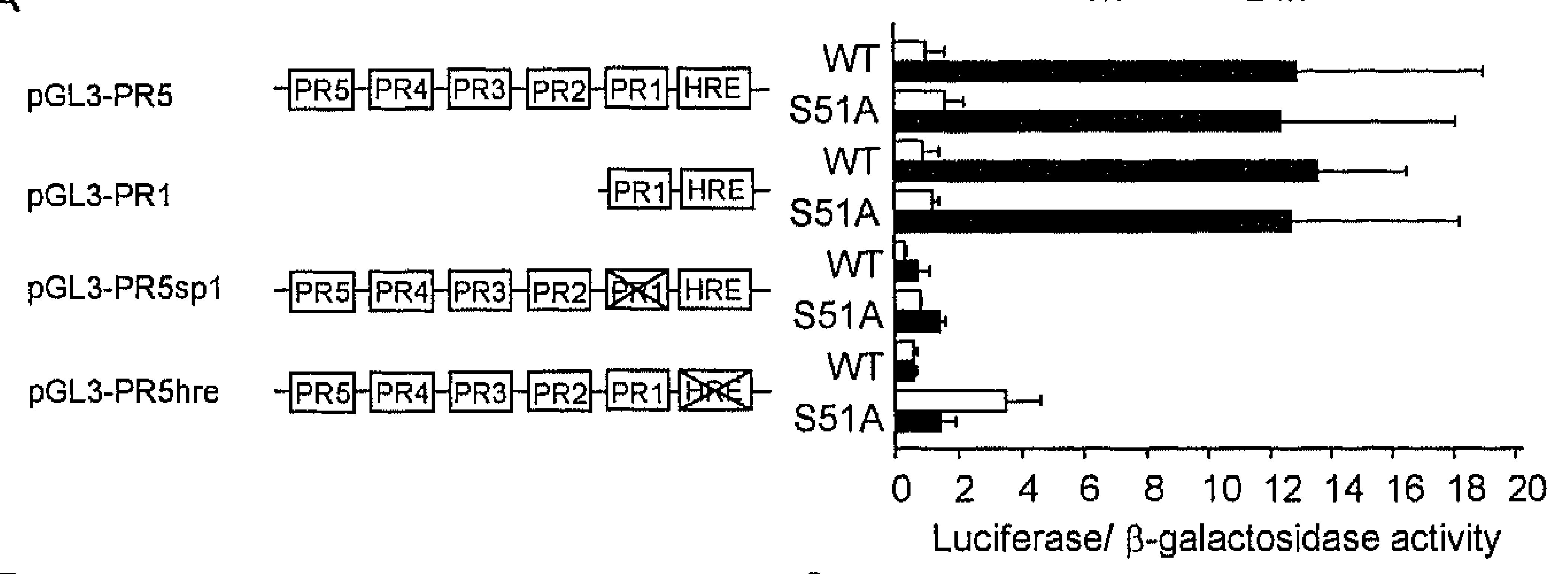

B

C
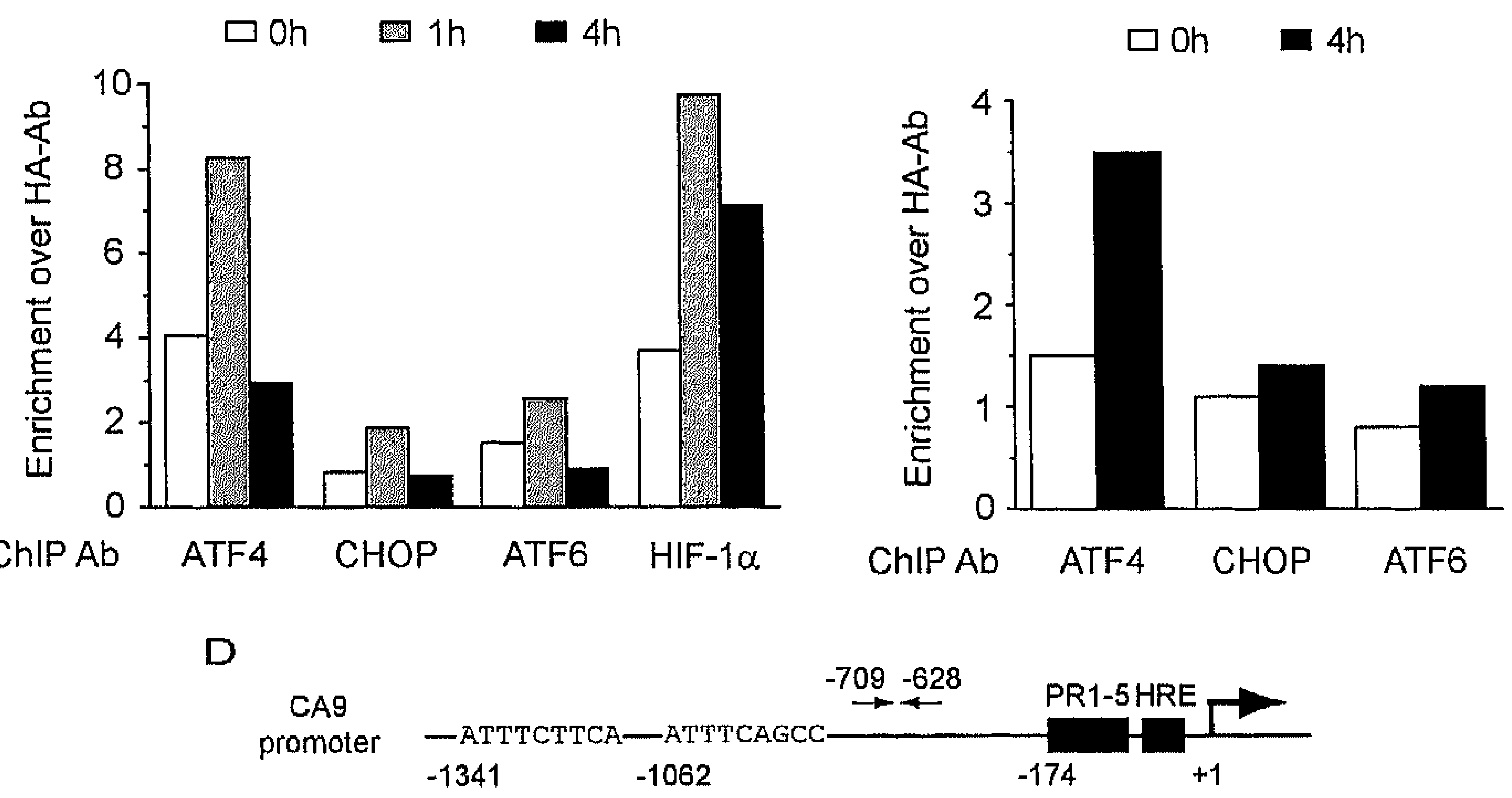

E

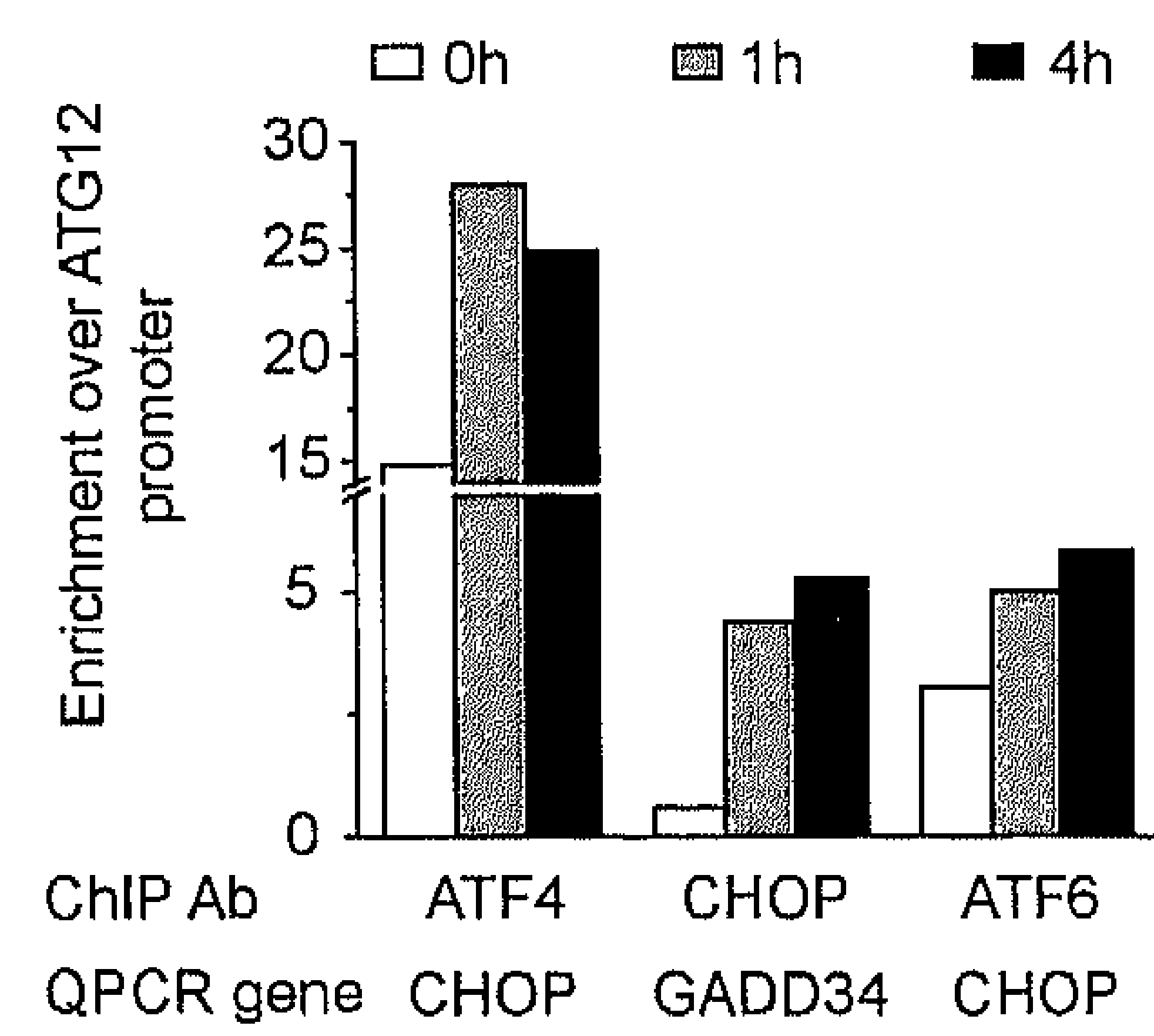

F

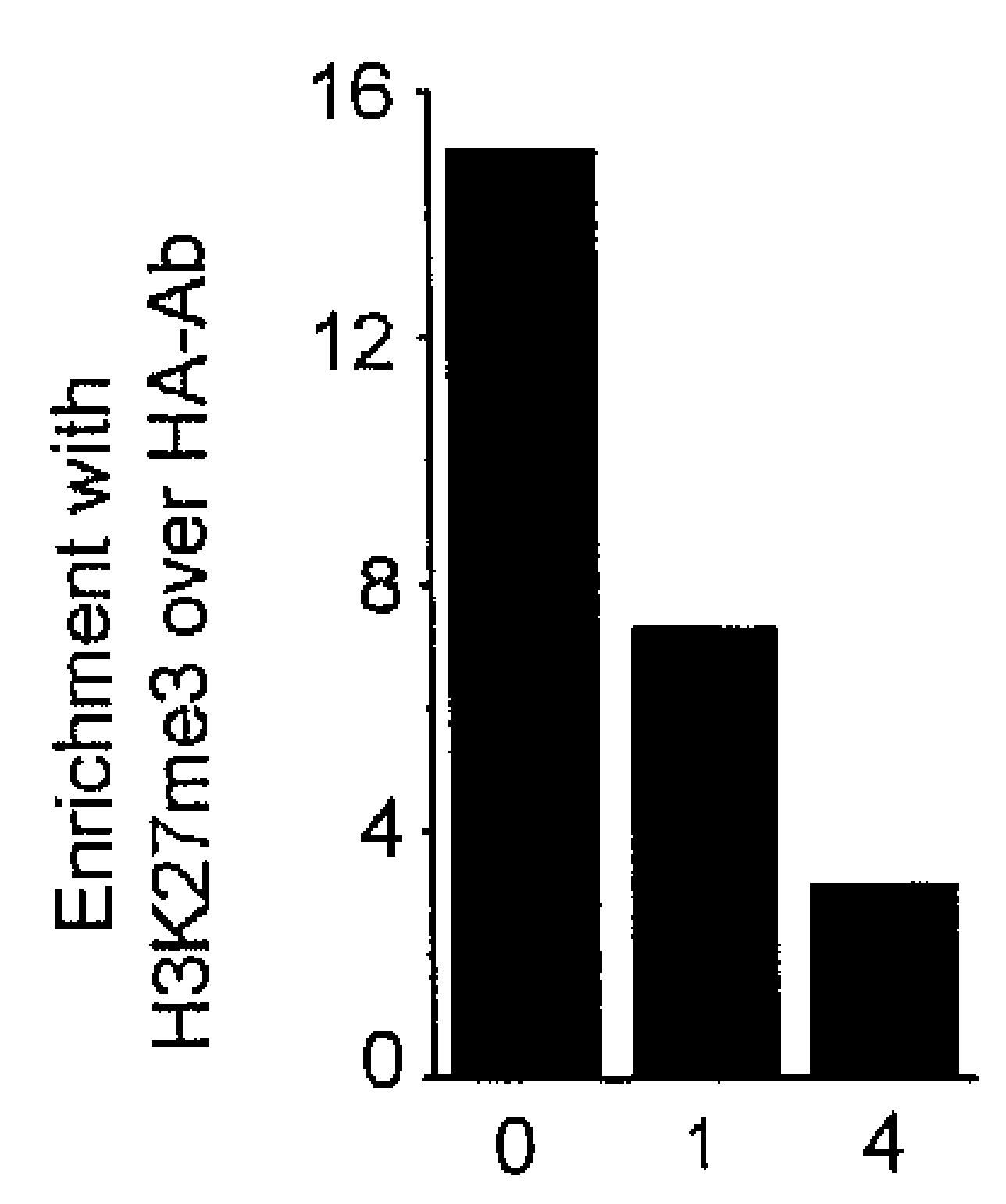

Hypoxic time (hrs) 
Figure 5. ATF4 binds to the CA9 promoter region. (A) WT CA9 promoter construct or mutant versions with PR2-5 deleted, sp1 binding site or HRE inactivated were transiently cotransfected with pCDNA3-LacZ into WT MEF or S51A MEF cells. Luciferase activity was measured relative to $\beta$-galactosidase expression after 24 hrs of hypoxia after which fold induction was calculated for each construct. Bars represent the average of 4 independent experiments plus standard deviation. ChIP analysis was performed on U373 (B) and HCT116 (C) cells after exposure to normoxia or hypoxia using indicated antibodies. Q-PCR was used to determine fold enrichment of the CA9 promoter region over that obtained with negative control antibody HA. (D) Localization of putative ATF4 binding sites in CA9 promoter region. (E) Positive controls for ChIP analysis. Fold enrichment of $\mathrm{CHOP}$ and GADD34 promoter regions for ATF4/ ATF6 and CHOP respectively over unrelated promoter region of ATG12 gene. (F) ChIP analysis using U373 cells with antibodies against H3K27me during hypoxia, fold enrichment over negative control anti-HA Ab.

To investigate the functional importance of ATF4 in regulating CA9 expression during hypoxia we knocked down ATF4 using siRNA. In addition we knocked down the ATF4 regulated transcription factor $\mathrm{CHOP}$ as well as HIF-1 $\alpha$ and ATF6 as positive and negative controls respectively. We achieved $50-90 \%$ knock-down of our target genes compared to scrambled (SCR) siRNA control in U373 (Figure 6A) and HCT116 cells (supplementary figure 3A). U373 cells depleted from HIF- $1 \alpha$ or ATF4 exhibited a strong reduction in CA9 mRNA compared with the SCR controls after $16 \mathrm{hrs}$ of hypoxia (Figure 6B). Depletion of the other UPR related transcription factors CHOP and ATF6 did not affect CA9 induction during hypoxia.

To determine if cellular levels of ATF4 were directly influencing endogenous CA9 expression we also over-expressed ATF4 in U373 cells and exposed them to hypoxic conditions. Again basal mRNA levels of CA9 were unaffected under aerobic conditions (data not shown), however after 16 hours of hypoxia CA9 mRNA levels were approximately 2-fold higher compared to cells bearing an empty vector control (Figure 6C). Taken together, our data support a model in which hypoxic induction of CA9 is dependent on the PERK controlled arm of the UPR involving direct binding of ATF4 to the CA9 promoter. 


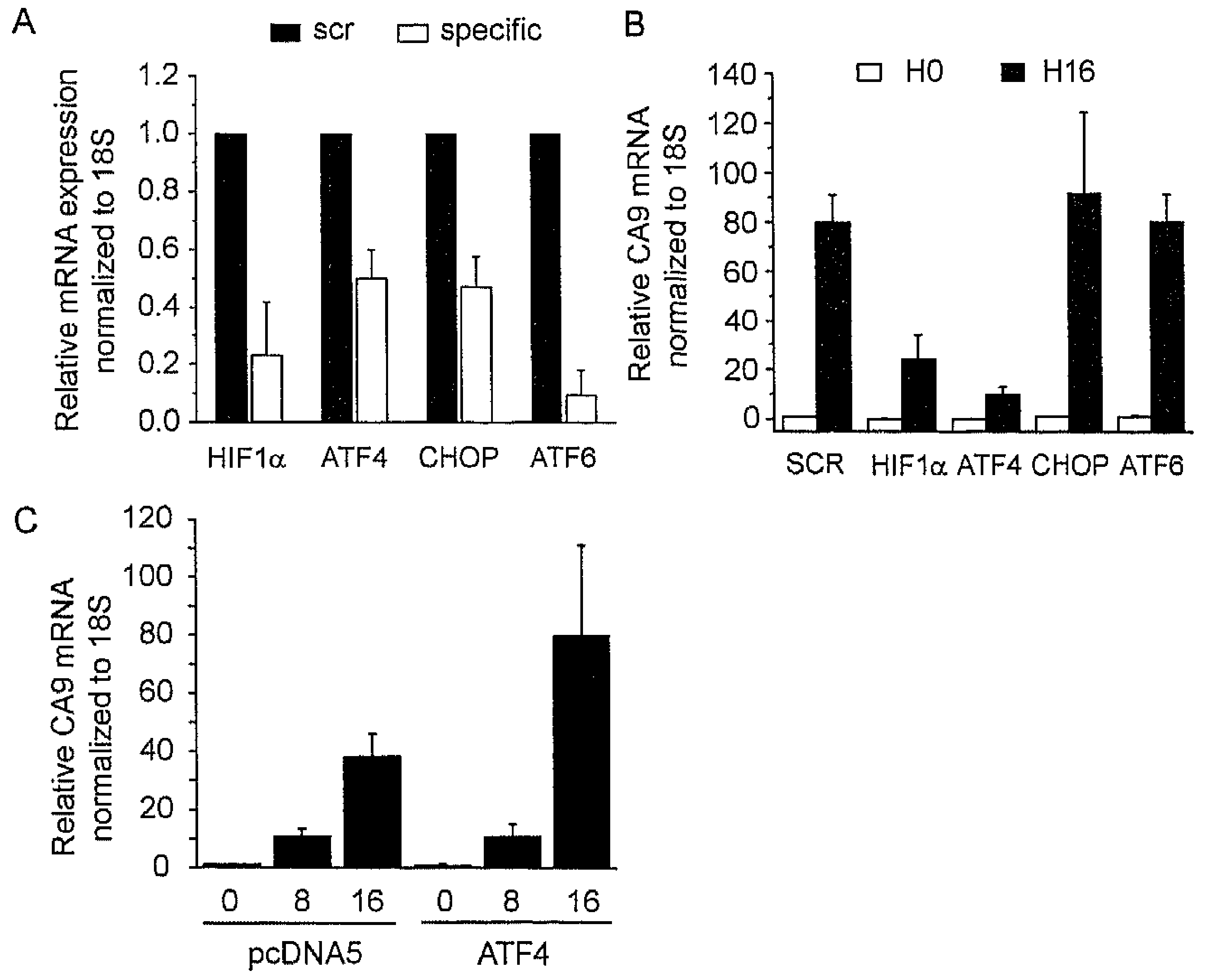

Figure 6. ATF4 is required for CA9 mRNA expression during hypoxia. U373 cells were transiently transfected with siRNA duplexes directed against HIF1 $\alpha$, ATF4, CHOP and ATF6. Twenty four hrs post transfection cells were exposed to 16 hrs of hypoxia. (A) The knockdown efficiency was determined for gene specific siRNAs using Q-PCR. Expression was normalized to mRNA levels in cells transfected with scrambled control. (B) Relative expression of CA9 mRNA post transfection with siRNA after culturing under normoxia or 16 hours of hypoxia. (C) Exogenous ATF4 was over expressed in U373 cells and exposed to 0,8 and 16 hours of hypoxia. Relative levels of CA9 mRNA were determined using Q-PCR compared to empty vector control under aerobic conditions.

\section{The UPR mediates expression of CA9 in vivo}

Finally to investigate the role of the UPR on induction of CA9 expression in vivo, xenograft tumors were grown from isogenic U373 cells bearing empty vector, eIF2 $\alpha$ S51A or shRNA against HIF-1 $\alpha$ as a positive control. Immunohistochemical staining using an antibody against CA9 revealed an 
overall decreased staining intensity in the HIF-1 $1 \alpha$ knock-down tumor sections compared to the empty vector controls (Figure $7 A+B$ ). Tumors derived from the U373 cells with defective eIF2 $\alpha$ phosphorylation also showed a reduction in CA9 levels that was comparable to the HIF-1 $\alpha$ knock-down tumors (Figure $7 \mathrm{C}$ ).
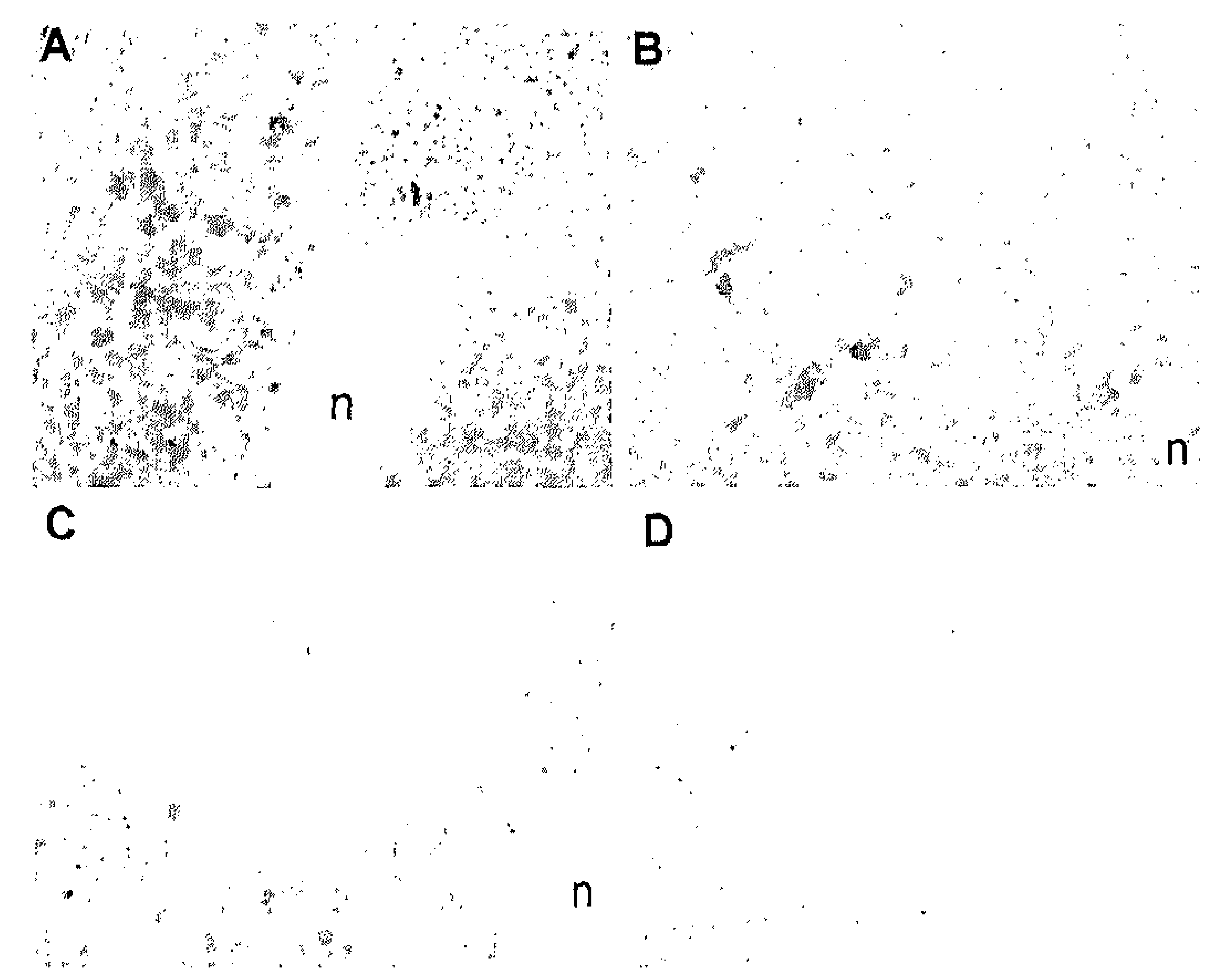

Figure 7. Overall CA9 expression is reduced in section of U373 S51A xenograft tumors. Immunohistochemical analysis of CA9 expression in xenograft tumors derived from U373 cells bearing empty vector, eIF2 $\alpha$ S51A or shRNA against HIF1a. Sections were stained with anti-CA9 polycional antibody (A) U373 pCDNA, (B) U373 HIF-1 $\alpha$ knock-down, (C) U373 S51A and (D) negative control. Staining is predominantly membranous and focused to perinecrotic regions in all tumors. $n$, regions of necrosis. 


\section{Discussion}

It is well established that hypoxic conditions induce CA9 transcription through the transcription factor HIF, which binds to the HRE element in the CA9 promoter ${ }^{5}$. Here we showed that in addition to HIF, PERK signaling to eIF $2 \alpha$ is necessary for CA9 induction during hypoxia. This was demonstrated using several different genetic models in both human and mouse cells. The requirement for UPR signaling during hypoxia does not extend to CA9 induced by high cell density under aerobic conditions ${ }^{33}$, which has been shown to involve the PI3K pathway ${ }^{16}$ and MAPK signaling ${ }^{17}$. These results support the existence of two distinct mechanisms for CA9 transcriptional regulation, a HIF dependent mechanism activated under low oxygen levels and a PI3K/ MAPK dependent pathway at high cell density. Here we provide evidence that the former mechanism relies on simultaneous PERK/eIF2 $\alpha$ signaling.

The dependence of CA9 expression on eIF $2 \alpha$ phosphorylation is directly mediated by ATF4. Knockdown or overexpression of ATF4 decreased or increased CA9 expression during hypoxia respectively. Furthermore, we showed that ATF4 directly binds to the CA9 promoter. Interestingly published microarray data from several studies has shown that CA6 is also responsive to UPR activation ${ }^{30}, 34,35$. In contrast to CA9 this effect is dependent on the ATF4 responsive transcription factor CHOP and not directly through ATF $4^{30}$, 35. Nontheless these data suggest an important and broader role for PERK/eIF2 $\alpha /$ ATF4 in regulating $\mathrm{pH}$ levels through up regulation of carbonic anhydrases.

The function of ATF4 on the CA9 promoter and its potential COoperation with HIF1 is not fully understood. Induction of the UPR under non-hypoxic conditions using DTT or tunicamycin was unable to induce CA9. Interestingly we found that the CA9 promoter contains trimethylation marks on lysine residue 27 of histone $\mathrm{H} 3$ under aerobic conditions. These marks are generally correlated to a transcriptional repressive state $^{32}$. We found that this repressive mark was lost after exposure to hypoxia for periods as short as 1 hour. These data suggest 
that hypoxia influences CA9 expression in part through an epigenetic mechanism. This result is interesting in relation to a recent report suggesting that hypoxia may affect global changes in histone methylation ${ }^{36}$. To what extent this is dependent on ATF4 expression remains unclear.

ATF4 may also influence CA9 through its known interaction with the transcriptional co-activator $\mathrm{p} 300 / \mathrm{CBP}^{37}$ or $\mathrm{p} 300 / \mathrm{CBP}$-associated factor $(\mathrm{PCAF})^{38}$. Both $\mathrm{p} 300 / \mathrm{CBP}$ and PCAF contain histone acetyltransferase (HAT) activity and function as co-activators in the transcriptional regulation of many genes. Our data indicate that recruitment of HIF during hypoxia is essential but not sufficient for complete transcriptional activation of CA9. Since $\mathrm{p} 300 / \mathrm{CBP}$ is required for HIF mediated transcription it is attractive to suggest a model in which ATF4 and HIF cooperate through their ability to recruit p300/CBP and/or PCAF. Such cooperation would be meaningful in certain hypoxic range, which simultaneously activates both pathways.

Adaptation to hypoxic conditions through activation of the UPR is an important survival mechanism for tumor cells. The Ire1/Xbp1 and PERK/eIF $2 \alpha$ arms of the UPR have been shown to play a critical role in determining hypoxia sensitivity ${ }^{20}{ }^{39}$. PERK knockout MEFs have decreased hypoxia tolerance and tumor growth in xenograft studies due to their inability to activate eIF $2 \alpha$ phosphorylation ${ }^{20}$. It is tempting to speculate that this growth defect could be due in part to decreased CA9 expression. It has previously been shown that knock-down of CA9 in MDA468 and MDA231 breast carcinoma cell lines using siRNA resulted in a significant reduction in clonogenic survival after hypoxic exposure compared to control cells ${ }^{14}$. However whether decreased CA9 expression is responsible for the decrease growth rate observed with the eIF $2 \alpha$ deficient tumors needs further investigation. PERK/eIF2 $\alpha$ phosphorylation and upregulation of ATF4 is also involved in other important processes including angiogenesis ${ }^{34}$, and maintenance of energy homeostasis ${ }^{40}$. Thus, as is the case for HIF, the contribution of the UPR to hypoxia tolerance is likely to 
be multi-factorial. Our data also contribute to a growing overlap in the contribution of UPR and HIF dependent responses to the hypoxic tumor phenotype. $\mathrm{pH}$ regulation, angiogenesis, and energy homeostasis are also all strongly regulated by $\mathrm{HIF}^{3}$. In this regard it is important to note that the oxygen dependecies of HIF activation and the UPR are not the same. Whereas HIF is activated at relatively moderate hypoxic conditions (1$2 \%$ ), full activation of the UPR requires more severe hypoxia ${ }^{19,}{ }^{41}$. Consequently the UPR and HIF pathways may allow cells to adjust the strength of these responses as a function of oxygen concentration.

CA9 has become widely adopted as a surrogate marker of tumor hypoxia used to classify patients that likely respond poorly to therapy ${ }^{42,43}$. Although CA9 expression correlates well with exogenous marker of hypoxia pimonidazole its correlation with HIF-1 $\alpha$ is much weaker ${ }^{44,45}$. CA9 expression is often detected in the perinecrotic regions of solid tumors where HIF1a levels are reduced ${ }^{45}$. Several possible explanations have been suggested for this poor co-localization ${ }^{45}$ including the large difference in protein half-life between HIF-1 $\alpha$ and CA9. Due to its long half-life CA9 expression may be present in areas that have been reperfused with oxygen and no longer express HIF-1a. Our results provide an additional mechanism to explain high CA9 expression in perinecrotic tumor areas. Tumor cells in these regions are likely to exhibit activation of PERK due to the extremely hypoxic nature of these regions. Therefore simultaneous activation of the UPR and HIF, might drive higher CA9 expression in these areas. The requirement of UPR signaling may also explain why some HIF-1 positive tumors fail to express CA9.

\section{Acknowledgements}

We would like to thank Natasja Lieuwes for technical assistance. This work was financially supported by Dutch Science Organization (ZonMW-NWO Top grant 912-03-047 to BW, 908-02-040 \& 016.046.362 to JWV), the Dutch Cancer Society (KWF grant UM 2003-2821 to BW), and the EU $6^{\text {th }}$ 
The UPR contributes to hypoxia-induced expression of CA9

framework program (Euroxy program to BW), ZonMW VENI Grants 016.056 .015 to $M K$ and 916.76 .158 to $K R$.

\section{References}

1. Brown JM, Wilson WR: Exploiting tumour hypoxia in cancer treatment, Nat Rev Cancer 2004, 4:437-447

2. Hockel M, Vaupel P: Biological consequences of tumor hypoxia, Semin Oncol 2001, 28:36-41.

3. Semenza GL: Targeting HIF-1 for cancer therapy, Nat Rev Cancer 2003, 3:721-732

4. Maxwell PH, Wiesener MS, Chang GW, Clifford SC, Vaux EC, Cockman ME, Wykoff CC, Pugh CW, Maher ER, Ratcliffe PJ: The tumour suppressor protein VHL targets hypoxia-inducible factors for oxygen-dependent proteolysis, Nature 1999, 399:271-275

5. Wykoff CC, Beasley NJ, Watson PH, Turner KJ, Pastorek J, Sibtain A, Wilson GD, Turley $H$, Talks KL, Maxwell PH, Pugh CW, Ratcliffe PJ, Harris AL: Hypoxia-inducible expression of tumor-associated carbonic anhydrases, Cancer Res 2000, 60:7075-7083

6. Pastorekova S, Parkkila $S$, Zavada J: Tumor-associated carbonic anhydrases and their clinical significance, Adv Clin Chem 2006, 42:167-216

7. Potter CP, Harris AL: Diagnostic, prognostic and therapeutic implications of carbonic anhydrases in cancer, $\mathrm{Br}$ ] Cancer 2003, 89:2-7

8. Svastova E, Hulikova A, Rafajova M, Zat'ovicova M, Gibadulinova A, Casini A, Cecchi A, Scozzafava A, Supuran CT, Pastorek J, Pastorekova S: Hypoxia activates the capacity of tumor-associated carbonic anhydrase IX to acidify extracellular pH, FEBS Lett 2004, 577:439-445

9. Martinez-Zaguilan R, Seftor EA, Seftor RE, Chu YW, Gillies RJ, Hendrix MJ: Acidic $\mathrm{pH}$ enhances the invasive behavior of human melanoma cells, Clin Exp Metastasis 1996, 14:176-186

10. Moellering RE, Black KC, Krishnamurty $C$, Baggett BK, Stafford $P$, Rain $M$, Gatenby RA, Gillies RJ: Acid treatment of melanoma cells selects for invasive phenotypes, Clin Exp Metastasis 2008,

11. Stubbs M, McSheehy PM, Griffiths JR, Bashford CL: Causes and consequences of tumour acidity and implications for treatment, Mol Med Today 2000, 6:1519

12. Supuran CT: Carbonic anhydrases: novel therapeutic applications for inhibitors and activators, Nat Rev Drug Discov 2008, 7:168-181

13. Dubois L, Douma K, Supuran CT, Chiu RK, van Zandvoort MA, Pastorekova $S$, Scozzafava A, Wouters BG, Lambin P: Imaging the hypoxia surrogate marker CA IX requires expression and catalytic activity for binding fluorescent sulfonamide inhibitors, Radiother Oncol 2007, 83:367-373

14. Robertson N, Potter C, Harris AL: Role of carbonic anhydrase IX in human tumor cell growth, survival, and invasion, Cancer Res 2004, 64:6160-6165

15. Kaluz S, Kaluzova M, Opavsky R, Pastorekova S, Gibadulonova A, Dequiedt $F$, Kettmann R, Pastorek J: Transcriptional regulation of the MN/CA9 gene coding for the tumor-associated carbonic anhydrase IX., JBC 1999, 274:3258832595

16. Kaluz S, Kaluzova M, Chrastina A, Olive PL, Pastorekova S, Pastorek J, Lerman MI, Stanbridge EJ: Lowered oxygen tension induces expression of the hypoxia marker MN/carbonic anhydrase IX in the absence of hypoxia-inducible 
factor 1 alpha stabilization: a role for phosphatidylinositol 3'-kinase, Cancer Res 2002, 62:4469-4477

17. Kopacek J, Barathova M, Dequiedt F, Sepelakova J, Kettmann R, Pastorek J, Pastorekova S: MAPK pathway contributes to density- and hypoxia-induced expression of the tumor-associated carbonic anhydrase IX, Biochim Biophys Acta 2005, 1729:41-49

18. Kaluz S, Kaluzova M, Stanbridge EJ: The role of extracellular signal-regulated protein kinase in transcriptional regulation of the hypoxia marker carbonic anhydrase IX, J Cell Biochem 2006, 97:207-216

19. Koumenis C, Wouters BG: "Translating" tumor hypoxia: unfolded protein response (UPR)-dependent and UPR-independent pathways, Mol Cancer Res 2006, 4:423-436

20. Bi M, Naczki C, Koritzinsky M, Fels D, Blais J, Hu N, Harding H, Novoa I, Varia M, Raleigh J, Scheuner D, Kaufman RJ, Bell J, Ron D, Wouters BG, Koumenis $C$ : ER stress-regulated translation increases tolerance to extreme hypoxia and promotes tumor growth, Embo ] 2005, 24:3470-3481

21. Feldman $D E$, Chauhan V, Koong AC: The Unfolded Protein Response: A Novel Component of the Hypoxic Stress Response in Tumors, Mol Cancer Res 2005, 3:597-605

22. Koritzinsky M, Magagnin MG, van den Beucken $T$, Seigneuric $R$, Savelkouls $K$, Dostie J, Pyronnet S, Kaufman RJ, Weppler SA, Voncken JW, Lambin P, Koumenis C, Sonenberg N, Wouters BG: Gene expression during acute and prolonged hypoxia is regulated by distinct mechanisms of translational control, Embo J 2006, 25:1114-1125

23. Blais JD, Filipenko V, Bi M, Harding HP, Ron D, Koumenis C, Wouters BG, Bell JC: Activating transcription factor 4 is translationally regulated by hypoxic stress, Mol Cell Biol 2004, 24:7469-7482

24. van den Beucken $T$, Koritzinsky $M$, Wouters BG: Translational control of gene expression during hypoxia, Cancer Biol Ther 2006, 5:749-755

25. Scheuner D, Song B, McEwen E, Liu C, Laybutt R, Gillespie P, Saunders T, Bonner-Weir $S$, Kaufman RJ: Translational control is required for the unfolded protein response and in vivo glucose homeostasis, Mol Cell 2001, 7:11651176.

26. Pastorekova S, Zavadova Z, Kostal M, Babusikova O, Zavada J: A novel quasiviral agent, MaTu, is a two-component system, Virology 1992, 187:620-626

27. Bracken AP, Pasini D, Capra M, Prosperini E, Colli E, Helin K: EZH2 is downstream of the PRB-E2F pathway, essential for proliferation and amplified in cancer, Embo J 2003, 22:5323-5335

28. Donze $O$, Jagus $R$, Koromilas AE, Hershey JW, Sonenberg N: Abrogation of translation initiation factor elF-2 phosphorylation causes malignant transformation of NIH 3T3 cells, Embo J 1995, 14:3828-3834

29. Harding HP, Novoa I, Zhang Y, Zeng H, Wek R, Schapira M, Ron D: Regulated translation initiation controls stress-induced gene expression in mammalian cells, Mol Cell 2000, 6:1099-1108

30. Sok J, Wang X-Z, Batchvarova $N$, Kuroda $M$, Harding $H$, Ron D: CHOPDependent Stress-Inducible Expression of a Novel Form of Carbonic Anhydrase VI, Mol. Cell. Biol. 1999, 19:495-504

31. Fawcett TW, Martindale JL, Guyton KZ, Hai T, Holbrook NJ: Complexes containing activating transcription factor (ATF)/CAMP-responsive-elementbinding protein (CREB) interact with the CCAAT/enhancer-binding protein (C/EBP)-ATF composite site to regulate Gadd153 expression during the stress response, Biochem J 1999, 339 (Pt 1):135-141 
32. Bernstein BE, Meissner A, Lander ES: The mammalian epigenome, Cell 2007, 128:669-681

33. Lieskovska J, Opavsky R, Zacikova L, Glasova M, Pastorek J, Pastorekova S: Study of in vitro conditions modulating expression of MN/CA IX protein in human cell lines derived from cervical carcinoma, Neoplasma 1999, 46:17-24

34. Blais JD, Addison $C L$, Edge $R$, Falls $T$, Zhao $H$, Wary $K$, Koumenis $C$, Harding HP, Ron D, Holcik M, Bell JC: Perk-dependent translational regulation promotes tumor cell adaptation and angiogenesis in response to hypoxic stress., Mol. Cell. Biol. 2006, 26:9517-9532

35. Marciniak SJ, Yun CY, Oyadomari S, Novoa I, Zhang $Y$, Jungreis R, Kazuhiro Nagata $K$, Heather P. Harding HP, Ron D: CHOP induces death by promoting protein synthesis and oxidation in the stressed endoplasmic reticulum, Genes \& Dev. 2004 2004, 18:3066-3077

36. Johnson $A B$, Denko N, Barton $M C$ : Hypoxia induces a novel signature of chromatin modifications and global repression of transcription, Mutat Res 2008, 640:174-179

37. Liang G, Hai T: Characterization of human activating transcription factor 4 , a transcriptional activator that interacts with multiple domains of CAMPresponsive element-binding protein (CREB)-binding protein, $]$ Biol Chem 1997, 272:24088-24095

38. Cherasse $Y$, Maurin AC, Chaveroux C, Jousse C, Carraro V, Parry L, Deval C, Chambon C, Fafournoux P, Bruhat A: The p300/CBP-associated factor (PCAF) is a cofactor of ATF4 for amino acid-regulated transcription of $\mathrm{CHOP}$, Nucleic Acids Res 2007, 35:5954-5965

39. Romero-Ramirez $L$, Cao $H$, Nelson $D$, Hammond $E$, Lee A-H, Yoshida $H$, Mori K, Glimcher LH, Denko NC, Giaccia AJ, Le Q-T, Koong AC: XBP1 Is Essential for Survival under Hypoxic Conditions and Is Required for Tumor Growth, Cancer Res 2004, 64:5943-5947

40. Hochachka PW, Buck LT, Doll CJ, Land SC: Unifying theory of hypoxia tolerance: molecular/metabolic defense and rescue mechanisms for surviving oxygen lack, Proc Natl Acad Sci U S A 1996, 93:9493-9498

41. Magagnin MG, Koritzinsky $M$, Wouters BG: Patterns of tumor oxygenation and their influence on the cellular hypoxic response and hypoxia-directed therapies, Drug Resist Updat 2006, 9:185-197

42. Airley RE, Loncaster J, Raleigh JA, Harris AL, Davidson SE, Hunter RD, West CM, Stratford IJ: GLUT-1 and CAIX as intrinsic markers of hypoxia in carcinoma of the cervix: relationship to pimonidazole binding, Int J Cancer 2003, 104:85-91

43. Beasley NJ, Wykoff CC, Watson PH, al. e: Carbonic anhydrase IX, an endogenous hypoxia marker, expression in head and neck squamous cell carcinoma and its relationship to hypoxia, necrosis and microvessel density., Cancer Res 2001, 61:5262-5267

44. Jankovic B, Aquino-Parsons $\mathrm{C}$, Raleigh JA, Stanbridge EJ, Durand RE, Banath JP, MacPhail SH, Olive PL: Comparison between pimonidazole binding, oxygen electrode measurements, and expression of endogenous hypoxia markers in cancer of the uterine cervix, Cytometry Part B: Clinical Cytometry 2006, 70B:45-55

45. Sobhanifar S, Aquino-Parsons C, Stanbridge EJ, Olive P: Reduced Expression of Hypoxia-Inducible Factor- 1 \{alpha\} in Perinecrotic Regions of Solid Tumors, Cancer Res 2005, 65:7259-7266 


\section{CHAPTER 8}

\section{Targeting translational control pathways, an in vitro study}

In preparation

Twan van den Beucken ${ }^{1}$, Kasper M.A. Rouschop ${ }^{1}$, Kim Savelkouls, Philippe Lambin, Marianne Koritzinsky and Bradly G. Wouters.

${ }^{1}$ contributed equally to this study 


\title{
Chapter 8
}

\begin{abstract}
Hypoxia is a common feature of solid tumors and is associated with an aggressive phenotype, resistance to therapy and poor prognosis. These adverse affects are mediated by changes in protein expression that occur at both the transcriptional and translational level through several oxygensensitive pathways. Here we report the development of an isogenic tumor model in which three of these pathways can be conditionally regulated. Inhibition of the PERK/ eIF $2 \alpha$ pathway of the unfolded protein response (UPR) was achieved by overexpression of eIF $2 \alpha$ S51A, GADD34 or shRNA against PERK. Likewise HIF signaling was targeted through shRNA against HIF- $1 \alpha$ and mTOR signaling by shRNA against TSC1 or overexpression of eIF4E. All cell lines were validated using Q-PCR or Western blot analysis. Using these cell lines we demonstrated that HIF signaling is important for cell growth under hypoxic conditions as HIF-1a knockdown cells exhibit 3fold reduced proliferation rates. Conversely, all pathways independently contribute to hypoxia tolerance since their disruption significantly sensitized cells to hypoxia induced cell death. Finally we showed that expression of this isogenic inducible system can be used efficiently in established xenograft tumors by administration of doxycline to the drinking water of tumor bearing mice. This will allow us in the future to determine the relative importance of targeting these pathways in vivo in pre-established tumors in a manner that closely mimics the therapeutic situation.
\end{abstract}




\section{Introduction}

The majority of solid human tumors contain areas that are poorly oxygenated ${ }^{1}$. It has been demonstrated that tumor hypoxia causes treatment resistance through induction of angiogenesis, metastasis and selection for cells with reduced apoptotic potential ${ }^{2-4}$. Together these studies indicate that hypoxia promotes a more malignant tumor phenotype which is of high clinical relevance 5 . The negative impact on patient prognosis is caused in part by the activation of several oxygen sensitive signaling pathways. These hypoxia response response pathways have therefore received widespread attention and are being evaluated as potential targets for the treatment of human tumors ${ }^{6}$. The best understood of these pathways involves the stabilization and activation of the HIF family of transcription factors. Their activation during hypoxia mediates the induction of more than 60 genes affecting important pathways like glycolysis and angiogenesis ${ }^{2}$.

Work from our lab and others has demonstrated that global mRNA translation is inhibited during hypoxia ${ }^{7-9}$. This inhibition contributes to maintaining cellular energy homeostasis, but is not secondary to energy depletion as it occurs very rapidly. Moreover, it has a major affect on hypoxia induced gene expression by modulating gene specific translation and thus on the hypoxic cellular phenotype ${ }^{7,10} 11$. Hypoxia causes an overall inhibition of mRNA translation through at least two different signal transduction pathways. The first response is mediated rapidly during acute hypoxia through PERK-dependent phosphorylation of eukaryotic initiation factor alpha (eIF2 $\alpha$ ). This prevents the formation of a functional ternary complex and thus translation initiation. Data suggest that this type of translation inhibition is transient, with significant recovery during continued exposure to hypoxia ${ }^{7}, 12$. However, translation rates remain inhibited due to the activation of a second response mediated via disruption of the eIF4F complex which is essential for cap-dependent translation. Hypoxia prevents eIF4E: eIF4G interaction and thus functional 


\section{Chapter 8}

eIF4F through increased eIF4E:4E-T and eIFE:4E-BP1 complex formation" 12,13 .

It has been shown that the cells capacity to mediate translational control through eIF2 $\alpha$ is important for their survival in response to hypoxia, and for promoting tumor growth or response to treatment ${ }^{9,14}$. This has generated attention for translational control pathways in addition to HIF as potential targets for tackling hypoxia in cancer treatment. However, the majority of these results have used mouse embryo fibroblasts from knockout or knockin mice whose applicability to epithelial human cancers is questionable. It also remains unclear as to the relative importance of these pathways in contributing to tumor growth, metastasis, or response to therapy. This is further complicated by the tumor microenviroment which harbors areas of acute, chronic or cycling levels of hypoxia, causing a range of different oxygen tensions ${ }^{15,} 16$ that differentially affect these various oxygen sensitive pathways.

In this study, we have developed and tested a new set of tumor cell lines to investigate the relative importance of multiple adaptive mechanisms during hypoxia. We describe a novel inducible isogenic cell model in which different hypoxia responsive pathways can be either activated or inactivated by administration of tetracycline or doxycycline. We propose that this model system can provide valuable information about the importance of these hypoxia response pathways in established tumors and their potential for targeting in combination with other therapies.

\section{Material and Methods}

\section{Tissue culture conditions}

Exponentially growing human colon cancer cell line HCT116 and human glioblastoma cell line U373 were grown in DMEM and MEM alpha medium respectively supplemented with $10 \%$ foetal calf serum (FCS) (SigmaAldrich). For hypoxic exposure cells were transferred to a hypoxic culture chamber (MACS VA500 microaerophilic workstation, Don Whitley 
Scientific, Shipley, UK). The atmosphere in the chamber consisted of $5 \%$ $\mathrm{H}_{2}, 5 \% \mathrm{CO}_{2}$, the desired concentration of $\mathrm{O}_{2}$ and residual $\mathrm{N}_{2}$. An anoxic atmosphere was ensured by the inclusion of a catalyst in the hypoxic chamber that catalysed the conversion of $\mathrm{H}_{2}$ with any $\mathrm{O}_{2}$ to $\mathrm{H}_{2} \mathrm{O}$.

\section{Generation isogenic Flp-in expressing cells}

Stable isogenic U373 and HCT116 cell lines expressing a single Flp-in recombination site were generated using the Flp-in ${ }^{T M}$ T-Rex system (Invitrogen) according to the manufacturers' instructions. Briefly, the Flpin recombination plasmid PFRT/LacZeo was transfected into U373 and HCT116 using lipofectamine as described by the manufacturer (Invitrogen). Stable $\beta$-galacosidase positive clones were obtained after selection zeocin rich media. Next the tet-repressor was introduced into these clones by transfection with PCDNA6/TR and selection for blastocidin resistance. Clones were selected that only contained 1 Flp-in recombination site. Proliferation under aerobic and hypoxic conditions of the final clones as well as their hypoxia sensitivity was found to be exactly as the parental cell line.

\section{Western blotting}

Cells were lysed directly in hot $\left(95^{\circ} \mathrm{C}\right)$ sample buffer $(125 \mathrm{mM}$ Tris-HCl, $5 \%$ glycerol, $0.04 \%$ sodium dodecyl sulphate and $1 \mathrm{M} \beta$-mercaptoethanol), resolved by SDS-PAGE and blotted onto polyvinylidene fluoride (PVDF) membranes. Blots were probed with antibodies directed against phospho(serine 51)-eIF2 $\alpha$ (Research Genetics), PERK (Santa Cruz), eIF4E and TSC1 (Cell signaling). Bound antibodies were visualized using HRPlinked secondary antibodies (anti-rabbit (Cell Signaling Technologies) and anti-mouse (Sigma)) and $E C L$ luminescence (Pierce). Exposure to $1 \mathrm{mM}$ DTT for 1 hour was used as a positive control for eIF $2 \alpha$ phosphorylation. This strongly reducing agent causes protein misfolding in the endoplasmic reticulum which activates the eIF2 $\alpha$ kinase PERK. 


\section{Chapter 8}

\section{Growth and survival}

Cell number was counted before $\left(\mathrm{N}_{0}\right)$ and after $(\mathrm{N})$ hypoxic exposure. Doubling time $T$ after growth time $t$ was calculated from the formula $\mathrm{N}=\mathrm{N}_{0} 2^{(\mathrm{t} / \mathrm{T})}$. For clonogenic survival, single cells were plated and exposed to hypoxic conditions for the indicated time period. Colonies containing more than 50 cells were scored as a surviving cell. Surviving fraction was calculated as the ratio between surviving cells and cells plated, corrected for plating efficiency (PE), i.e. survival in untreated controls.

\section{Tumor xenograft growth}

Animal experiments were performed using adult NMRI (nu/ nu) female mice $(28-32 \mathrm{~g})$ from the animal facility of the Catholic University of Leuven in Belgium. The animal facilities and experiments were in accordance with local institutional guidelines for animal welfare and were approved by the Animal Ethics Committee of the university. Three million U373 and U373-eGFP cells were resuspended in $100 \mu$ growth medium and injected sub-cutaneously in to the lateral flank of recipient mice. Tumors were grown until they reached $300 \mathrm{~mm}^{3}$ after which the mice were left untreated or received $2 \mathrm{~g} / \mathrm{L}$ doxycicline in their drinking water. Tumors were excised after 4 days and fixed in 1-4 \% formaldehyde. GFP expression was assessed using the Bonsai imaging apparatus (Siemens Germany).

\section{Results}

\section{Development of an isogenic inducible cell model}

The objective of this study was to compare the relative importance of several hypoxia responsive pathways on cellular phenotype during this stress. For this purpose we created a new tumor cell model based on the commercial Flp-in ${ }^{T M}$ T-Rex system (Invitrogen). In this system, creation of an acceptor cell line results in the ability to easily introduce a number of different transgenes or shRNAs into a single genomic site through a flp mediated recombination event. The cell lines created from this acceptor 
cell line are truly isogenic containing only a single integration at a common genomic site. In addition, the system we engineered is under the control of the tetracycline responsive promoter and can thus expression of the inserted gene or shRNA is dependent on the addition of tetracycline.

We successfully generated U373 and HCT116 cell lines that contain a Flp-in recombination site at a single location in the chromosome (see materials and methods). We introduced an ORF coding for enhanced green fluorescent protein (eGFP) in these two cell lines to validate inducebility and expression levels. Figure $1 \mathrm{~A}$ shows that eGFP expression is readily visible after addition of $1 \mu \mathrm{g} / \mathrm{ml}$ tetracycline for $24 \mathrm{~h}$. The inducebility was assessed in more detail using FACS analysis. Within $4 \mathrm{hrs}$ after addition of tetracycline eGFP levels were more than 50-fold elevated and reach maximum levels after $24 \mathrm{hrs}$ (Figure 1B). The expression was furthermore not compromised under hypoxic conditions. Similar data were observed for HCT116 cells expressing eGFP (data not shown).

A

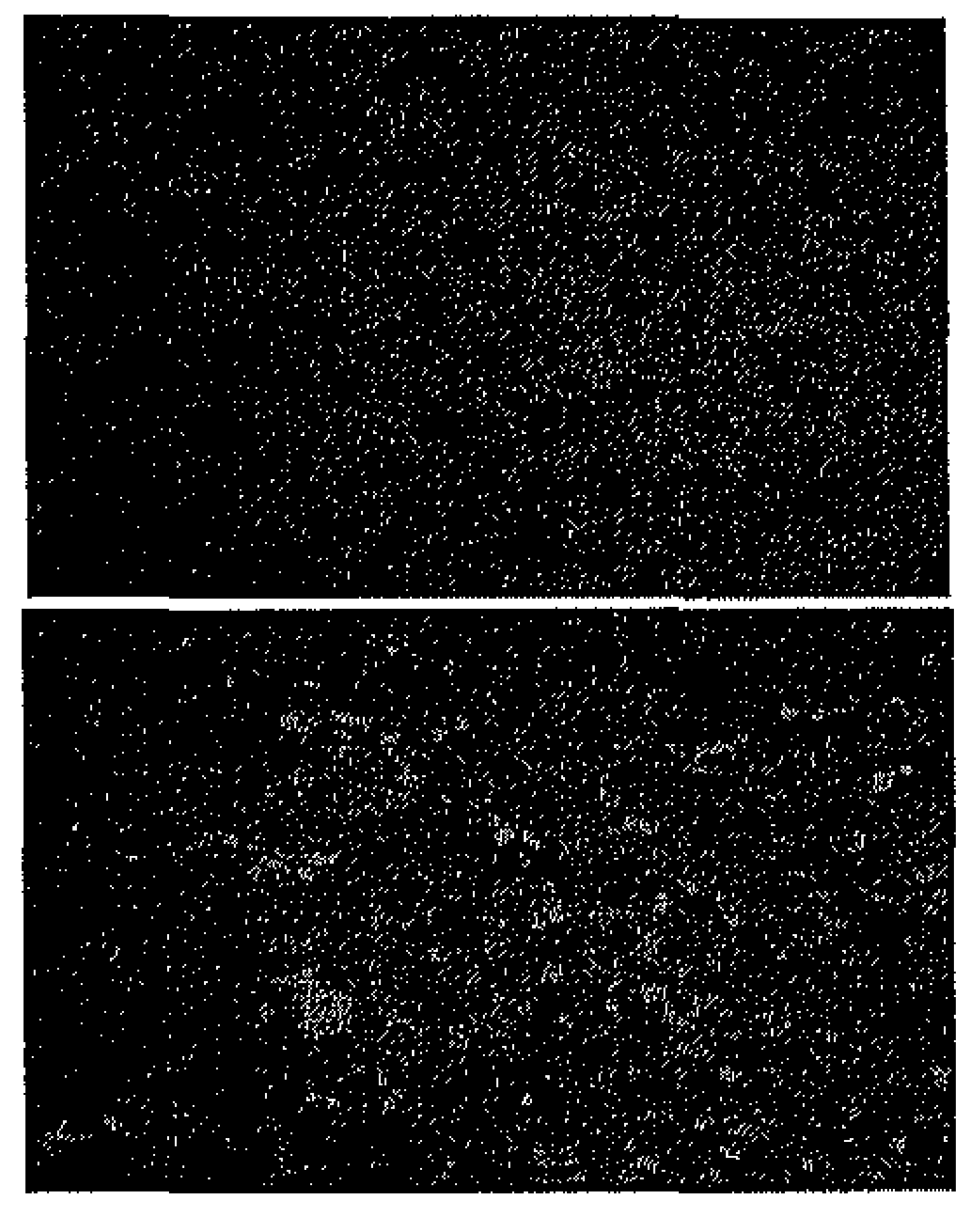

B

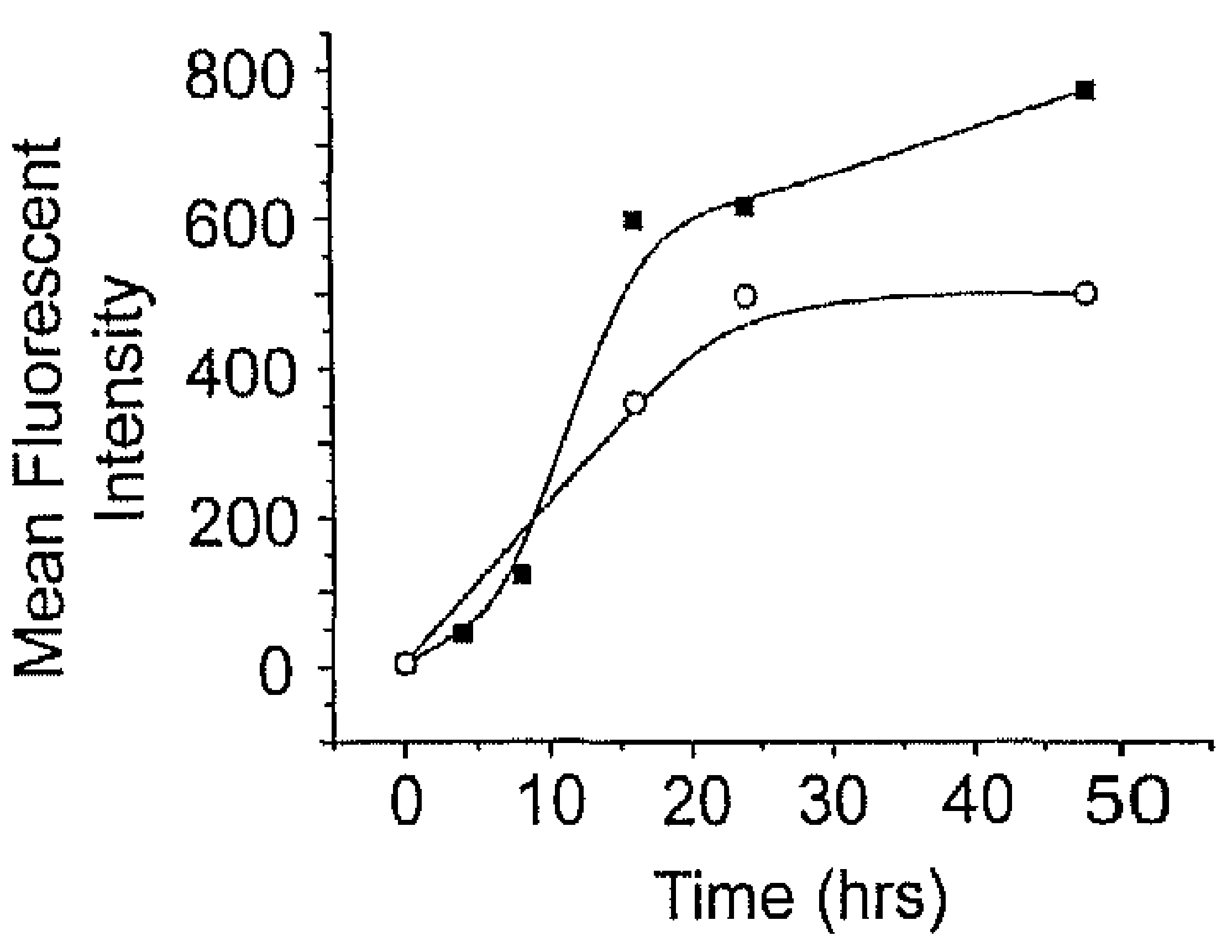

Figure 1. Validation of isogenic model system using eGFP. A. U373 cells stably expressing eGFP from an inducible integrated construct were left untreated (top panel) or exposed to $1 \mu \mathrm{g} / \mathrm{ml}$ tetracycline for $24 \mathrm{hrs}$ (bottom panel). B. Quantitative FACS analysis of GFP expression after addition of tetracycline under aerobic (filled boxes) and hypoxic (open circles) conditions. 


\section{Modulation of hypoxia responsive pathways}

The low basal and high inducability observed following introduction of eGFP indicated that the acceptor lines would be suitable for use in developing cell lines targeting hypoxia responsive pathways. We used multiple approaches to disable oxygen sensing through each of the three main hypoxia response pathways that affect transcription (HIF) and translation (PERK/eIF2 $\alpha$ and eIF4F) (see figure 2A).

A

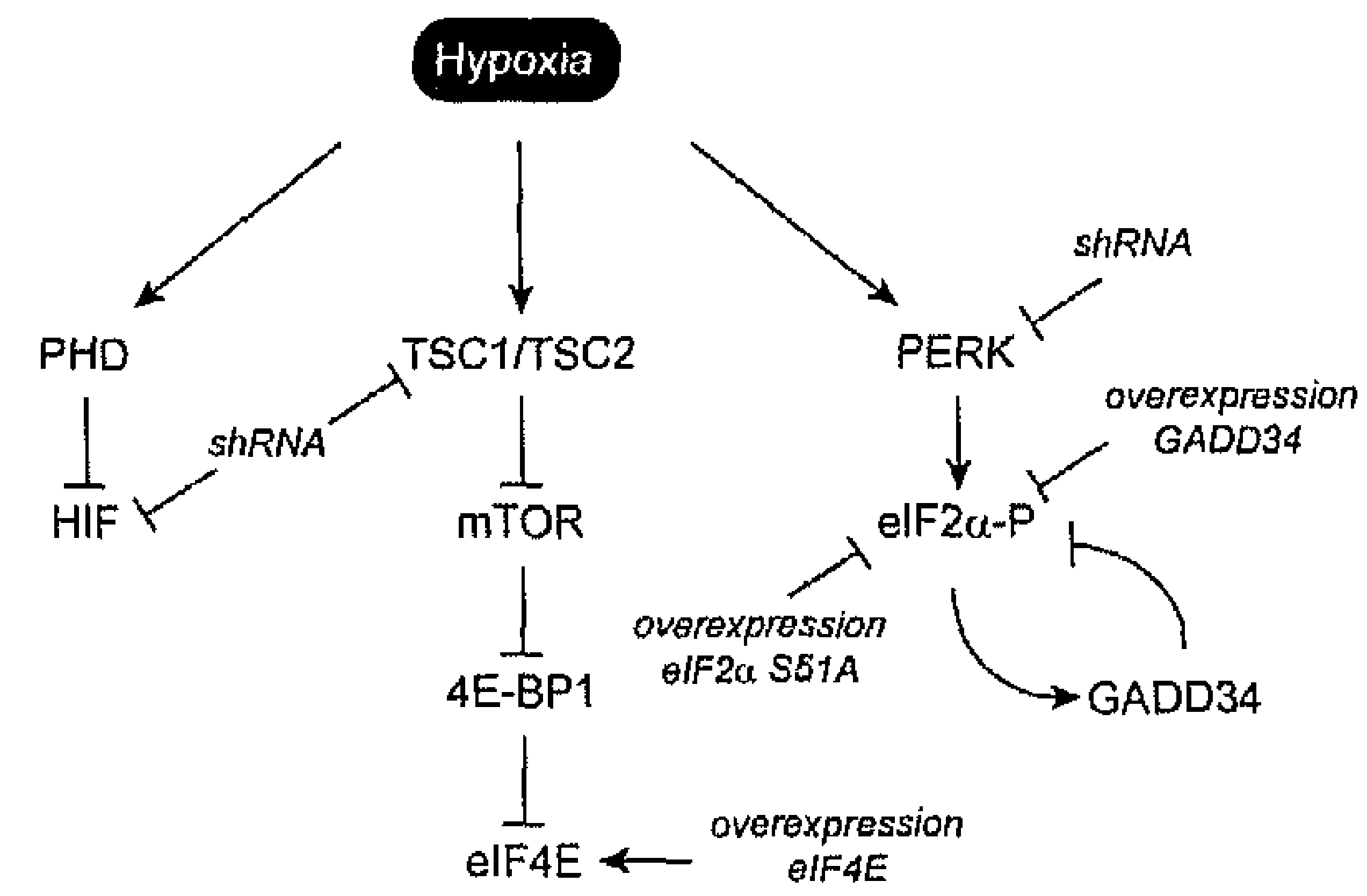

B

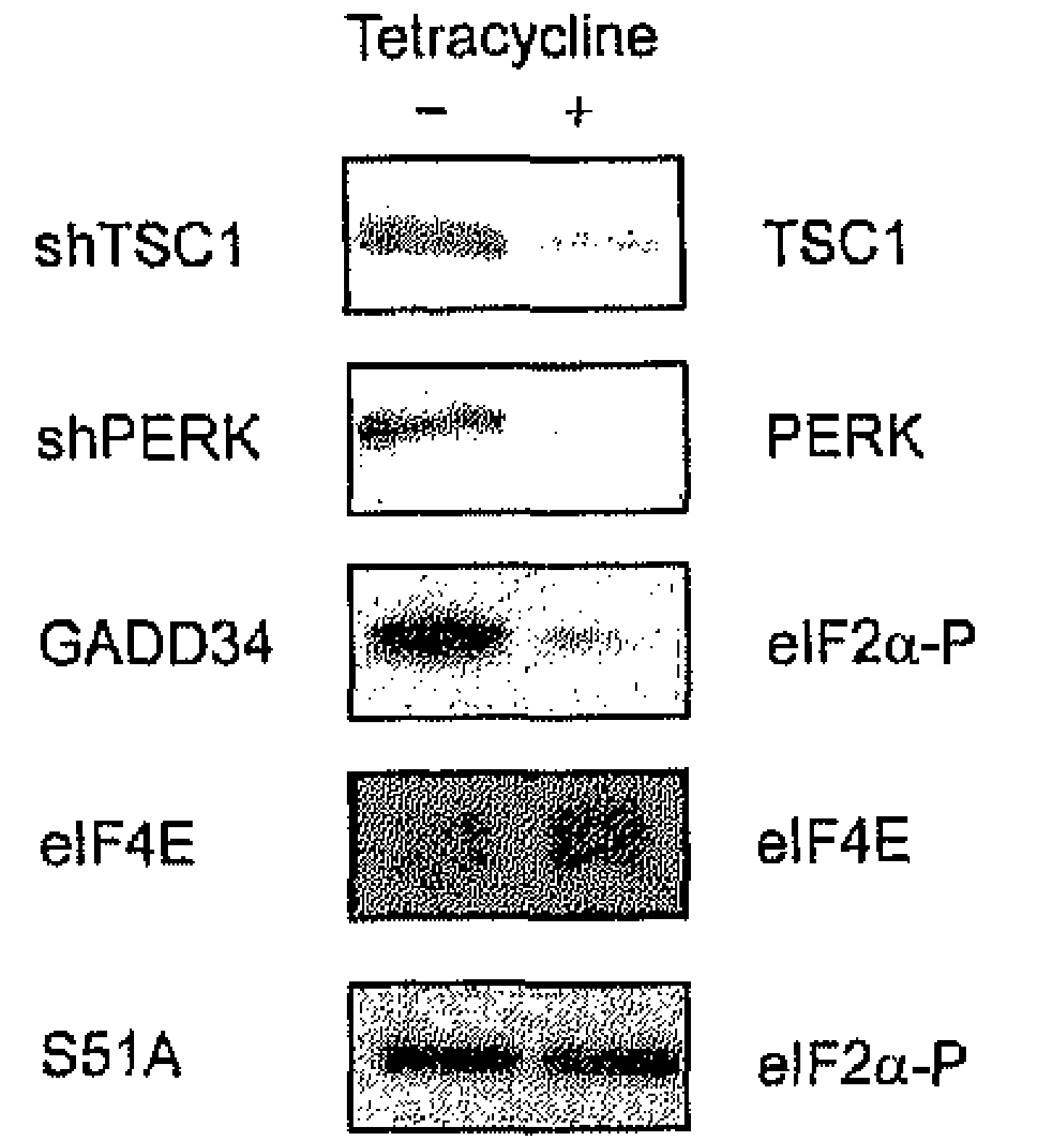

C

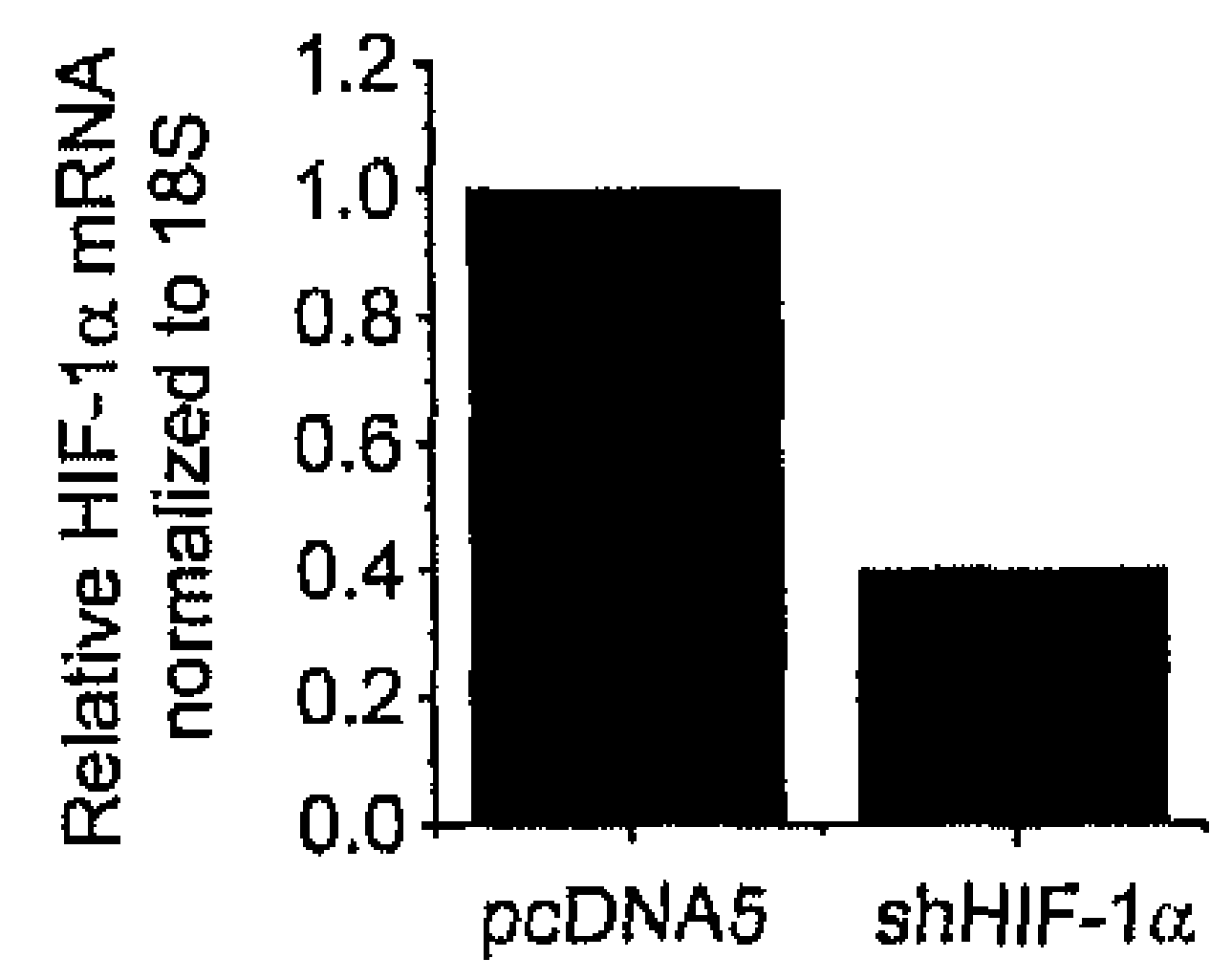

Figure 2. Interference with hypoxia activated response pathways. (A) Deactivation of these pathways is achieved either through expression of shRNA, dominant negative (DN) or wild type versions of the indicated target molecules. (B) Western blot analysis of generated cell lines during hypoxia. Expressed constructs

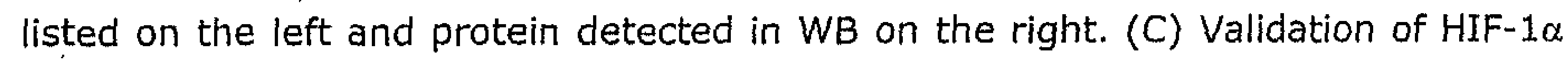
knockdown cells was done using Q-PCR. 
For this purpose we targeted the PERK/eIF2 $\alpha$ pathway at 3 different levels, overexpressing GADD34, dominant negative eIF2 $\alpha$ S51A, or short hairpin interfering RNA (shRNA) against PERK (shPERK). GADD34 expression leads to the activation of a phosphatase that dephosphorylates eIF $2 \alpha$ and thus prevents hypoxic inactivation of this protein. The HIF response was disrupted by shRNA against HIF-1a. Signaling through eIF4F during hypoxia was disrupted by targeting either mTOR activation (using a shRNA against TSC1) or by overexpression of eIF4E. For validation of the different constructs cells were cultured for 48 hours in the presence of tetracycline followed by 4 hours of hypoxic (Figure $2 \mathrm{~B}+\mathrm{C}$ ) or aerobic (data not shown) conditions. Subsequently, the expression levels of the inserted transgenes or their targets were evaluated. shRNA was effective at silencing TSC1, PERK and HIF1 $\alpha$. Probing the phosphorylation of eIF2 $\alpha$ indicated that overexpression of GADD34 was effective. Expression of the eIF2 $\alpha$ S51A allele has no major effect on endogenous eIF $2 \alpha$ phosphorylation levels (Figure $2 \mathrm{C}$ ). However it significantly rescues the translational repression during hypoxia compared to control cells as determined by polysomal RNA fractionation (data not shown).

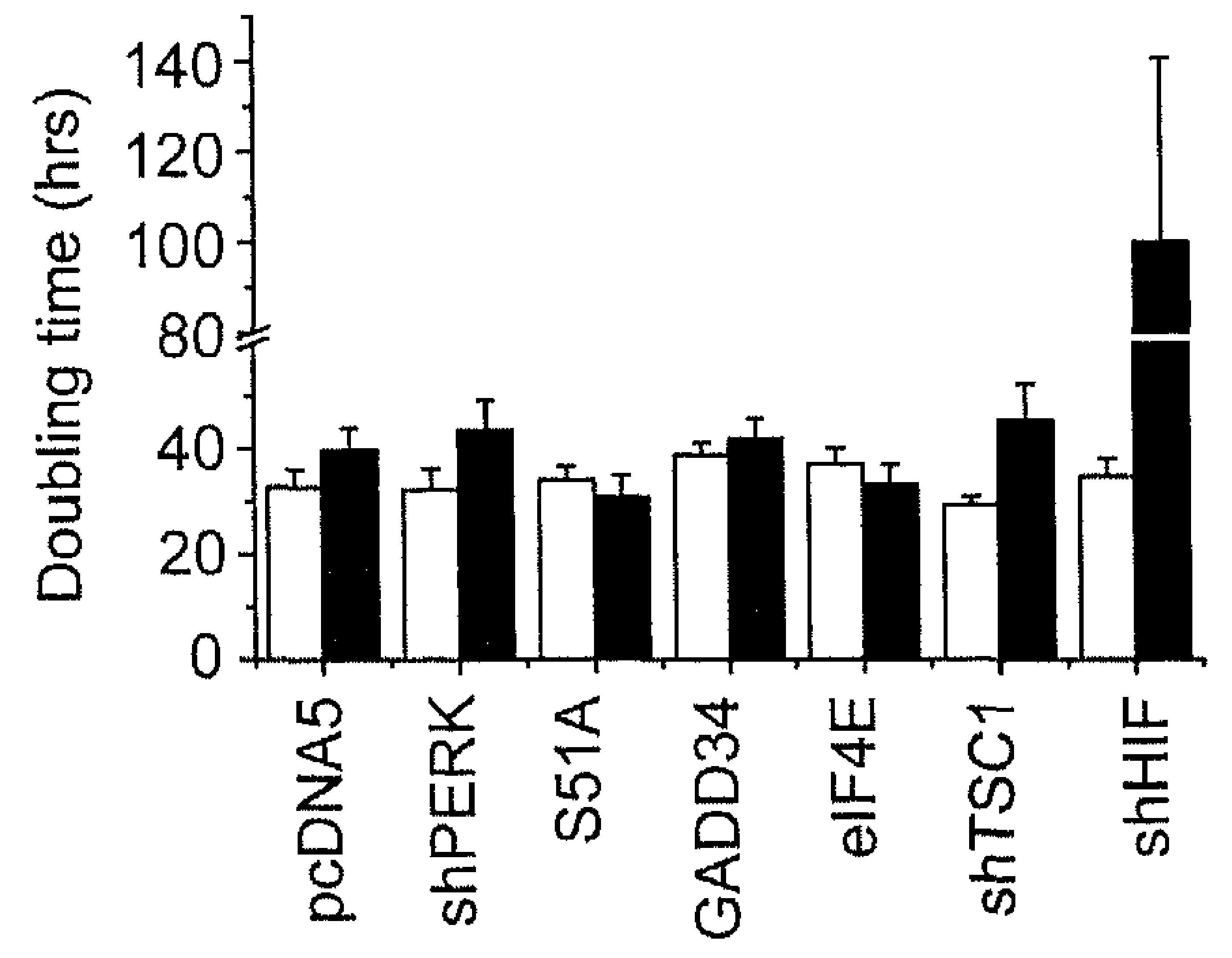

Figure 4. Effect of disrupted hypoxia response pathways on proliferation. HCT116 cells defective in indicated pathways were grown under $0.2 \%$ oxygen. Subsequently fold increase in doubling time $(T)$ was calculated. 


\section{Chapter 8}

A
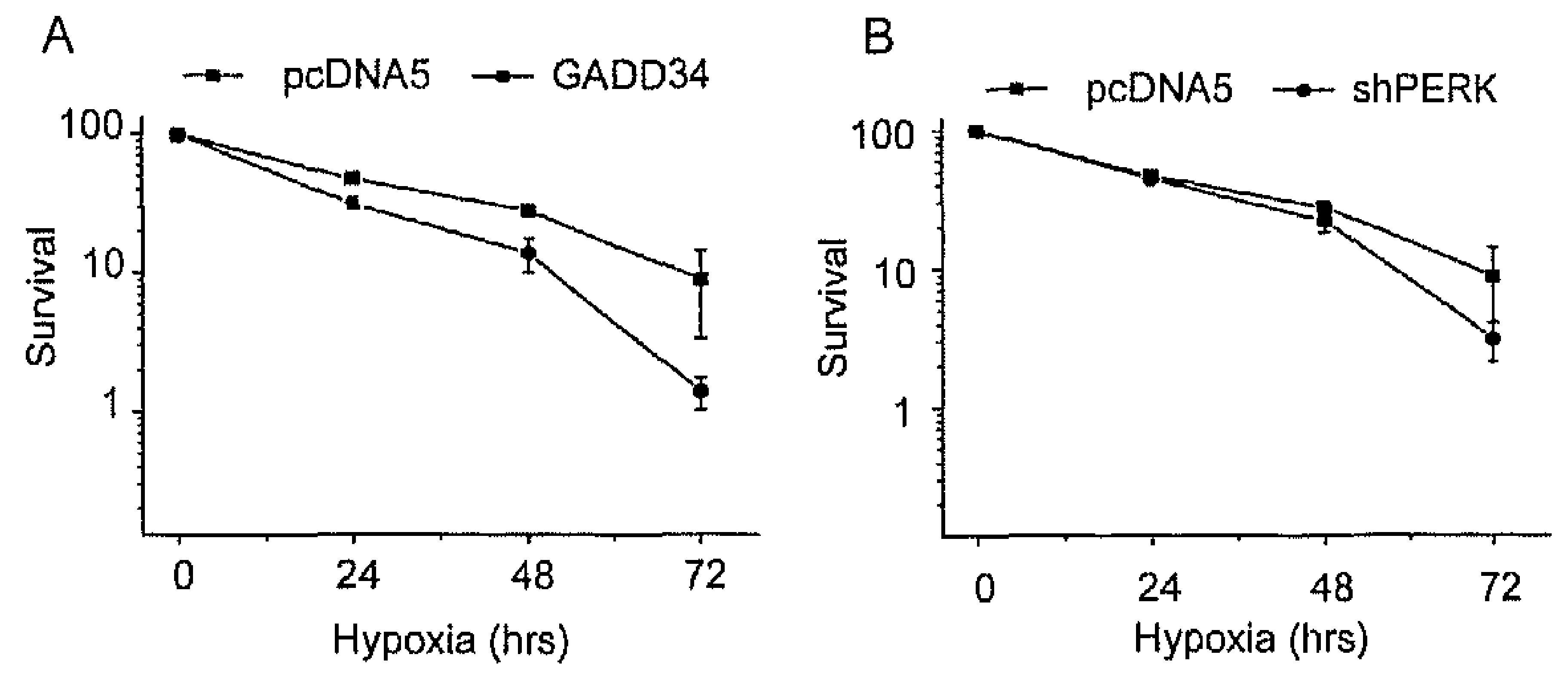

C
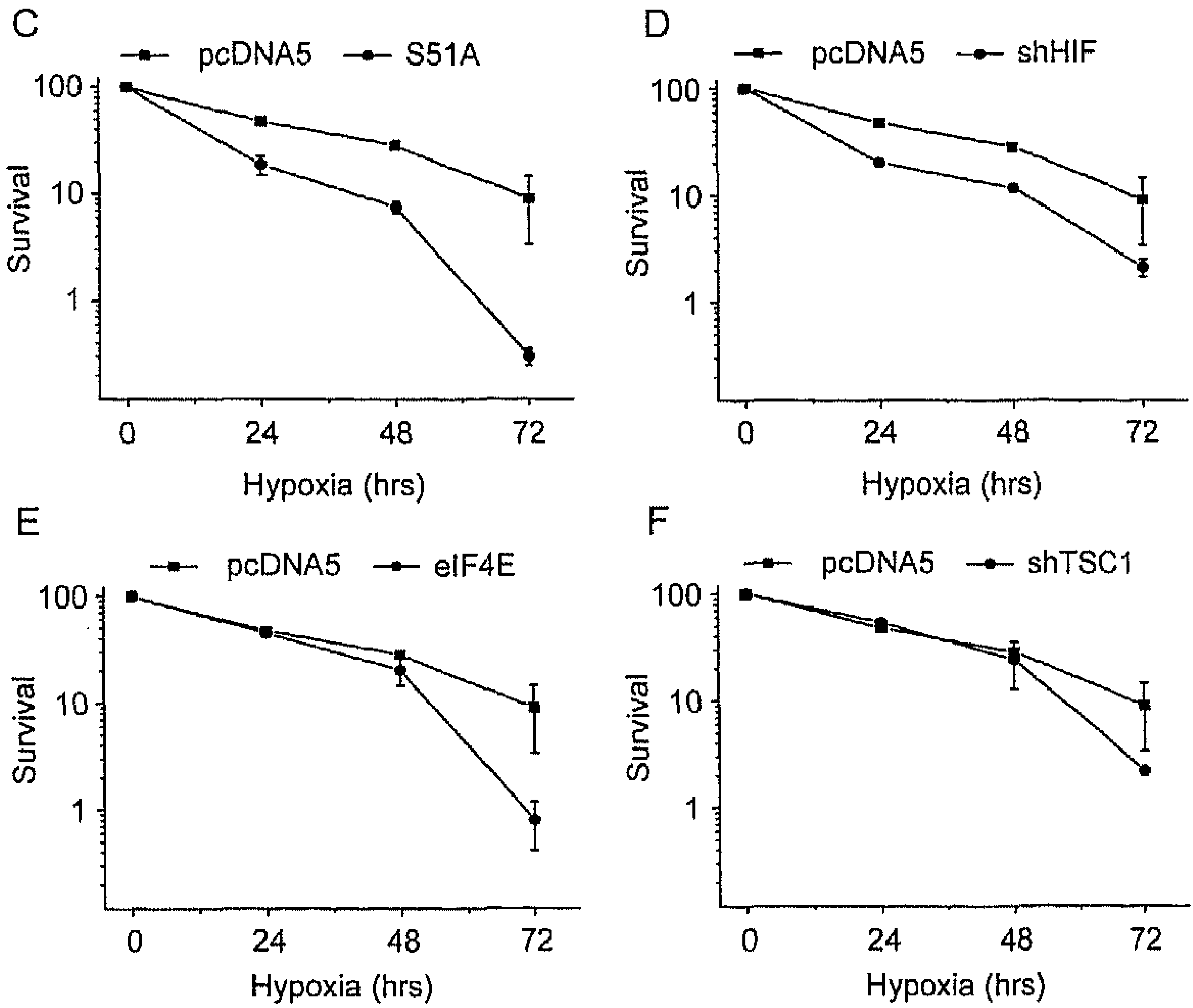

Figure 5. Relative importance of hypoxia response pathways on hypoxia sensitivity. HCT116 cells defective in indicated pathways were seeded at low concentrations and exposed to anoxic conditions $\left(>0.02 \% \mathrm{O}_{2}\right)$ for the indicated period of time. Clonogenic survival was determined after $\sim 14$ days.

Next we investigated whether interference with any of these mechanisms might lead to differences in proliferation rates under aerobic or hypoxic conditions. For this purpose, HCT116 cells were treated with tetracycline for 48 hrs and subsequently grown for different periods under 
either aerobic or hypoxic conditions. As expected, we observed that interference with any of these pathways had no influence on cell growth under optimal aerobic conditions (Figure 4). Interestingly this is also true for culture during hypoxia for most of the tested cell lines except for the HIF knock down cells. These cells exhibit a $\sim 3$-fold reduction in proliferation rate upon hypoxia.

The ability to activate the PERK/ eIF $2 \alpha$ pathway in response to hypoxia has previously been shown to be vital for cells to tolerate hypoxic stress ${ }^{9}$, 14. However the relative importance of PERK/ eIF $2 \alpha$ activation compared to the other two pathways is currently unknown. Therefore we investigated this by determining clonogenic cell survival after exposure to severe and moderate hypoxic conditions. We were able to confirm the importance of PERK/ eIF2 $\alpha$ activation during hypoxia for cell survival. Preventing eIF2 $\alpha$ phosphorylation significantly sensitized HCT116 cells to severe hypoxia (Figure $5 A-C$ ). This was observed with all constructs that prevent eIF $2 \alpha$ phosphorylation, although overexpression of eIF $2 \alpha$ S51A was most potent in sensitizing cells to hypoxia induced cell death. Knockdown of HIF-1a revealed a similar effect on clonogenic survival as the PERK/eIF2 $\alpha$ pathway (Figure 5D). For both pathways this effect was apparent after 24 hours of hypoxia. In contrast, interference with mTOR signaling on hypoxia tolerance achieved through overexpression of eIF4E or knockdown of TSC1 became evident after 72 hours of exposure to hypoxia (Figure $5 \mathrm{E}, \mathrm{F}$ ). Exposure to more moderate hypoxia $\left(0.2 \% \mathrm{O}_{2}\right)$ was found to delay induction of cell death by about 24 hours, bud did not alter the relative sensitivity of any individual cell line (data not shown).

Finally we investigated whether we could use this system is to inducibly regulate expression of a transgene within a pre-established tumor. To obtain proof of principle we implanted U373-eGFP cells sub-cutaneously into the flank of nude mice. Expression of GFP was induced when the tumor size reached $300 \mathrm{~mm}^{3}$ by addition of doxycycline to the drinking water of the mice. After 4 days fluorescence images were taken ex vivo. Significant induction of eGFP was visible in doxycycline treated U373-eGPF 


\section{Chapter 8}

tumors (Figure 6), although some 'leaky' GFP expression was observed in untreated tumors. Nevertheless these data demonstrate that expression of desired transgenes can be induced in established xenograft tumors.

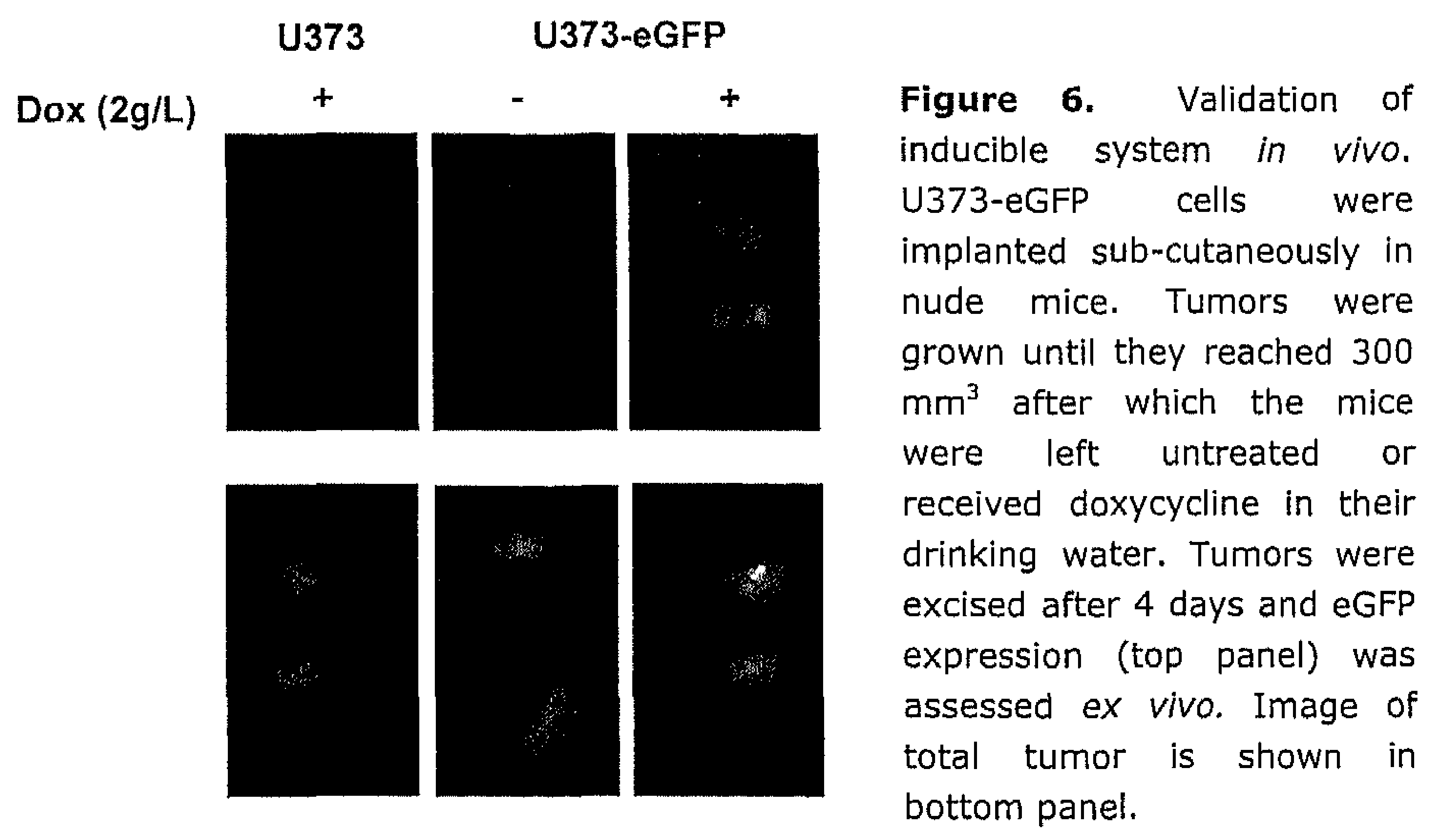

$0.5 \mathrm{~s}$

\section{Discussion}

Tumor cells adapt to hypoxic conditions by activating molecular response pathways that include those regulating both MRNA translation (PERK/ eIF $2 \alpha$ and mTOR/eIF4F) and transcription (HIF). Failure to execute this adaptive response program sensitizes tumor cells to hypoxia induced cell death. These pathways therefore provide interesting targets for the development of novel cancer therapies.

In this study we report the development of a novel tumor model designed to investigate the relative importance of different hypoxia response pathways. A major advantage of this system is that these pathways can be manipulated in established xenograft tumors and thus more closely mimic the clinical situation as compared to conventional xenograft models. This system allows us to assess the potential of 
targeting these different pathways as well as targeting different points in the oxygen signaling pathways. An important aspect in this regard is that the xenograft tumors are established with the pathway of interest intact and then are induced with doxycycline to express interfering RNAs or transgenes to inactivate the oxygen signaling pathway. This eliminates any potential disturbances (i.e. on tumor take or growth) before tumors have completely formed. Most studies have been complicated by directly injecting gene knockout cells or their wild type controls. This might also explain some of the controversial results obtained for HIF-1a. Several groups have reported reduced tumor growth rates upon HIF-1a loss whereas others describe the opposite effect ${ }^{17-19}$. The tumor microenvironment furthermore has a major impact on the outcome of HIF$1 \alpha$ loss of function and potentially can affect other molecular targets in a similar manner ${ }^{20}$.

Here we successfully engineered U373 and HCT116 tumor cell lines to accept transgenes at a single genomic location. We were able to interfere with PERK/eIF2 $\alpha$, mTOR/eIF4F and HIF signaling by overexpression of either activating or inhibitory constructs (Figure 2). These three independent hypoxia responsive pathways each mediates different downstream processes and thus differentially influence hypoxic cell phenotype. Our data reveals that of these three pathways only HIF-1 $1 \alpha$ is important for maintaining proliferation rates in vitro under low oxygen tensions. This is in agreement with previously published data and might partly explain the reduced tumor growth rates of MEFs lacking HIF-1 $\alpha^{17,21}$. In contrast to the effect on proliferation all three pathways proved to be important for maintaining survival after a hypoxic insult. Of all tested constructs, overexpression of dominant negative eIF2 $\alpha$ (S51A) was most potent in sensitizing cells to hypoxia induced cell death. From published experiments with PERK knockout cells it was already known that phosphorylation of eIF $2 \alpha$ is important for cell survival during hypoxia ${ }^{9}{ }^{14}$. Our data support this and moreover suggest that there might be better ways to interfere with eIF $2 \alpha$ phosphorylation than targeting PERK. 


\section{Chapter 8}

The importance of mTOR signaling to eIF $4 F$ throught its ability to phosphorylate 4E-BP1 in determining hypoxia tolerance had not previously been investigated. However, clear evidence has been provided by several groups, including ours, that the MTOR pathway is inhibited upon exposure to hypoxia ${ }^{7}, 12,13$. Here we were able to show that interfering with the ability of hypoxia to suppress eIF4F via mTOR signaling indeed affects cell survival during hypoxia. However this only becomes evident after prolonged exposure periods ( $72 \mathrm{hr}$ ) in contrast to the relatively early effects we observe on cell survival when PERK/ eIF2 $\alpha$ or HIF signaling have been disrupted. This suggests that mTOR/ eIF4E might not be as important for cell survival during acute hypoxia as PERK/ eIF2 $\alpha$ and HIF signaling.

Interestingly, exposure to different levels of hypoxia did not alter the relative importance of a particular pathway in promoting hypoxia tolerance. This suggests that targeting PERK/eIF2 $\alpha$ or HIF signaling would be the most effective strategy at both extreme hypoxia $\left(<0.02 \% \mathrm{O}_{2}\right)$ and more moderate hypoxic $\left(0.2 \% \mathrm{O}_{2}\right)$ conditions. However, at this point we cannot rule out that one of the pathways might dominate cell survival at higher oxygen concentrations $\left(1.0-2.0 \% \mathrm{O}_{2}\right)$ or in the more relevant context of the tumor microenvironment. At these relatively higher oxygen tensions HIF- $1 \alpha$ is maximally activated and this therefore could shift the sensitivity more towards HIF targeting.

The complicated oxygenation status of solid tumors makes it virtually impossible to predict the best pathway from in vitro data. Tumor cells are exposed to acute, chronic and cycling levels of hypoxia as well as a range of different oxygen tensions ${ }^{15,16,22}$. Since this complex situation cannot be replicated in vitro it will be crucial to determine the most effective pathway from in vivo animal experiments. Furthermore, the relative contribution of the different hypoxia response pathways to phenotypes associated with hypoxia such as metastasis, angiogenesis, metabolism and genomic instability are unknown. Here we demonstrate that our model system is suitable to address these issues. We provided proof of 
principle that we can induce the expression of desired genes in established tumors using eGFP as a reporter. Animal experiments using xenograft tumors with defects in PERK/eIF2 $\alpha$, mTOR/ eIF4E and HIF signaling are ongoing to resolve the relative importance of these pathways for hypoxia tolerance. Finally, these results will determine which of these pathways will be the best candidate to target in combination with other cancer therapies.

\section{Acknowledgements}

We wish to acknowledge financial support from the Dutch Science Organization (ZonMW-NWO Top grant 912-03-047 to BW, and ZonMWVENI grant 016.056.015 to MK), the Dutch Cancer Society (KWF grant UM 2003-2821 to BW), and the EU $6^{\text {th }}$ framework program (Euroxy program to $\mathrm{BW})$.

\section{References}

1. Brown JM, Wilson WR: Exploiting tumour hypoxia in cancer treatment, Nat Rev Cancer 2004, 4:437-447

2. Semenza GL: Regulation of hypoxia-induced angiogenesis: a chaperone escorts VEGF to the dance, J Clin Invest 2001, 108:39-40

3. Hockel $M$, Schlenger $K$, Aral $B$, Mitze $M$, Schaffer U, Vaupel P: Association between tumor hypoxia and malignant progression in advanced cancer of the uterine cervix, Cancer Res 1996, 56:4509-4515

4. Erler JT, Cawthorne CJ, Williams KJ, Koritzinsky M, Wouters BG, Wilson C, Miller C, Demonacos C, Stratford IJ, Dive C: Hypoxia-mediated downregulation of Bid and Bax in tumors occurs via hypoxia-inducible factor 1dependent and -independent mechanisms and contributes to drug resistance, Mol Cell Biol 2004, 24:2875-2889

5. Hockel M, Vaupel P: Biological consequences of tumor hypoxia, Semin Oncol 2001, 28:36-41.

6. Wouters $B G$, van den Beucken $T$, Magagnin MG, Lambin $P$, Koumenis $C$ : Targeting hypoxia tolerance in cancer, Drug Resist Updat 2004, 7:25-40

7. Koritzinsky M, Magagnin MG, van den Beucken $T$, Seigneuric $R$, Savelkouls $K$, Dostie J, Pyronnet S, Kaufman RJ, Weppler SA, Voncken JW, Lambin P, Koumenis C, Sonenberg N, Wouters BG: Gene expression during acute and prolonged hypoxia is regulated by distinct mechanisms of translational control, Embo J 2006, 25:1114-1125

8. Kraggerud SM, Sandvik JA, Pettersen EO: Regulation of protein synthesis in human cells exposed to extreme hypoxia, Anticancer Res 1995, 15:683-686

9. Koumenis C, Naczki C, Koritzinsky M, Rastani S, Diehl A, Sonenberg N, Koromilas $A$, Wouters $B G$ : Regulation of protein synthesis by hypoxia via 


\section{Chapter 8}

activation of the endoplasmic reticulum kinase PERK and phosphorylation of the translation initiation factor eIF2alpha, Mol Cell Biol 2002, 22:7405-7416

10. van den Beucken $T$, Koritzinsky $M$, Wouters BG: Translational control of gene expression during hypoxia, Cancer Biol Ther 2006, 5:749-755

11. Koritzinsky $M$, Seigneuric $R$, Magagnin MG, van den Beucken $T$, Lambin $P$, Wouters BG: The hypoxic proteome is influenced by gene-specific changes in mRNA translation, Radiother Oncol 2005, 76:177-186

12. Connolly E, Braunstein S, Formenti S, Schneider RJ: Hypoxia inhibits protein synthesis through a 4E-BP1 and elongation factor 2 kinase pathway controlled by mTOR and uncoupled in breast cancer cells, Mol Cell Biol 2006, 26:39553965

13. Arsham AM, Howell J], Simon MC: A Novel Hypoxia-inducible Factorindependent Hypoxic Response Regulating Mammalian Target of Rapamycin and Its Targets, J. Biol. Chem. 2003, 278:29655-29660

14. Bi M, Naczki C, Koritzinsky $M$, Fels D, Blais J, Hu N, Harding $H$, Novoa I, Varia $M$, Raleigh J, Scheuner D, Kaufman RJ, Bell J, Ron D, Wouters BG, Koumenis $C$ : ER stress-regulated translation increases tolerance to extreme hypoxia and promotes tumor growth, Embo J 2005, 24:3470-3481

15. Cardenas-Navia LI, Yu D, Braun RD, Brizel DM, Secomb TW, Dewhirst MW: Tumor-dependent Kinetics of Partial Pressure of Oxygen Fluctuations during Air and Oxygen Breathing, Cancer Res 2004, 64:6010-6017

16. Bennewith $\mathrm{KL}$, Durand RE: Quantifying transient hypoxia in human tumor xenografts by flow cytometry, Cancer Res 2004, 64:6183-6189

17. Ryan HE, Poloni M, McNulty W, Elson D, Gassmann M, Arbeit JM, Johnson RS: Hypoxia-inducible factor-1alpha is a positive factor in solid tumor growth, Cancer Res 2000, 60:4010-4015

18. Maxwell PH, Dachs GU, Gleadle JM, Nicholls LG, Harris AL, Stratford IJ, Hankinson O, Pugh CW, Ratcliffe PJ: Hypoxia-inducible factor-1 modulates gene expression in solid tumors and influences both angiogenesis and tumor growth, Proc Natl Acad Sci U S A 1997, 94:8104-8109

19. Carmeliet $P$, Dor $Y$, Herbert JM, Fukumura D, Brusselmans $K$, Dewerchin $M$, Neeman $M$, Bono $F$, Abramovitch $R$, Maxwell $P$, Koch $C J$, Ratcliffe $P$, Moons $L$, Jain RK, Collen D, Keshert E, Keshet E: Role of HIF-1alpha in hypoxiamediated apoptosis, cell proliferation and tumour angiogenesis, Nature 1998, 394:485-490

20. Blouw B, Song H, Tihan T, Bosze J, Ferrara N, Gerber HP, Johnson RS, Bergers $G$ : The hypoxic response of tumors is dependent on their microenvironment, Cancer Cell 2003, 4:133-146

21. Seagroves TN, Ryan HE, Lu H, Wouters BG, Knapp M, Thibault $P$, Laderoute $K$, Johnson RS: Transcription factor HIF-1 is a necessary mediator of the pasteur effect in mammalian cells, Mol Cell Biol 2001, 21:3436-3444

22. Bussink J, Kaanders $\mathrm{JH}$, van der Kogel AJ: Tumor hypoxia at the microregional level: clinical relevance and predictive value of exogenous and endogenous hypoxic cell markers, Radiother Oncol 2003, 67:3-15 


\section{CHAPTER 9}

\section{General discussion and summary}

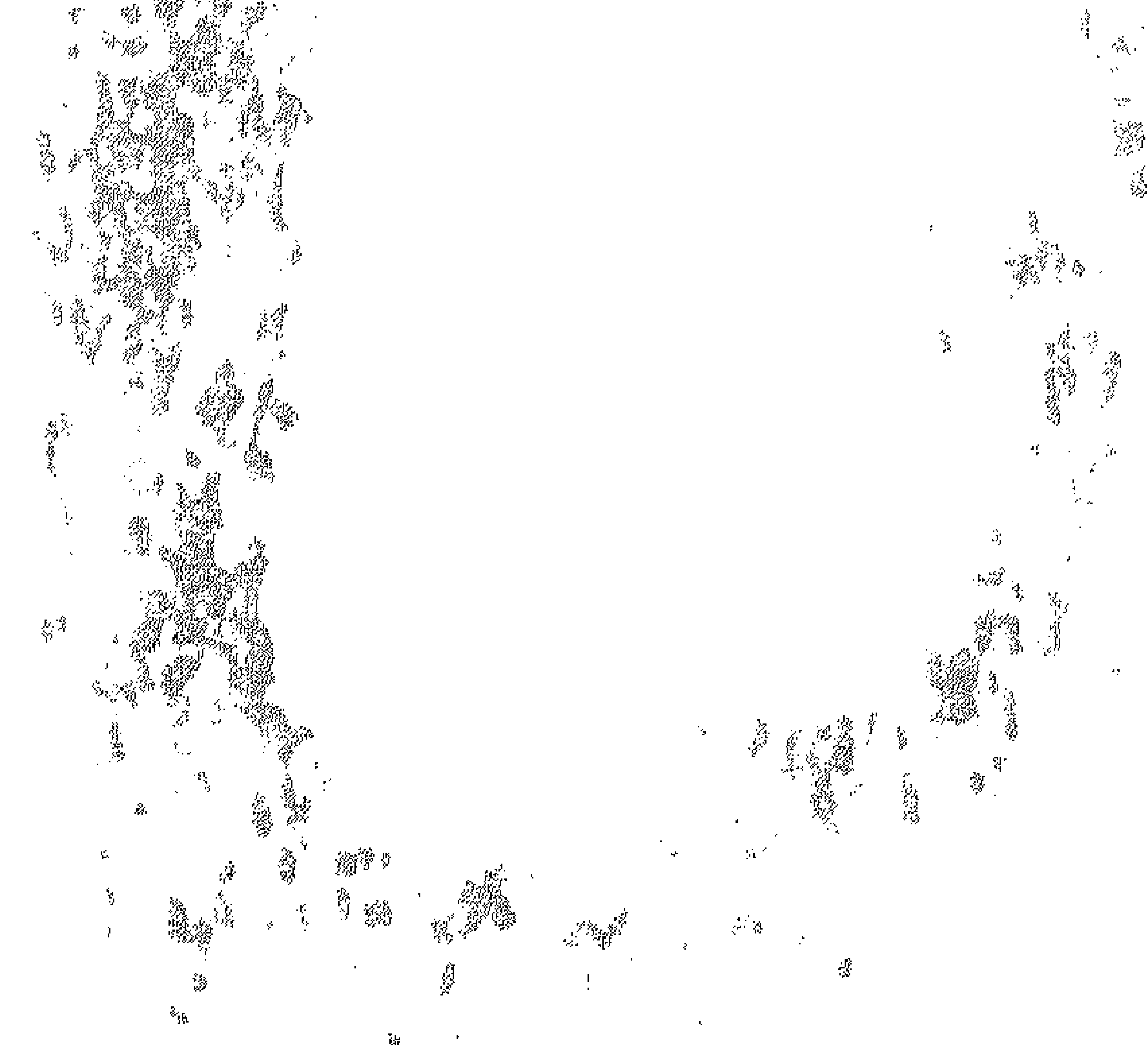




\section{General discussion and summary}

This thesis describes the consequences of regulating mRNA translation on gene expression during hypoxia. It has been known for more than a decade that hypoxia causes a profound drop in the rate of overall protein synthesis $^{1,2}$. In 2002 and just prior to the start of this thesis it was found that this reduction in protein synthesis was due to a HIF independent, oxygen-sensitive pathway that results in direct inhibition of mRNA translation $^{3}$. This initial publication also demonstrated that phosphorylation of eIF $2 \alpha$ plays an important role in the repression of overall mRNA translation. As described in the general introduction, eukaryotic translation is a highly regulated process which involves many eIFs and complicated signaling cascades. This fact and preliminary data from our lab led us to hypothesize that translational control is mediated through several distinct pathways during hypoxia.

In the study described in chapter 2 we were able to define the contribution of eIF $2 \alpha$ phosphorylation to translational control in hypoxic cells. Using MEF cells that are compromised in their ability to phosphorylate eIF $2 \alpha$ upon hypoxia, we showed that this pathway was responsible for the majority of the initial drop in protein synthesis, but that additional pathways contributed to translational repression especially during chronic exposure. As hypothesized, we found an additional hypoxia sensitive regulatory step in the cap-binding complex eIF4F. Our data shows that the formation of eIF4F is severely compromised during hypoxia by different mechanisms. First, prolonged exposure to hypoxic conditions leads to dephosphorylation of 4E-BP1. This hypophosphorylation results in an increased affinity for the cap-recognizing protein eIF4E. As a consequence, eIF4E is unable to participate in the formation of eIF4F and translation initiation is repressed.

Regulation of 4E-BP1 is controlled by the kinase mTOR which integrates signals from several upstream pathways. Arsham and colleagues have previously shown that 4E-BP1 hypophosphorylation is the result of mTOR inhibition during moderate hypoxia in combination with 
serum deprivation ${ }^{4}$. In a recent paper Liu and colleagues demonstrate that moderate hypoxia causes repression of mTOR through activation of $A M P K^{5}$. Strikingly, this only affects overall protein synthesis rates after hypoxic exposure periods of over 24 hours. From these studies it remains unclear what the relative contribution of MTOR inhibition to the overall inhibition of mRNA translation observed during hypoxia really is. This needs to be addressed in the following years. Likewise it will be interesting to establish the role of MTOR in hypoxia tolerance and hypoxia-induced gene expression.

The observation that disruption of eIF4F took place prior to complete dephosphorylation of 4E-BP1 led us to the discovery of a new interesting step in the regulation of eIF4F. We were able to show that eIF4F formation is hampered by translocation of eIF4E together with the shuttling factor $4 \mathrm{E}-\mathrm{T}$ into the nucleus or to recently discovered cytoplasmic bodies of mRNA processing (P-bodies) ${ }^{6,7}$. Although the exact mechanism of how this takes place is unclear, our data suggests that dephosphorylation of $4 \mathrm{E}-\mathrm{T}$ might be important. Thus, as hypothesized hypoxia leads to the activation of at least two distinct pathways that inhibit mRNA translation. A rapid, transient response through phosphorylation of eIF $2 \alpha$ and a prolonged effect through disruption of eIF4F.

In chapter 2 we also showed for a variety of genes that gene-specific translation is modulated by hypoxia. This data led us to our second hypothesis that translational control plays an important role in mediating rapid changes in gene expression upon hypoxia (chapter 4 ). To test this hypothesis we assessed the hypoxic phenotype after acute $(1 \mathrm{~h})$ and chronic (24 h) hypoxia using a proteomic approach. Remarkable changes in the expression of specific proteins were observed after only 1 hour of hypoxic exposure. Due to the short exposure time it is very unlikely that these changes are due to a transcriptional response. These data suggest that mechanisms other than transcription are responsible for the observed proteomic changes. 


\section{Chapter 9}

To investigate whether rapid changes in protein expression might be mediated through modulation of gene-specific mRNA translation we applied a technique that allowed us to assess translation efficiency on a genome-wide scale. For this purpose, both total mRNA as well as efficiently translated mRNAs from hypoxia exposed cells was hybridized to Affymetrix microarrays. Using a similar technique, Blais et al. had identified multiple preferentially translated genes during prolonged hypoxia $(16 \mathrm{~h})^{8}$. As described above, at this time point translation inhibition is predominantly regulated through disruption of eIF4F. Since we were interested to determine genes affected by both eIF $2 \alpha$ and eIF4F regulation we performed a similar study after acute hypoxia $(4 \mathrm{~h}$ ) (chapter 4). We were able to show that of the top 50 genes with the largest induction during hypoxia, $20 \%$ displayed a selective ability to be translated $^{9}$. This included several genes dependent on HIF-1 or involved in the UPR. These data showed that regulation of gene-specific mRNA translation contributes to the hypoxic proteome.

One important question that was raised by these data was what the relative contributions of transcriptional and translational control are on overall changes in gene expression during hypoxia. To address this issue we studied the effect of hypoxia on gene expression in more detail. Since the activation of these mechanisms during hypoxia are complex and operate with different kinetics (chapter 2), we extended our pilot study described above by assessing mRNA translation as a function of hypoxic exposure time (chapter 5 ). Our results reveal that translational regulation contributes predominantly to gene induction during acute hypoxia, whereas transcriptional control seems more important after prolonged hypoxic exposure. In contrast, translational control appears to be the main mechanism for down regulation of gene expression during hypoxia. These data suggest that translational control may be especially important during acute hypoxia. Translationally regulated genes reach a minimum after 8 hours of hypoxia, and then increase again with a kinetic pattern that is consistent with the disruption of eIF4F. Altogether our data 
demonstrates that gene expression regulated through translational control during hypoxia is a highly dynamic process. This might be important for the adaptive response during acute hypoxia. Especially since oxygenation levels within the tumor can fluctuate dramatically over very short periods of time that are often too short to elicit effective transcriptional response ${ }^{10}$. Hypoxia down regulated mRNA translation rates of genes encoding for translation factors, ribosomal proteins as well as proteins involved in cell growth. We believe that this might lead to a more persistent inhibition of mRNA translation compared to the transient repression by eIF $2 \alpha$ phosphorylation and eIF4F disruption. Whether this might provide tumor cells with a survival mechanism to tolerate long term hypoxic stress needs to be further evaluated. On the other hand hypoxia stimulated mRNA translation of genes involved in signal transduction, metabolism and transcriptional regulation. The possibility that translational control regulates the transcriptional response during hypoxia is an interesting finding and will be worthwhile to explore in the future.

One of the genes that we characterized in more detail, Cited2, was identified as one of the top translationally regulated genes in our gene profiling studies (chapter 4 and 5). Cited 2 is interesting because it had been proposed to act as negative regulator of HIF-1 transcriptional activity by antagonizing its interaction with coactivator $\mathrm{CBP} / \mathrm{p} 300^{15,16}$. In chapter 6 we provided the first clear evidence using HIF-1 $\alpha$ null cells that Cited 2 is indeed a genuine HIF-1 $\alpha$ target and thus can act as postulated antagonist. We were able to validate the gene expression results and showed that the translational and transcriptional inductions mimic each other closely. This results in a net increase in Cited 2 protein synthesis as the overall protein synthesis in the cell drops. To find an explanation for the preferential translation of Cited 2 we analyzed the $5^{\prime} U T R$ sequences from different species and discovered an evolutionary conserved UORF. As discussed earlier, this RNA element is essential for gene specific translation of ATF4 during hypoxia. We were able to show that Cited 2 translation is not impaired in cells that have lost the ability to induce translation of UORF 


\section{Chapter 9}

regulated genes like ATF4. Despite of this, our data shows that translational control regulates the expression of Cited2 during hypoxia and thus indirectly affects HIF-1 transcriptional activity. This study also indicates that cross-talk between transcriptional and translational changes during hypoxia may be more ubiquitous than previously recognized.

In chapter 7 we followed up on one of the leads we obtained from an ongoing screen in our laboratory, CA9. This screen involves characterization of UPR mediated changes in gene expression during hypoxia by transcriptional profiling of wild-type and eIF2 $\alpha$ S51A mutant MEFs. We identified CA9 as one of the top differentially regulated genes between the WT and S51A cells. Using different model systems we were able to show that expression of CA9 was dependent on the cells capacity to phosphorylate eIF2 $\alpha$. We were able to show that PERK/eIF2 $\alpha$ translationally regulated gene ATF4 binds directly to the CA9 promoter and that this was associated with loss of the transcriptional repressive histone 3 lysine 27 (H3K27) tri-methylation mark. We furthermore confirmed the role of ATF4 in regulating CA9 during hypoxia by transient knock-down or over expression of ATF4. This study indicates that CA9 expression is mediated through independent activation of both the HIF-1 and UPR hypoxia response pathways. These data may have important implications for the use of CA9 as a diagnostic tumor marker.

In chapter 8 we described the development of a novel tumor model designed to investigate the relative importance of different hypoxia response pathways. The rational behind this new tumor model is that we can manipulate these hypoxia responsive pathways in established xenograft tumors. This model allows us to more accurately determine the importance of the various pathways compared to conventional xenograft models. Our in vitro data showed that PERK/eIF2 $\alpha$, mTOR/eIF4F and HIF signaling independently contribute to hypoxia tolerance as their disruption significantly sensitized cells to hypoxia induced cell death. Finally, we demonstrated that this isogenic inducible system can be used to efficiently express transgenes in established xenograft tumors by administration of

\section{4}


doxycline to the drinking water of tumor bearing mice. This enables us in the future to determine the relative importance of targeting these pathways in vivo in pre-established tumors in a manner that closely mimics the therapeutic situation.

\section{Concluding remarks and future perspectives}

Translational control has emerged as an important adaptive response to hypoxia by changing the hypoxic phenotype and increasing the cellular tolerance towards this condition. Modulation of mRNA translation affects essential hypoxia regulated transcription factors like HIF and ATF4. Consequently, this also causes changes in the expression of many downstream genes in an indirect manner. The discovery of the pathways that mediate translational control in response to hypoxia has provided insight into the link between translational control and hypoxia tolerance. However, as often new findings raise more research questions. One important question to resolve in the coming years is how specific mRNA species become preferentially translated during hypoxia. There is good evidence that suggests a pivotal role for the untranslated regions of mRNA transcripts in their ability to become translated. It will be interesting to identify RNA elements that stimulate mRNA translation on a genome-wide scale. For this it will be very useful to use genetic models in which particular translational control pathways have been disrupted. For example, the MEF S51A model is an excellent model to identify genes regulated by uORFs like ATF4, as its induction is abolished under hypoxia in S51A MEF cells compared to the wild type controls. However these kind of studies need further validation on an individual basis. This will be a difficult task, requiring a lot of effort. Even for a widely studied gene, like HIF-1 $\alpha$ which is it has been proven difficult to unravel its translational regulation and it still remains partly unclear. In addition to IRES activity and UORFs it is has recently become clear that changes in translation of specific genes may also result from expression of a class of small noncoding RNAs called microRNAs (miRNAs) ${ }^{17}$. Interestingly, two recent 


\section{Chapter 9}

studies have shown that hypoxia alters the expression pattern of known miRNAs, although the importance of these miRNAs and their targets on the hypoxic phenotype remain unknown ${ }^{18,19}$. An interesting hypothesis to investigate in the future is that regulation of miRNA expression during hypoxia contributes to changes in mRNA translation and that this is relevant for cell phenotype and tumor growth.

A second point of research will be to address to which degree translational control pathways are altered in cancer. This is important if we consider these pathways as new therapeutic targets. Especially for the eIF4F dependent response, since the upstream signaling pathways are often mutated in tumors. Furthermore, it will be interesting to sort out the relative importance of the various known hypoxia responsive pathways on cancer growth and treatment response in the context of a real tumor microenvironment. This will provide guidance in developing new types of therapies aimed at targeting tumor hypoxia and its cellular response. We made some progress on this by developing an inducible model system in which the different hypoxia response pathways can be inactivated. Efforts are currently underway to determine the relative importance of these pathways in xenograft tumor models. Although these issues remain to be addressed it certainly does not slow down the development of these novel therapeutic agents by the pharmaceutical industry. Finally the success of these new therapeutic drugs will be dependent upon their careful and logical introduction into the clinic. Since hypoxia is highly heterogeneous within the tumour it is crucial to consider targeting hypoxia only in combination with other traditional treatments.

\section{References}

1. Pettersen EO, Juul NO, Ronning OW: Regulation of protein metabolism of human cells during and after acute hypoxia, Cancer Res 1986, 46:4346-4351

2. Kraggerud SM, Sandvik JA, Pettersen EO: Regulation of protein synthesis in human cells exposed to extreme hypoxia, Anticancer Res 1995, 15:683-686

3. Koumenis C, Naczki C, Koritzinsky M, Rastani S, Diehl A, Sonenberg N, Koromilas A, Wouters BG: Regulation of protein synthesis by hypoxia via 
activation of the endoplasmic reticulum kinase PERK and phosphorylation of the translation initiation factor eIF2alpha, Mol Cell Biol 2002, 22:7405-7416

4. Arsham AM, Howell J], Simon MC: A Novel Hypoxia-inducible Factorindependent Hypoxic Response Regulating Mammalian Target of Rapamycin and Its Targets, J. Biol. Chem. 2003, 278:29655-29660

5. Liu L, Cash TP, Jones RG, Keith B, Thompson CB, Simon MC: Hypoxia-induced energy stress regulates mRNA translation and cell growth, Mol Cell 2006, 21:521-531

6. Dostie J, Ferraiuolo M, Pause A, Adam SA, Sonenberg N: A novel shuttling protein, $4 E-T$, mediates the nuclear import of the $M R N A 5^{\prime}$ cap-binding protein, eIF4E, Embo J 2000, 19:3142-3156

7. Andrei $M A$, Ingelfinger $D$, Heintzmann $R$, Achsel $T$, Rivera-Pomar $R$, Luhrmann $\mathrm{R}$ : A role for eIF4E and eIF4E-transporter in targeting mRNPs to mammalian processing bodies, Rna 2005, 11:717-727

8. Blais JD, Filipenko $\mathrm{V}$, Bi M, Harding HP, Ron D, Koumenis C, Wouters BG, Bell JC: Activating transcription factor 4 is translationally regulated by hypoxic stress, Mol Cell Biol 2004, 24:7469-7482

9. Koritzinsky $M$, Seigneuric $R$, Magagnin $M G$, van den Beucken $T$, Lambin $P$, Wouters BG: The hypoxic proteome is influenced by gene-specific changes in mRNA translation, Radiother Oncol 2005, 76:177-186

10. Cardenas-Navia LI, Yu D, Braun RD, Brizel DM, Secomb TW, Dewhirst MW: Tumor-dependent Kinetics of Partial Pressure of Oxygen Fluctuations during Air and Oxygen Breathing, Cancer Res 2004, 64:6010-6017

11. Lang KJ, Kappel A, Goodall G]: Hypoxia-inducible factor-1alpha mRNA contains an internal ribosome entry site that allows efficient translation during normoxia and hypoxia, Mol Biol Cell 2002, 13:1792-1801

12. Stein I, Itin A, Einat $P$, Skaliter R, Grossman $Z$, Keshet E: Translation of vascular endothelial growth factor mRNA by internal ribosome entry: implications for translation under hypoxia, Mol Cell Biol 1998, 18:3112-3119

13. Thoma C, Bergamini G, Galy B, Hundsdoerfer P, Hentze MW: Enhancement of IRES-mediated translation of the c-myc and BiP mRNAs by the poly $(A)$ tail is independent of intact eIF4G and PABP, Mol Cell 2004, 15:925-935

14. Suzuki $Y$, Ishihara $D$, Sasaki $M$, Nakagawa $H$, Hata $H$, Tsunoda $T$, Watanabe $M$, Komatsu $T$, Ota $T$, Isogai $T$, Suyama A, Sugano S: Statistical analysis of the $5^{\prime}$ untranslated region of human mRNA using "Oligo-Capped" CDNA libraries, Genomics 2000, 64:286-297

15. Bhattacharya $S$, Michels $C L$, Leung $M K$, Arany ZP, Kung $A L$, Livingston $D M$ : Functional role of p35srj, a novel p300/CBP binding protein, during transactivation by HIF-1, Genes Dev 1999, 13:64-75

16. Yin Z, Haynie J, Yang $X$, Han B, Kiatchoosakun S, Restivo J, Yuan S, Prabhakar NR, Herrup K, Conlon RA, Hoit BD, Watanabe M, Yang YC: The essential role of Cited2, a negative regulator for HIF-1alpha, in heart development and neurulation, Proc Natl Acad Sci U S A 2002, 99:10488-10493

17. Bartel DP: MicroRNAs: genomics, biogenesis, mechanism, and function, Cell 2004, 116:281-297

18. Kulshreshtha R, Ferracin $M$, Wojcik SE, Garzon R, Alder $H$, Agosto-Perez FJ, Davuluri $R$, Liu CG, Croce CM, Negrini $M$, Calin GA, Ivan M: A microRNA signature of hypoxia, Mol Cell Biol 2007, 27:1859-1867

19. Hebert C, Norris K, Scheper MA, Nikitakis N, Sauk J]: High mobility group A2 is a target for miRNA-98 in head and neck squamous cell carcinoma, Mol Cancer $2007,6: 5$ 


\section{Algemene discussie en samenvatting}

In dit proefschrift zijn de gevolgen van translationele regulatie op gen expressie tijdens hypoxie beschreven. Het is al meer dan tien jaar bekend dat hypoxie een grote daling in totale eiwit synthese veroorzaakt ${ }^{1,2}$. In 2002, vlak voor de start van dit promotieonderzoek, werd bekend dat deze reductie in eiwit synthese door een HIF onafhankelijk, zuurstofgevoelig mechanisme wordt gereguleerd dat resulteert in een directe inhibitie van mRNA translatie ${ }^{3}$. Deze initiële publicatie demonstreerde ook dat fosforylatie van eIF2 $\alpha$ een belangrijke rol speelt in de remming van totale mRNA translatie. Zoals beschreven in de algemene introductie, is eukaryotische translatie een strict gereguleerd proces waarin vele eIFs en ingewikkelde signaal transductie routes een rol spelen. Dit feit en preliminaire data van ons lab hebben geleid tot de hypothese dat translationele regulatie wordt veroorzaakt door verscheidene mechanismen tijdens hypoxie.

In hoofdstuk 2 wordt een studie beschreven waarin de bijdrage van eIF $2 \alpha$ fosforylatie op translationele controle in hypoxische cellen wordt gedefinieerd. Door MEF cellen te gebruiken die niet in staat zijn om eIF2 $\alpha$ te fosforyleren tijdens hypoxie, konden we aantonen dat dit mechanisme grotendeels verantwoordelijk is voor de initiële daling in eiwit synthese. $\mathrm{Er}$ blijken echter additionele mechanismen actief die ook zorgen voor remming van mRNA translatie voornamelijk tijdens chronische blootstelling aan hypoxie. Zoals veronderstelt, hebben we een extra regulatie mechanisme gevonden in het cap-binding complex eIF4F. Onze data laat zien dat de formatie van eIF4F ernstig belemmerd wordt door verschillende mechanismen tijdens hypoxie. Ten eerste, een langdurige blootstelling aan hypoxische condities leidt tot defosforylatie van 4E-BP1. Deze hypofosforylatie resulteert in een verhoogde affiniteit voor het capherkennende eiwit eIF4E. Het resultaat hiervan is dat eIF4E niet meer deel kan nemen in de formatie van eIF4F en de initiatie van translatie wordt geremd. 


\section{Algemene discussie en samenvatting}

De regulatie van 4E-BP1 wordt gecontroleerd door de kinase mTOR die signalen integreert van verschillende bovenliggende mechanismen. Arsham en collega's hebben al eerder laten zien dat 4E-BP1 hypofosforylatie het resultaat is van mTOR inhibitie tijdens matige hypoxie in combinatie met serum depletie ${ }^{4}$. In een recent onderzoeksartikel beschrijven Liu en collega's dat matige hypoxie onderdrukking van mTOR veroorzaakt door AMPK te activeren ${ }^{5}$. Opmerkelijk is dat dit alleen effect heeft op totale eiwit synthese na blootstelling aan hypoxische condities voor perioden langer dan 24 uur. Na deze studies blijft het onduidelijk wat de relatieve bijdrage van mTOR inhibitie op de totale remming van mRNA translatie waargenomen tijdens hypoxie werkelijk is. Verder onderzoek is nodig om dit te verduidelijken. Evenzo zal het interessant zijn om te onderzoeken hoe mTOR de gevoeligheid voor hypoxie en hypoxiegeïnduceerde gen expressie beïnvloed.

De observatie dat eIF4F desintegreerd voordat 4E-BP1 volledig gedefosforyleerd was leidde tot de ontdekking van een nieuwe interessante controle punt in de regulatie van eIF4F. We hebben kunnen aantonen dat eIF4F formatie wordt voorkomen door translocatie van eIF4E samen met de shutteling factor 4-ET naar de nucleus of naar de recent ontdekte cytoplasmatische bodies waar mRNA processing (P-bodies) plaatsvindt ${ }^{6,7}$. Hoewel het exacte mechanisme nog onduidelijk is, wijst onze data erop dat defosforylatie van 4-ET belangrijk kan zijn. Derhalve, zoals veronderstelt leidt hypoxie tot de activatie van tenminste twee duidelijke mechanismen die mRNA translatie remmen. Een snelle, tijdelijke reactie door fosforylatie van eIF2 $\alpha$ en een langdurig effect door eIF4F desintegratie.

In hoofdstuk 2 hebben we voor verscheidene genen laten zien dat genspecifieke translatie wordt gemoduleerd door hypoxie. Deze data heeft geleid tot onze tweede hypothese, dat translationele controle een belangrijke rol speelt in het veroorzaken van snelle veranderingen in gen expressie tijdens hypoxie (hoofdstuk 4). Om deze hypothese te testen hebben we bepaald wat het hypoxische fenotype is na acute (1 uur) en 
Algemene discussie en samenvatting

chronische (24 uur) hypoxie. Opmerkelijke veranderingen in eiwit expressie werden al 1 uur na blootstelling aan hypoxie waargenomen. Vanwege de korte blootstellingtijd is het erg onwaarschijnlijk dat deze veranderingen tot stand komen door een transcriptionele inductie. Deze data duiden erop dat er andere mechanismen dan transcriptie verantwoordelijk zijn voor de waargenomen veranderingen in eiwit expressie.

Om te onderzoeken of snelle veranderingen in eiwit expressie veroorzaakt worden door veranderingen in gen-specifieke mRNA translatie, hebben we een techniek toegepast die ons in staat stelt om translatie efficiëntie op een genoomwijde schaal te bepalen. Voor dit doeleinde, werd zowel totaal mRNA als efficiënt getransleerd mRNA van cellen die blootgesteld waren aan hypoxie, gehybridizeerd op Affymetrix microarrays. Gebruik makend van dezelfde techniek, hebben Blais et al. meerdere specifiek getransleerde genen tijdens hypoxie (16 uur) geïdentificeerd $^{8}$. Zoals hierboven beschreven, is op dit tijdpunt translatie inhibitie voornamelijk gereguleerd door desintegratie van eIF4F. Omdat we graag genen wilden identificeren die door zowel eIF2 $\alpha$ als eIF4F gereguleerd worden hebben we een vergelijkbare studie uitgevoerd na acute hypoxie (4 uur) (hoofstuk 4). Hierin hebben we kunnen aantonen dat van de top 50 genen met de hoogste inductie tijdens hypoxie, 20\% een selectief vermogen om getransleerd te kunnen worden lieten zien ${ }^{9}$. Hierbij inbegrepen waren meerdere genen die afhankelijk zijn van HIF-1 of die in rol spelen in de UPR. Deze data laten zien dat regulatie van genspecifieke mRNA translatie bijdraagt aan het hypoxische fenotype.

De relatieve bijdrage van transcriptionele en translationele controle op de totale veranderingen in gen expressie tijdens hypoxie bleef onbekend. Om hier meer inzicht in te krijgen hebben we in meer detail gekeken naar de effecten van hypoxie op gen expressie. Aangezien de activatie van de mechanismen die mRNA translatie beinvloeden tijdens hypoxie zeer complex is (hoofdstuk 2), hebben we ervoor gekozen om onze pilot studie, zoals hierboven beschreven uit te breiden door mRNA translatie te 


\section{Agemene discussie en samenvathing}

bepalen als functie van hypoxische blootstellingtijd (hoofdstuk 5). Onze resultaten tonen aan dat translationele regulatie voornamelijk een rol speelt bij gen expressie tijdens acute hypoxie, terwijl transcriptionele controle belangrijker is na langdurige blootstelling aan hypoxie. Verder lijkt translationele controle het belangrijkste mechanisme te zijn voor de onderdrukking van gen expressie tijdens hypoxie. Dit wijst erop dat translationele controle vooral belangrijk is tijdens acute hypoxie. Het aantal translationeel gereguleerde genen bereiken een minimum na 8 uur hypoxie en neemt dan weer toe met een kinetisch patroon dat gelijk is aan de desintegratie van eIF4F. Gezamenlijk toont onze data aan dat gen expressie gereguleerd door translationele controle tijdens hypoxie een zeer dynamisch proces is. Hierdoor kan het functioneren als een belangrijk aanpassings mechanisme voor tumor cellen, aangezien zuurstof concentraties in tumoren dramatisch kunnen veranderen over een heel korte tijd ${ }^{10}$. Onze data laat zien tijdens hypoxia mRNA translatie specifiek geremt wordt voor transcripten die coderen voor transcriptie factoren, ribosomale eiwitten en eiwitten betrokken bij cel groei. Dit zou mogelijk kunnen leiden tot een meer langdurigere inhibitie van mRNA translatie vergeleken met de tijdelijke remming veroorzaakt door verstoring van eIF4F en eIF $2 \alpha$ fosforylatie. Of dit tumor cellen in staat stelt om langdurige blootstelling aan hypoxische condities te overleven moet verder onderzocht worden. Aan de ander kant tonen onze resultaten aan dat hypoxia de mRNA translatie van genen stimuleerd die betrokken zijn bij signaal transductie, metabolisme en de regulatie van transcriptie. Dit suggereerd dat de transcriptionele respons tijdens hypoxia beinvloed wordt door regulatie van mRNA translatie. Deze hypothese is zeer interessant voor verder onderzoek.

Een van de genen die we in meer detail bestudeerd hebben, Cited2, hebben we geïdentificeerd in onze microarray studies als een van de meest translationeel gereguleerde genen (hoofdstuk 4 en 5). Dit is zeer interessant aangezien Cited 2 mogelijk de transcriptionele activiteit van HIF-1 negatief beïnvloed door de interactie met coactivator CBP/p300 te 


\section{Algemene discussie en samenvarting}

verhinderen ${ }^{15,16}$. In hoofdstuk 6 tonen we eenduidig aan dat Cited 2 mRNA expressie afhankelijk is van HIF-1 $1 \alpha$ en dat Cited 2 dus als negatief respons mechanisme voor HIF activatie kan dienen. We hebben de microarray data succesvol gevalideerd en aangetoond dat Cited 2 zowel transcriptioneel als translationeel in dezelfde mate geïnduceerd wordt. Dit resulteerd in een netto toename van Cited2 eiwit synthese aangezien de totale eiwit synthese in de cel afneemt. Om deze preferentiele mRNA translatie te verklaren hebben wij de niet-coderende gedeeltes van het Cited2 transcript verder onderzocht. In het $5^{\prime}$ gedeelte troffen we een evolutioneer geconserveerd UORF aan. Zoals eerder bespoken stimuleert dit RNA element ondermeer translatie van de hypoxia gereguleerde transcriptie factor ATF4. Onze resultaten tonen echter aan dat het UORF in Cited2 niet de translatie bevorderd zoals bij ATF4. Desondanks laat deze data zien dat de regulatie van mRNA translatie HIF-1 activiteit indirect beïnvloed via Cited2. Tevens blijkt hieruit dat transcriptionele en translationele veranderingen elkaar meer beïnvloeden dan voorheen gedacht.

In hoofdstuk 7 hebben wij het eiwit CA9 verder bestudeerd dat tijdens een nog voortdurende screening in ons laboratorium opgepikt is. Deze screening is gebaseerd op het bepalen van transcriptie profielen in WT en S51A MEF cellen. CA9 was een van de genen die een zeer groot expressie verschil vertoonde tussen de WT en S51A MEFs. In verschillende model systemen hebben wij laten zien dat CA9 expressie afhankelijk is van eIF2 $\alpha$ fosforylatie. We hebben kunnen aantonen dat de PERK/eIF2 $\alpha$ afhankelijke transcriptie factor ATF4 bind aan de promoter regio van CA9. De CA9 promoter toonde tevens een verlies van histon 3 lysine 27 (H3K27) trimethylatie aan, een histon modificatie die geassocieerd is met inhibitie van transcriptie. De betrokkenheid van ATF4 in de regulatie van CA9 hebben we verder gevalideerd door tijdelijke uitschakeling of overexpressie van ATF4. Onze studie toont aan dat CA9 expressie onafhankelijk gereguleerd wordt door HIF-1 $\alpha$ en de UPR. Deze resultaten 
zijn mogelijk van groot belang wanneer CA9 als een diagnostische tumor marker gebruikt zou worden.

In hoofdstuk 8 beschrijven we de ontwikkeling van een nieuw tumor model waarmee het belang van verschillende hypoxia respons mechanismen bepaald kan worden. In dit nieuwe tumor model kunnen deze mechanismen uitgeschakelt worden in reeds ontwikkelde xenograft tumoren. Dit stelt ons in staat om het belang van de verschillende hypoxia respons mechanismen beter te bepalen vergeleken met de conventionele xenograft modellen. Onze in vitro data toont aan dat PERK/eIF2 $\alpha$, mTOR/eIF4F en HIF allen bijdragen aan de gevoeligheid voor hypoxische condities. Tot slot hebben we laten zien dat dit induceerbaar isogeen model gebruikt kan worden voor het tot expressie brengen van transgenen in reeds ontwikkelde xenograft tumoren. Hiermee kunnen we in de toekomst op een meer relevante manier bepalen welke van de verschillende hypoxia respons mechanismen geschikt is als aangrijpingspunt voor een mogelijk nieuwe therapie.

\section{Conclusies en toekomst perspectieven}

Regulatie van mRNA translatie manifesteert zich als een belangrijk mechanisme dat door tumor cellen gebruikt wordt om de gevoeligheid voor hypoxische condities te verminderen. Veranderingen in mRNA translatie hebben rechstreeks invloed op belangrijke transcriptie factoren zoals HIF en ATF4. Indirect beinvloed dit de expressie van een groot aantal genen die door deze transcriptie factoren gereguleerd worden. Het ontdekken van de mechanismen die mRNA translatie reguleren tijdens hypoxie heeft geleid tot nieuwe inzichten in de relatie tussen mRNA translatie en de gevoeligheid voor hypoxische condities. Natuurlijk leidt deze ontdekking tot nieuwe vraagstellingen. Zo zal het interessant zijn om te onderzoeken hoe specifieke mRNA transcripten actief getransleerd blijven terwijl translatie van het merendeel van mRNAs geremt is. Er zijn duidelijke aanwijzigingen dat de niet-coderende gedeeltes van een mRNA 
transcript hierin een rol spelen. Of er specifieke RNA elementen bestaan in de niet-coderende mRNA gedeeltes die mRNA translatie stimuleerd moet verder bepaald worden. Hiervoor zouden microarrays uitgevoerd kunnen worden met cel modellen waarin specifieke mRNA regulatie mechanismen uitgeschakeld zijn. Zo zou het MEF S51A model ideaal zijn om mRNA transcripten te identificeren die voor actieve translatie afhankelijk zijn van UORFs in hun niet-coderende mRNA. Transiatie van deze mRNAs wordt namelijk niet geinduceerd in de S51A MEFs vergeleken met de WT MEFs. Aangezien alle geïdentificeerde genen op individuele basis gevalideerd moeten worden zal dit veel tijd en inzet vergen. Zelfs voor veelvuldig

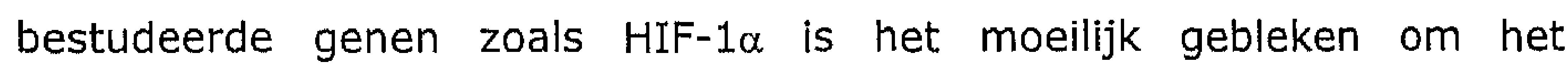
mechanisme dat translatie van HIF-1 $\alpha$ mRNA mogelijk maakt te bepalen en blijft het gedeeltelijk onbekend. Naast de reeds bekende IRES en UORF elementen is recent gebleken dat mRNA translatie ook beinvloed kan worden door expressie van kleine, niet-coderende mRNA moleculen die microRNAs (miRNAs) worden genoemd ${ }^{17}$. Uit twee recente studies is gebleken dat de expressie van bekende miRNAs beinvloed word door hypoxia ${ }^{18,}{ }^{19}$. Hoewel deze resultaten zeer interessant zijn blijft het momenteel onbekend of dit ook het hypoxische fenotype van de cel veranderd. De gedachte dat veranderingen in miRNA expressie translatie van specifieke mRNA transcripten beinvloed die van belang zijn voor het hypoxische fenotype en tumor ontwikkeling is een interessante hypothese om verder te bestuderen.

Een ander interessant onderwerp voor verder onderzoek is om te bepalen in hoe verre de mechanismen die mRNA translatie beinvloeden veranderd zijn in tumoren. Informatie hierover is van groot belang als we nieuwe behandelswijzen willen baseren op deze mechanismen. In het bijzonder voor de elF4F afhankelijke respons aangezien de bovenliggende signaal transductie routes vaak veranderd zijn in tumoren. Verder zal het belangrijk zijn om te achterhalen welk hypoxia respons mechanisme het meest interessant is om een nieuwe therapie op te richten. Belangrijk hierbij is dat dit bepaald wordt in de context van een echt tumor 
micromilieu. Dit bevorderd de ontwikkeling van een nieuw soort therapie die gericht is op de hypoxische cellen in een tumor. We hebben enige voortgang geboekt met de ontwikkeing van een induceerbaar xenograft tumor model systeem warin de verschillende hypoxia respons mechanismen uitgeschakeld kunnen worden. Momenteel zijn er muizen studies aan de gang waarin de relevantie van deze mechanismen getest wordt. Ondanks dat er nog veel vragen onbeantwoord blijven is de ontwikkeling van nieuwe therapeutische middelen door de pharmaceutische industrie in volle gang. Het succes van nieuwe therapeutische middelen zal afhangen van een rationele en zorgvuldige invoering in de kliniek. Vanwege de zeer heterogene aard van tumor hypoxia zal het aan te raden zijn om een dergelijke nieuwe therapie alleen te gebruiken in combinatie met traditionele behandelswijzen.

\section{Referenties}

1. Pettersen EO, Juul NO, Ronning OW: Regulation of protein metabolism of human cells during and after acute hypoxia, Cancer Res 1986, 46:4346-4351

2. Kraggerud SM, Sandvik JA, Pettersen EO: Regulation of protein synthesis in human cells exposed to extreme hypoxia, Anticancer Res 1995, 15:683-686

3. Koumenis $C$, Naczki C, Koritzinsky $M$, Rastani S, Diehl A, Sonenberg $N$, Koromilas A, Wouters BG: Regulation of protein synthesis by hypoxia via activation of the endoplasmic reticulum kinase PERK and phosphorylation of the translation initiation factor eIF2alpha, Mol Cell Biol 2002, 22:7405-7416

4. Arsham AM, Howell J1, Simon MC: A Novel Hypoxia-inducible Factorindependent Hypoxic Response Regulating Mammalian Target of Rapamycin and Its Targets, J. Biol. Chem. 2003, 278:29655-29660

5. Liu L, Cash TP, Jones RG, Keith B, Thompson CB, Simon MC: Hypoxia-induced energy stress regulates mRNA translation and cell growth, Mol Cell 2006, 21:521-531

6. Dostie J, Ferraiuolo $M$, Pause A, Adam SA, Sonenberg $N$ : A novel shuttling protein, $4 \mathrm{E}-\mathrm{T}$, mediates the nuclear import of the mRNA $5^{\prime}$ cap-binding protein, elF4E, Embo ] 2000, 19:3142-3156

7. Andrei $M A$, Ingelfinger $D$, Heintzmann $R$, Achsel $T$, Rivera-Pomar $R$, Luhrmann $R$ : A role for eIF4E and elF4E-transporter in targeting mRNPs to mammalian processing bodies, Rna 2005, 11:717-727

8. Blais JD, Filipenko $V$, Bi M, Harding HP, Ron D, Koumenis C, Wouters BG, Bell JC: Activating transcription factor 4 is translationally regulated by hypoxic stress, Mol Cell Bol 2004, 24:7469-7482

9. Koritzinsky $M$, Seigneuric $R$, Magagnin $M G_{y}$ van den Beucken $T$, Lambin $P$, Wouters BG: The hypoxic proteome is influenced by gene-specific changes in mRNA translation, Radiother Oncol 2005, 76:177-186 
10. Cardenas-Navia LI, Yu D, Braun RD, Brizel DM, Secomb TW, Dewhirst MW: Tumor-dependent Kinetics of Partial Pressure of Oxygen Fluctuations during Air and Oxygen Breathing, Cancer Res 2004, 64:6010-6017

11. Lang KJ, Kappel A, Goodall GJ: Hypoxia-inducible factor-1alpha mRNA contains an internal ribosome entry site that allows efficient translation during normoxia and hypoxia, Mol Biol Cell 2002, 13:1792-1801

12. Stein I, Itin A, Einat P, Skaliter R, Grossman Z, Keshet E: Translation of vascular endothelial growth factor mRNA by internal ribosome entry: implications for translation under hypoxia, Mol Cell Biol 1998, 18:3112-3119

13. Thoma C, Bergamini G, Galy B, Hundsdoerfer P, Hentze MW: Enhancement of IRES-mediated translation of the c-myc and BiP mRNAs by the poly(A) tail is independent of intact eIF4G and PABP, Mol Cell 2004, 15:925-935

14. Suzuki $Y$, Ishihara $D$, Sasaki $M$, Nakagawa $H$, Hata $H$, Tsunoda $T$, Watanabe $M$, Komatsu T, Ota $T$, Isogai $T$, Suyama A, Sugano S: Statistical analysis of the $5^{\prime}$ untranslated region of human mRNA using "Oligo-Capped" cDNA libraries, Genomics 2000, 64:286-297

15. Bhattacharya $S$, Michels $C L$, Leung $M K$, Arany $Z P$, Kung $A L$, Livingston $D M$ : Functional role of p35srj, a novel p300/CBP binding protein, during transactivation by HIF-1, Genes Dev 1999, 13:64-75

16. Yin Z, Haynie J, Yang X, Han B, Kiatchoosakun S, Restivo J, Yuan S, Prabhakar NR, Herrup K, Conlon RA, Hoit BD, Watanabe M, Yang YC: The essential role of Cited2, a negative regulator for HIF-1alpha, in heart development and neurulation, Proc Natl Acad Sci U S A 2002, 99:10488-10493

17. Bartel DP: MicroRNAs: genomics, biogenesis, mechanism, and function, Cell $2004,116: 281-297$

18. Kulshreshtha R, Ferracin M, Wojcik SE, Garzon R, Alder $H$, Agosto-Perez FJ, Davuluri $R$, Liu $C G$, Croce $C M$, Negrini $M$, Calin GA, Ivan $M$ : A microRNA signature of hypoxia, Mol Cell Biol 2007, 27:1859-1867

19. Hebert C, Norris K, Scheper MA, Nikitakis N, Sauk JJ: High mobility group A2 is a target for miRNA-98 in head and neck squamous cell carcinoma, Mol Cancer $2007,6: 5$ 


\section{Acknowledgments/ Dankwoord}

First of all I would like to thank my promoters Brad Wouters and Philippe Lambin. Dear Brad, with you as my daily supervisor I learned so many things over the last 5 years. I lost count of all those times that you entered the lab with a new, exciting idea that we should test out immediately. You were always very inspiring and enthusiastic. Chantal and I are very grateful for the opportunity that you have given us to join your new lab in Toronto.

Dear Philippe, I believe that you are the vital link between clinic and laboratory. You certainly succeeded in making MaastRo widely know. Thanks for giving me the opportunity to be part of that.

Dear Marianne, thanks for all your help and support during my PhD period. You were always very involved and interested in all ongoing projects. Also you were always amongst the first to give helpful comments and suggestions for my papers and grant applications. Thanks for that! I'm really glad that we continue to work together in the future!

Beste Kim, wat had ik zonder jou moeten beginnen? Je was er bijna vanaf het begin bij. Ik wil je heel erg bedanken voor al het werk dat je voor mijn boekje hebt verricht. Hoe je het beste een gel kunt laden hebben we geloof ik nooit opgelost of wel? Misschien komt dat er ooit nog van. Ik ben blij dat je nu een van mijn paranimfen bent. Ik wil jou en Pascal heel veel geluk toe wensen met de kleine die op komst is!

Best Barry, we kennen elkaar nu een hele tijd. Uitgezonderd van mijn kleine trip naar Luik hebben we altijd samen gewerkt. Ik denk nog vaak terug aan die toffe tijd bij pathologie. Natuurlijk ben je veel meer dan alleen maar een collega! Vele uurtjes hebben we doorgebracht met fitnessen, katannen en de beruchte LAN-parties. Helaas staat dat allemaal 
even stil nu Chantal en ik in Toronto zitten. Ik wil je bedanken dat je paranimf van mij wil zijn! Verder wil ik je heel veel succes wensen met je ambitieuze plan om klinisch chemicus te worden!

Beste Michael, we zijn bijna tegelijkertijd begonnen met ons promotie onderzoek en ronden het nu ook samen af. We hebben heel veel mee gemaakt, denk maar eens aan onze reisjes naar Kopenhagen, Sicilie en Turkije. Bedankt dat je zo'n toffe college was! Ik vind het nog altijd jammer dat we nooit samen naar een groot congres zijn geweest! Ik weet inmiddels dat je het prima naar je zin hebt met je nieuwe baan. Heel veel succes in je verdere carriere! En natuurlijk ook veel succes met het bouwen van je eigen huis.

Beste Kasper, het is alweer bijna 3 jaar geleden dat jij ons 'hypoxia' team kwam versterken. Jouw motivatie en enthousiasme deed ons groepje goed. Bedankt voor al je hulp met mijn onderzoek!

Ik wil alle collega-AlO's Ludwig, Maud, Younan, Asferd, Hugo en Sherry bedanken voor de leuke tijd in het lab! Veel success met het afronden van jullie promotie onderzoek. Verder dank aan alle andere Maastro (ex)collega's Natasja, Jan, Kim P, Renaud en Mieke. Carla, hartstikke bedankt voor alle administratieve ondersteuning!

De "polycomb people"; Willem, Hanneke, Frank en Peggy bedankt voor jullie input tijdens lab en project meetings. En jij Hanneke, als ChIP expert, natuurlijk extra voor je bijdrage aan het CA9 verhaal. Ramon en Jos van pulmonologie jullie ook bedankt voor jullie bijdrage tijdens lab meetings.

Vrienden van het kook clubje, Martijn, Steven en Dennis. Onze donderdag avond was altijd enerverend en natuurlijk het wijn proef weekend in Frankrijk en de talloze stap avonden niet te vergeten! Bedankt voor al die 
leuke momenten! Martijn en Dennis, succes met jullie opleiding tot internist. Steven en Judith veel succes met jullie post-doc in "The Big Apple".

Alle vrienden van het ruitersport centrum "de Groene Vallei", in het speciaal May, Tony en Roy, bedankt voor alle leuke uurtjes op de manege!

Ton en Annelies bedankt voor jullie steun, interesse en natuurlijk al die keren dat wij bij jullie hebben mogen eten als we weer eens lat van het werk terug kwamen. Harry, Wendy en Meggy bedankt voor alle leuke en ontspannende momenten met jullie! Hopelijk snappen jullie nu beter wat ik in dat laboratorium uitvoer. Verder alle vrienden, kennissen en familie bedankt voor jullie belangstelling de afgelopen jaren!

Lieve Mam en Peter bedankt voor al jullie onvoorwaardelijke steun! Jullie staan echt altijd voor ons klaar. Lieve pap, zonder jou zou dit nooit mogelijk zijn geweest!

Lieve Chantal, bedankt dat je er altijd voor me bent en dat we zoveel met elkaar kunnen delen! Samen gaan we een mooie toekomst tegemoet en volgend jaar ben jij aan de beurt! 


\section{Curriculum Vitae}

Twan van den Beucken was born on March $17^{\text {th }}, 1977$ in Venlo, The Netherlands. In 1994 he started the study "Technical Microbiology" at the school of higher education in Venlo, where he in 1998 graduated "cum laude". In the period of 1998 to 2003 Twan worked for biotechnology companies Target Quest and Dyax as associate scientist and senior developmental scientist respectively. Twan started his PhD in July 2003 at the department of radiation oncology (Maastro) within the University of Maastricht, to determine the effect of hypoxia on translational control, gene expression and cell survival in cancer. Twan is currently appointed as a post-doctoral fellow at the department of radiation oncology in Maastricht. As part of this appointment Twan is now working at the Princess Margaret Hospital/ Ontario cancer institute in Toronto, Ontario, Canada. 


\section{List of publications}

Twan van den Beucken, Marianne Koritzinsky, Hanneke Niessen, Ludwig Dubois, Kim Savelkouls, Hilda Mujcic, Barry Jutten, Juraj Kopacek, Sylvia Pastorekova, Philippe Lambin, Willem Voncken, Kasper M.A. Rouschop and Bradly $G$. Wouters. Hypoxia-induced carbonic anhydrase 9 expression is dependent on the unfolded protein response. Submitted

Twan van den Beucken, Michael G. Magagnin, Barry Jutten, Renaud Seigneuric, Philippe Lambin, Marianne Koritzinsky and Bradly G. Wouters. Regulation of mRNA translation is a major contributor to hypoxia induced gene expression. Submitted

Kasper M.A. Rouschop, Twan van den Beucken, Ludwig Dubois, Hanneke Niessen, Kim Savelkouls, Jan Bussink, Hilda Mujcic, Philippe Lambin, Albert J. van der Kogel, Marianne Koritzinsky and Bradly G. Wouters. The unfolded protein response elicits a transcriptional program that augments autophagy upon exposure to hypoxia. Submitted

Femke Hillen, Twan van den Beucken, Petra Hautvast, Bradly G. Wouters, Arjan W, Griffioen. Involvement of hypoxia and HIF-1 $\alpha$ in tumor cell plasticity. In preparation

Magagnin $M G$, van den Beucken $T$, Sergeant $K$, Lambin $P$, Koritzinsky $M$, Devreese B, Wouters BG. The mTOR target 4E-BP1 contributes to differential protein expression during normoxia and hypoxia through changes in mRNA translation efficiency. Proteomics. 2008 Mar;8(5):101928.

Swennen EL, Dagnelie PC, van den Beucken T, Bast A. Radioprotective effects of ATP in human blood ex vivo. Biochem Biophys Res Commun. 2008 Mar 7;367(2):383-7.

Magagnin MG, Sergeant $K$, van den Beucken $T$, Rouschop KM, Jutten $B$, Seigneuric R, Lambin P, Devreese B, Koritzinsky M, Wouters BG. Proteomic analysis of gene expression following hypoxia and reoxygenation reveals proteins involved in the recovery from endoplasmic reticulum and oxidative stress. Radiother Oncol. 2007 Jun;83(3):340-5.

Koritzinsky $M$, Rouschop KM, van den Beucken T, Magagnin MG, Savelkouls K, Lambin P, Wouters BG. Phosphorylation of eIF2alpha is required for mRNA translation inhibition and survival during moderate hypoxia. Radiother Oncol. 2007 Jun;83(3):353-61.

van den Beucken $T$, Magagnin $M G$, Savelkouls $K$, Lambin $P$, Koritzinsky $M$, Wouters BG. Regulation of Cited2 expression provides a functional link 
between translational and transcriptional responses during hypoxia. Radiother Oncol. 2007 Jun; 83(3):346-52.

Twan van den Beucken, Marianne Koritzinsky and Bradly G. Wouters, Translational control of gene expression during hypoxia. Cancer Biology \& Therapy 5:7:749-55, July 2006.

Koritzinsky $M$, Magagnin MG, van den Beucken $T$, Seigneuric $R$, Savelkouls K, Dostie J, Pyronnet S, Kaufman RJ, Weppler SA, Voncken JW, Lambin P, Koumenis C, Sonenberg N, Wouters BG. Gene expression during acute and prolonged hypoxia is regulated by distinct mechanisms of translational control. EMBO J. 2006 Feb 9;

Koritzinsky $M$, Seigneuric $R$, Magagnin MG, van den Beucken $T$, Lambin $P$, Wouters $B G$. The hypoxic proteome is influenced by gene-specific changes in mRNA translation. Radiother Oncol. 2005 Aug; 76(2):177-86.

Wouters BG, van den Beucken T, Magagnin MG, Koritzinsky M, Fels D, Koumenis $C$. Control of the hypoxic response through regulation of mRNA translation. Semin Cell Dev Biol. 2005 Aug-Oct; 16(4-5):487-501.

Blaise $L$, Wehnert $A$, Steukers MP, van den Beucken $T$, Hoogenboom HR, Hufton SE. Construction and diversification of yeast cell surface displayed libraries by yeast mating: application to the affinity maturation of Fab antibody fragments. Gene. 2004 Nov 24;342(2):211-218.

Wouters BG, van den Beucken T, Magagnin MG, Lambin P, Koumenis C. Targeting hypoxia tolerance in cancer. Drug Resist Updat. 2004 Feb;7(1):25-40.

van den Beucken $T$, Pieters $H$, Steukers $M$, van der Vaart $M$, Ladner $R C$, Hoogenboom HR, Hufton SE. Affinity maturation of Fab antibody fragments by fluorescent-activated cell sorting of yeast-displayed libraries. FEBS Lett. 2003 Jul 10;546(2-3):288-94.

van den Beucken T, van Neer N, Sablon E, Desmet J, Celis L, Hoogenboom HR, Hufton SE. Building novel binding ligands to B7.1 and B7.2 based on human antibody single variable light chain domains. J Mol Biol. $2001 \mathrm{Jul}$ $13 ; 310(3): 591-601$.

Hufton SE, van Neer N, van den Beuken T, Desmet J, Sablon E, Hoogenboom HR.

Development and application of cytotoxic T lymphocyte-associated antigen 4 as a protein scaffold for the generation of novel binding ligands. FEBS Lett. 2000 Jun 23;475(3):225-31. 